\title{
ALBERTA VEGETATION SPECIES LIST AND SPECIES GROUP CHECKLISTS
}


Digitized by the Internet Archive in 2015 


\section{ALBERTA VEGETATION SPECIES LIST AND SPECIES GROUP CHECKLISTS}

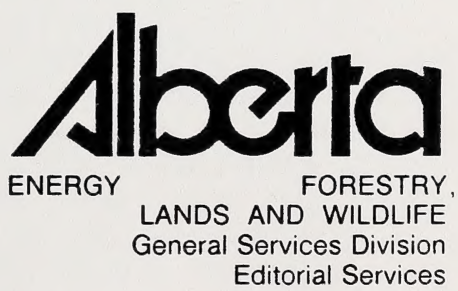


Pub. No.: Ref. 56

ISBN : 0-86499-778-7

For copies of this report, contact:

Editorial Services Unit General Services Division

Energy/Forestry, Lands and Wildlife

6 th Floor, South Petroleum Plaza,

9915 - 108 Street

Edmonton, Alberta T5K 2C9

Telephone: (403) 422-0858 


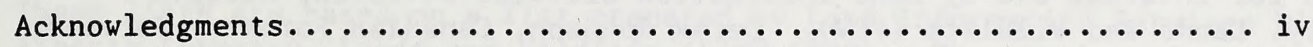

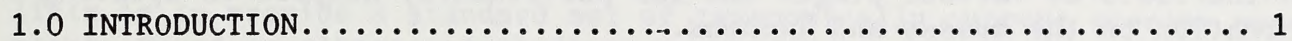

1.1 Components of Alberta Vegetation Species List..............

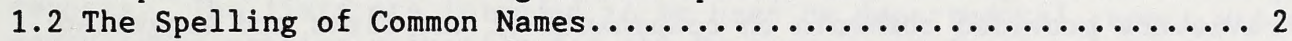

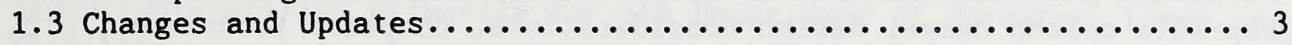

2.0 REFERENCES....................................... 5

ALBERTA VEGETATION SPECIES LIST...................... 1-69

ALBERTA TREE SPECIES CHECKLIST $\ldots \ldots \ldots \ldots \ldots \ldots \ldots \ldots \ldots \ldots \ldots \ldots \ldots$

ALBERTA SHRUB SPECIES CHECKLIST....................... 1-3

ALBERTA FORB SPECIES CHECKLIST ....................... 1-23

ALBERTA GRASS SPECIES CHECKLIST $\ldots \ldots \ldots \ldots \ldots \ldots \ldots \ldots \ldots \ldots \ldots \ldots$

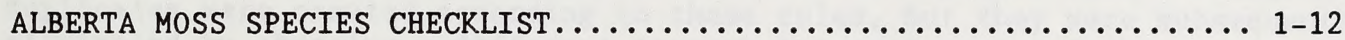

ALBERTA LICHEN SPECIES CHECKLIST....................... 1-11 


\section{ACKNOWLEDGMENTS}

Many people helped with the production of these lists. Louise Horstman, Pecan Resources, prepared the initial review and update of a species list originally printed for internal use by Land Information Services Division, Alberta Forestry, Lands and Wildlife (FLW). Derek Johnson, Canadian Forestry Service, reviewed the lists at various stages and was particularly helpful in updating the information on the mosses and lichens.

Several reviewers provided comments on components of the lists and suggested more appropriate common names, design considerations, computer techniques, etc. We would like to thank the following for their efforts: Natural and Protected Areas Program, Public Lands Division, FLW -- Lorna Allen, Joyce Gould, John Rintoul; Provincial Museum of Alberta -- Julie Hrapko; University of Alberta, Dept. of Botany -- Dale Vitt; Alberta Forest Service -- Brian Maier; University of Calgary, Department of Biological Sciences -- Bonnie Smith; Charles Bird.

The input of species names into the data base was largely completed by Brad Thomas. The coordination and editing of the lists was the responsibility of David M. Ealey of the Editorial Services Unit, General Services Division, Alberta Energy/Forestry, Lands and Wildlife. 


\subsection{INTRODUCTION}

These lists are based on a list prepared by Land Information Services Division, Alberta Forestry, Lands and Vildlife (LISD 1988). The purpose of producing such lists is to provide a standard set of taxonomically current species names for plants found in Alberta, including accepted common names for use in departmental reports. The lists are intended to be used by departmental report writers and consultants writing primarily for the Department of Forestry, Lands and Wildlife.

Plant species names have been brought up-to-date with current taxonomy and common usage, according to the criteria stated below. These names have been entered into a dBASE IV data base, to allow future revisions of the plant names. The master list is maintained by Editorial Services, so that revisions can be prepared consistently. Any changes or additions should be forwarded to the unit as outlined in Section 1.3.

\subsection{Components of Alberta Vegetation Species List}

The following identifies each of the eight columns that make up the species list: 1. CODE - A seven-letter code consisting of the first four letters of the genus name plus the first three letters of the species name. Occasionally duplicates were created according to these rules, but they were subsequently eliminated by choosing a different seventh letter for one of the species. Modifications were made for species having three-letter genus names by adding a fourth letter from the species portion of the Latin name. The computer code is the index for identification and sorting of each-vegetation species and is thus in the first column of the vegetation species list. In addition, the code will be useful as a short-form reference for workers generating species lists for projects or study areas; this is why the code is placed in the last column before the checkpoint in the species group checklists.

2. LATIN NAME - Primary sources of Latin names were as follows for different species groups: vascular plants, Moss (1983); mosses, Ireland et al. (1987); liverworts and hornworts, Stotler and Crandall-Stotler (1977); lichens, Egan (1987). Other sources were used to fill gaps and to add more recent nomenclature (see 2.0 References).

3. AUTHORITY - Sources of authorities for Latin names were as above. In the vegetation species list, the taxonomic authorities are combined with the Latin name, in a single column and separated by a dash.

4. ENGLISH COMMON NAME, adjectival portion - The English common name was separated into an adjectival first part and a root name (next column). This was to facilitate computer sorting on the first part of the name and also sorting of similar types of plants. Where the common name consisted of only one word, that name was usually listed in this first column, unless the name was clearly a root word.

5. ENGLISH COMMON NAME, root word or genus - The second part of the English common name is frequently a name for the genus. When they are, these portions of the name are incorporated in this second of two columns.

6. SOURCE - This refers to the source for the English common name and 
is largely used for vascular plants. E.H. Moss (EHM) was the primary source of these common names, and it was Moss's first-listed common name for a species that was selected. Where Moss did not show a common name, A.C. Budd (ACB), who was the initiator of Budd's Flora of the Canadian Prairie Provinces (Looman and Best 1987), was the source. If neither source had a common name, then a genus' common name might be used; however, no source was listed for such names.

occasionally, the common name for an earlier synonym from Budd was used if Moss had no common names. In their review comments, botanists strongly favoured some names by Budd over Moss' names, and as a result the (ACB) name was used. The source (BOT) refers to botanists in Alberta who have expressed a concerted disapproval of Moss's or Budd's names or have anglicized the Latin names directly or else have developed names, where neither Moss nor Budd have provided names. A few other sources were identified for plants new to the province or for plants not named by Moss or Budd or for nonvascular plants. These sources were as follows: (BCM) - B.C. Ministry of Forests reports; (DJ1) - Johnson (1987); (ECW) - Alex et al. (1980); (G\&I) - Glime and Iwatsuki (1990); (GLI) Glime (1989); (H\&C) - Hitchock et al. (1950-1969); (P\&C) - Porsild and Cody (1979); and (W\&W) - Wallis et al. (1987).

7. FAMILY - The Latin family name is taken from the scientific sources identified above.

8. F (form) - The form or plant type is indicated in this column: A tree; B - shrub; C - forb; D - mosses and liverworts; G - graminoid; L - lichen. Generally, the way in which these forms are applied is traditional; however, determination of what constitutes a shrub was difficult, given the range of definitions found. The definition chosen was to reflect similar usage in Ontario (Soper and Heimburger 1982). A shrub was defined as a low-growing, perennial plant, usually with several main stems, arising near the ground, that are at least partially woody.

Species group checklists are included which group all taxa belonging to the distinct forms identified above. The checklists do not include the authority or source information and are rearranged to be more useful for project use. The number of taxa (nearly all are species, except for a few distinct hybrids) included in each checklist are as follows: trees - 28; shrubs - 160; forbs 1208; grasses - 361; mosses - 601; lichens - 578. Total vascular taxa amount to 1757 species, while lichens and mosses combine to 1179 species. The overall total of vegetation taxa for Alberta is 2936 species.

\subsection{The Spelling of Common Names}

Although the sources identified above were used to determine the accepted common name, they produced such a variance in word formation/spelling that the need was obvious for a standard approach to spelling of common names. The rules outlined in Loomis and Best (1987) and in Alex et al. (1980) were used because of their consistency.

These rules are as follows:

1. Written as one word

(a) When the modified word is plant or a type of plant, as in leadplant, skunkbush, peatree, peavine, jewelweed, lungwort; except when the modifier is more than one word, as in Kentucky coffee tree, or a hyphened word, as in silk-tassel bush, or a proper noun, as in Virginia creeper.

(b) When the modified word is a part of a plant, as in juneberry, 
twayblade, buffalobur, coneflower, leatherleaf, bladderpod, balsamroot, bugseed, twistedstalk, bluestem, buckthorn, wormwood.

(c) When the modified word is a part of animal anatomy, as in arrowhead, bluelips, cattail, beardtongue; except when the modifier is in the possessive case, as in bird's-eye, crane's-bill.

(d) When the words are figurative or suggestive, as in beggarticks, fairybells, meadowsweet, paintbrush, except when the modifier is in the possessive case, as in baby's-breath, or when it is a proper noun or the adjectival form of a proper noun, as in Venus-slipper, Indian-pipe, or when letters demand separation for ease in reading or pronunciation, as in morning-glory.

\section{Written as separate words}

a) When the modified word is taxonomically correct, as in red clover (genus Trifolium), alkali grass (family Gramineae), fringed milkwort (genus Polygala), woolly plantain (genus Plantago).

(b) When the modifier is the word common, false, mock, wild as in common camas, false flax, mock pennyroyal, wild chives.

(c) When the modifier is a proper noun, as in Douglas hawthorn, Mackenzie's hedysarum.

(d) Exceptions in 1 .

3. Written hyphened

(a) When the modified word is not taxonomically correct, as in sweet-clover (genus Melilotus, not genus Trifolium), whitlow-grass (genus Draba, not family Gramineae), sea-milkwort (genus Glaux, not genus Polygala), water-plantain (genus Alisma, not genus Plantago), except in a few instances of spelling of long standing, as in burdock, buckwheat.

(b) When the modifier is a compound, as in round-leaved hawthorn, and whether or not the modified word is taxonomically correct, as in salt-meadow grass (family Gramineae), blue-eyed grass (genus Sisyrinchium, not family Gramineae).

(c) In certain three- or four-word groups, as balm-of-Gilead, but ter-and-eggs, grass-of-Parnassus, lily-of-the-valley, touch-me-not .

(d) Exceptions in 1 .

\subsection{Changes and Updates}

Information on any new species of plants discovered for Alberta, or taxonomic revisions, will be incorporated into the master species list where appropriate. When sufficient changes are accumulated to warrant reprinting, new lists will be generated. All users are encouraged to forward any changes/errors that are discovered.

To make changes more easily and accurately, the following form should be filled out and sent to this address: Editorial Services Unit, General Services Division, Alberta Energy/Forestry, Lands and Wildlife, 6 th Floor, South Tower Petroleum Plaza, 9915 - 108 St., Edmonton, Alberta T5K 2C9. 
VEGETATION SPECIES CHANGES/REVISIONS:

For each species, please submit the following information.

Species Name

Taxonomic Authority

Common Name

Family

Plant Type (spell out)

Reference

Collector (if known)

Approximate Location (for new species)

Proposed Change

Why? 


\subsection{REFERENCES}

Alex, J.F., R. Cayouette and G. A. Mulligan. 1980. Common and botanical names of weeds in Canada. Expert Committee on Weeds, Research Branch, Agriculture Canada, Ottawa. 132 pp.

Bird, C.D. 1971. Plants of the alpine and upper subalpine parts of Ram Mountain. Department of Biology, University of Calgary, Calgary, Alberta. Mimeogr. 5 pp.

Bird, C.D. 1972. A catalogue of the lichens reported from Alberta, Saskatchewan and Manitoba. Department of Biology, University of Calgary, Calgary, Alberta. Mimeogr. 49 pp.

Bird, C.D. 1973. Species collected in Alberta on the first 1971 foray of the American Bryological and Lichenological Society. Part 1. Introduction and lichens. The Bryologist 76:388-402.

Bird, C.D. 1973. A new catalogue of the bryophytes reported from Alberta, Saskatchewan and Manitoba. Dept. of Biology, Univ. of Calgary, Calgary. Mimeogr. 63 pp.

Bird, C.D., and W.S. Hong. 1975. The hepatic flora of Alberta: a phytogeographical analysis. Can. J. Bot. 53:1745-1768.

Bird, C.D. 1978. Keys to the lichens of west-central Canada (revised). Department of Biology, University of Calgary, Calgary, Alberta. Mimeogr. $171 \mathrm{pp}$.

Brodo, I.M., and D.L. Hawksworth. 1977. Alectoria and allied genera in North America. Opera Botanica 42:1-164.

Case, J.W. 1977. Lichens on Populus tremuloides in western central Alberta, Canada. The Bryologist $80(1): 48-70$.

Douglas, G.W., W.L. Peterson and A.C. Skorepa. 1981. A preliminary checklist of the mosses and lichens in the Fort McMurray area, Alberta, Canada. Can. J. Bot. 59:1456-1464.

Egan, R.S. 1987. A fifth checklist of the lichen-forming, lichenicolous and allied fungi of the continental United States and Canada. The Bryologist $90(2): 77-173$.

Egan, R.S. 1989. Changes to the "Fifth checklist of the lichen-forming, lichenicolous and allied fungi of the continental United States and Canada." Edition I. The Bryologist 92(1):68-72.

Egan, R.S. 1990. Changes to the "Fifth checklist of the lichen-forming, lichenicolous and allied fungi of the continental United States and Canada." Edition II. The Bryologist 93(2):211-219.

Glime, J.M. 1989. Should mosses have common names? Evansia 6(1):1-6. 
Glime, J.M., and Z. Iwatsuki. 1990. Should mosses have common names? Part 3. The common names of the primitive orders. Evansia 7(1):9-11.

Hale, M.E., Jr. 1979. How to know the lichens. Second edition. Wm. C. Brown Co., Dubuque, Iowa. 246 pp.

Hitchcock, C.L., et al. 1950-1969. Vascular plants of the Pacific Northwest. Vols. 1-5. University of Washington Press, Seattle.

Holland, W.D., and G.M. Coen. (eds). 1982. Ecological (biophysical) land classification of Banff and Jasper national parks. Vol. II. Soil and vegetation resources. Alberta Institute of Pedology and Canadian Forestry Service, Edmonton, Alberta.

Ireland, R.R., G.R. Brassard, W.B. Schofield and D.H. Vitt. 1987. Checklist of the mosses of Canada II. Lindbergia 13:1-62.

John, E.A. 1989. The saxicolous lichen flora of Jonas Rockslide, Jasper National Park, Alberta. The Bryologist 92(1):105-111.

Johnson, J.D. 1987. A botanically interesting peatland in north-central Alberta. Alta. Nat. 17(1):1-3.

Johnson, J.D. 1989a. Additions to the lichen flora of Alberta, Saskatchewan, and Manitoba. Can. Field-Nat. 103(1):96-99.

Johnson, J.D. 1989b. Additions to the bryophyte flora of Alberta, Saskatchewan, and Manitoba. Can. Field-Nat. 103(4):497-508.

Johnson, J.D. 1990. Results of the 1989 May Species Count -- plants in flower. Alta. Nat. 20(1):6-13.

Karnefelt, I. 1979. The brown fruticose species of Cetraria. Opera Botanica 46:1-150.

Li, Yenhung, and J.M. Glime. 1989. Should mosses have common names? Part 2. The common names of Sphagnum. Evansia 6(2):25-27.

Land Information Services Division (LISD). 1988. Vegetation species list for Alberta. Unpub. report, prep. by LISD, Alberta Forestry, Lands \& Wildlife.

Looman, J., and K.F. Best. 1987. Budd's flora of the Canadian Prairie Provinces. Research Branch, Agriculture Canada, Publication 1662. 863 pp.

Moss, E.H. 1983. Flora of Alberta. Second Edition, revised by J.G. Packer. University of Toronto Press, Toronto. $687 \mathrm{pp}$.

Pinel, H.W. (ed.). 1981. Calgary's natural areas. Natural Areas Committee, Calgary Field Naturalists' Society, Calgary, Alberta.

Porsild, A.E., and W.J. Cody. 1979. Vascular plants of continental Northwest Territories, Canada. National Museums of Natural Sciences, National 
Museums of Canada. 667 pp.

Skorepa, A.C., and D.H. Vitt. 1976. A quantitative study of epiphytic lichen vegetation in relation to $\mathrm{SO}_{2}$ pollution in western Alberta. Environ. Can., Can. For. Serv., North For. Res. Cent., Edmonton, Alberta. Inf. Rep. NOR-X-161. 26 pp.

Soper, J.H., and M.L. Heimburger. 1982. Shrubs of Ontario. Royal Ontario Museum, Publications in Life Sciences. $495 \mathrm{pp}$.

Stotler, R., and B. Crandall-Stotler. 1977. A checklist of the liverworts and hornworts of North America. The Bryologist 80(3):405-425.

Thomson, J.W. 1979. Lichens of the Alaskan Arctic Slope. University of Toronto Press, Toronto, Ontario. $314 \mathrm{pp}$.

Thomson, J.W. 1984. American Arctic lichens. 1. The Macrolichens. Columbia University Press, New York, New York. 504 pp.

Vitt, D.H. 1977. A checklist of the Hepaticae of Alberta and the Yukon Territory. Dept. of Botany, Univ. of Alberta, Edmonton. Mimeogr. 11 pp.

Vitt, D.H., and D.G. Horton. 1979. Bryophytes new to Alberta, Yukon Territory and Northwest Territories, Canada. The Bryologist 82(3):409-416.

Vitt, D.H., J.E. Marsh and R.B. Bovey. 1988. Mosses, lichens \& ferns of northwest North America. Lone Pine Publishing, Edmonton, Alberta and University of Washington Press, Seattle, Washington. 296 pp.

Wallis, C., C. Bradley, M. Fairbarns and V. Loewen. 1987. The rare flora of Alberta. Volume 3. Species summary sheets. Public Lands Division, Alberta Forestry, Lands and Wildlife.

Wilkinson, K. 1990. A habitat field guide: trees and shrubs of Alberta. Lone Pine Publishing, Edmonton. 191 pp. 



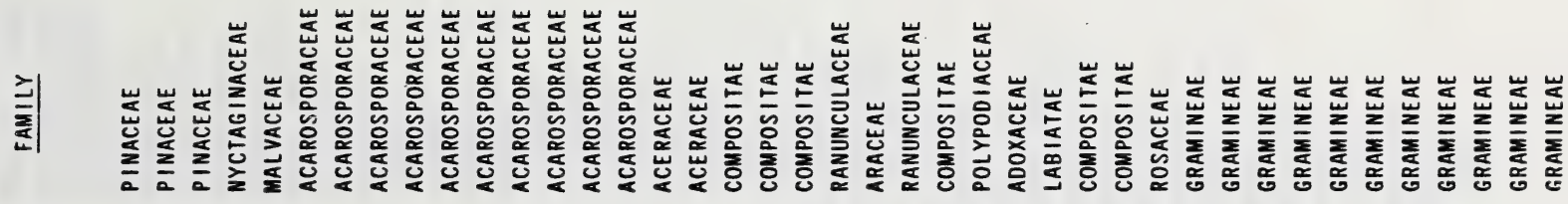

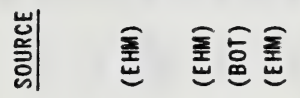

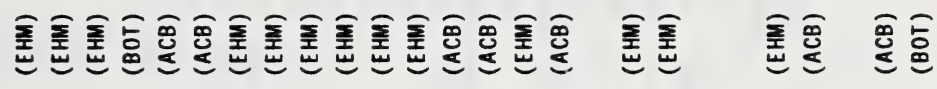

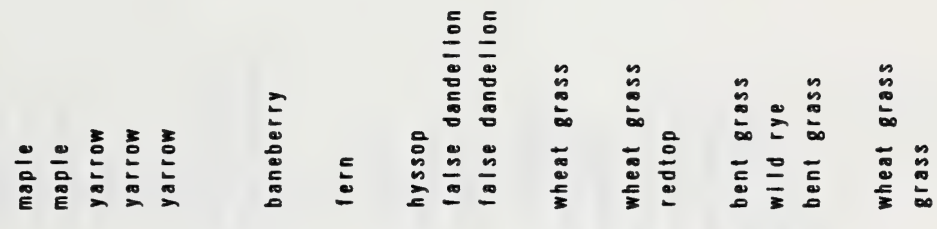

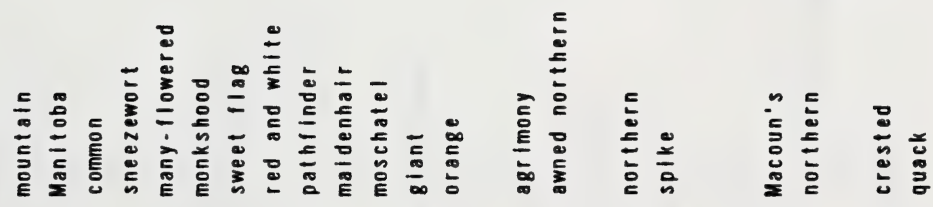

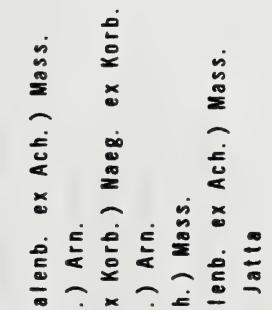

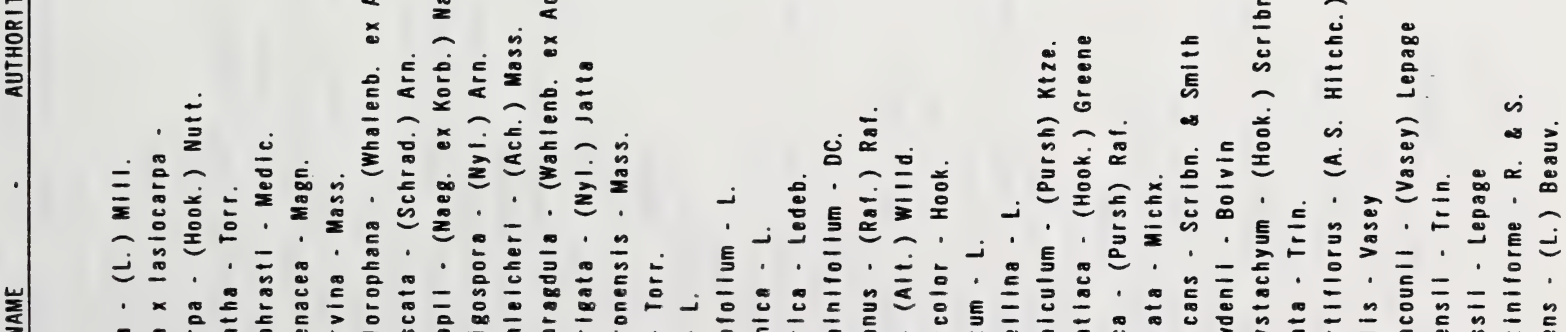

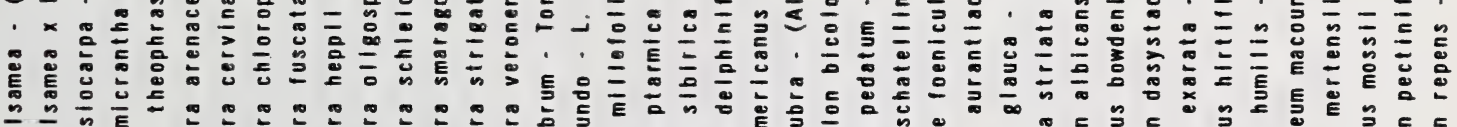

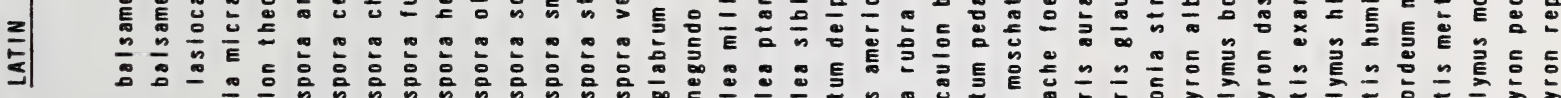

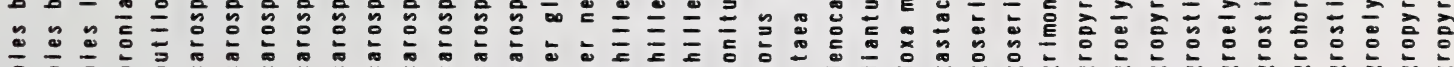

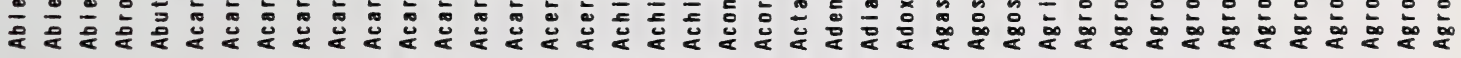

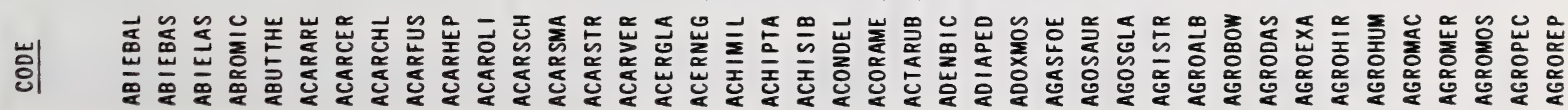




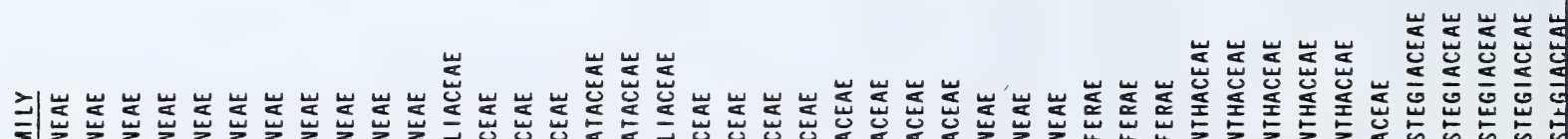

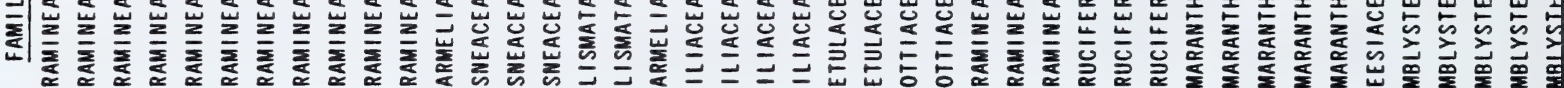

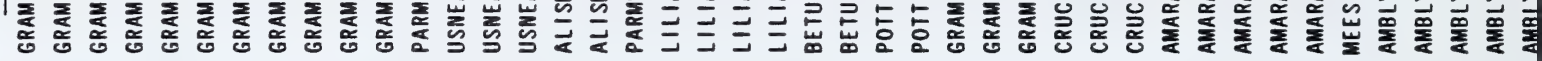

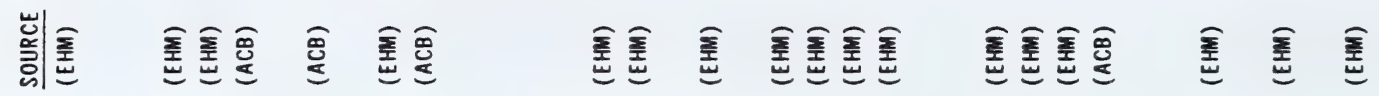

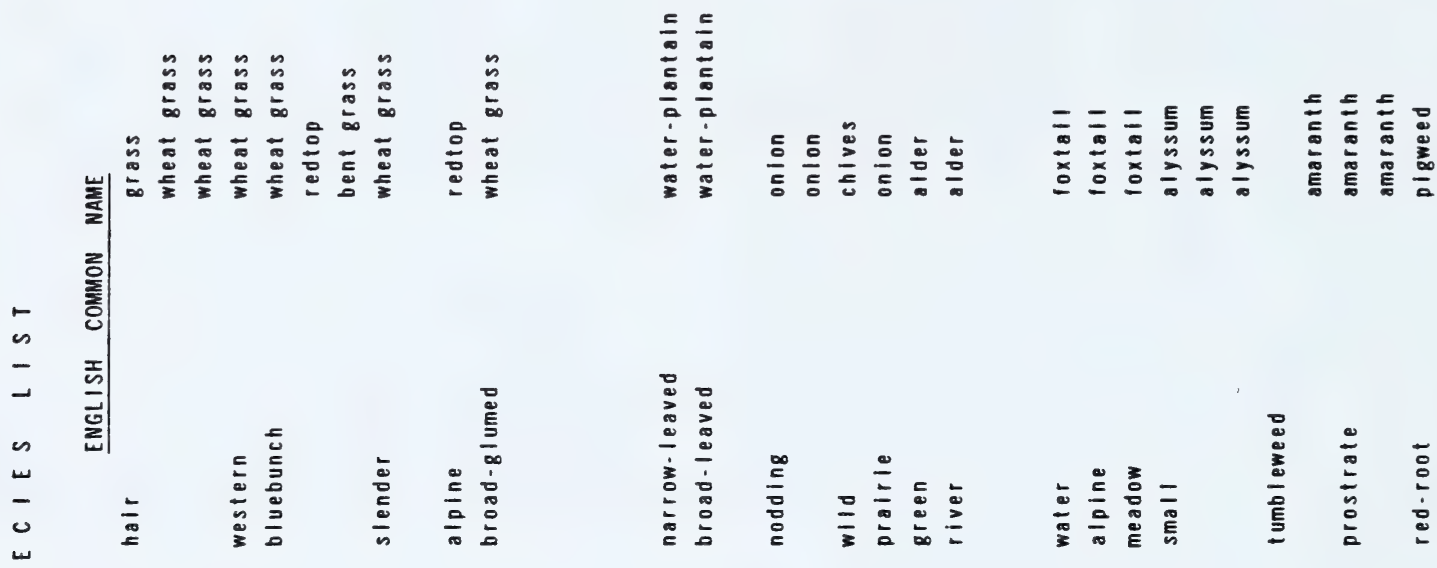

$\square$
$\approx$
$\infty$
$\infty$
$\infty$

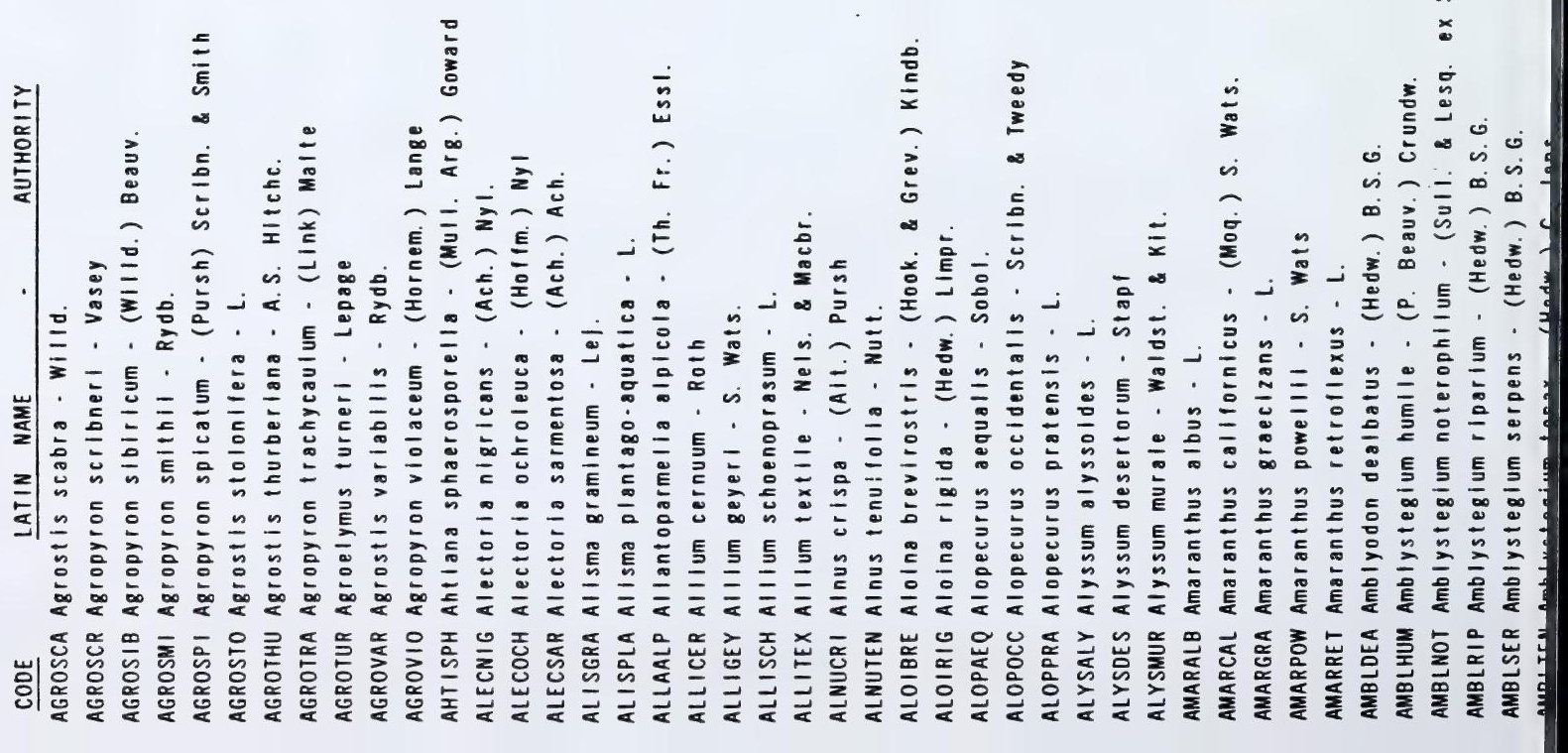



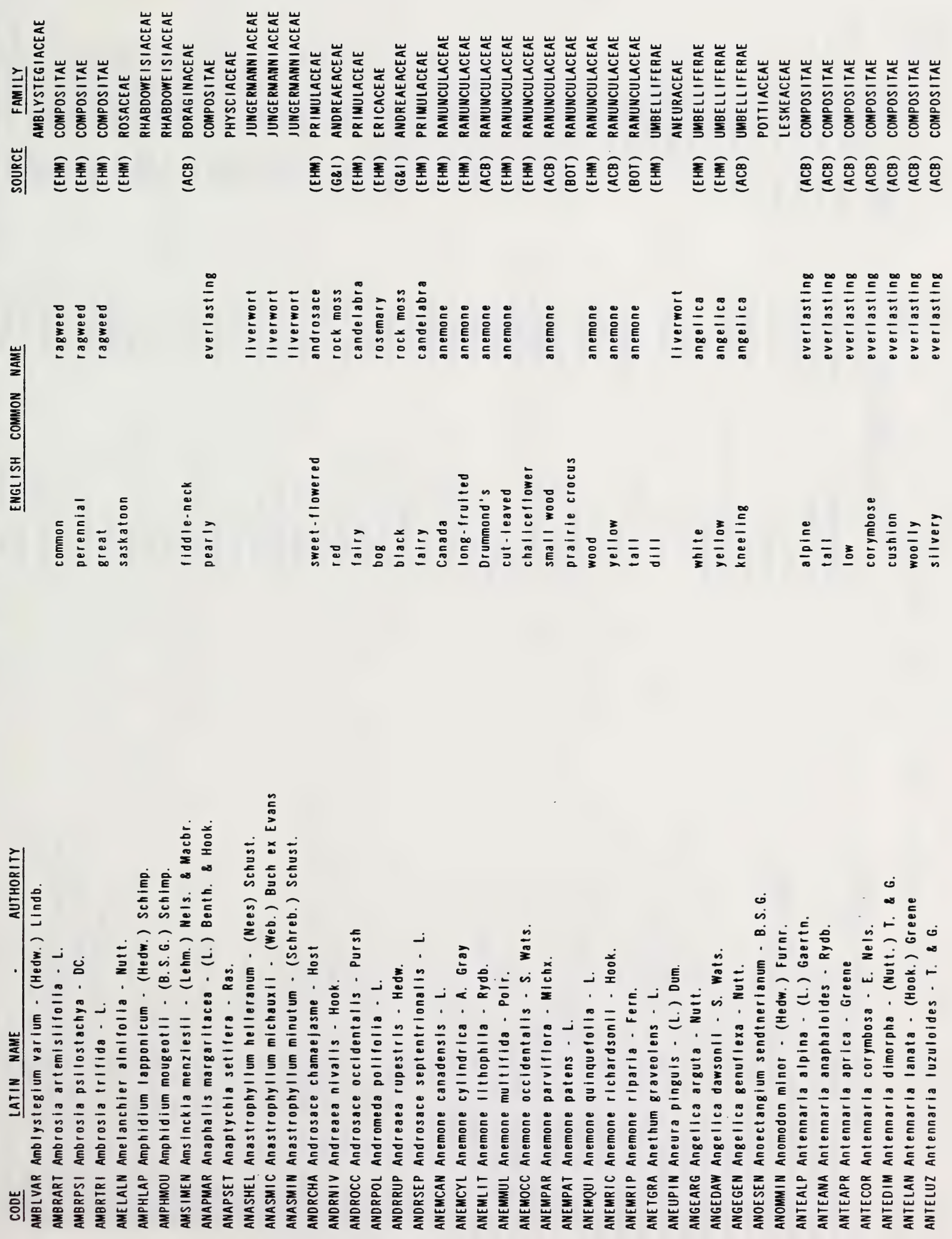


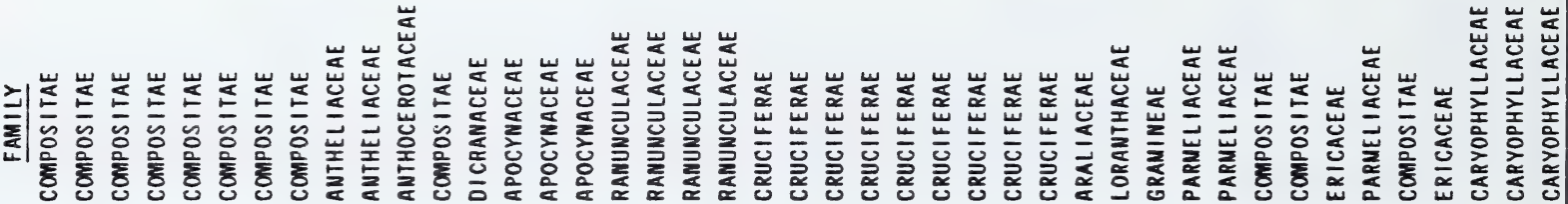

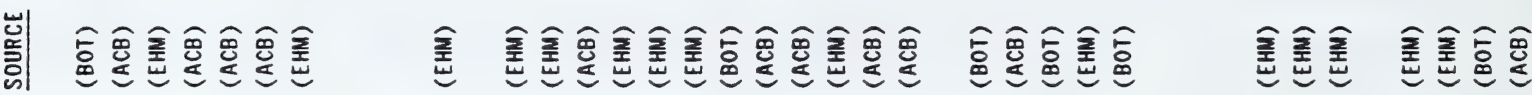

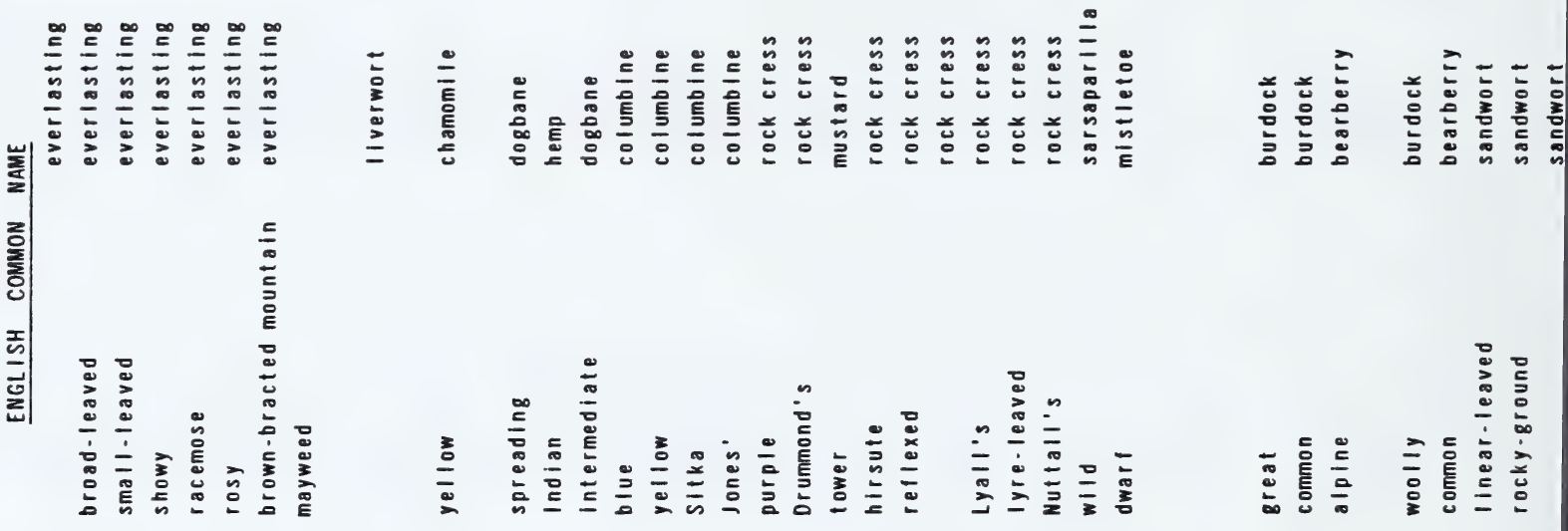

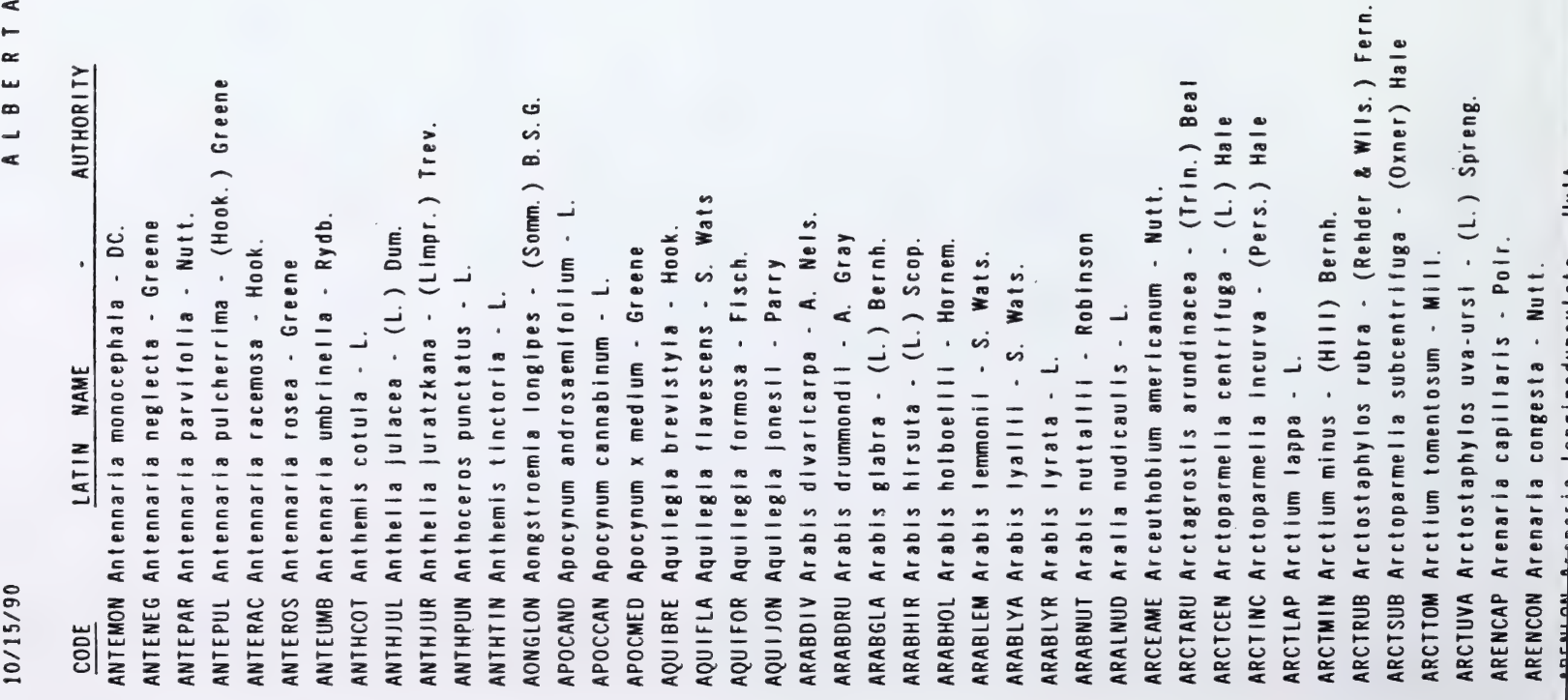




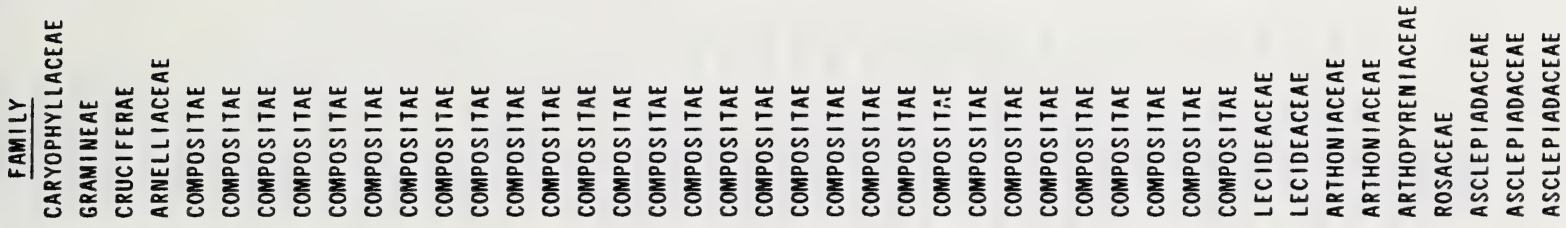

岕岕

这这

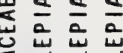

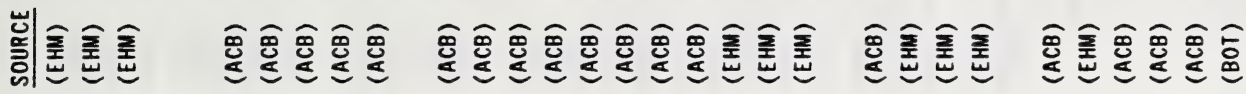

金金金重
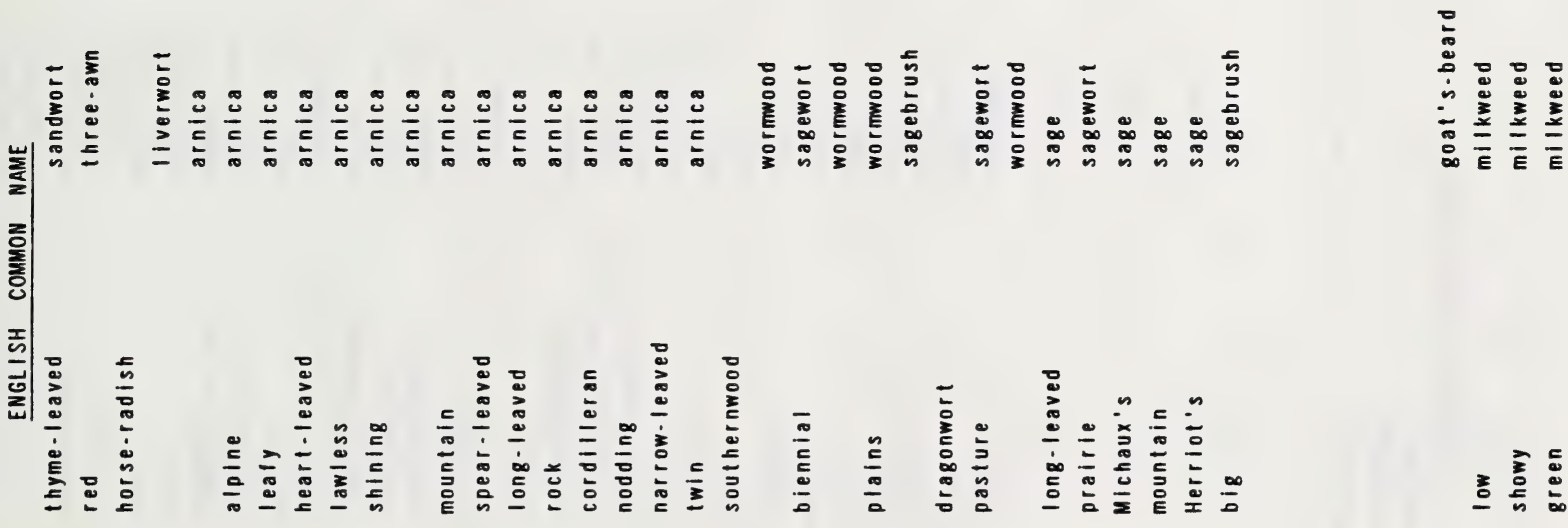

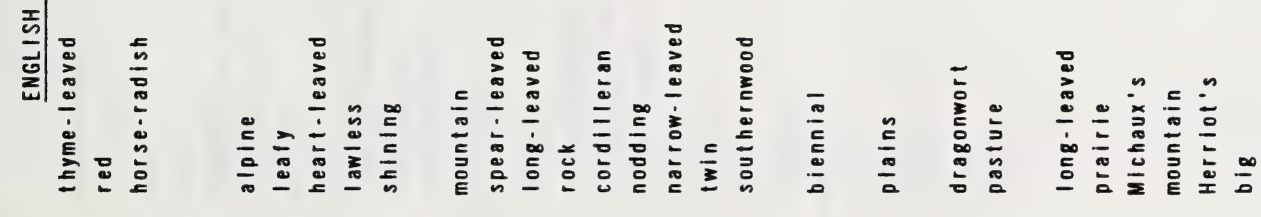

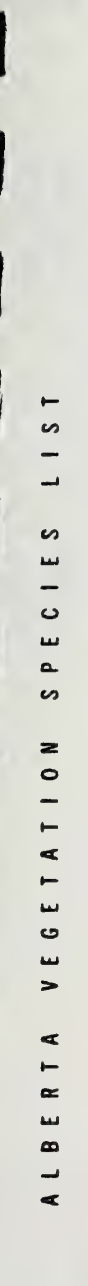

흫

<

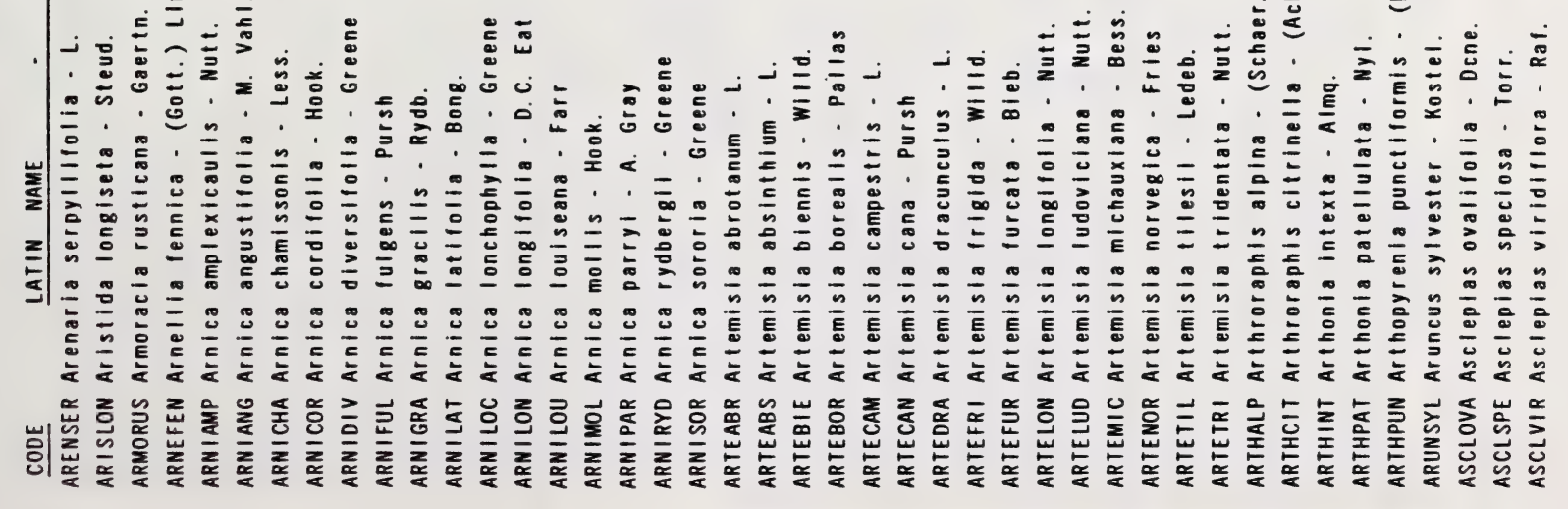

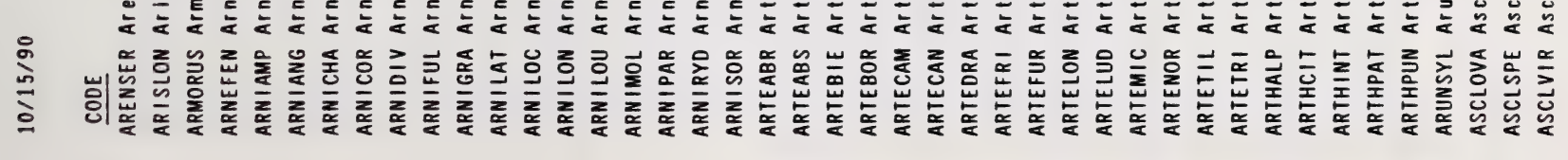




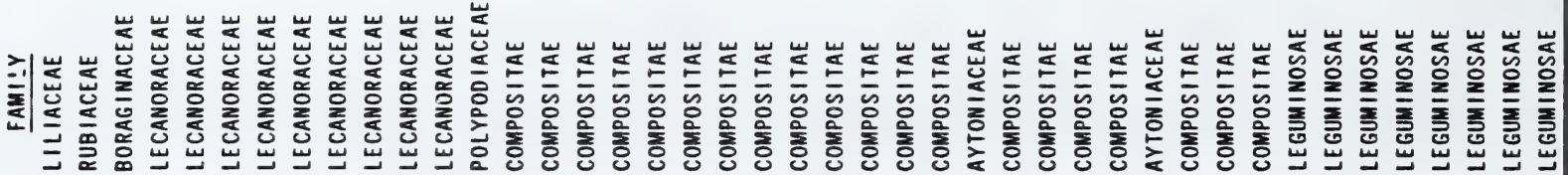

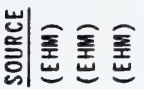
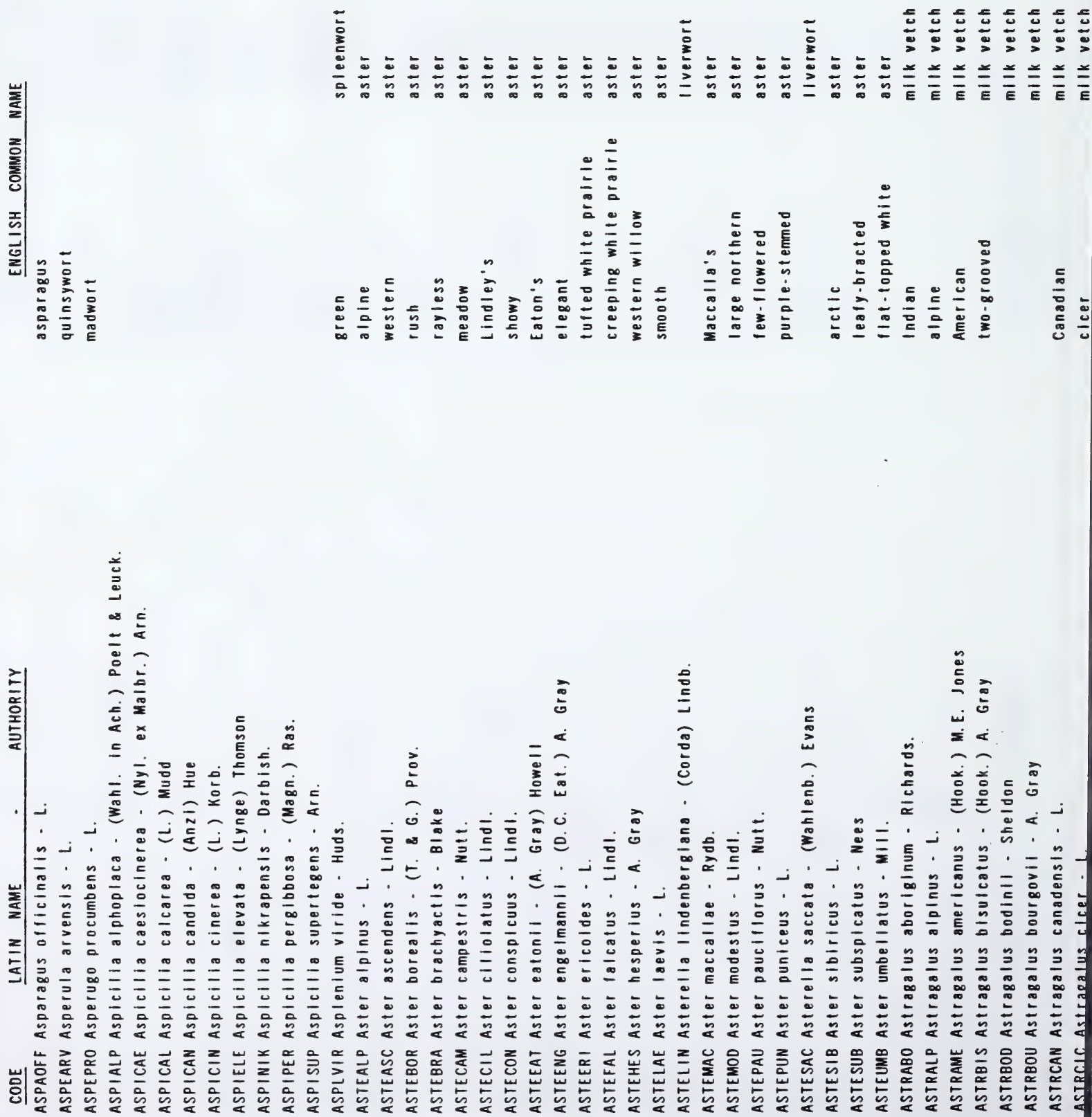


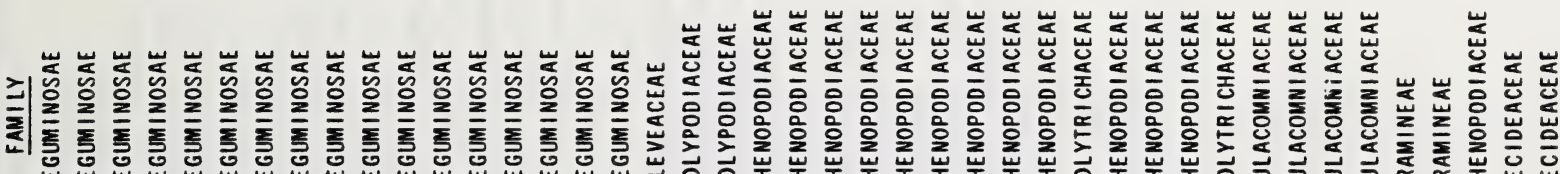

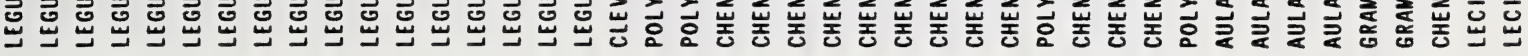

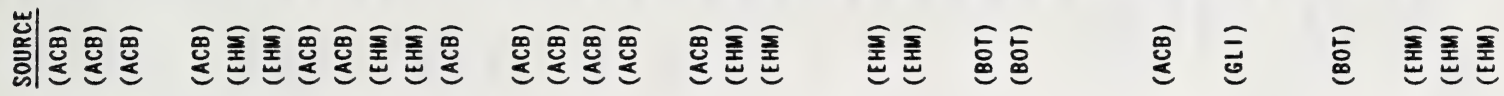

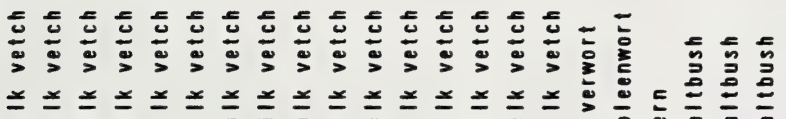

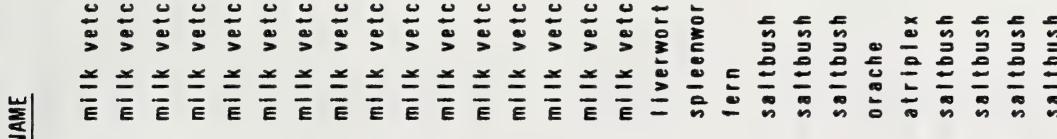

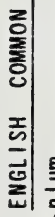

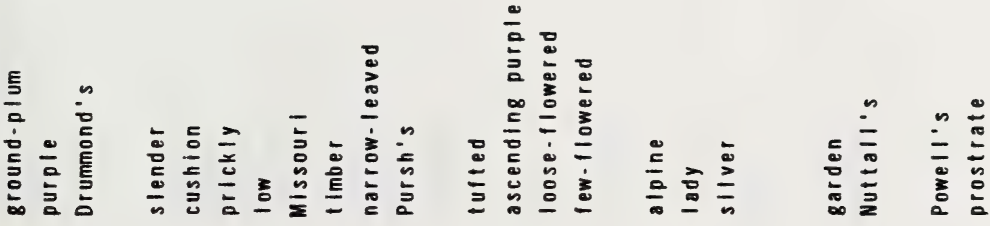

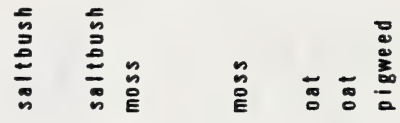

-
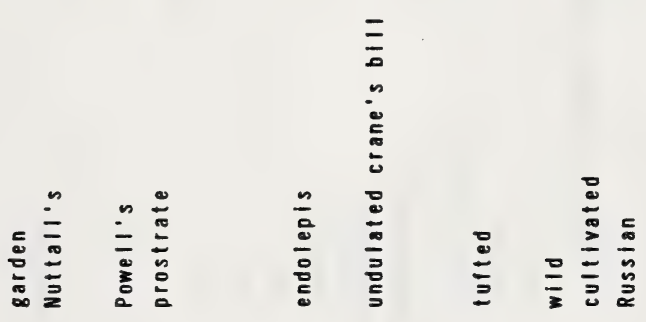

2

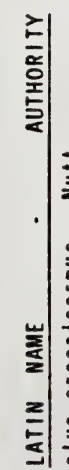

ำ

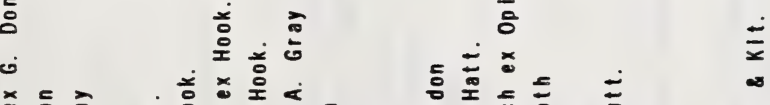

r.

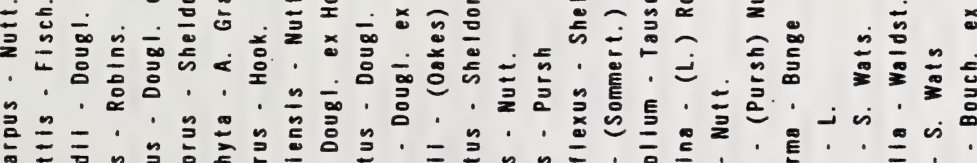

山ل几

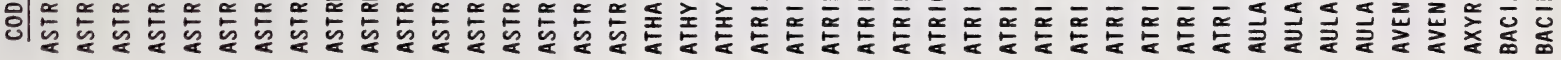




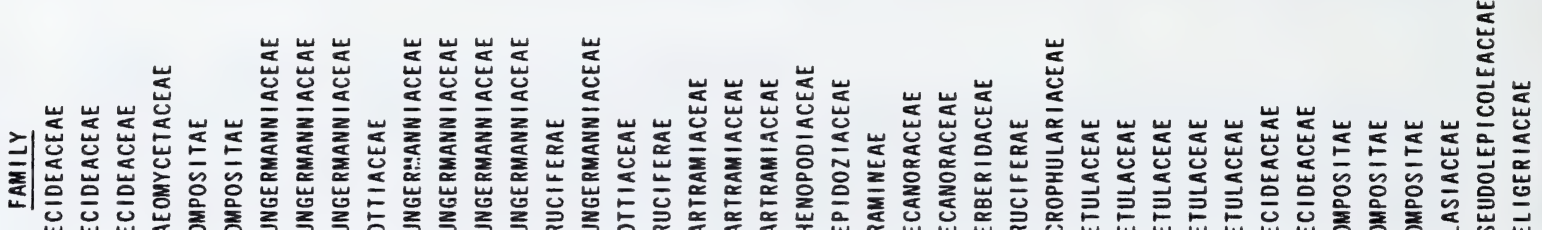

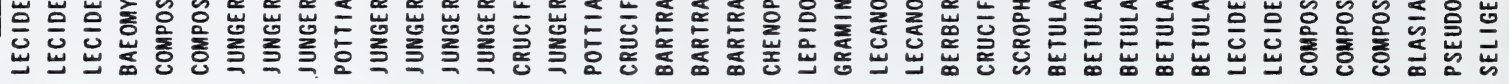

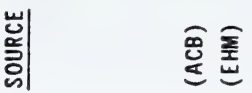
要
要金

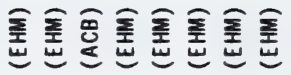
重寒雪
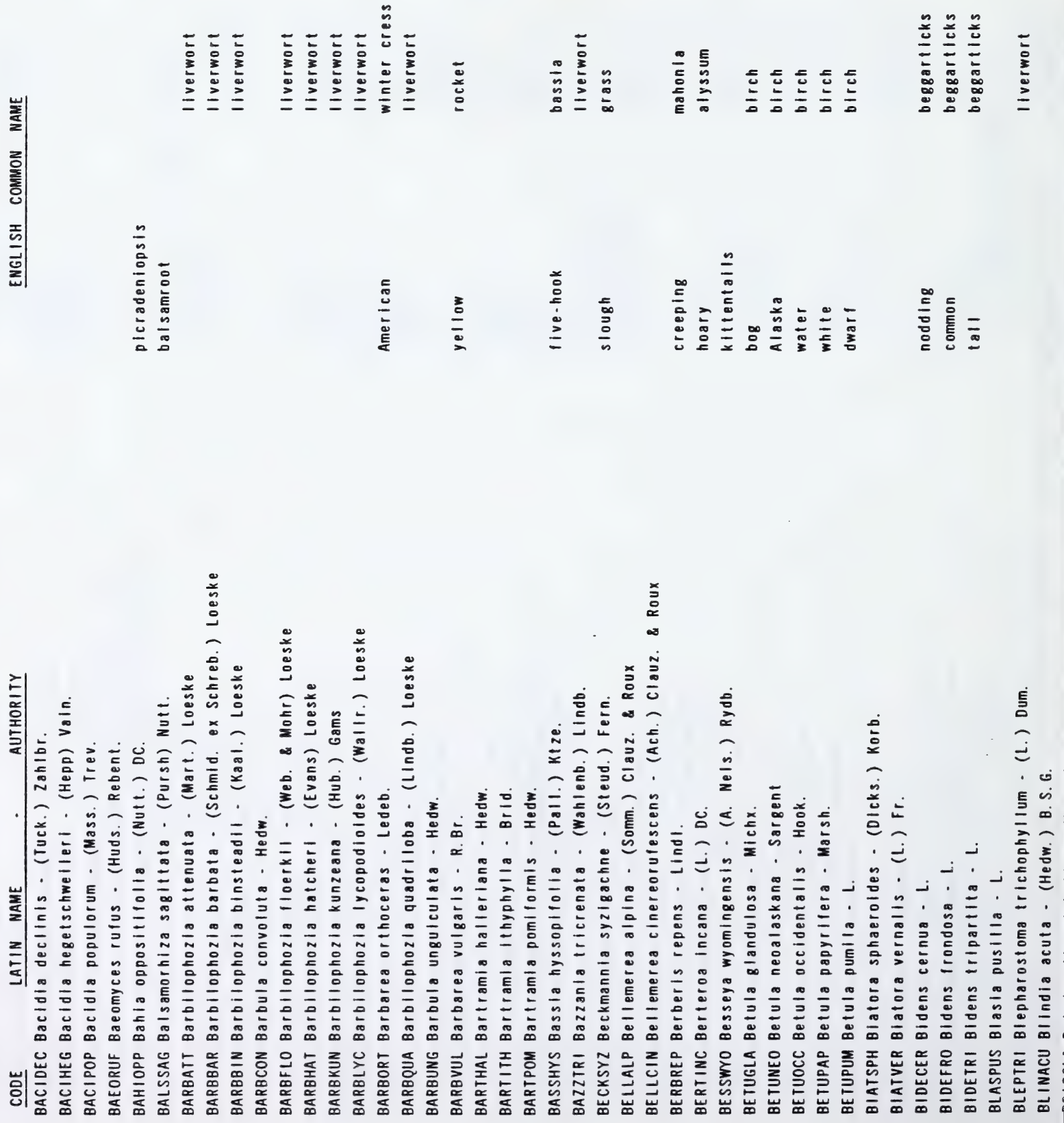


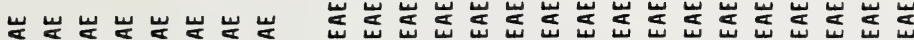

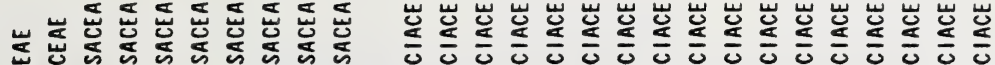

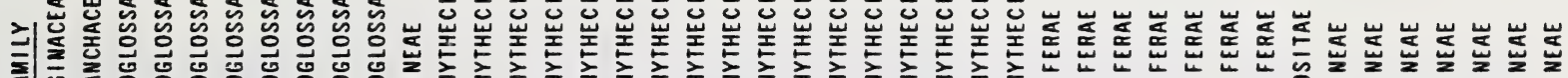

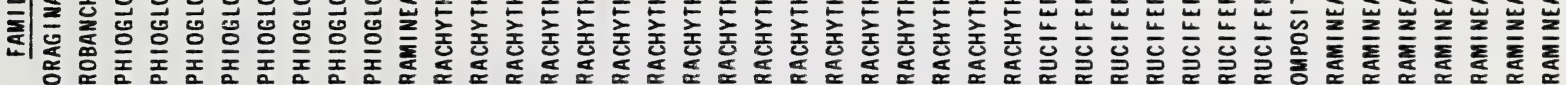

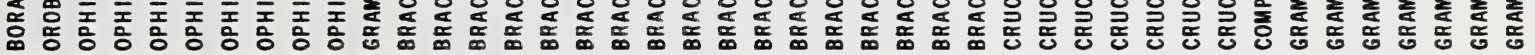

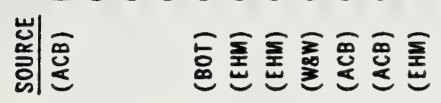

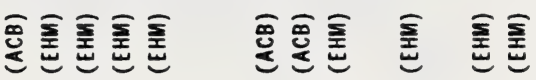

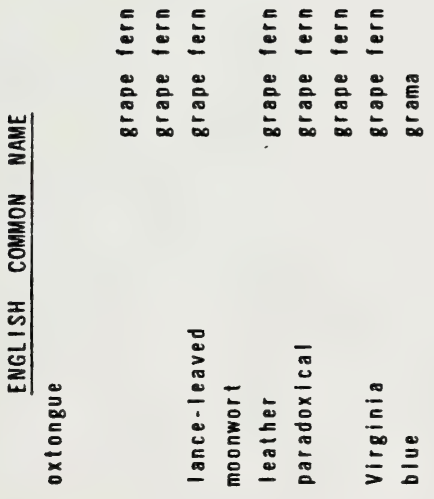

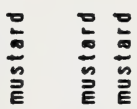

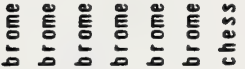<smiles>C#CC#CC</smiles>

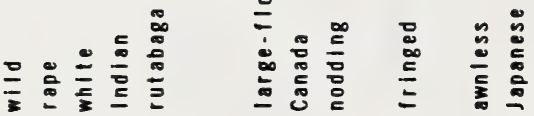

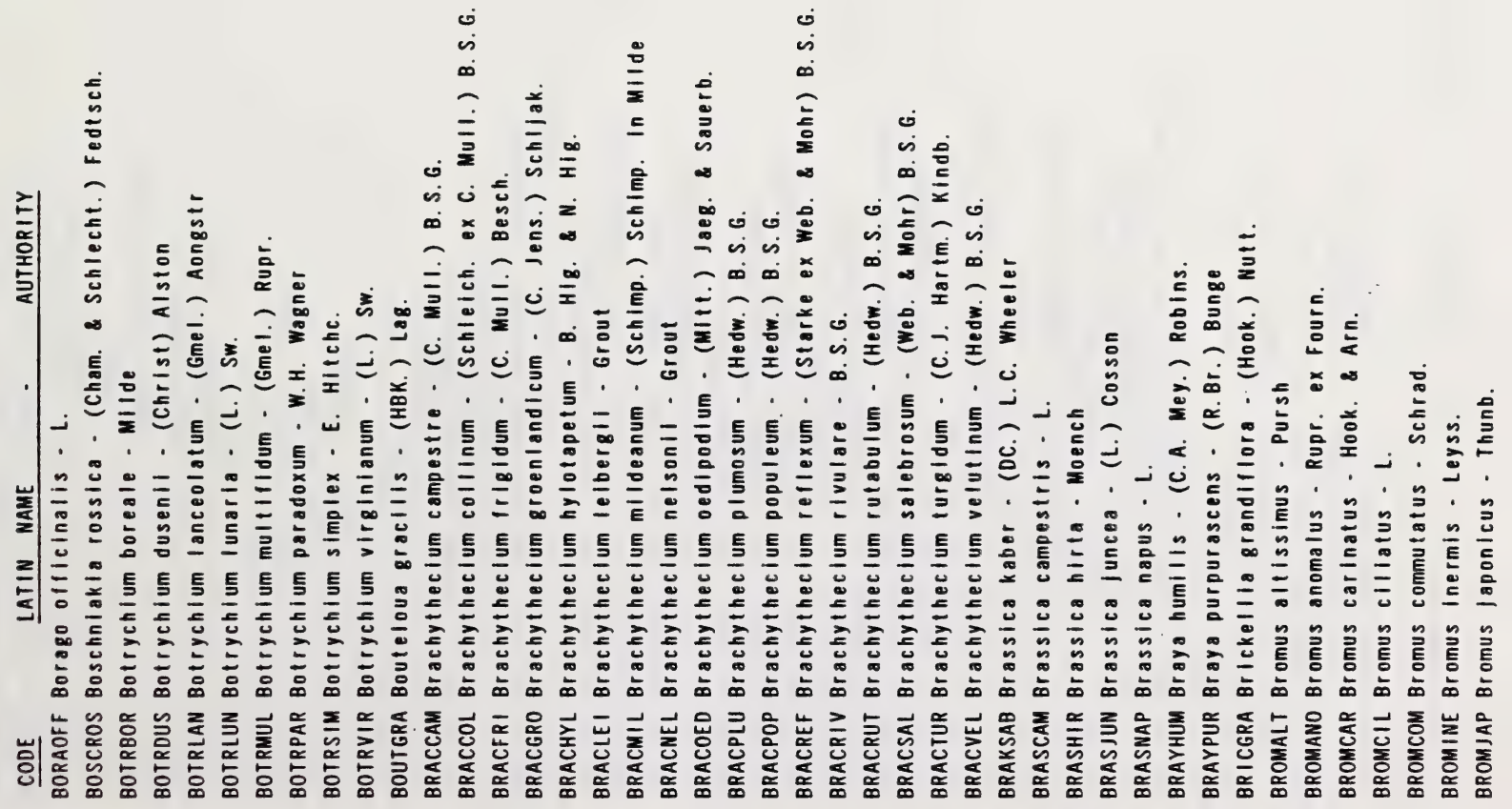


خ|

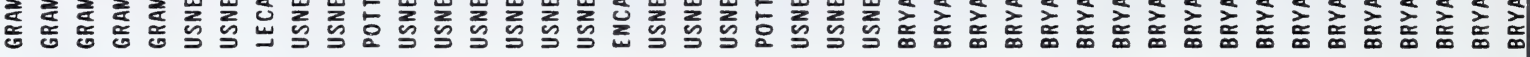

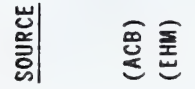

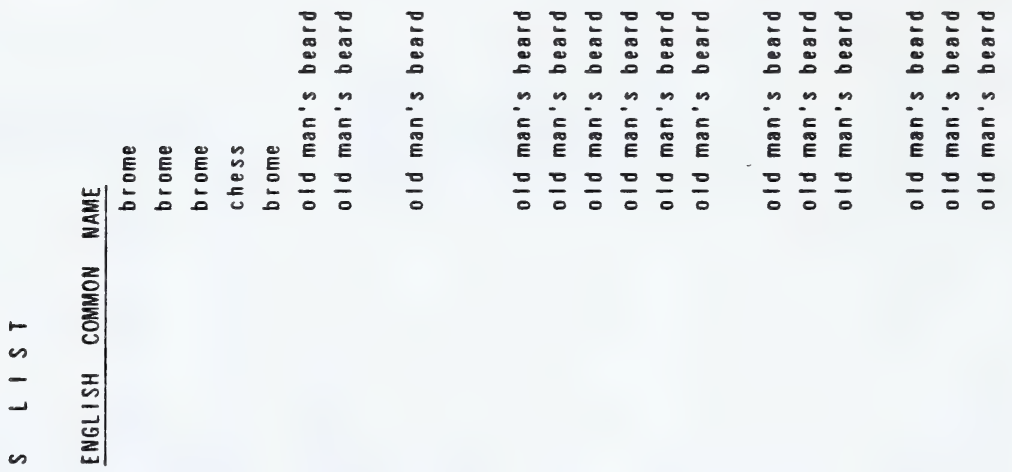

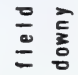

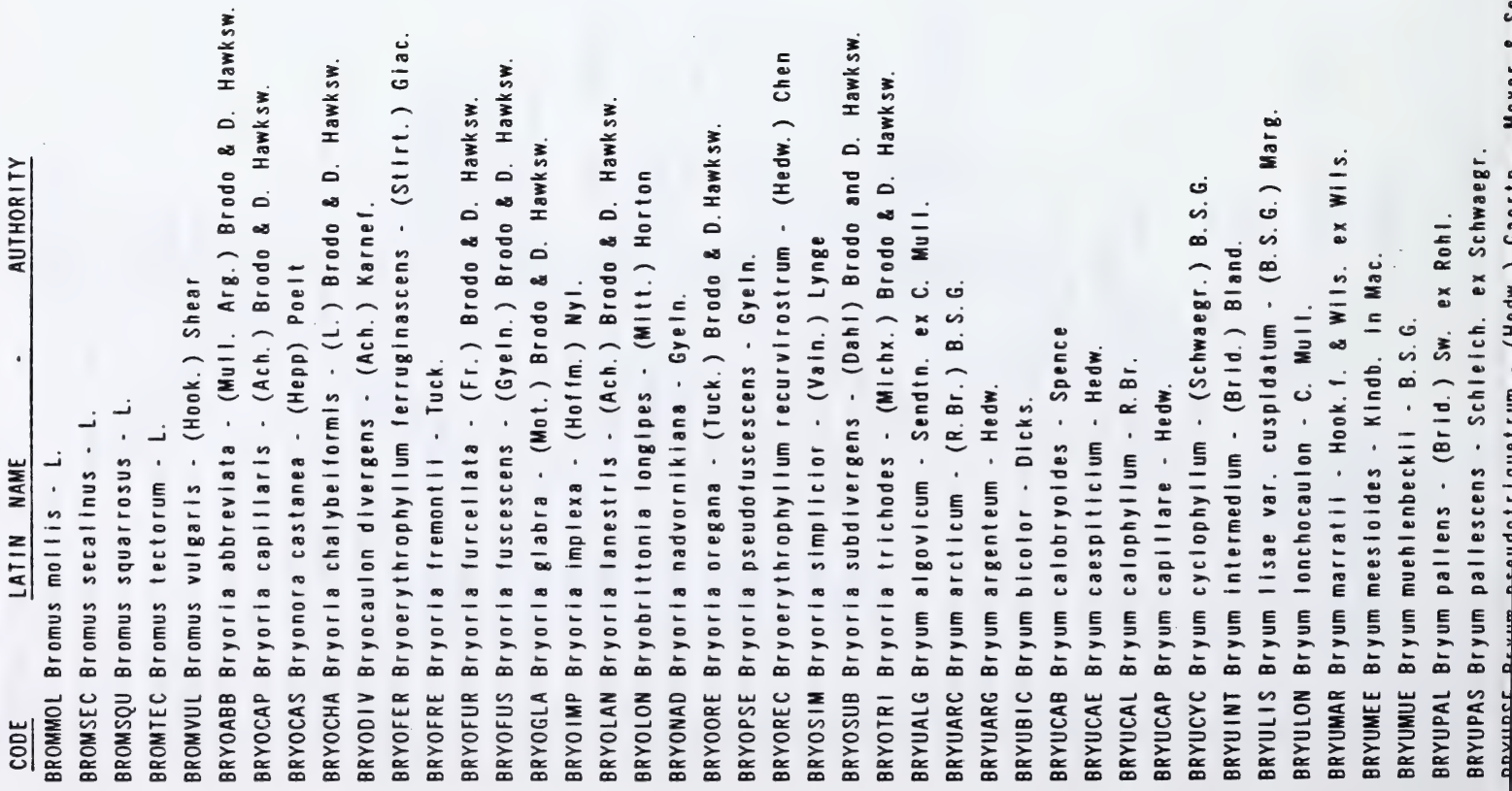


خإ

崖崖崖崖岕岕岕岕崖

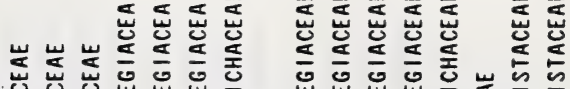

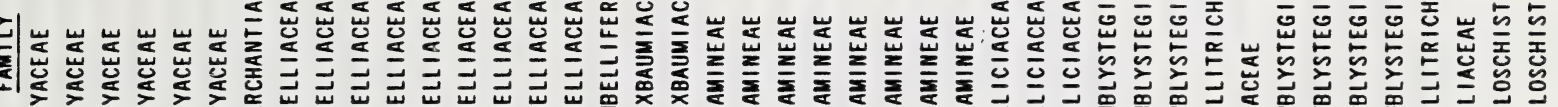
䓂|

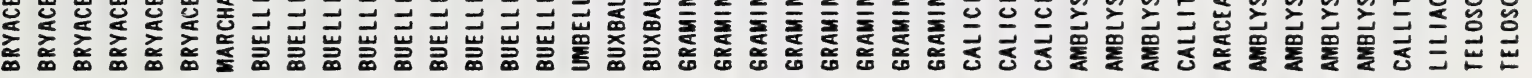

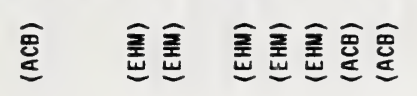

要要

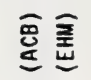

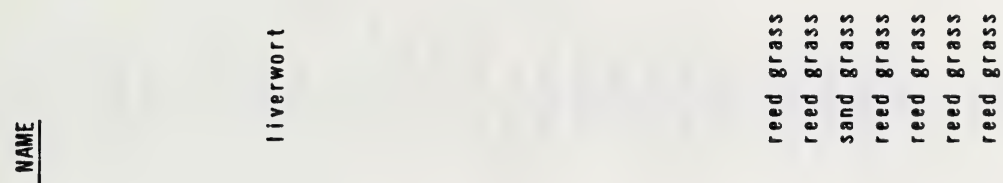

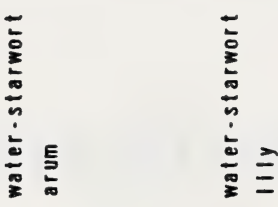

$\frac{2}{\frac{2}{2}}$

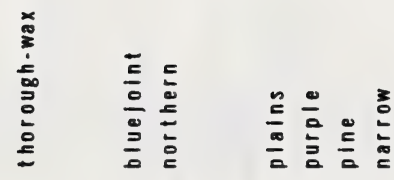

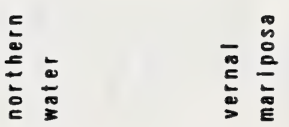

(n)

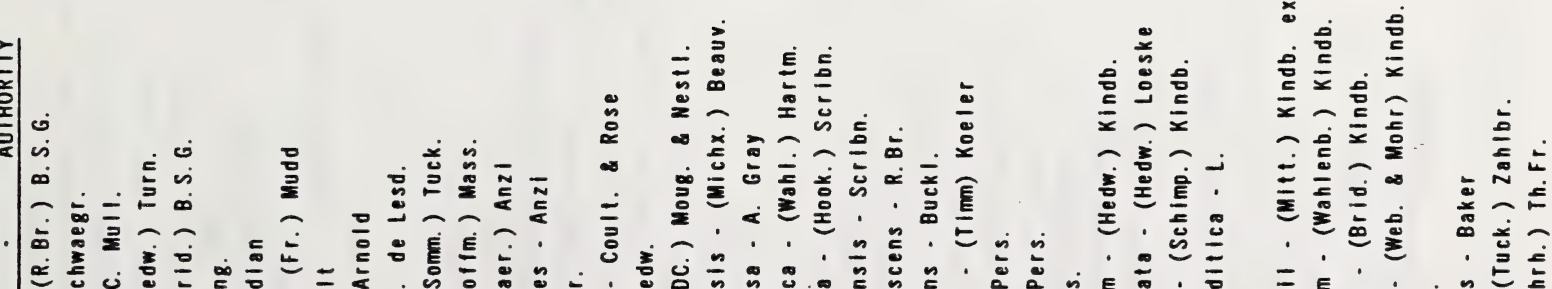

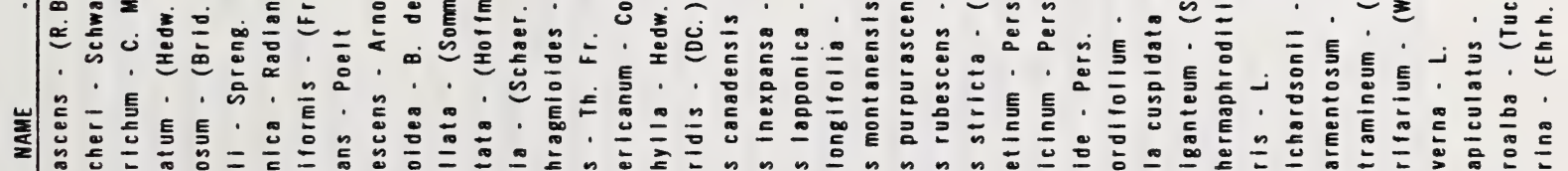

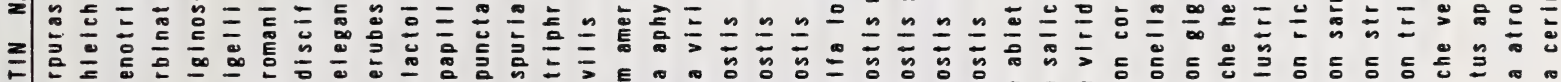

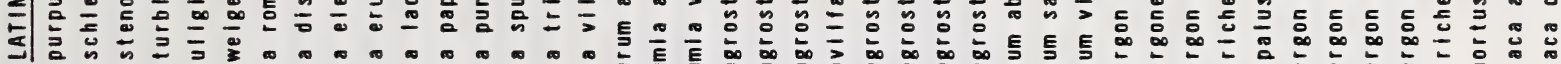

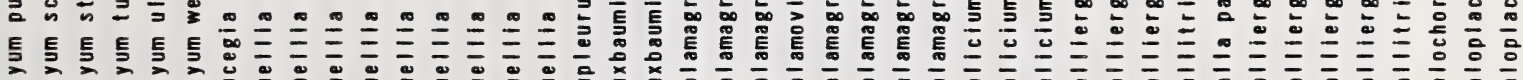

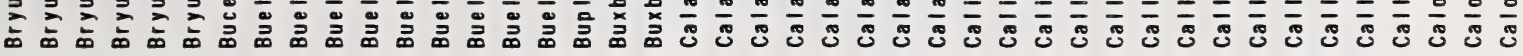

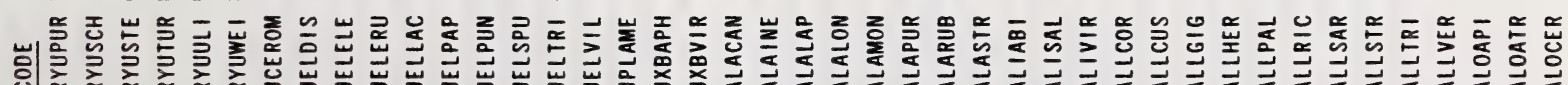

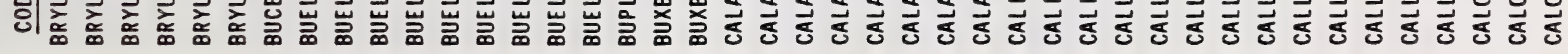




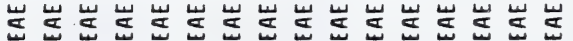

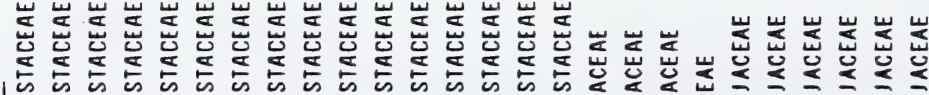

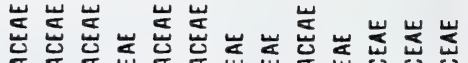

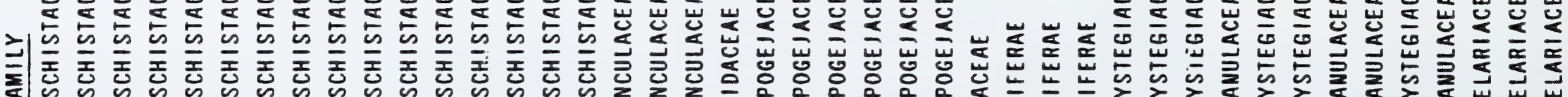

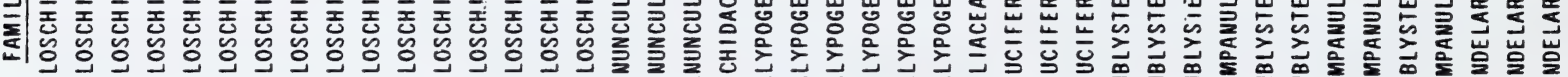

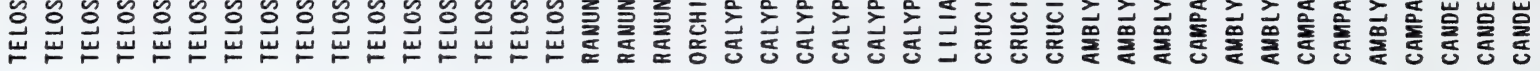
訔|

金要金要

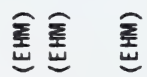

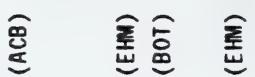

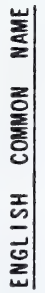

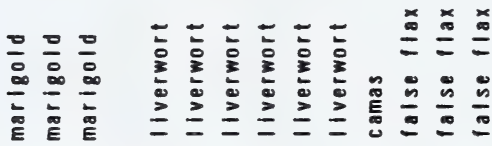

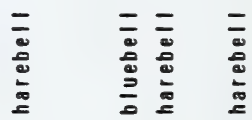

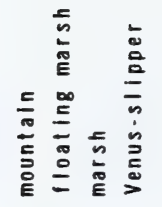

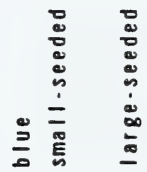

$\underset{\frac{\pi}{2}}{\frac{\pi}{\alpha}}$

$\underset{\substack{5 \\ 0}}{\substack{0 \\ \hdashline}}$

$\frac{\stackrel{a}{a}}{\frac{0}{0}}$

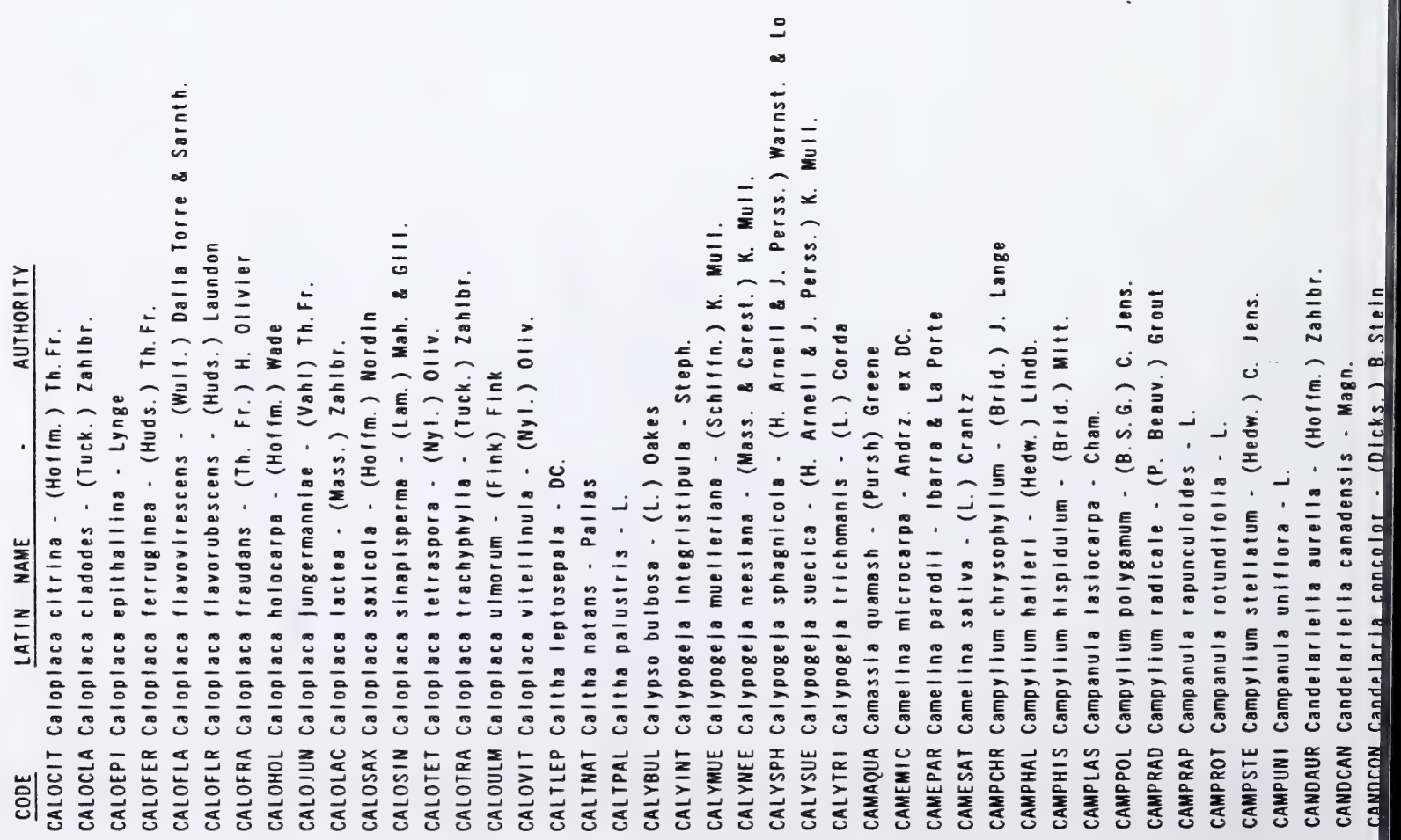


峞峞岩峞

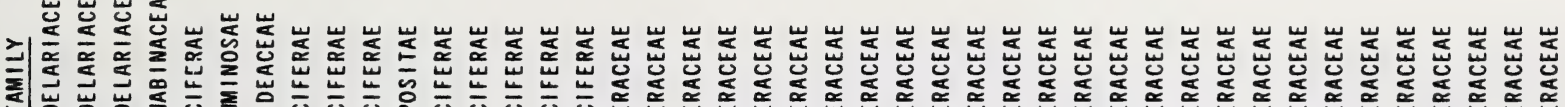

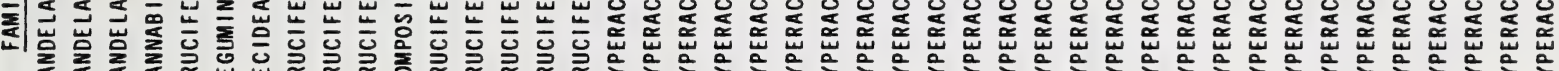

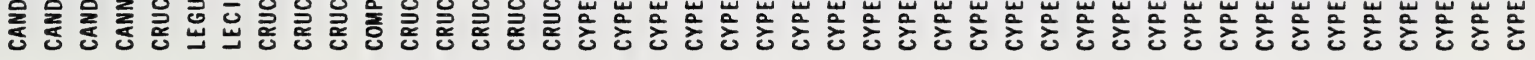

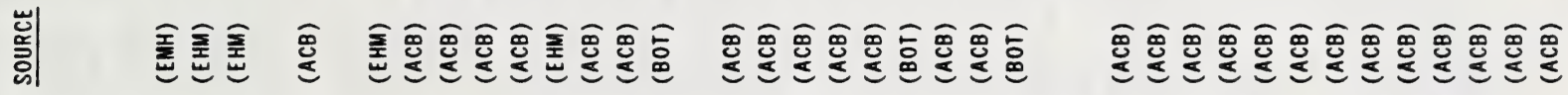

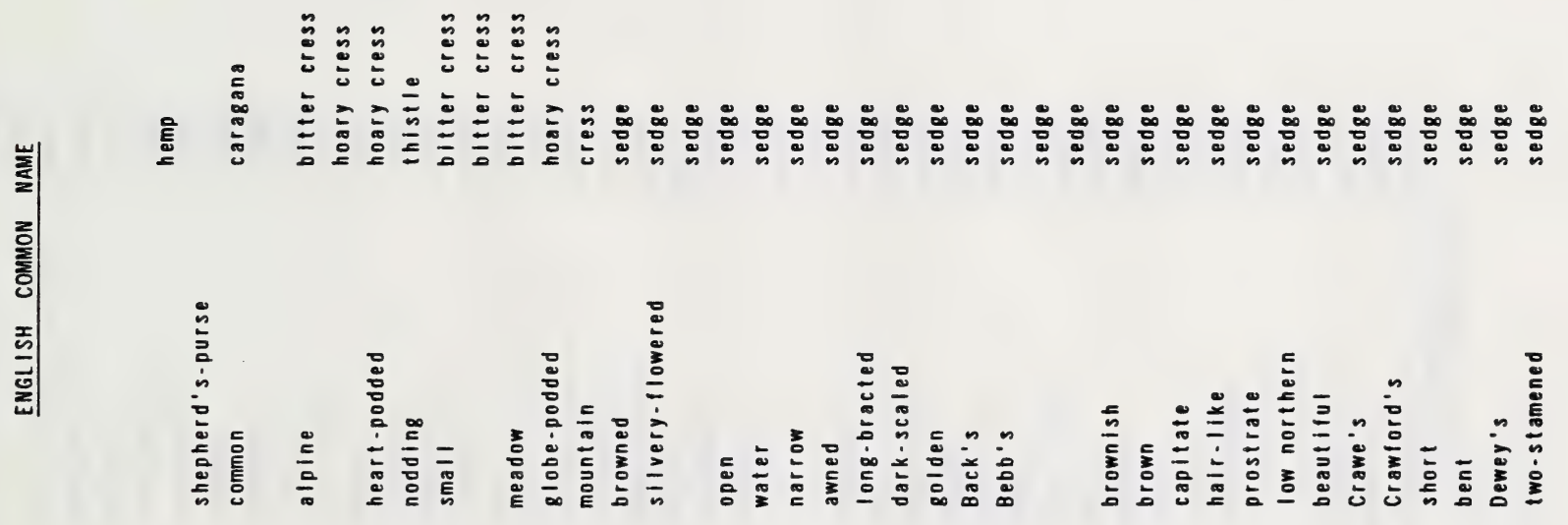

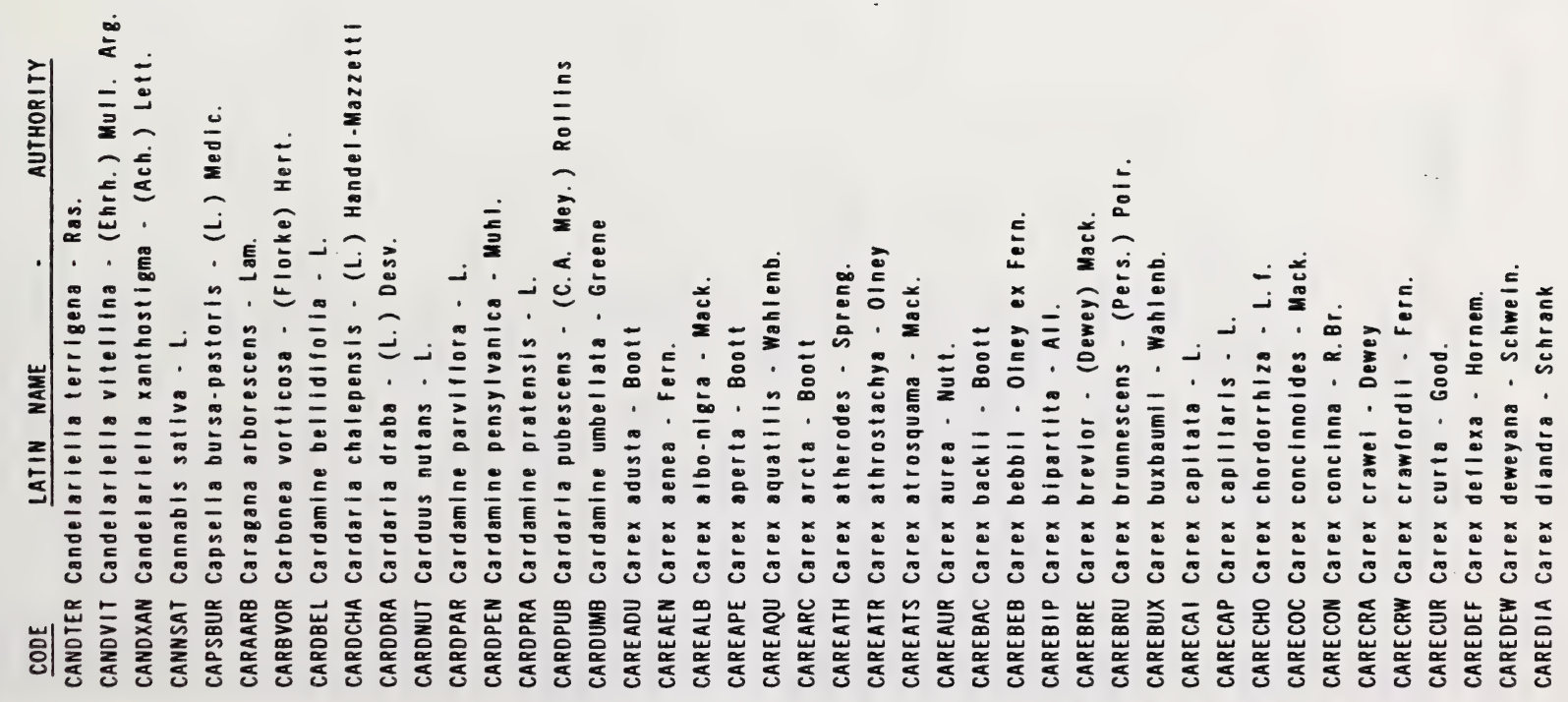




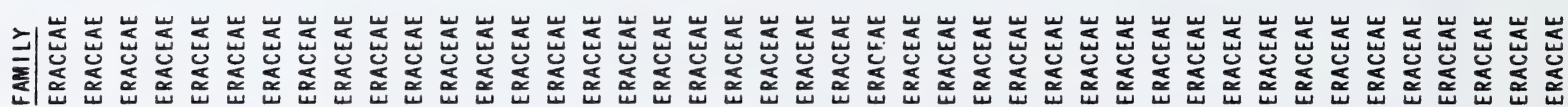

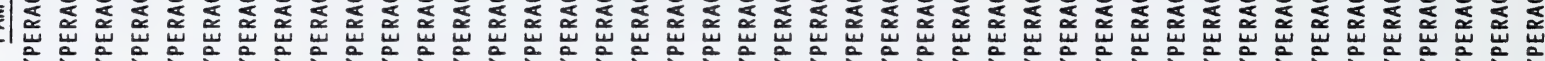

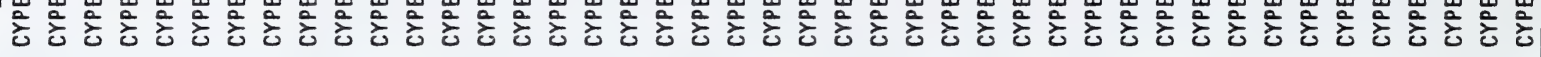

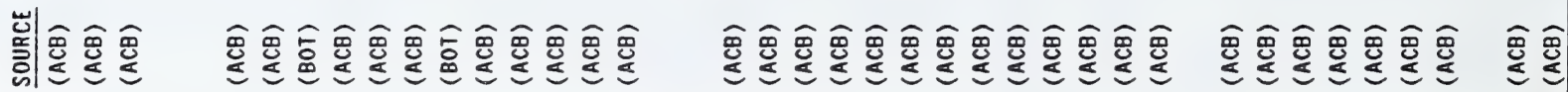

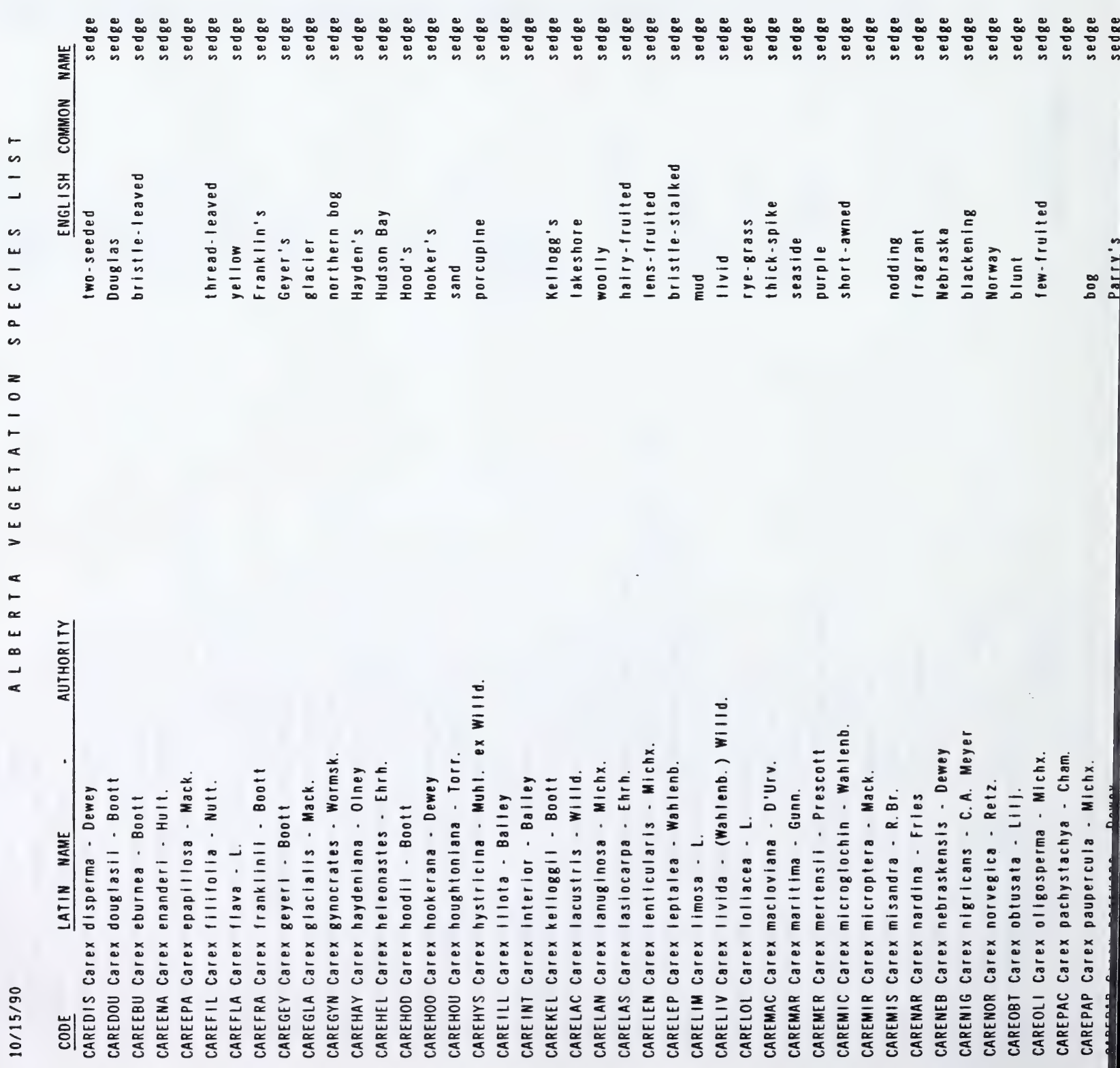




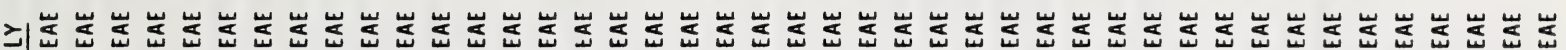
|⿹

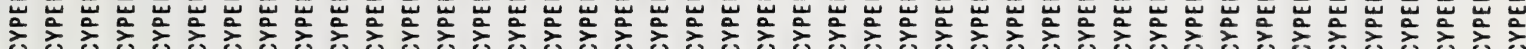

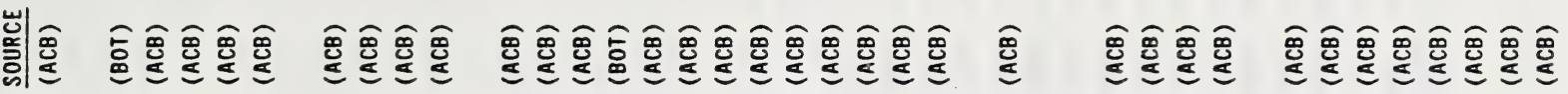

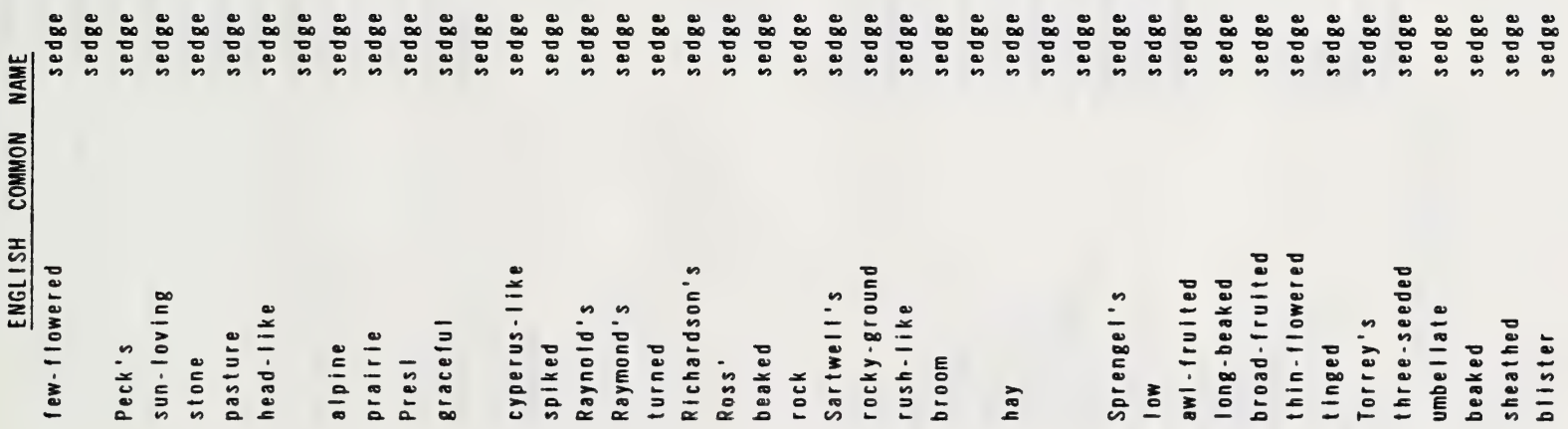

칳

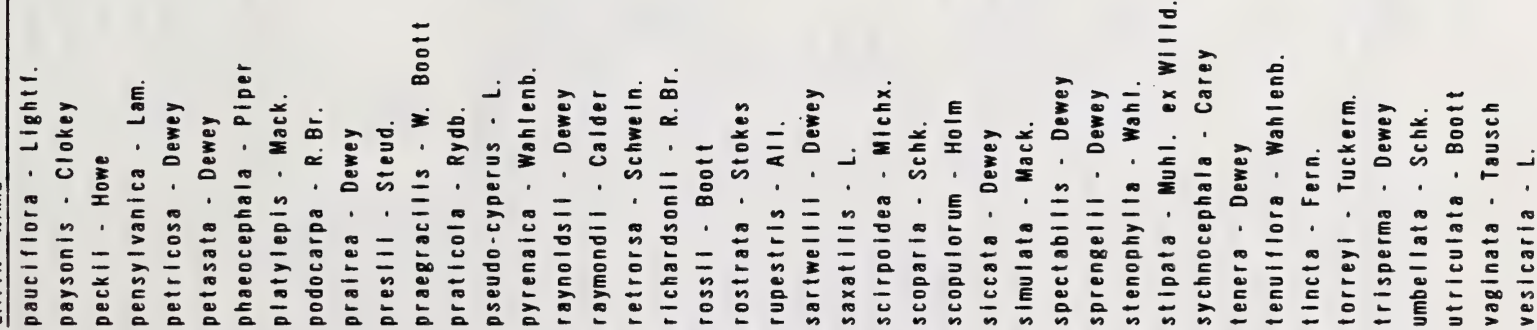

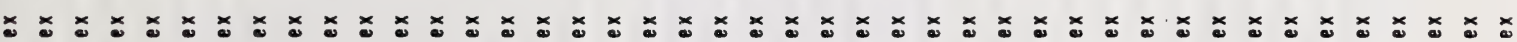

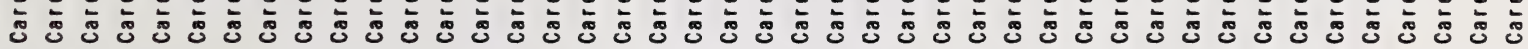

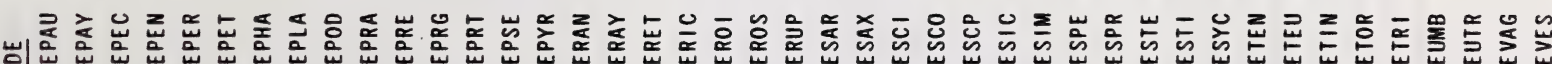

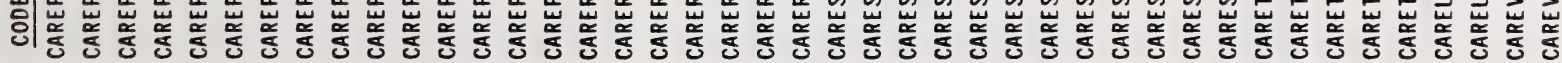




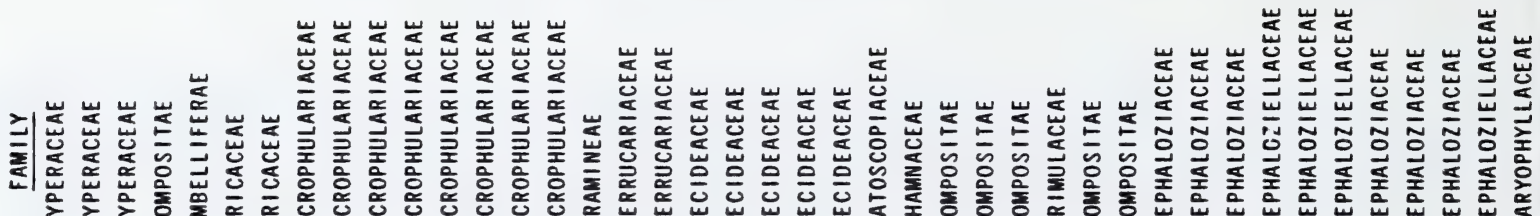

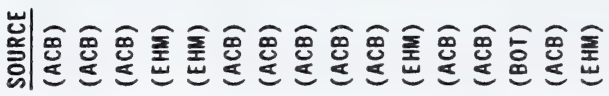

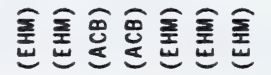

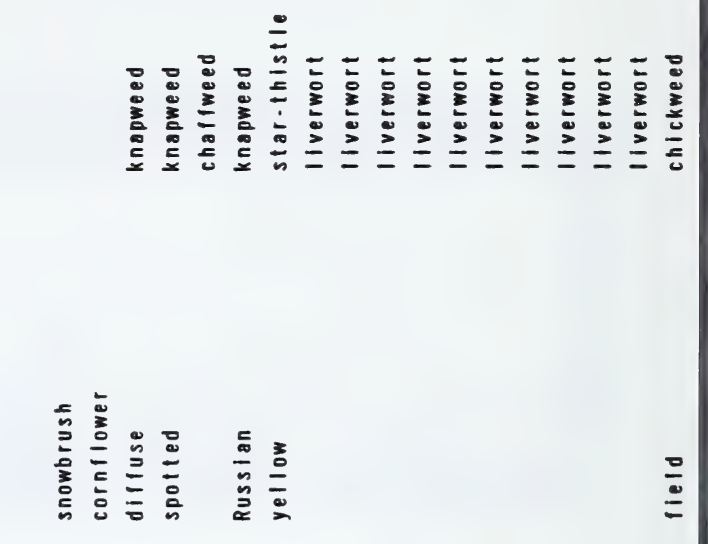

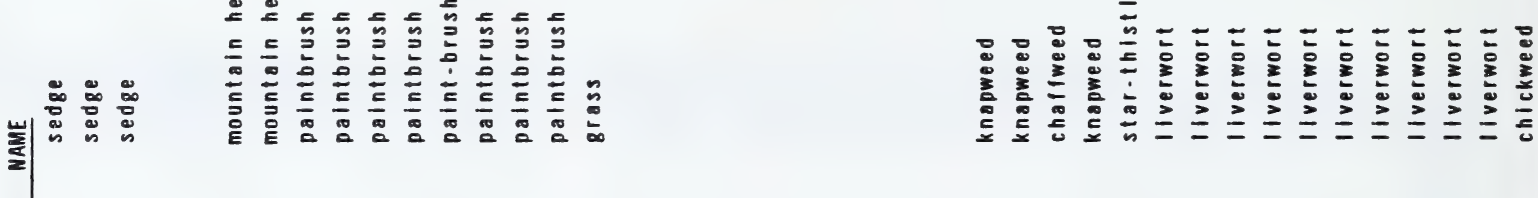

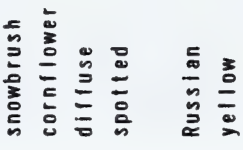
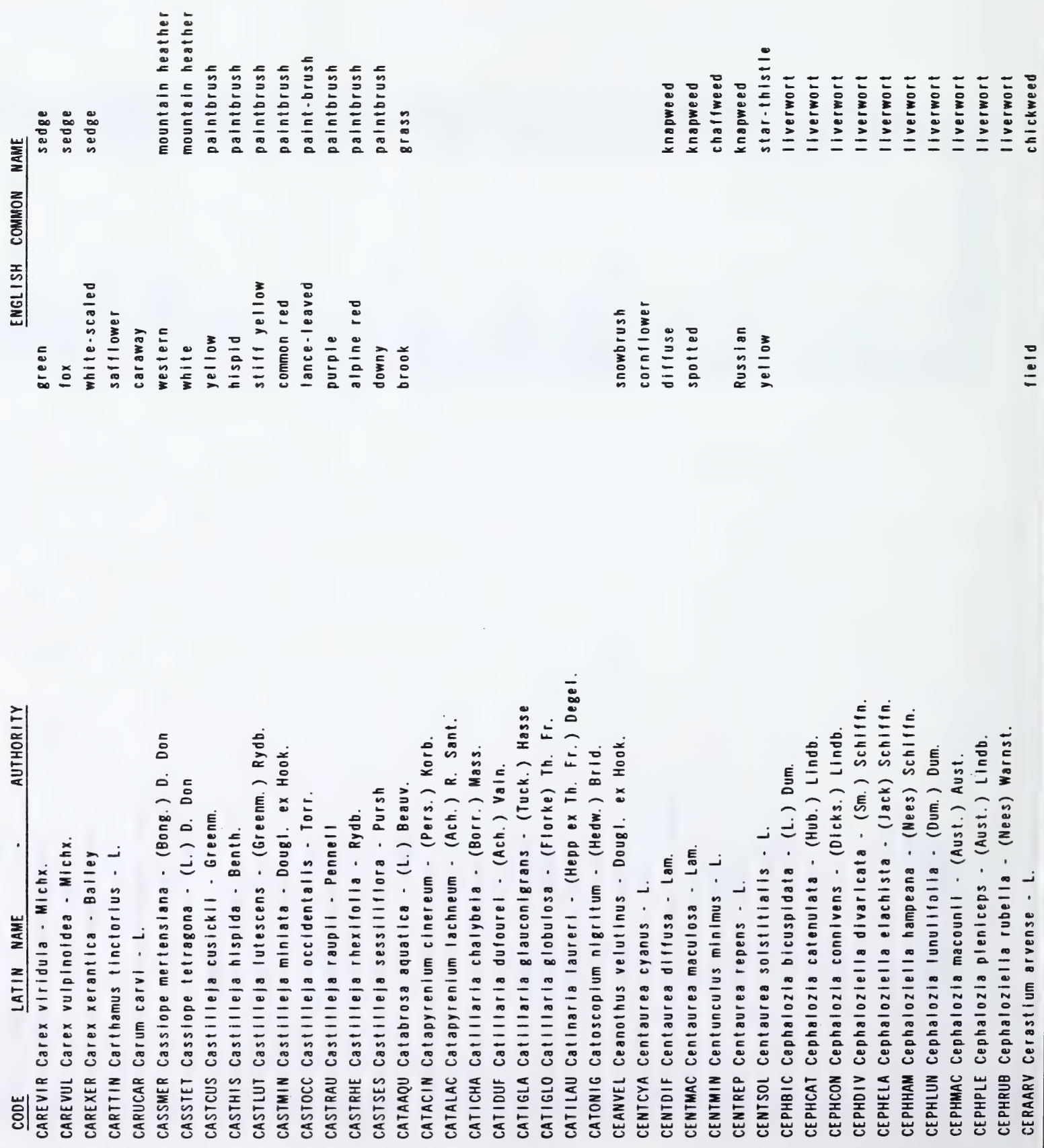


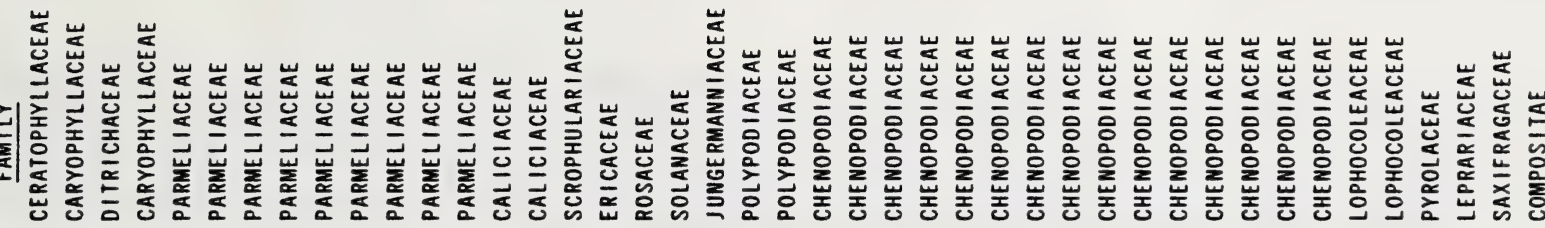

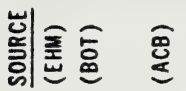

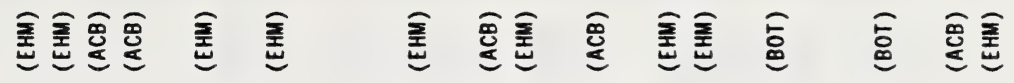
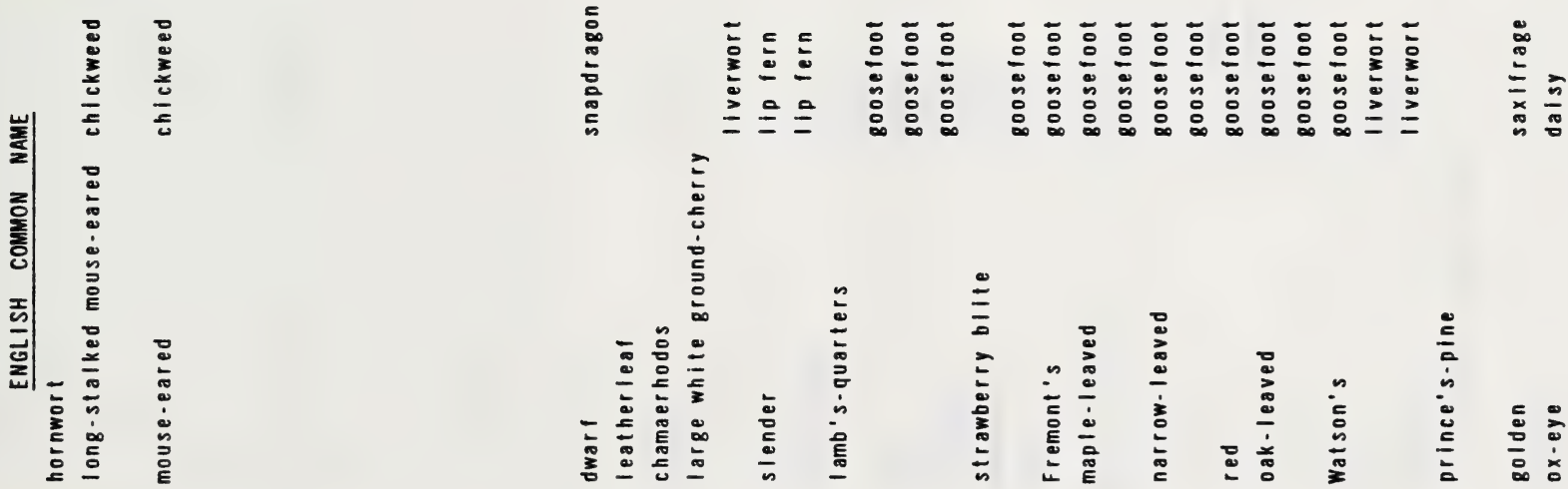

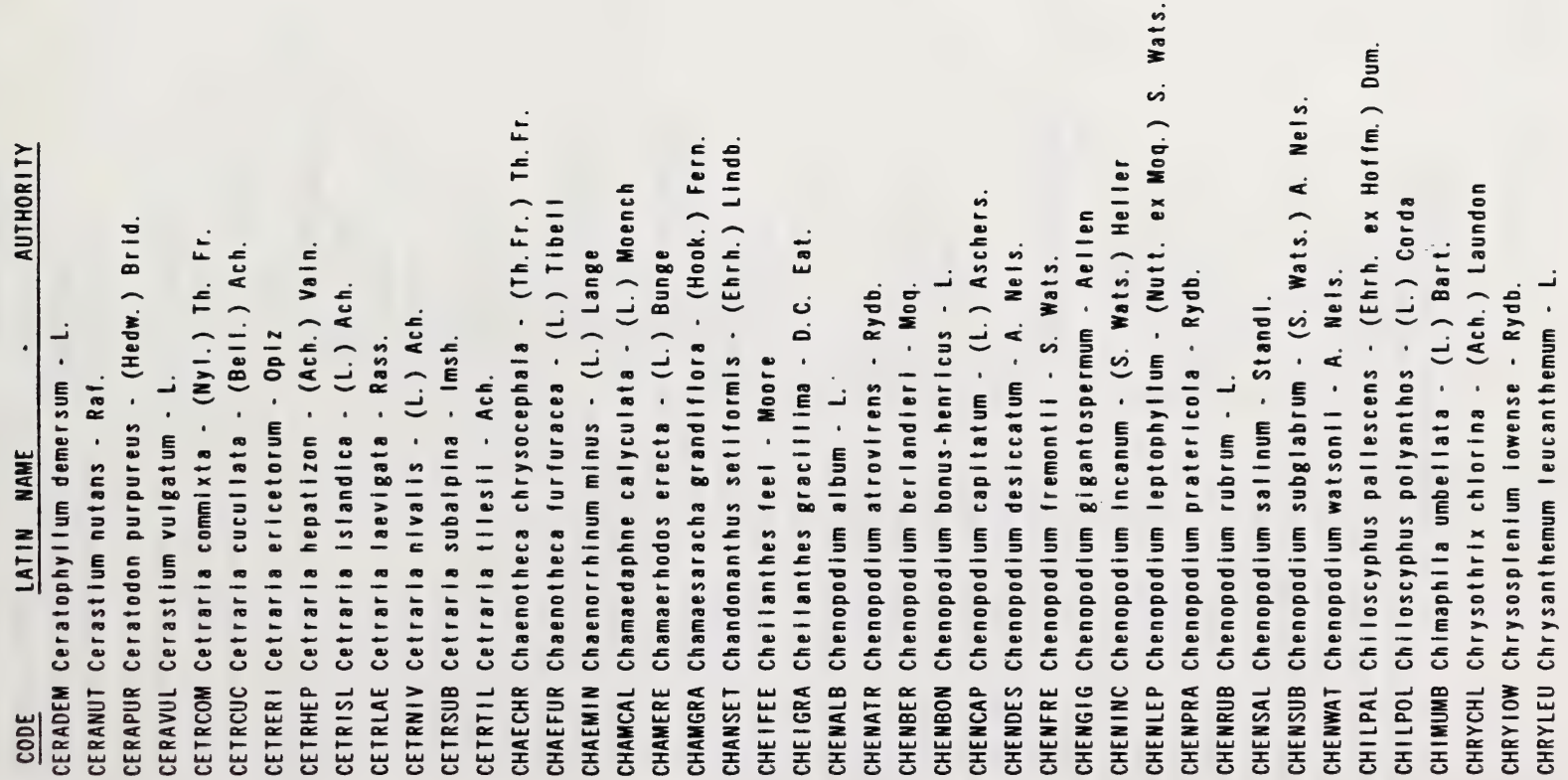




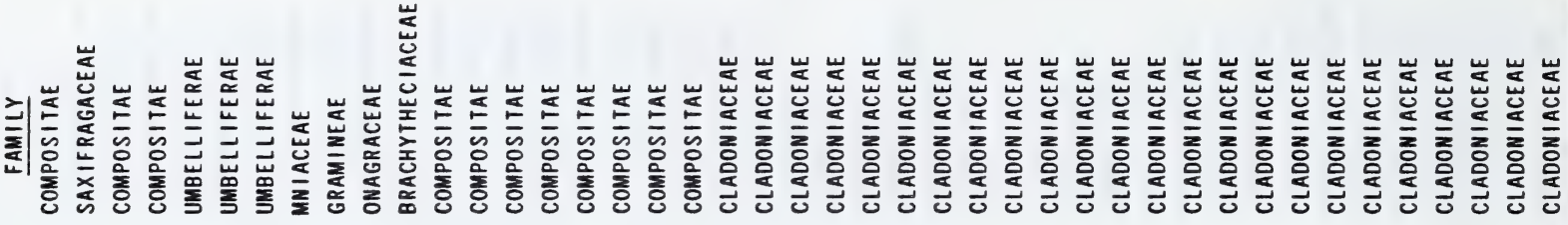

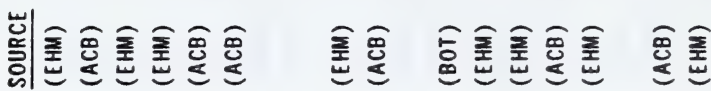

E
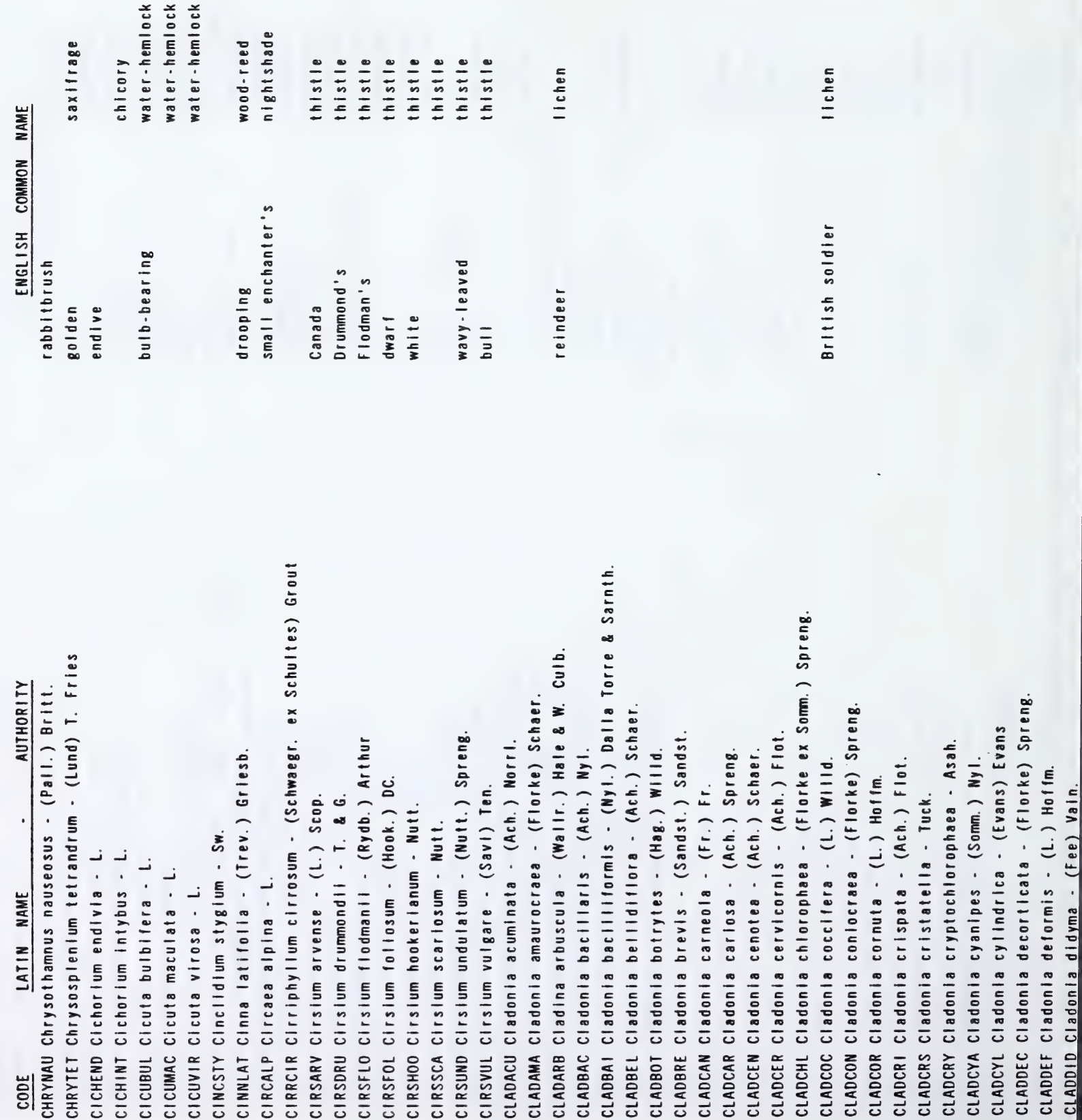


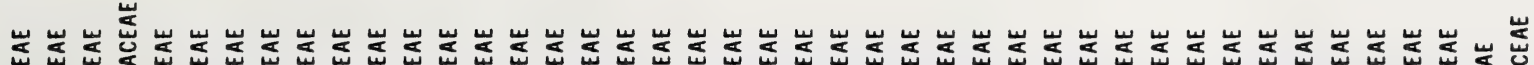

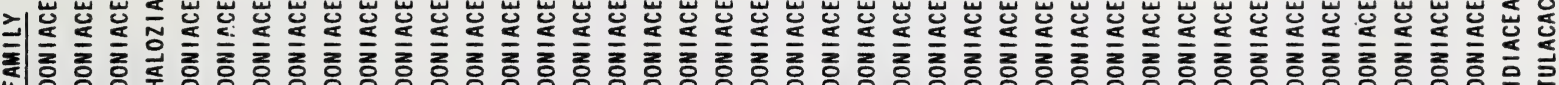

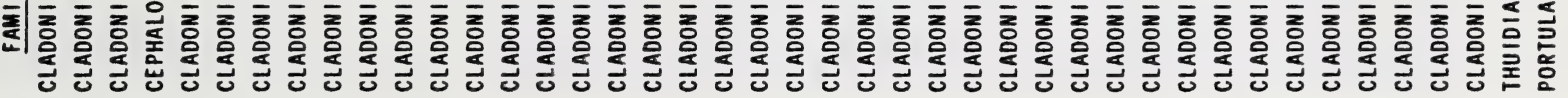

产|

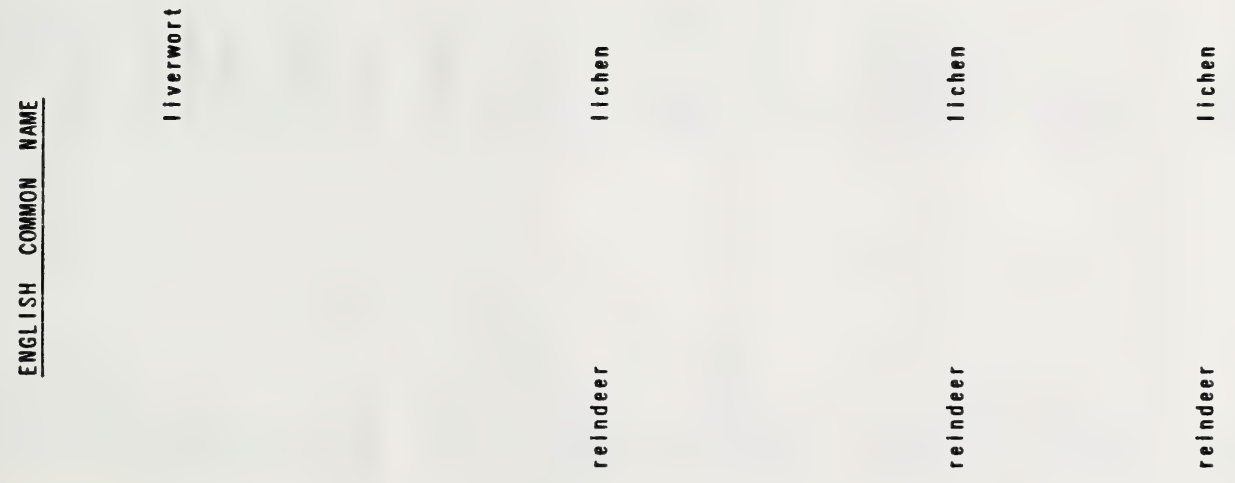




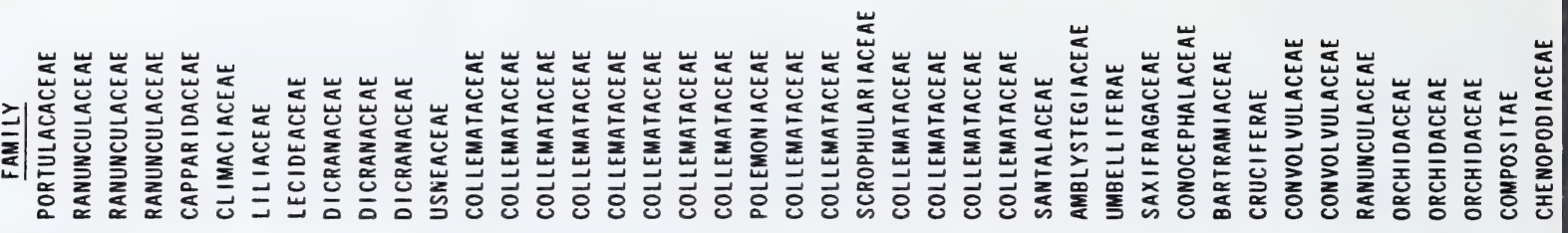

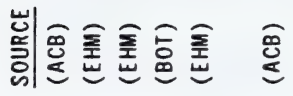

咅

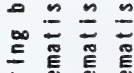

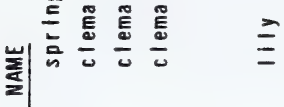

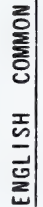

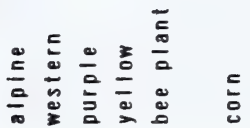

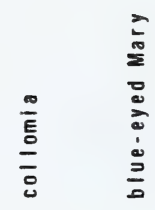

$\overbrace{\substack{0 \\ 0}}^{\frac{5}{0}} \frac{5}{0}$

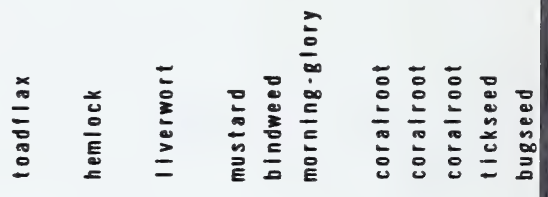

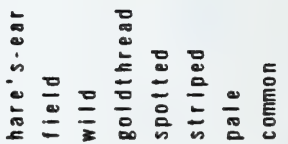

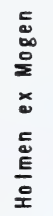

引)

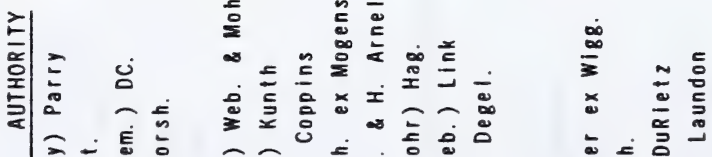

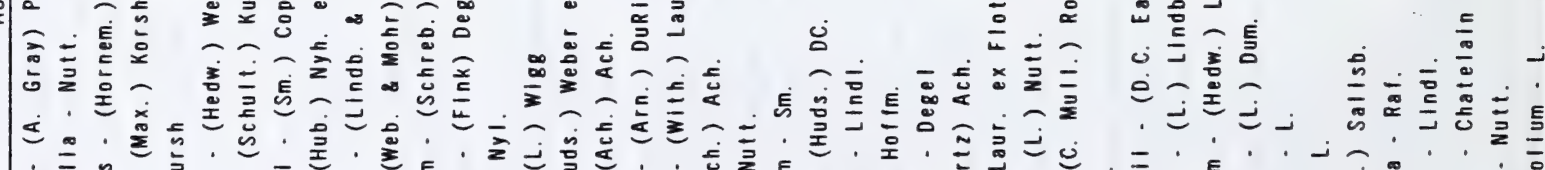

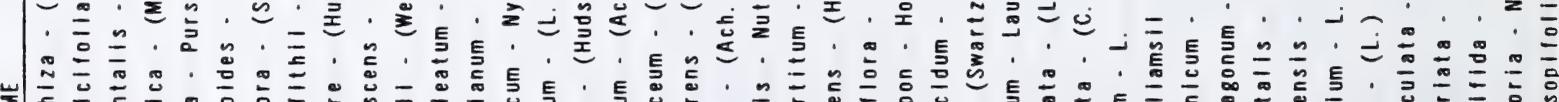

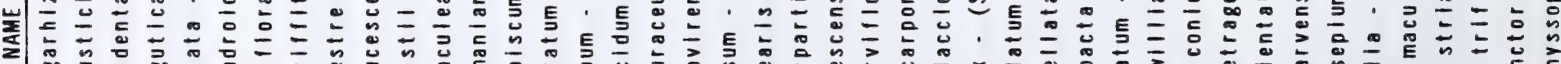

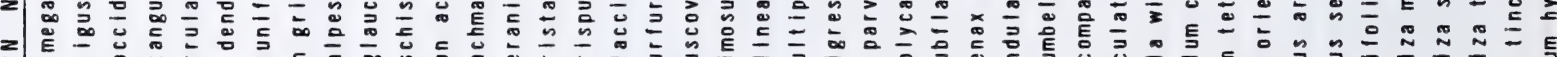

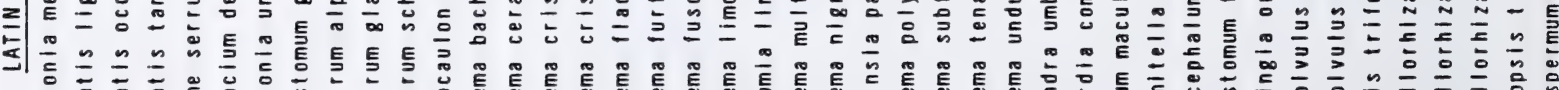

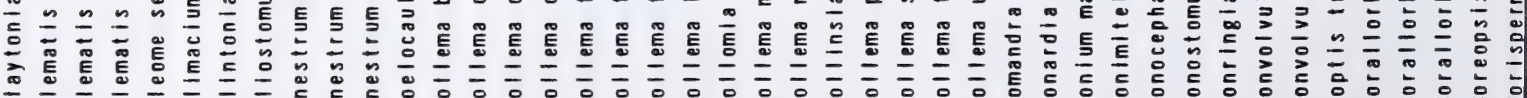

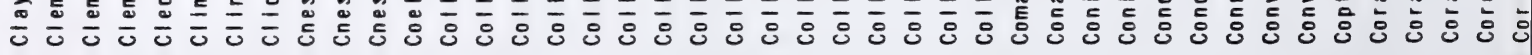

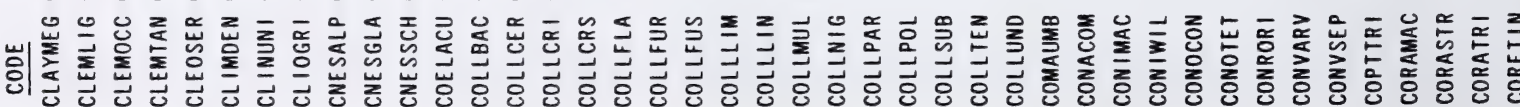




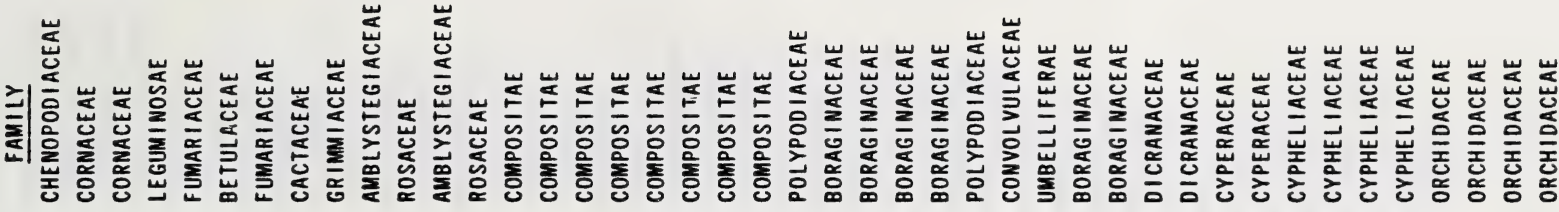

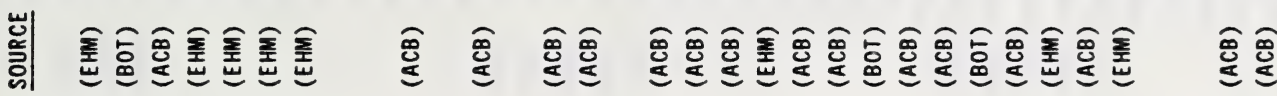

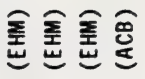

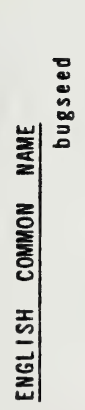

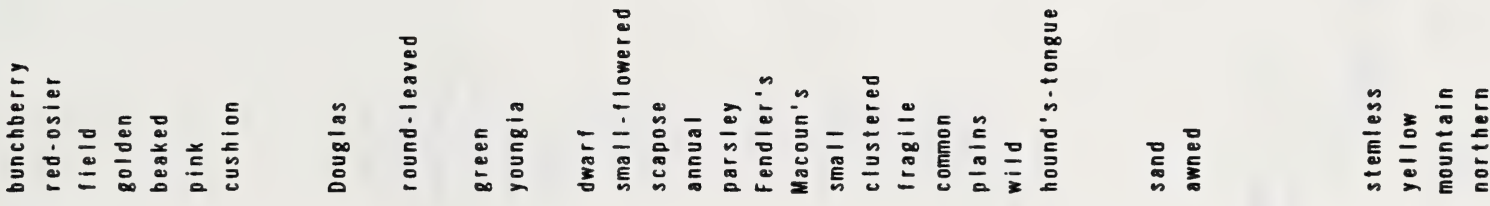

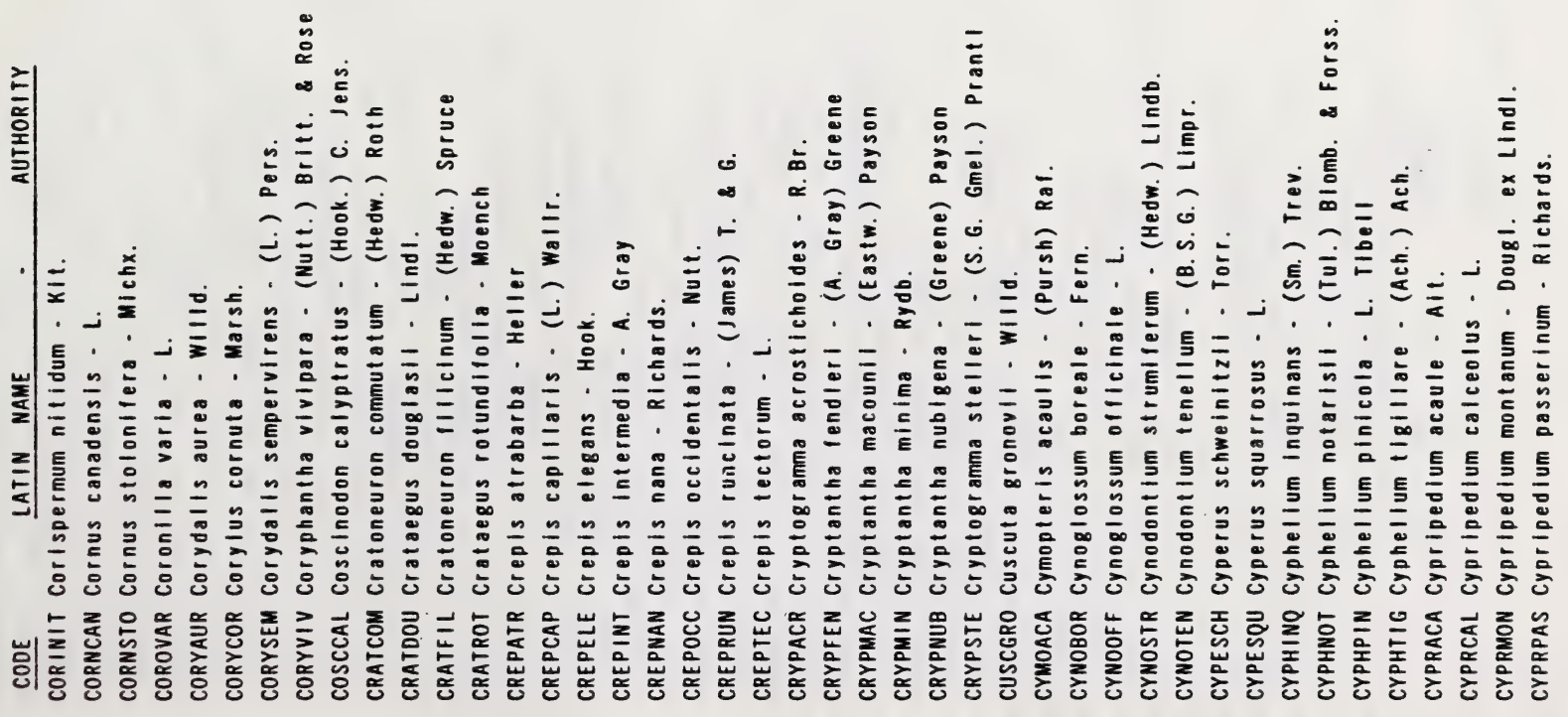




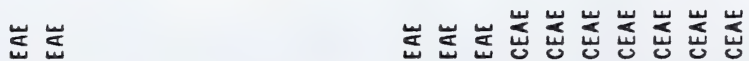
引|山辶大

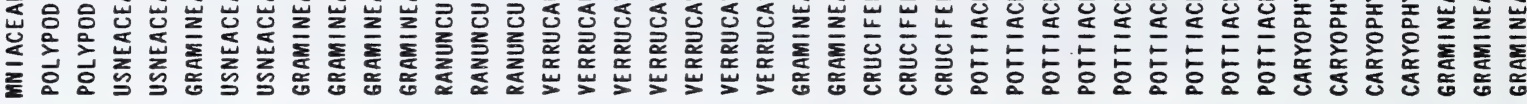

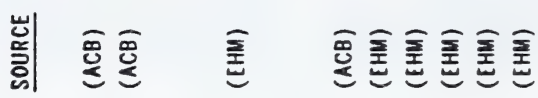

金金尊金重

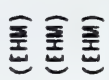

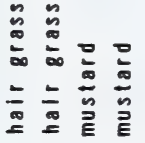

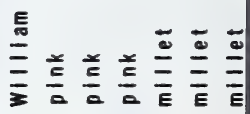

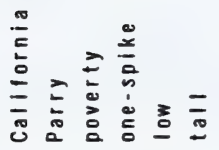

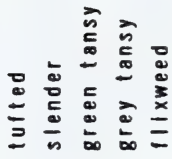

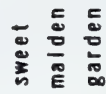

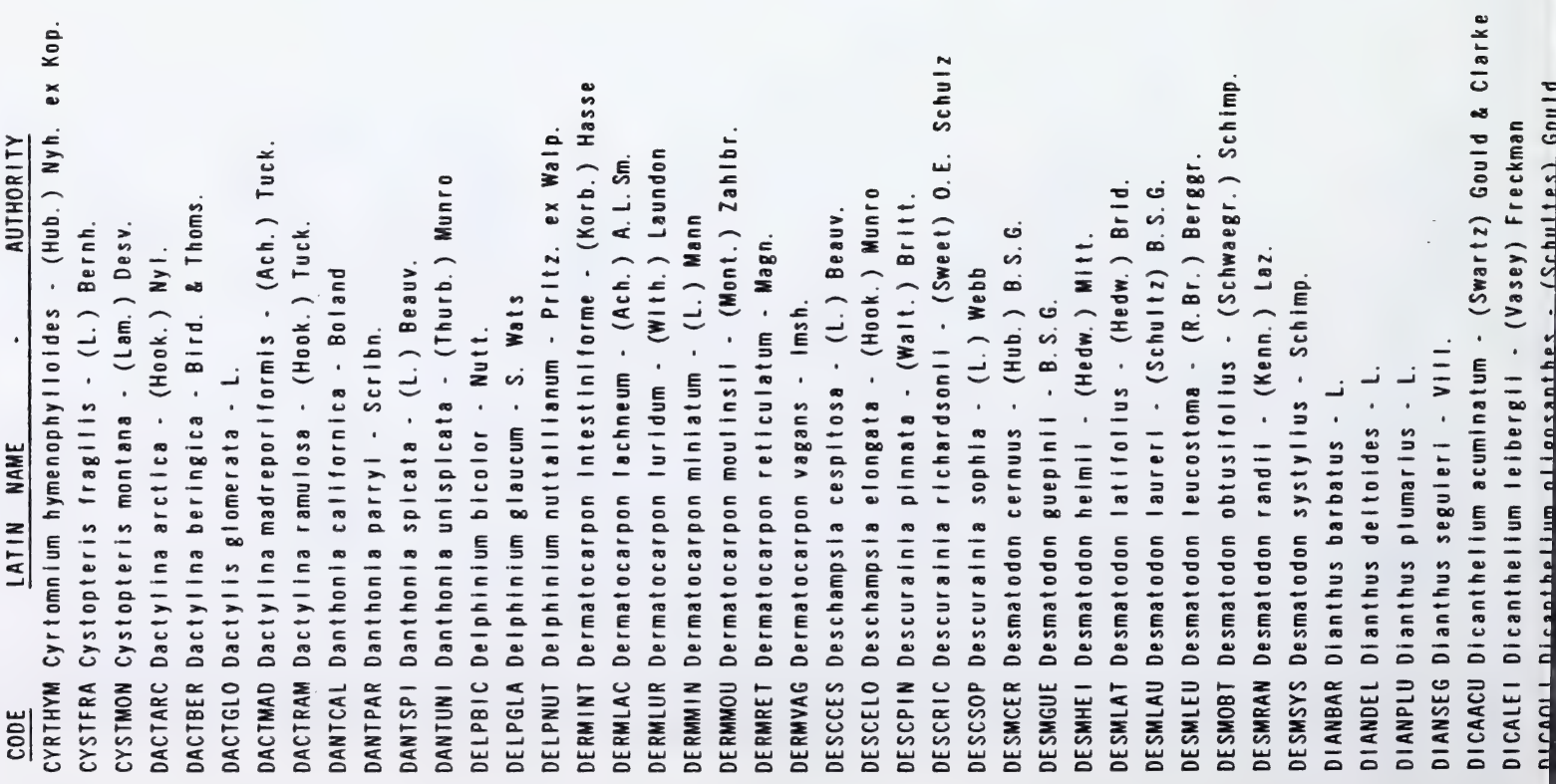




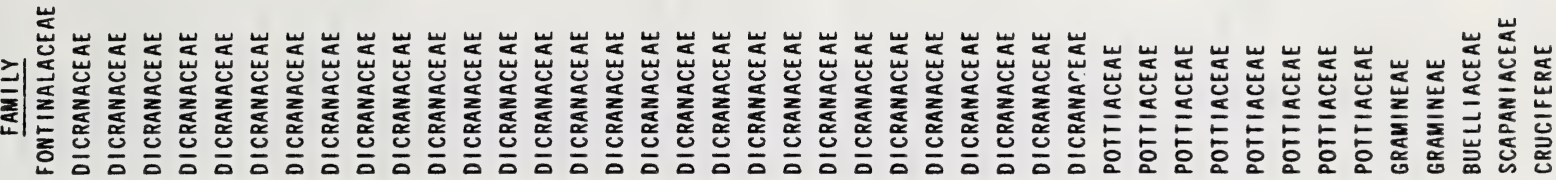
ㅎّㅎ|

总悆言

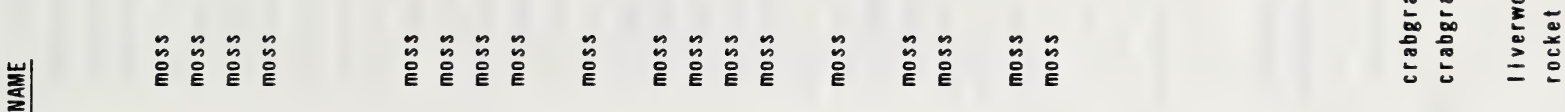

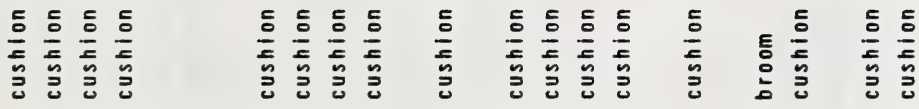

Е

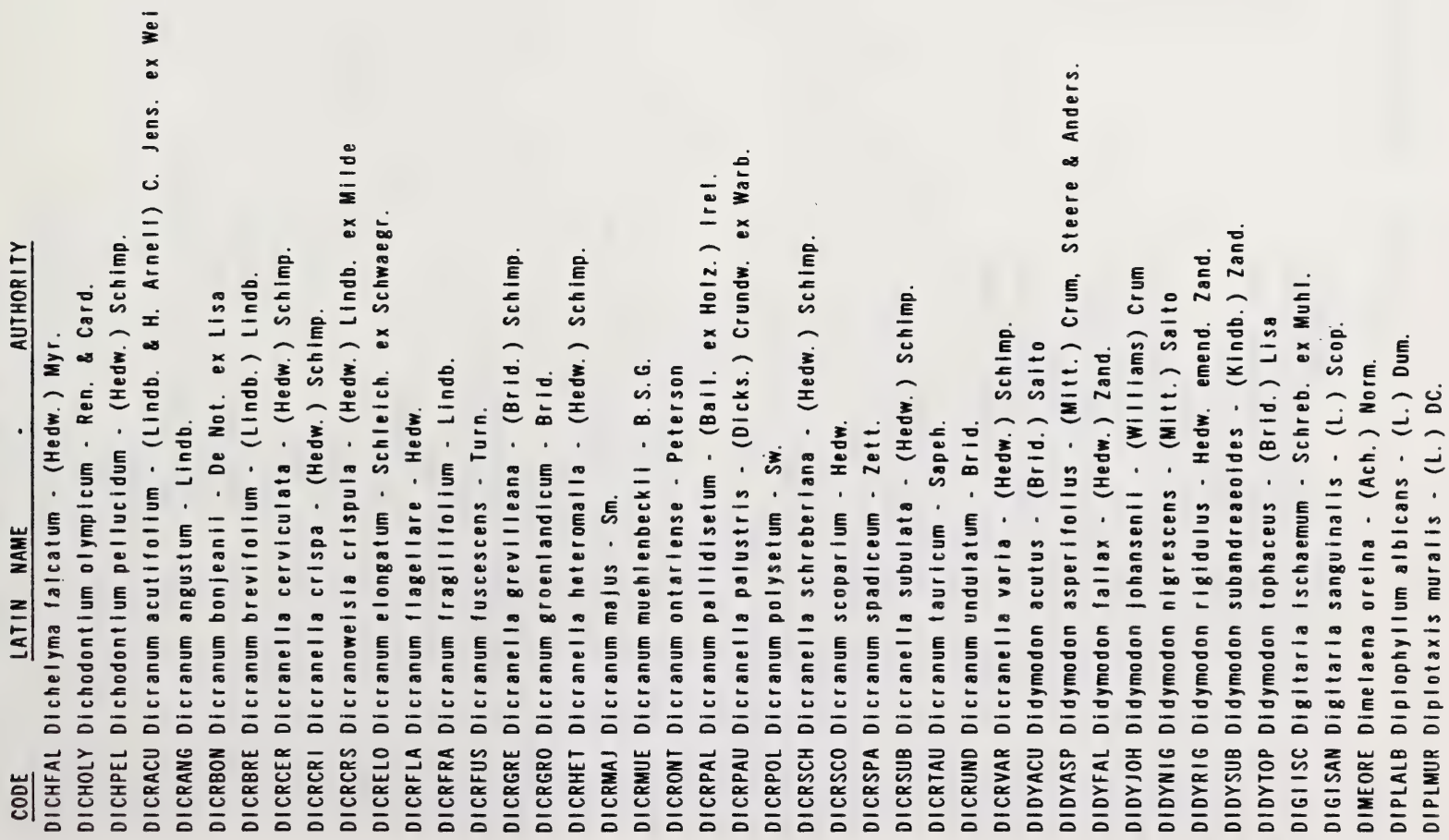




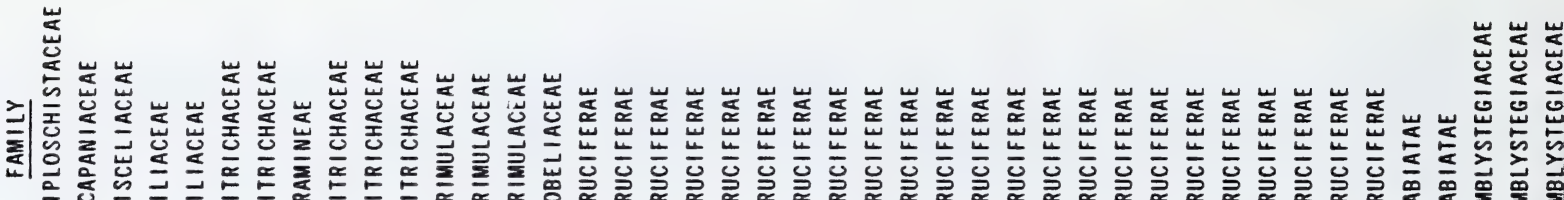
世

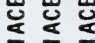
可 는

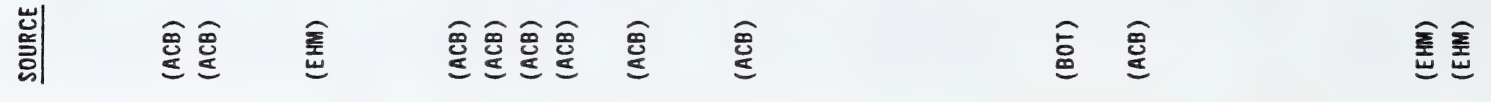

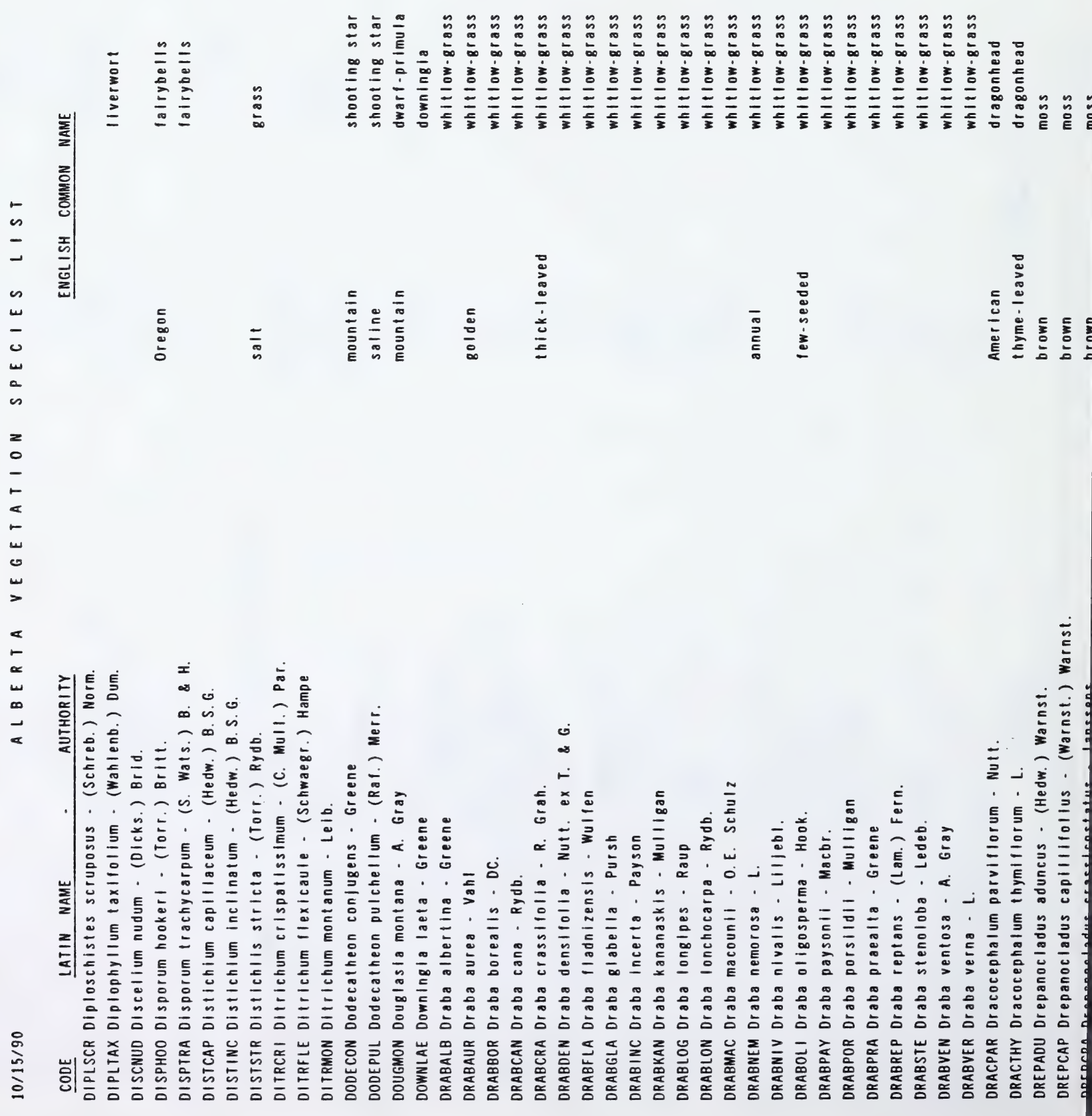




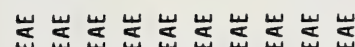

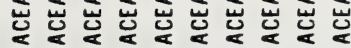

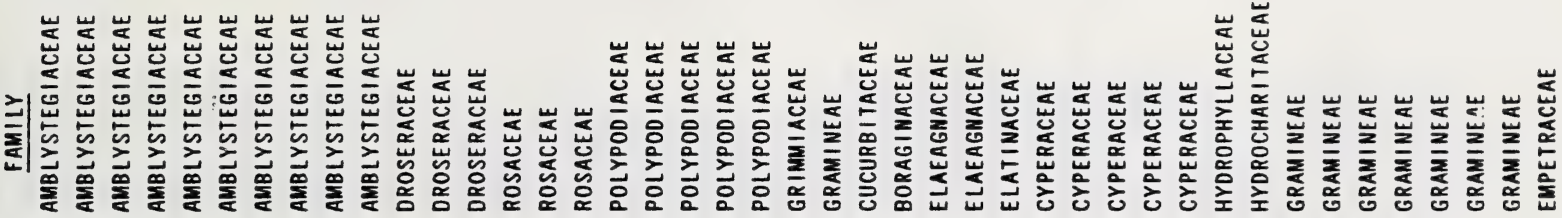

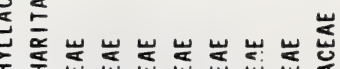
额

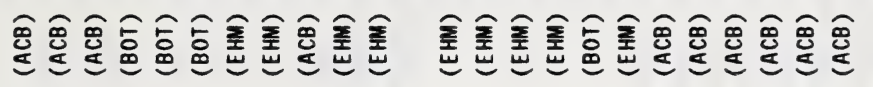

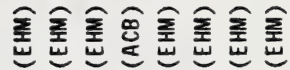
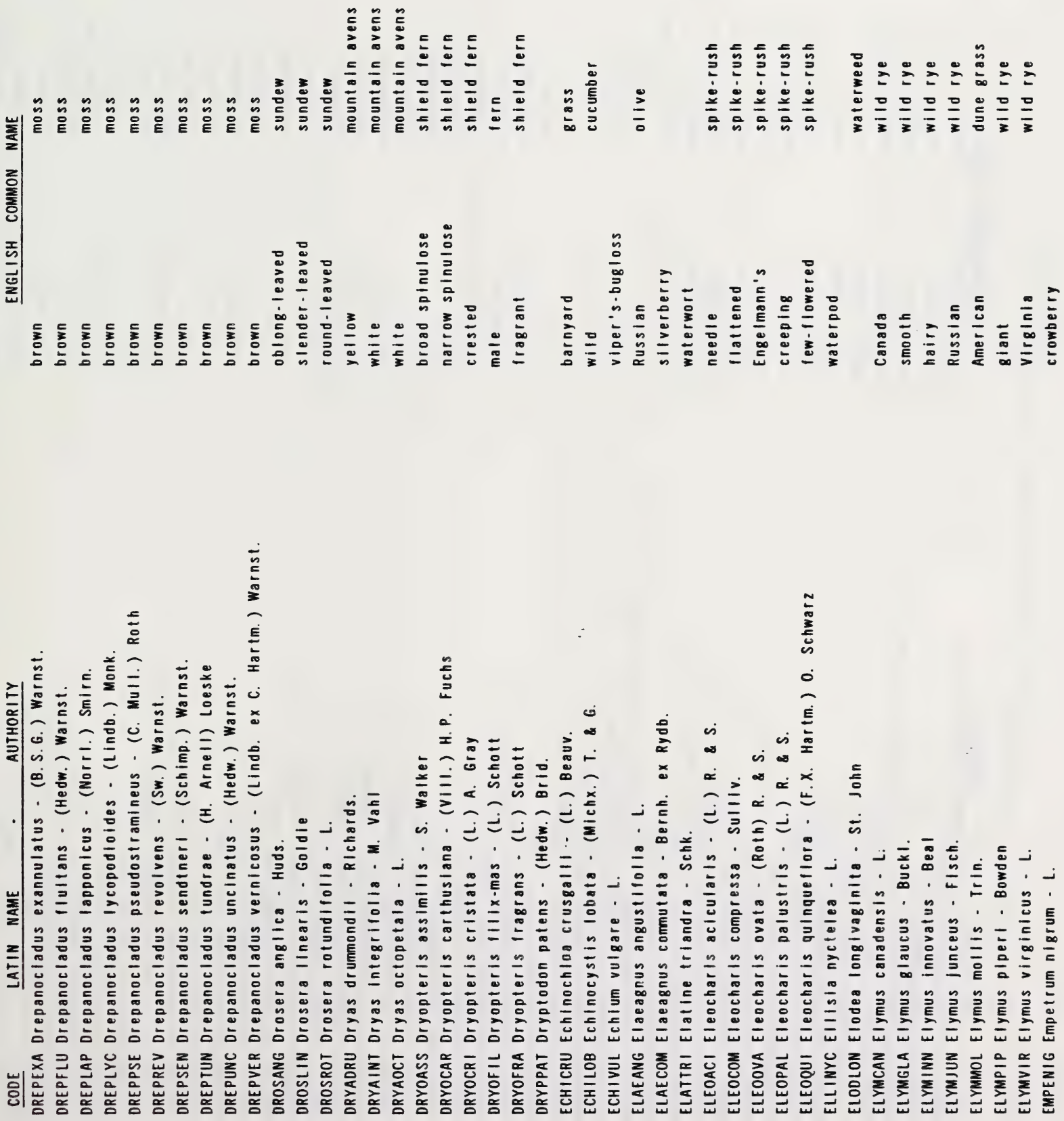


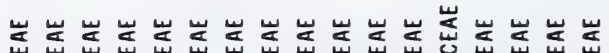

引|

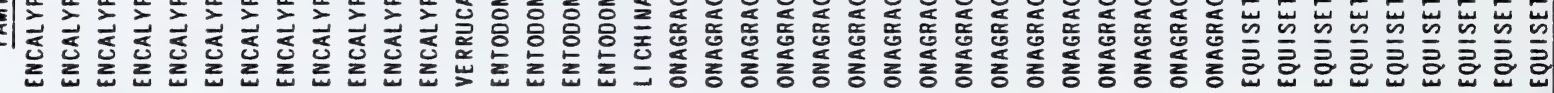

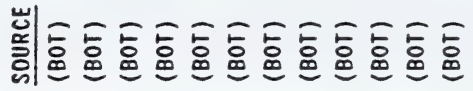

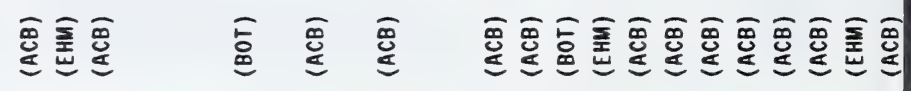
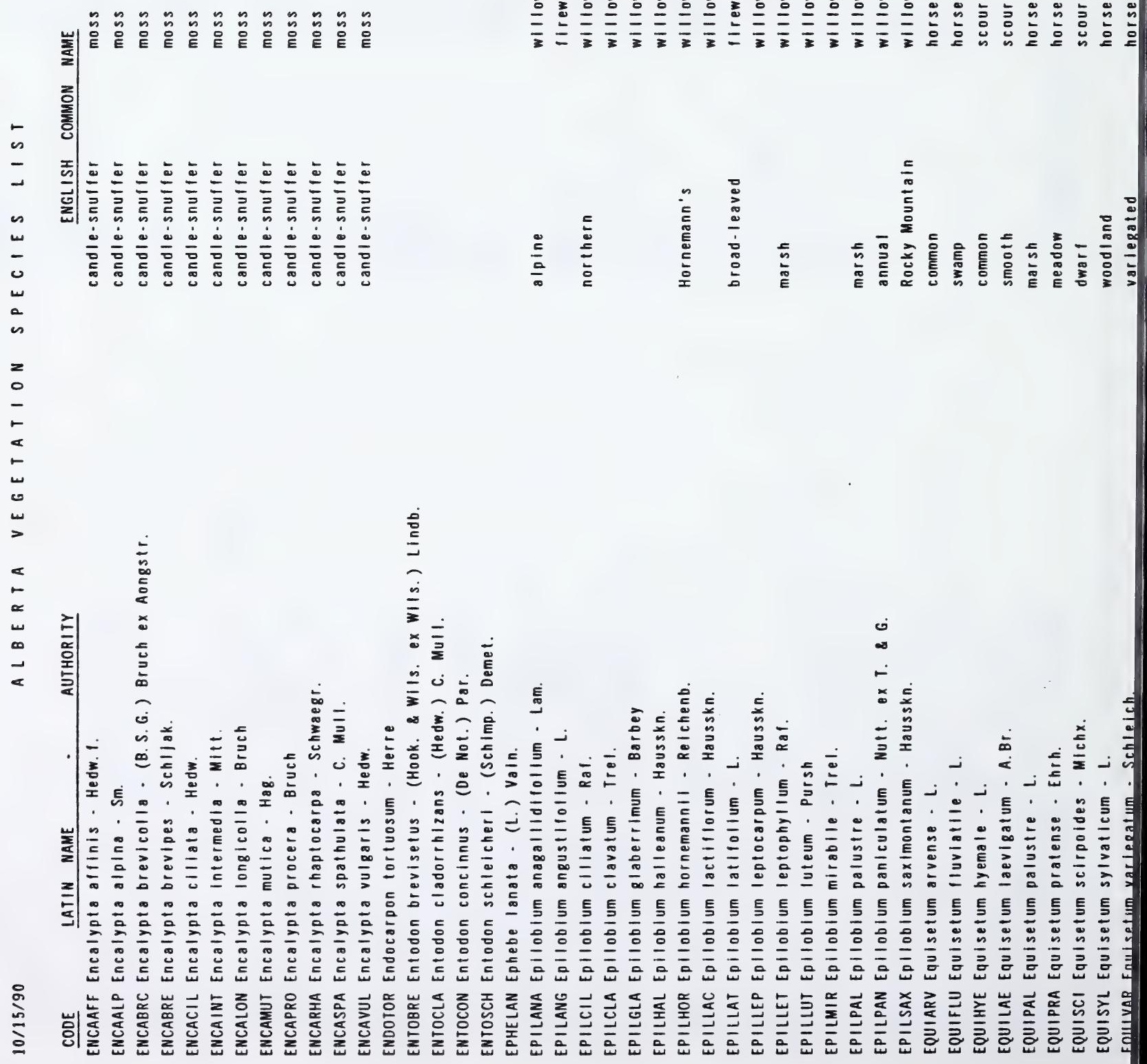


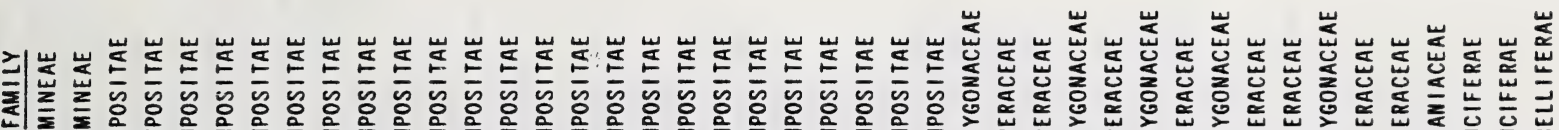

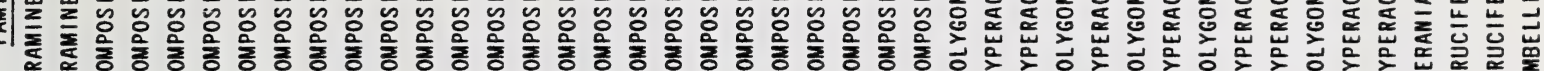

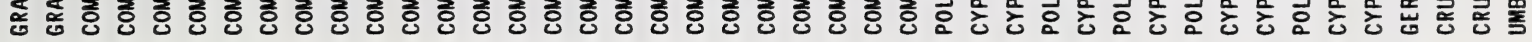

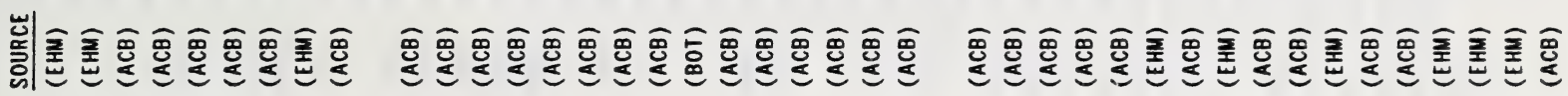

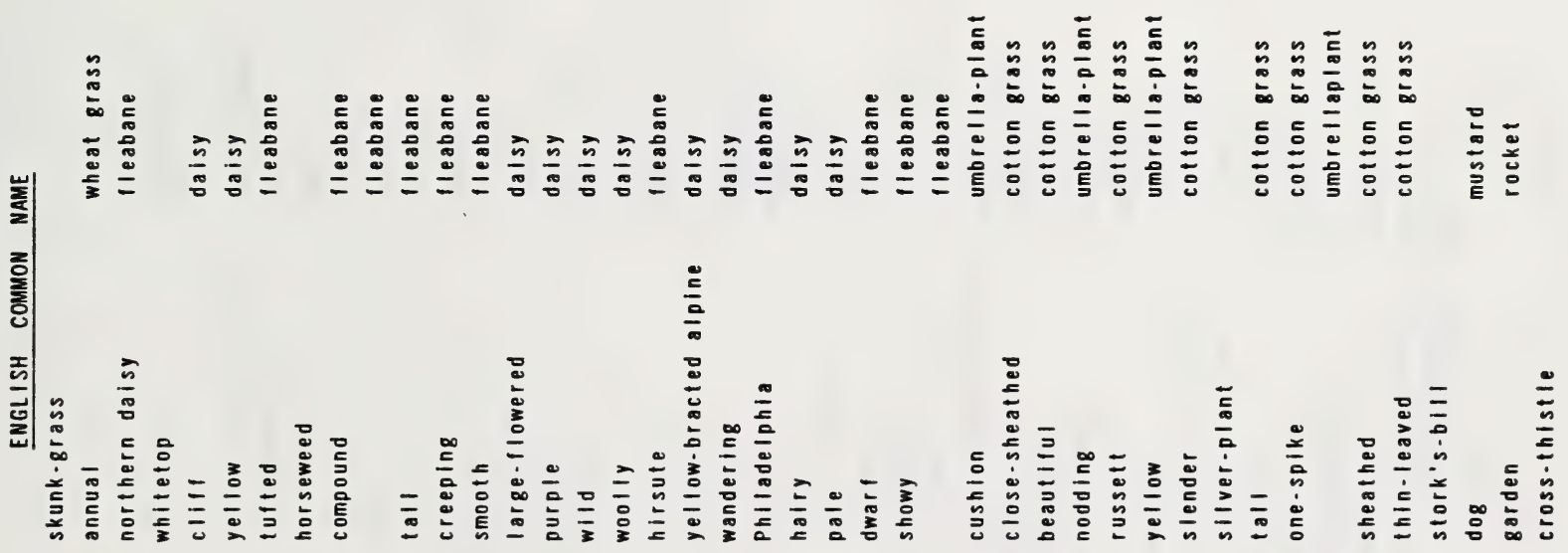

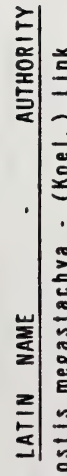
$\frac{1}{2}$ 


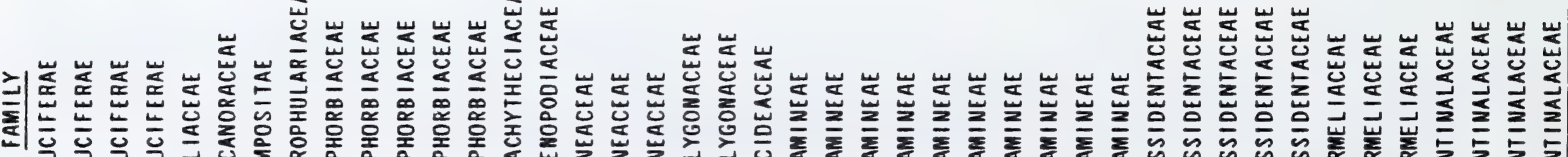

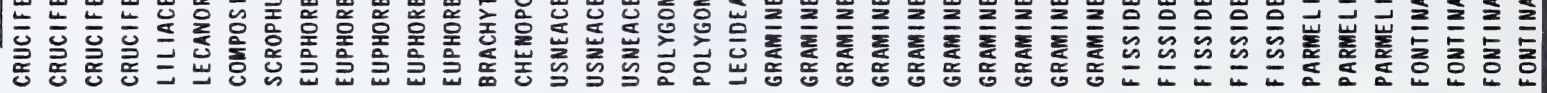

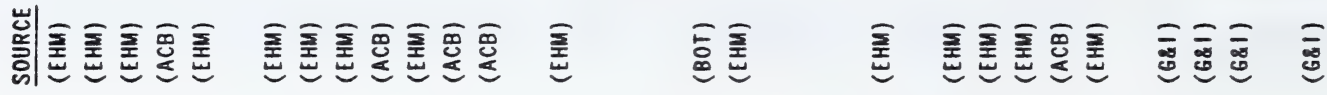
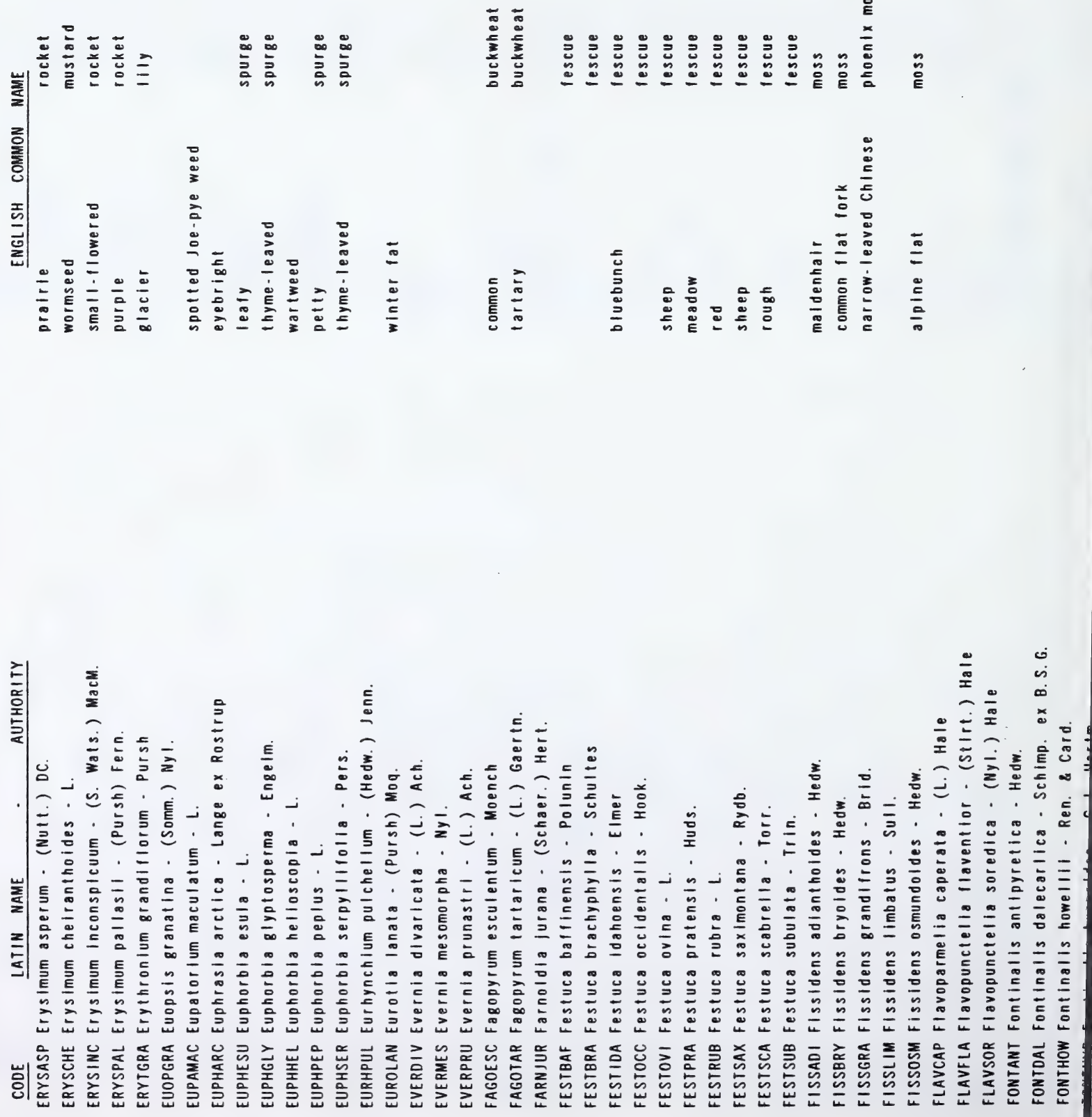


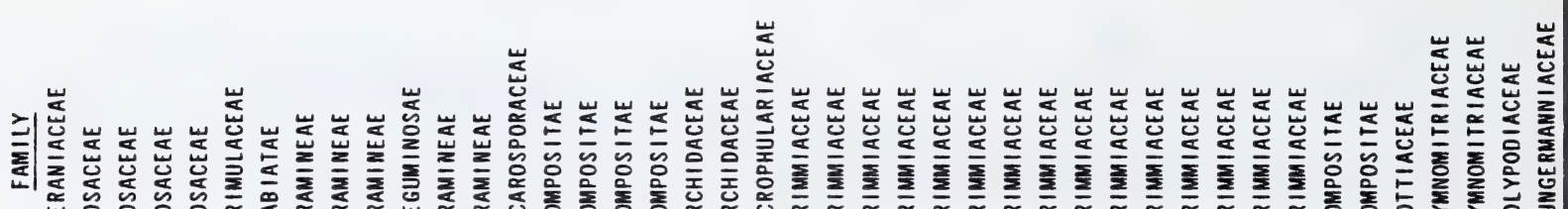

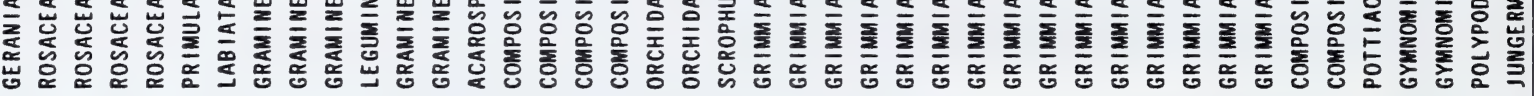

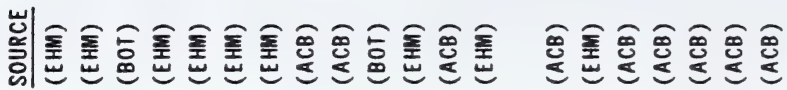

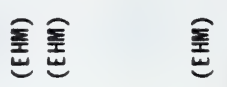

$$
\text { 든 }
$$

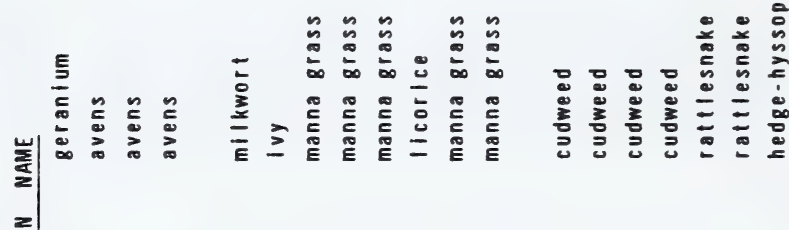

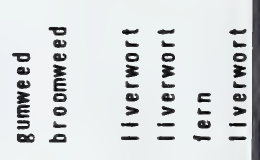

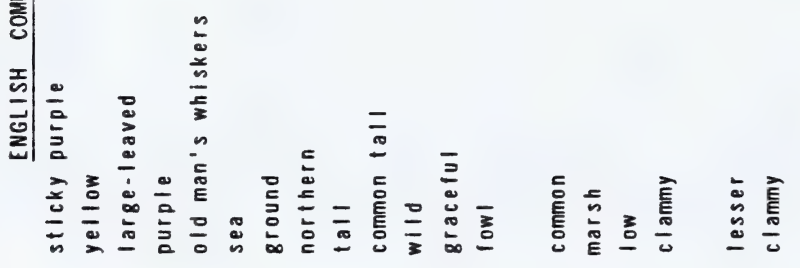


崖㞺

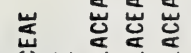

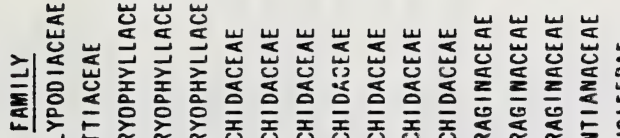

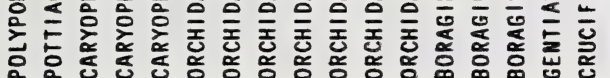

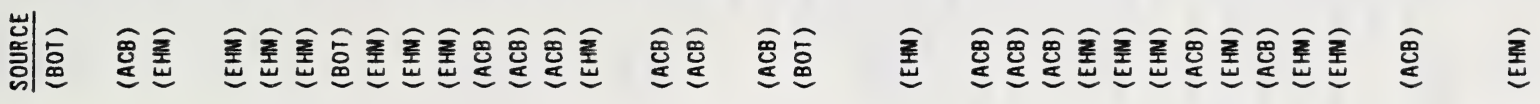

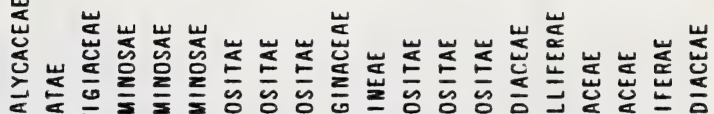

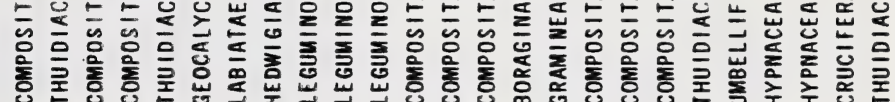

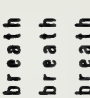

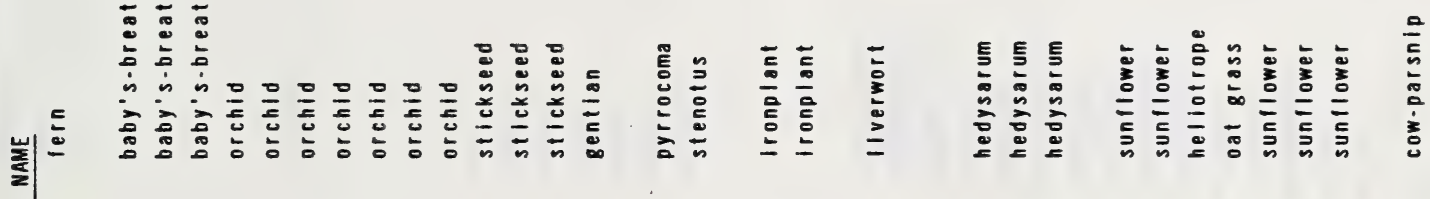

힝. $\stackrel{20}{:}$

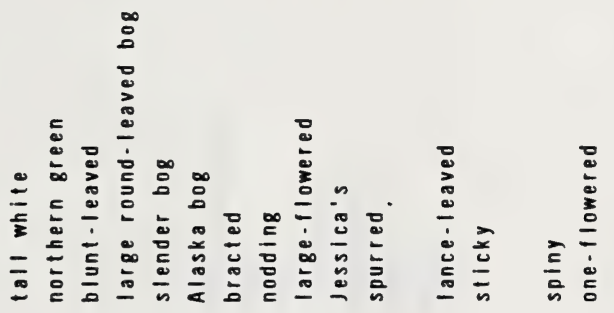

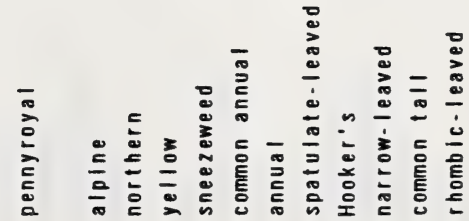

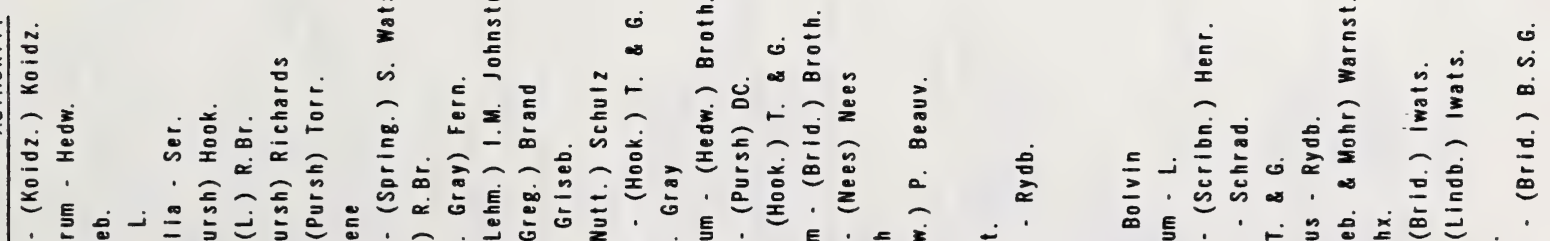

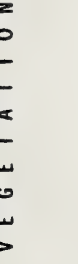

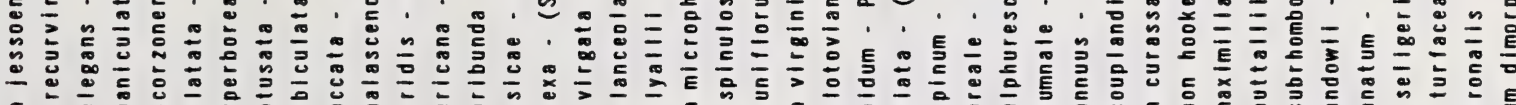

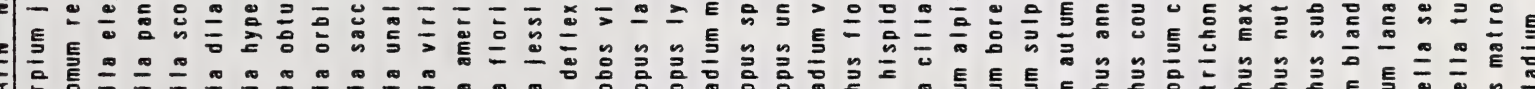

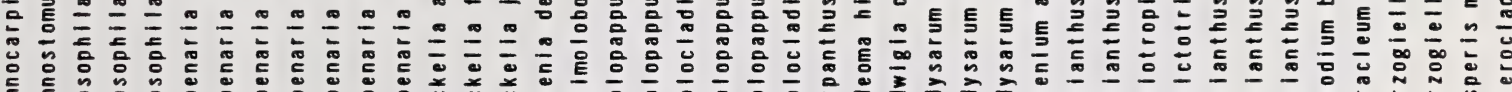

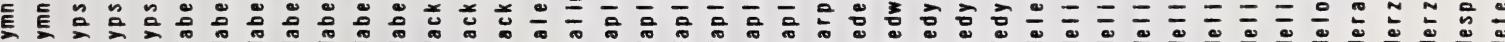

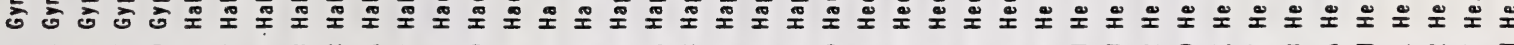

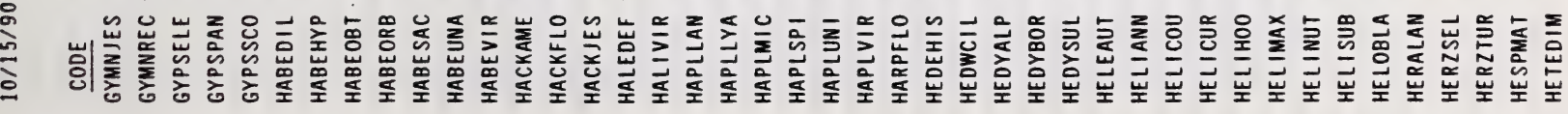




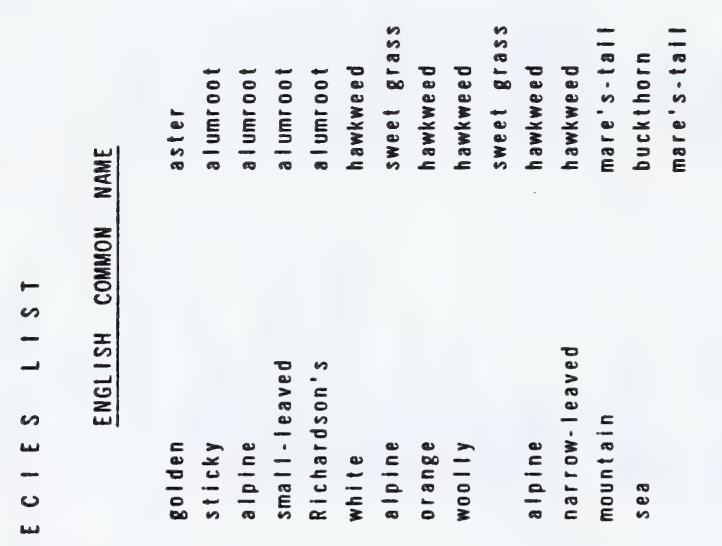




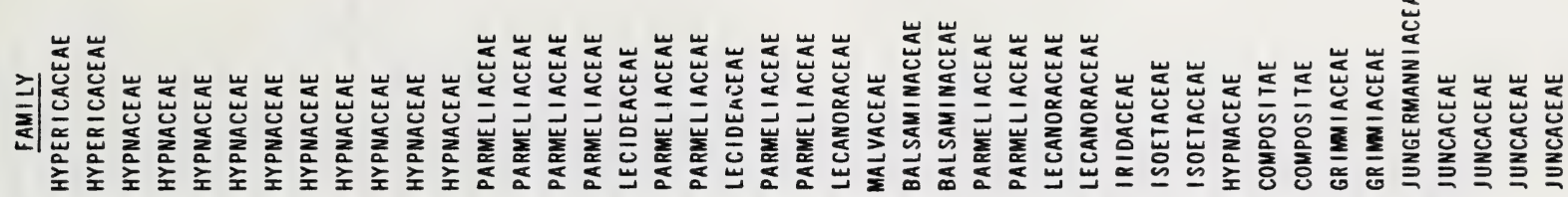

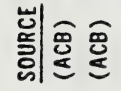

寒害昜

总䍃豆

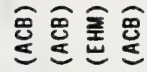
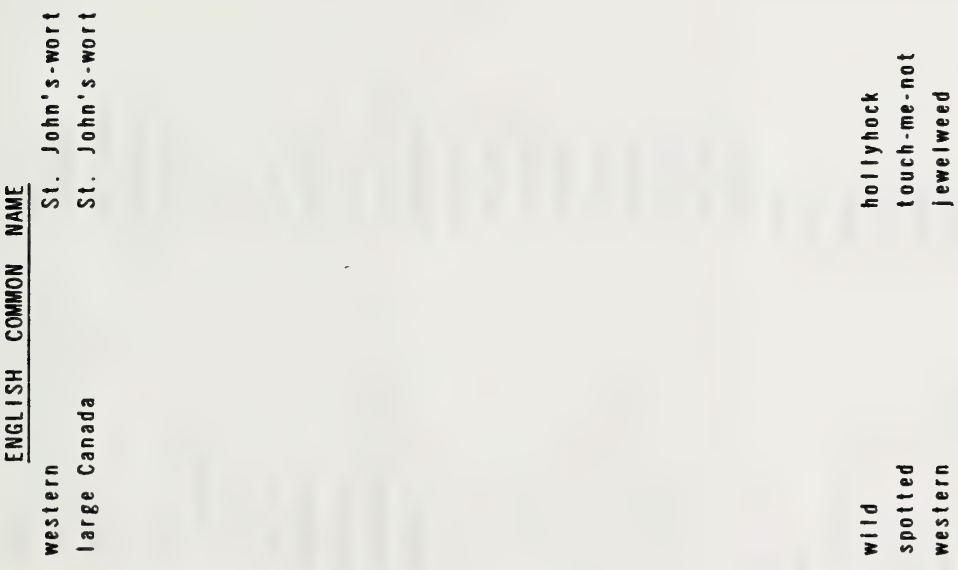

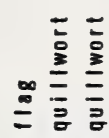

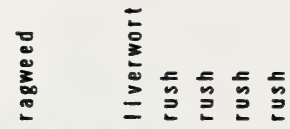

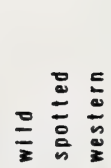

$\sum_{\substack{\vdots \\ \vdots}}^{\vdots}$

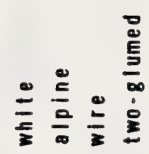

름

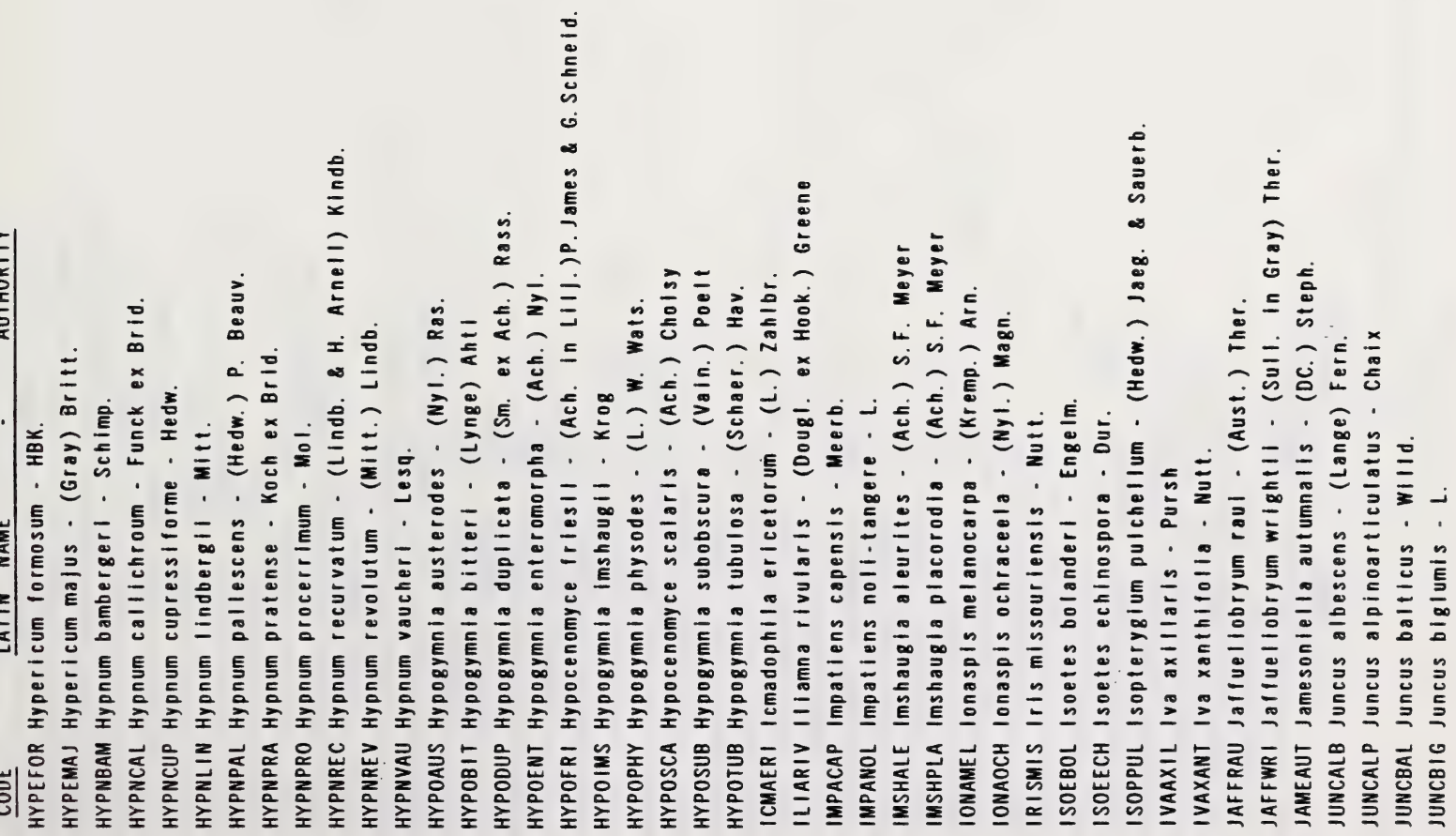




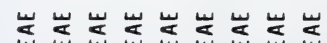

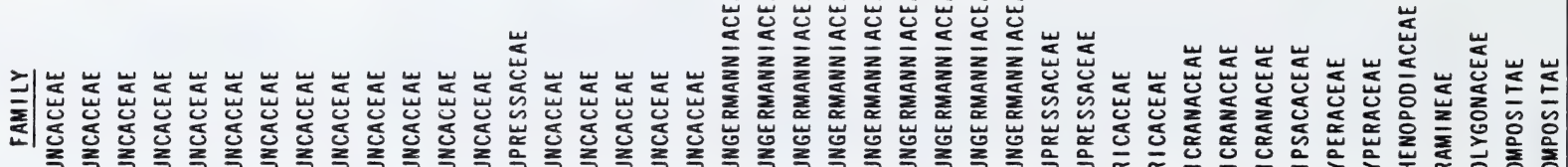

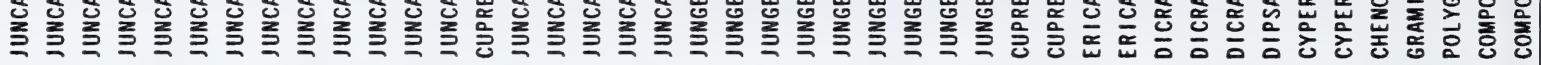

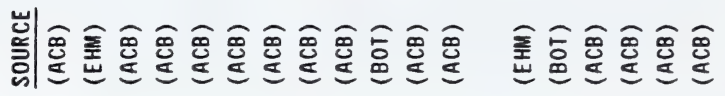

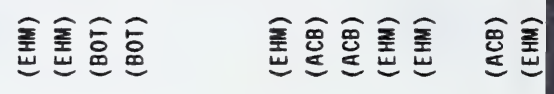

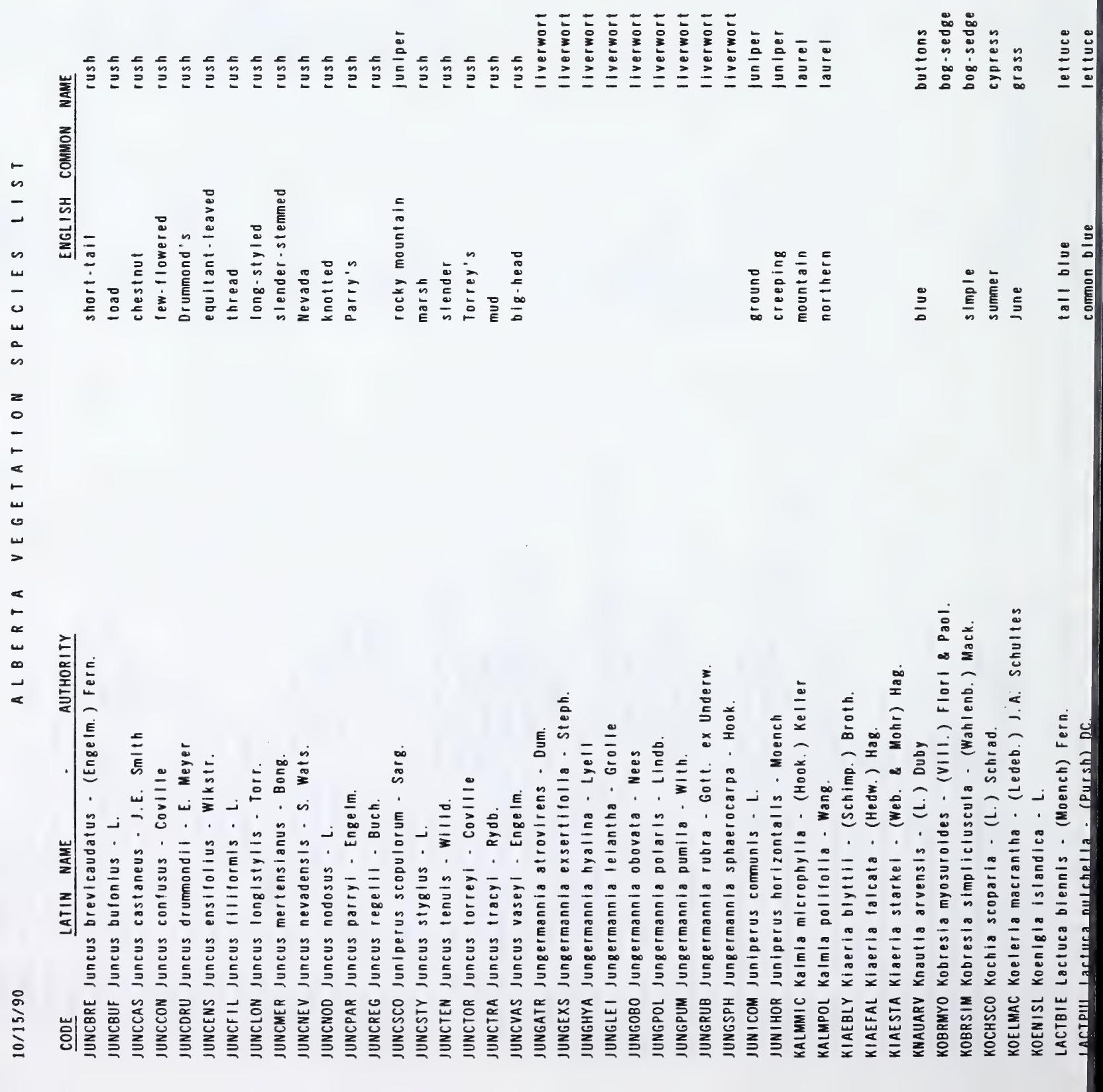




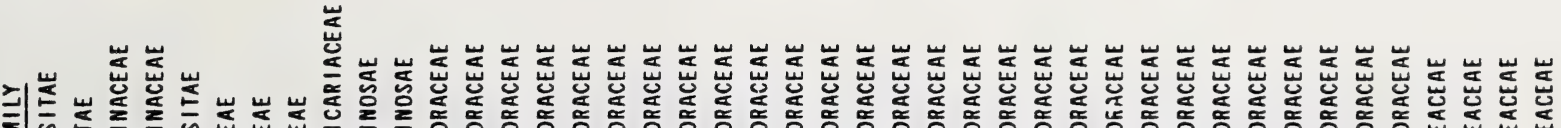

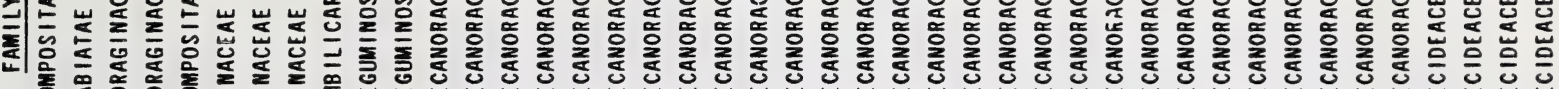

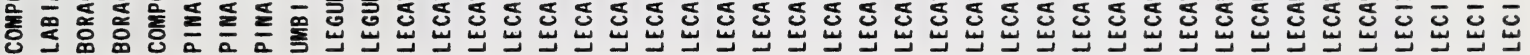

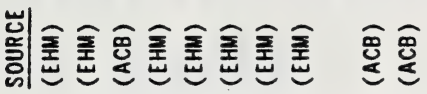
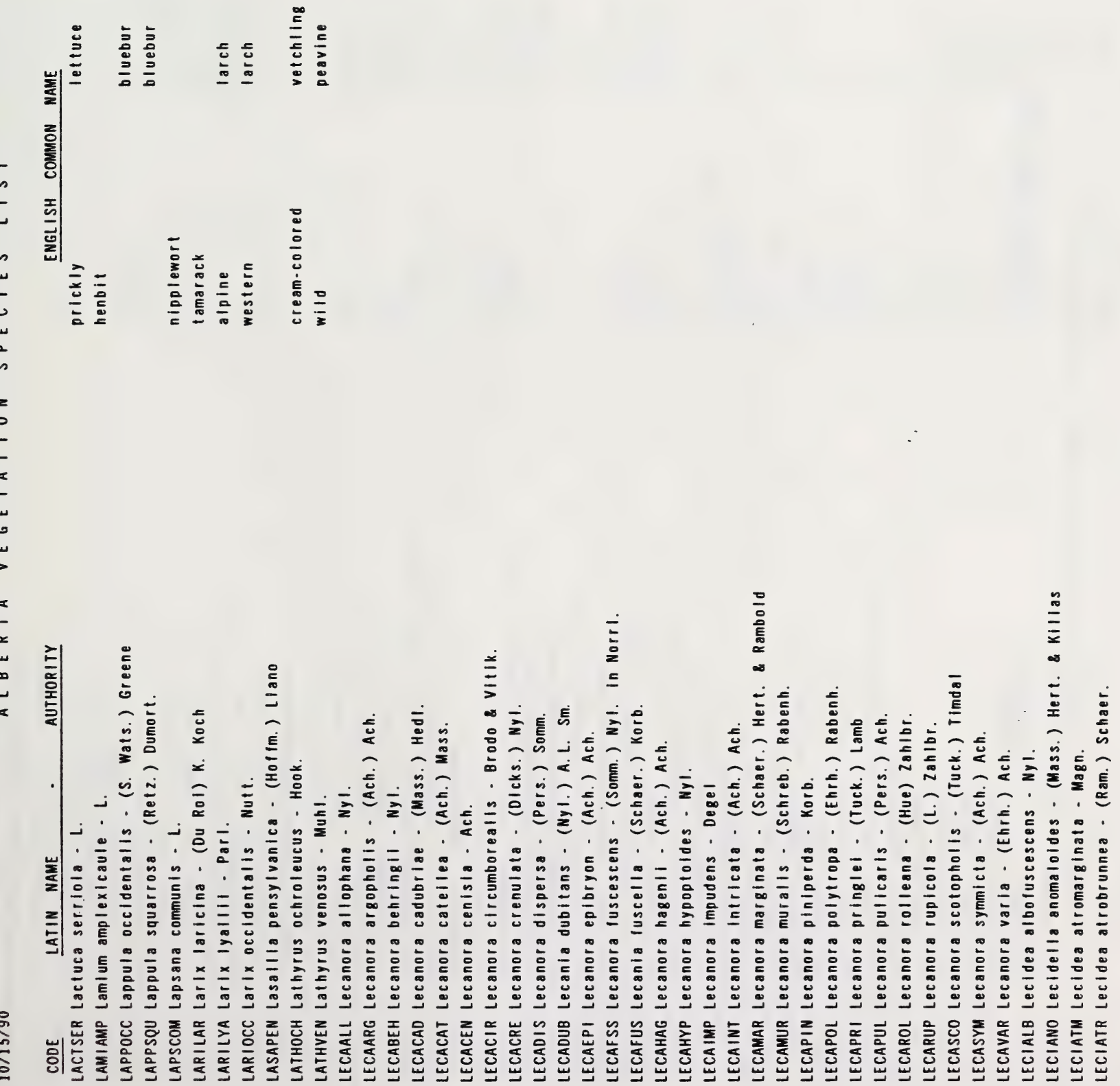


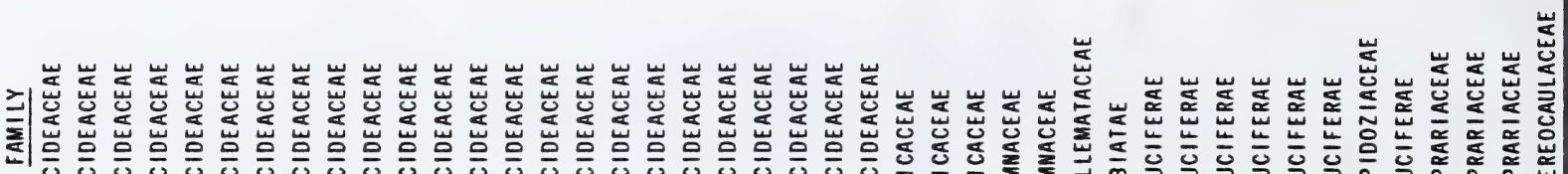

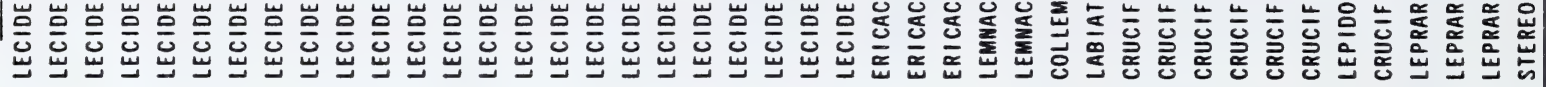
訔|

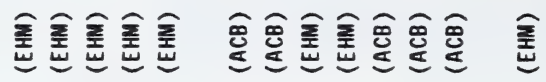

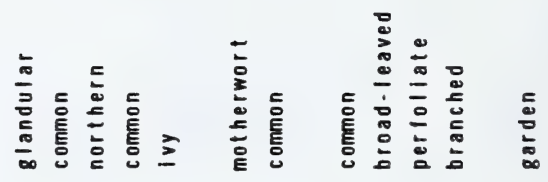

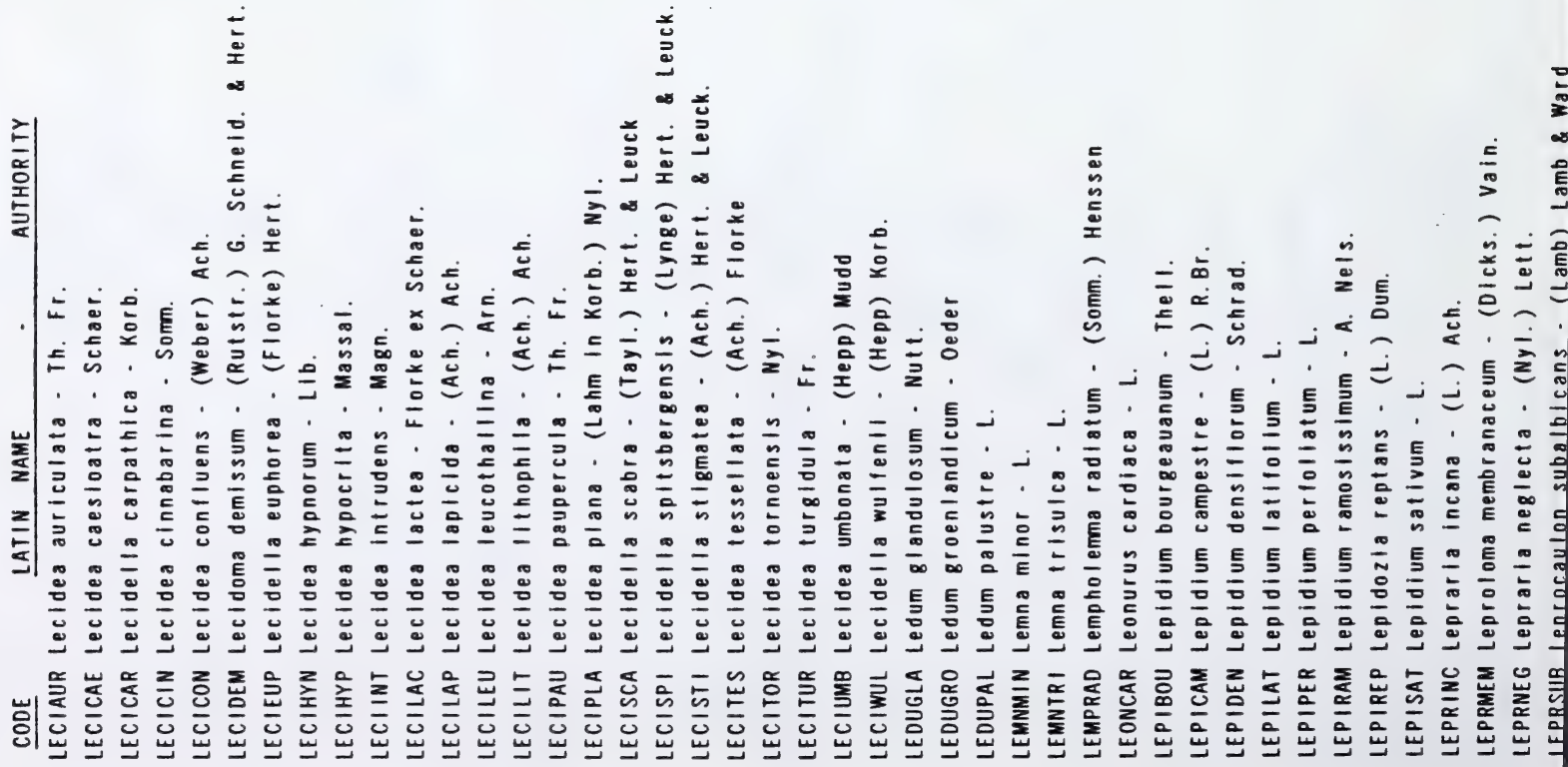




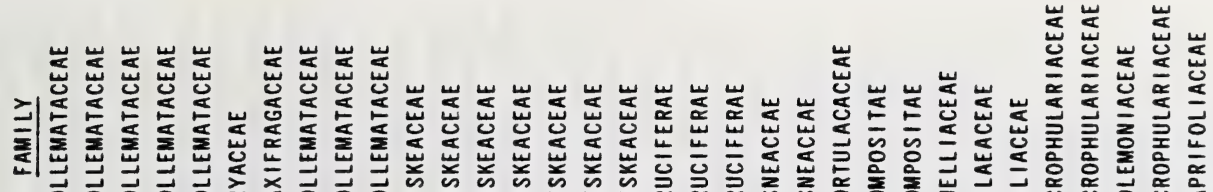

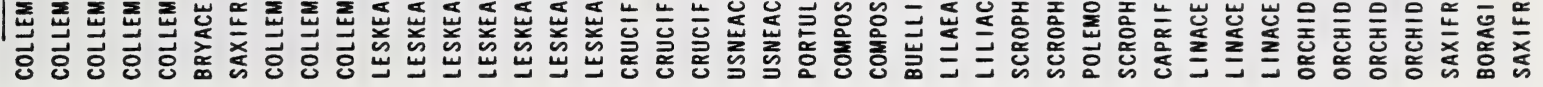
岕|

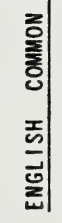
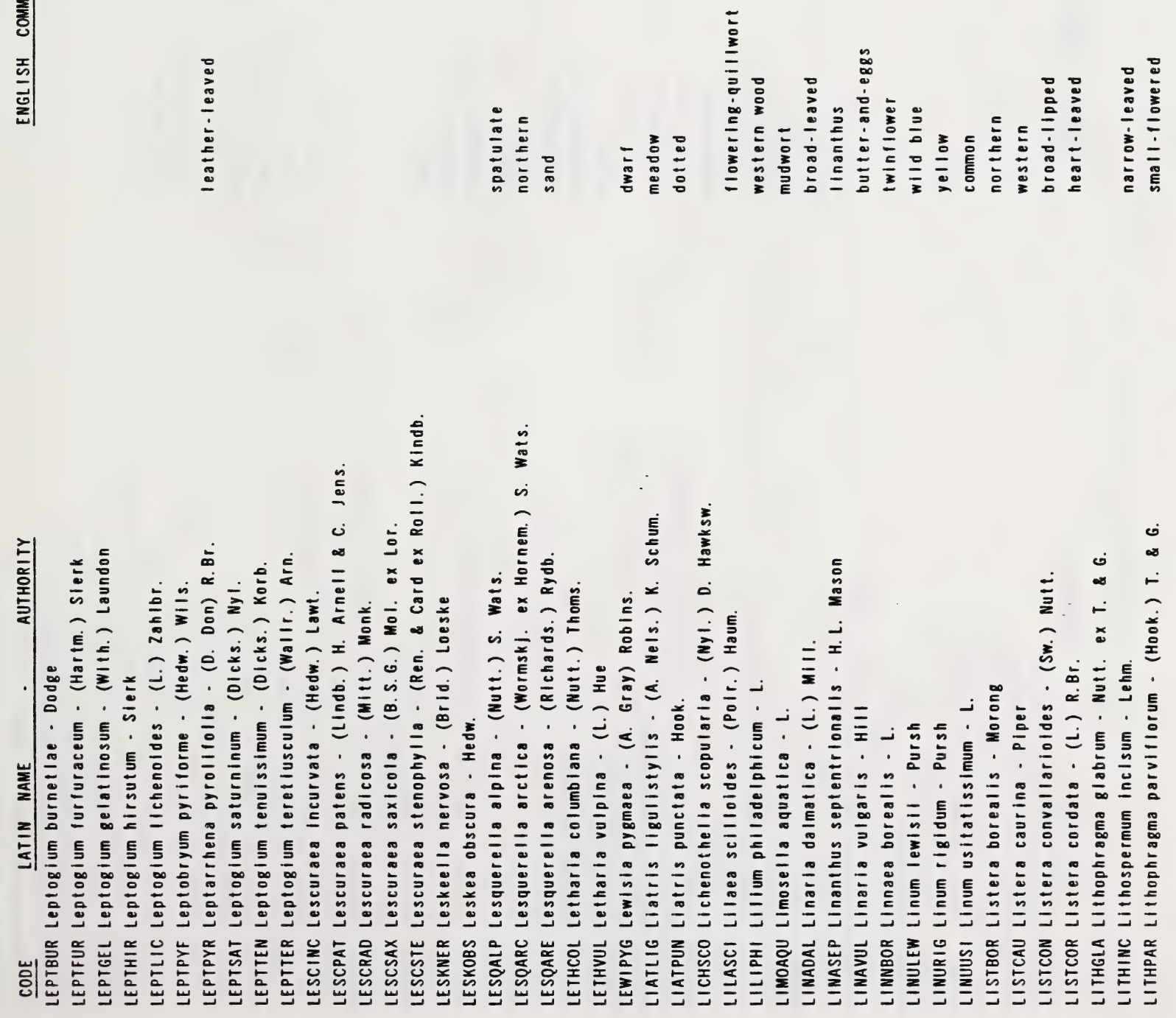


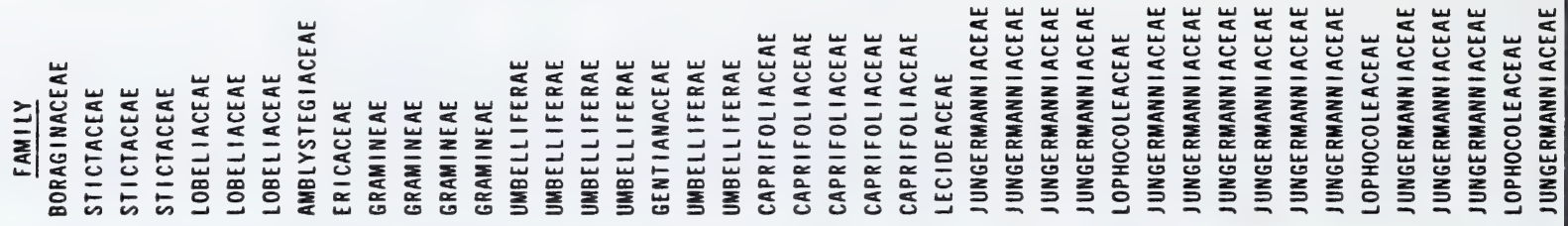

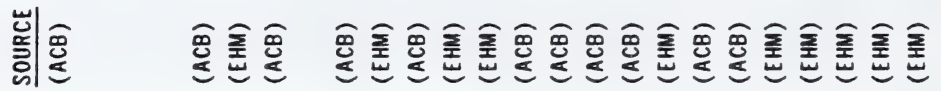

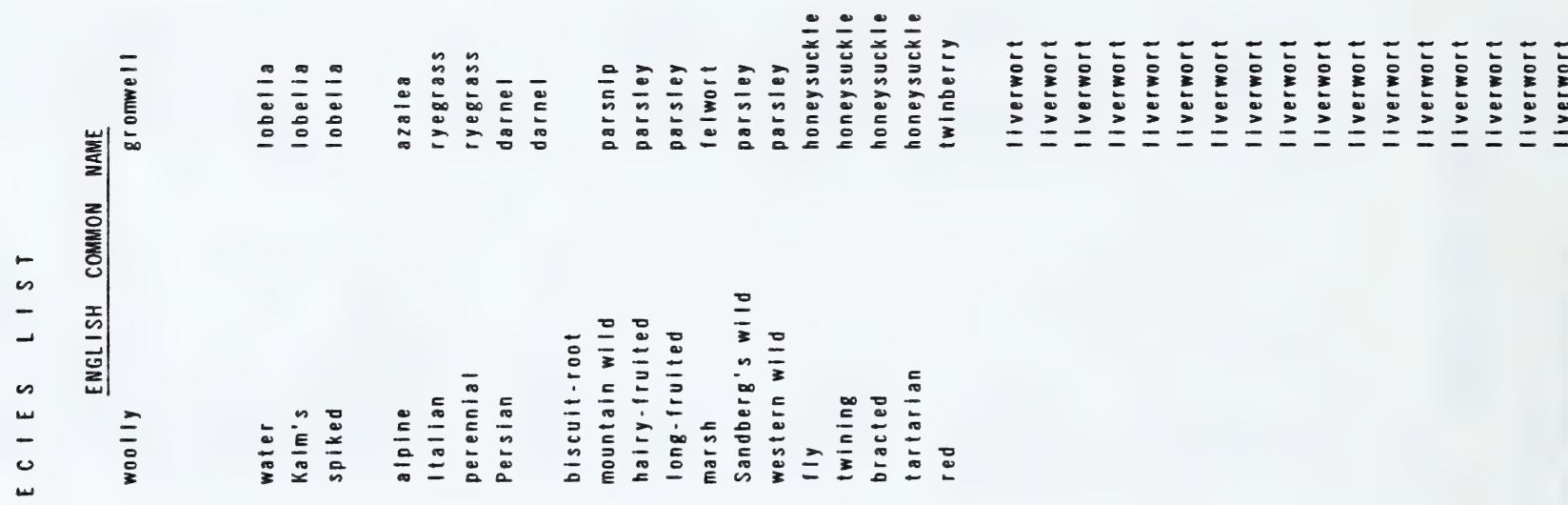

$<$
$\infty$
$\infty$
$\infty$
$\infty$

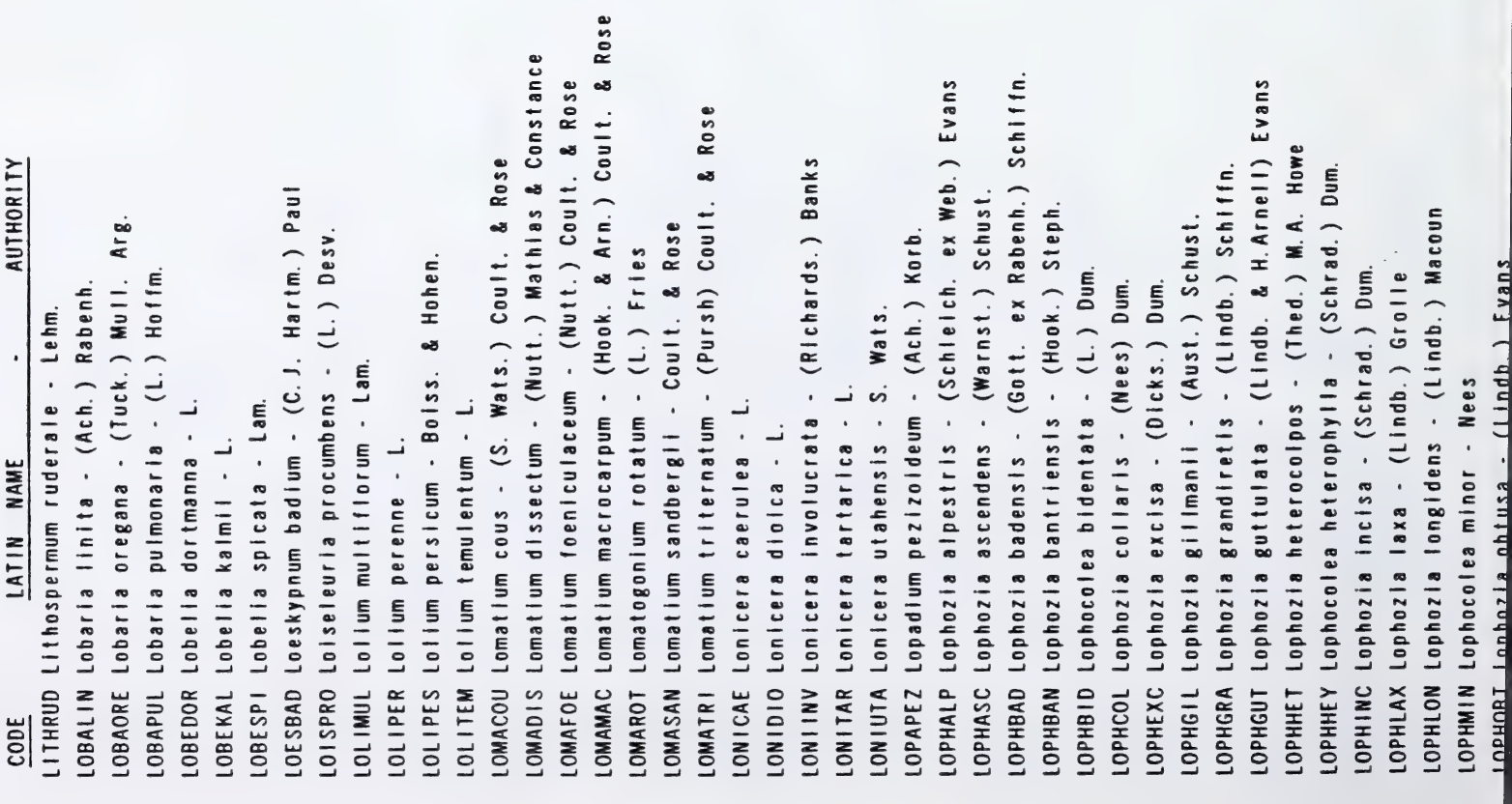


崖岕岕

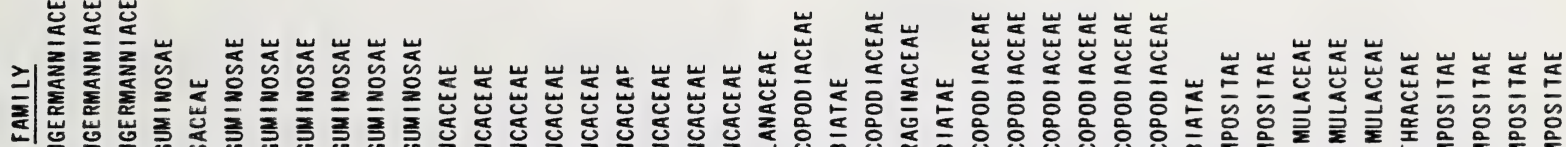

峛

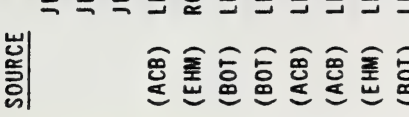

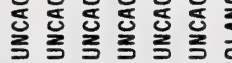

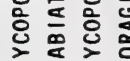

要

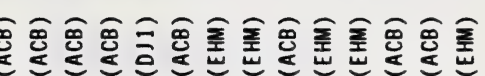

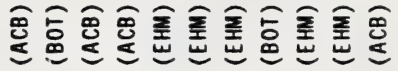

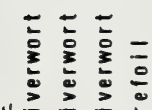

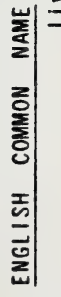

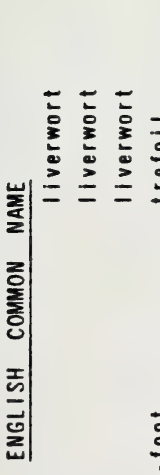

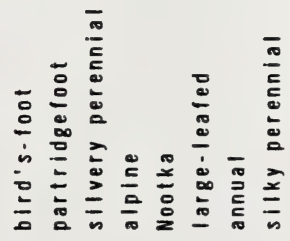

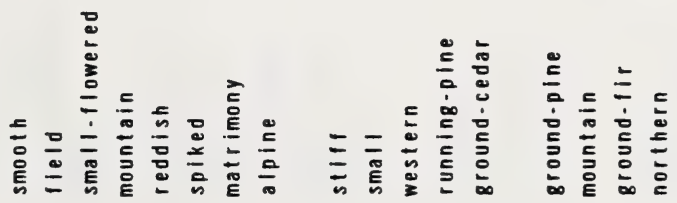

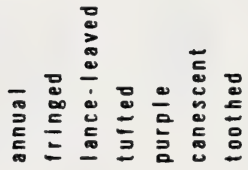

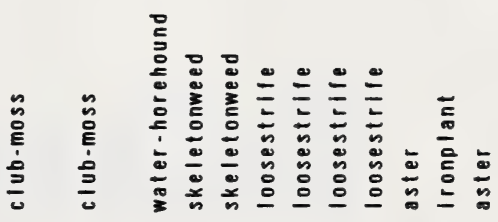

5

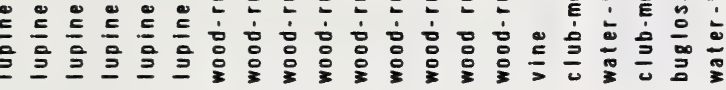

o

a

$z$

0

$\overline{-}$

$\leftarrow$

0

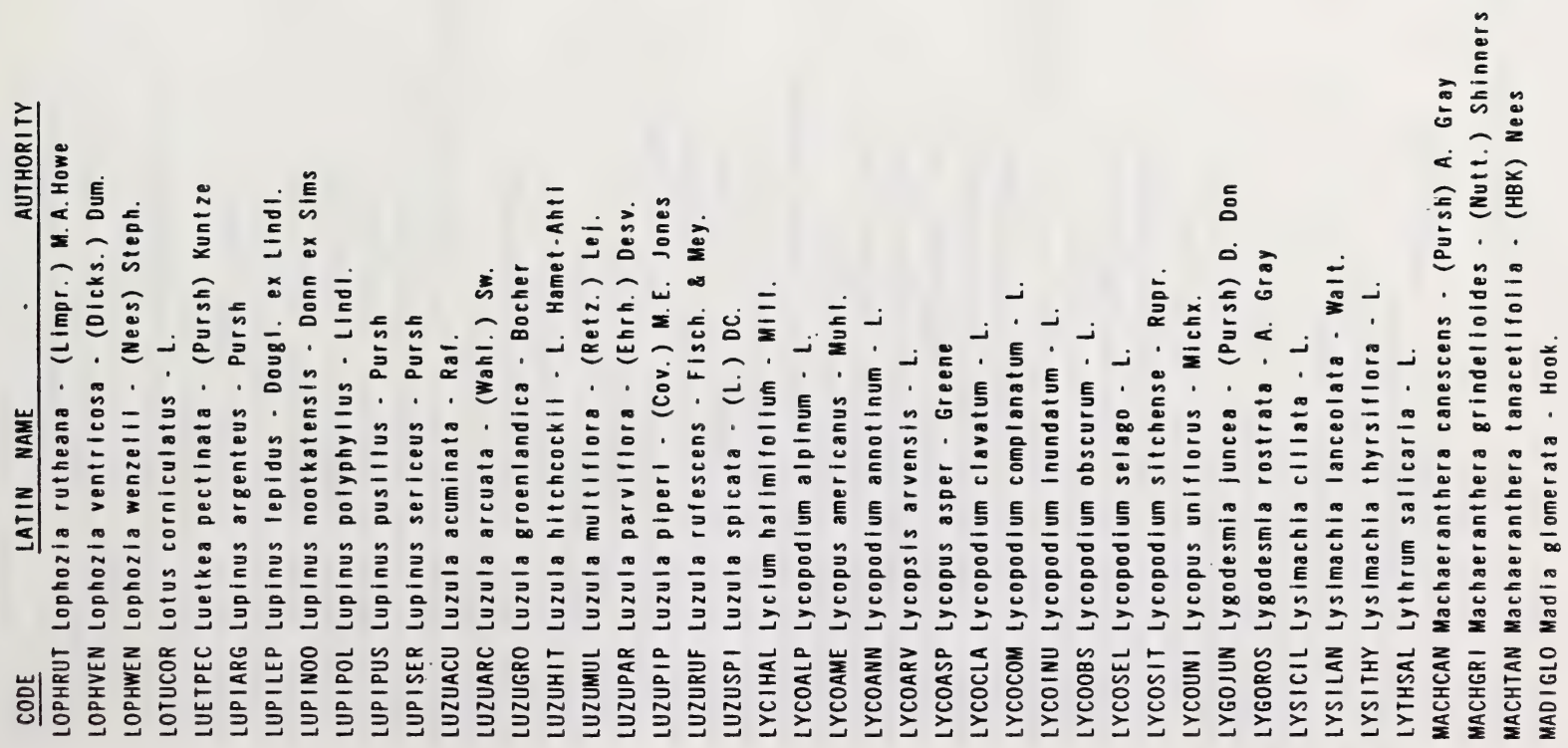




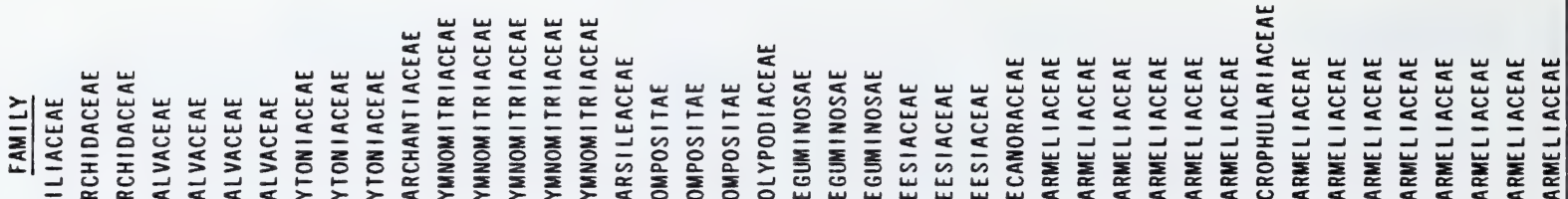
三

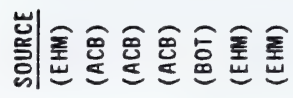
要金金金金恶金恶 重

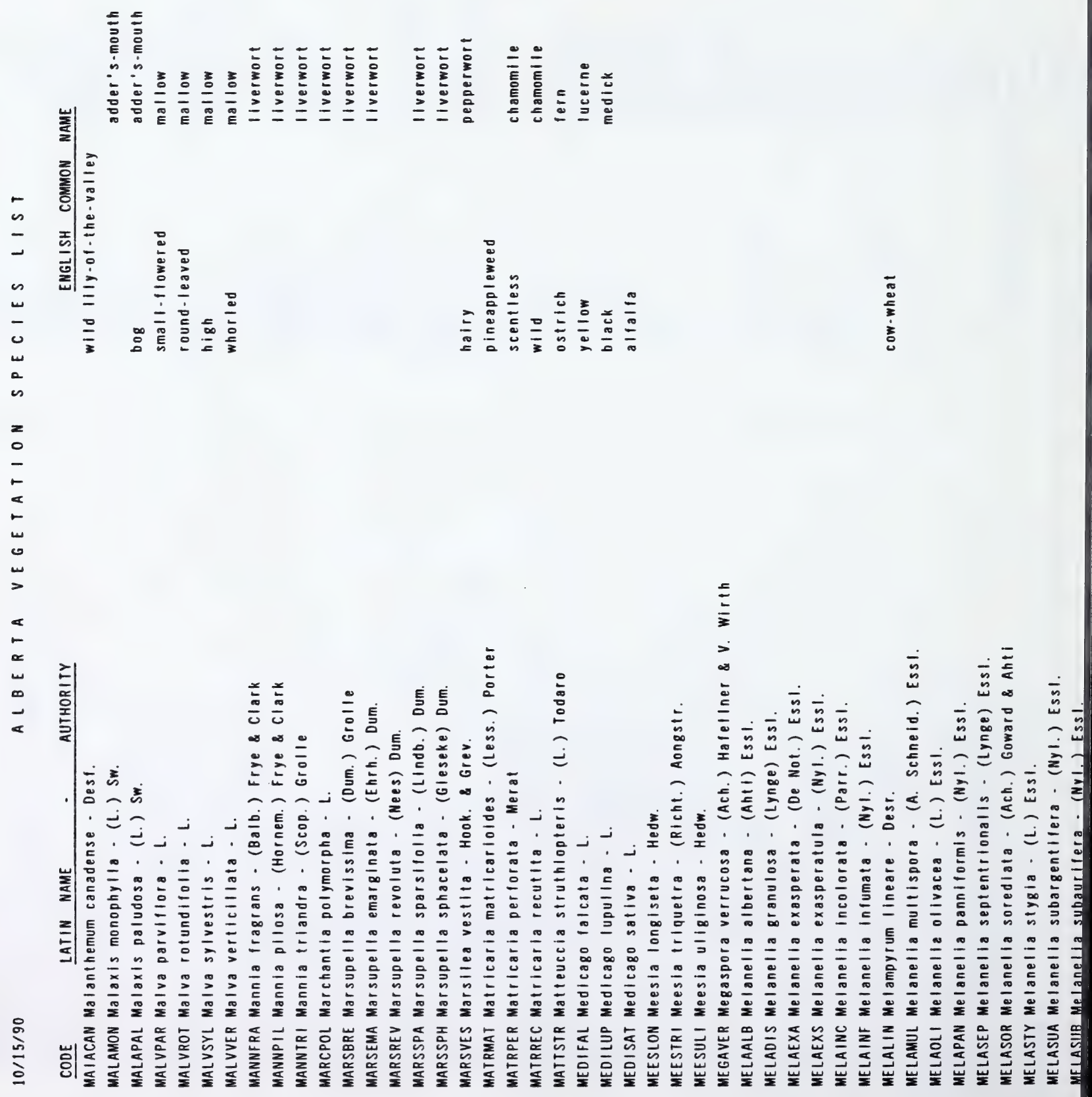




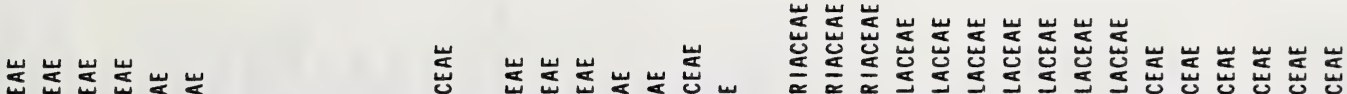

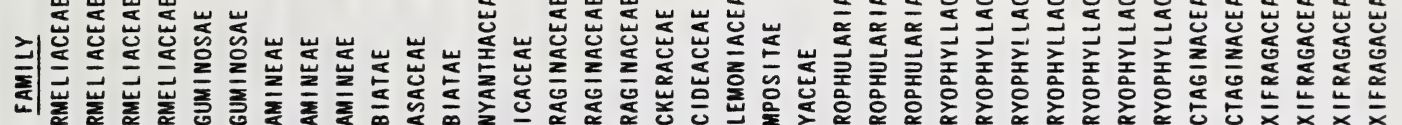

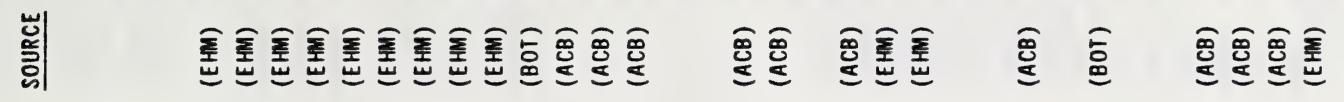

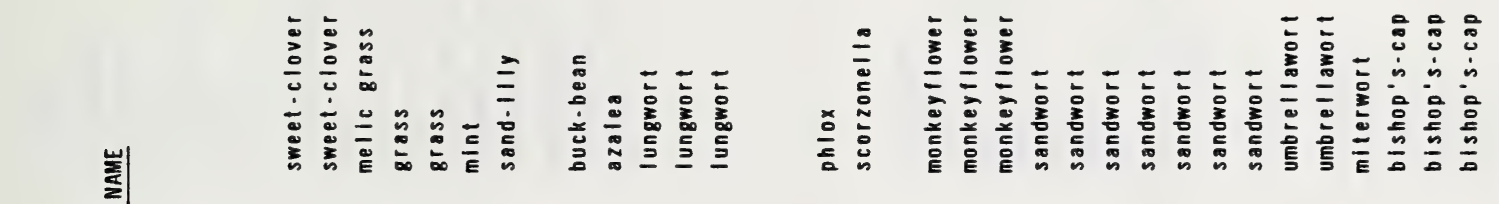

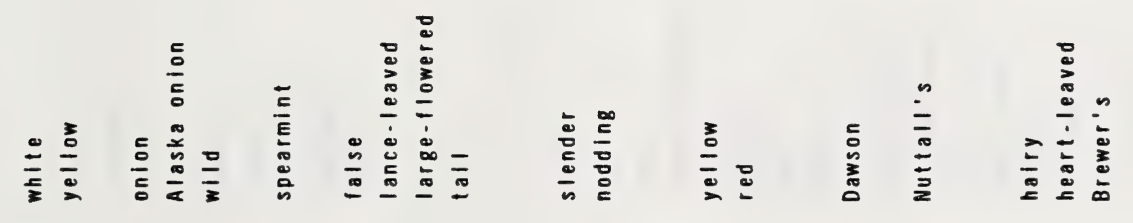

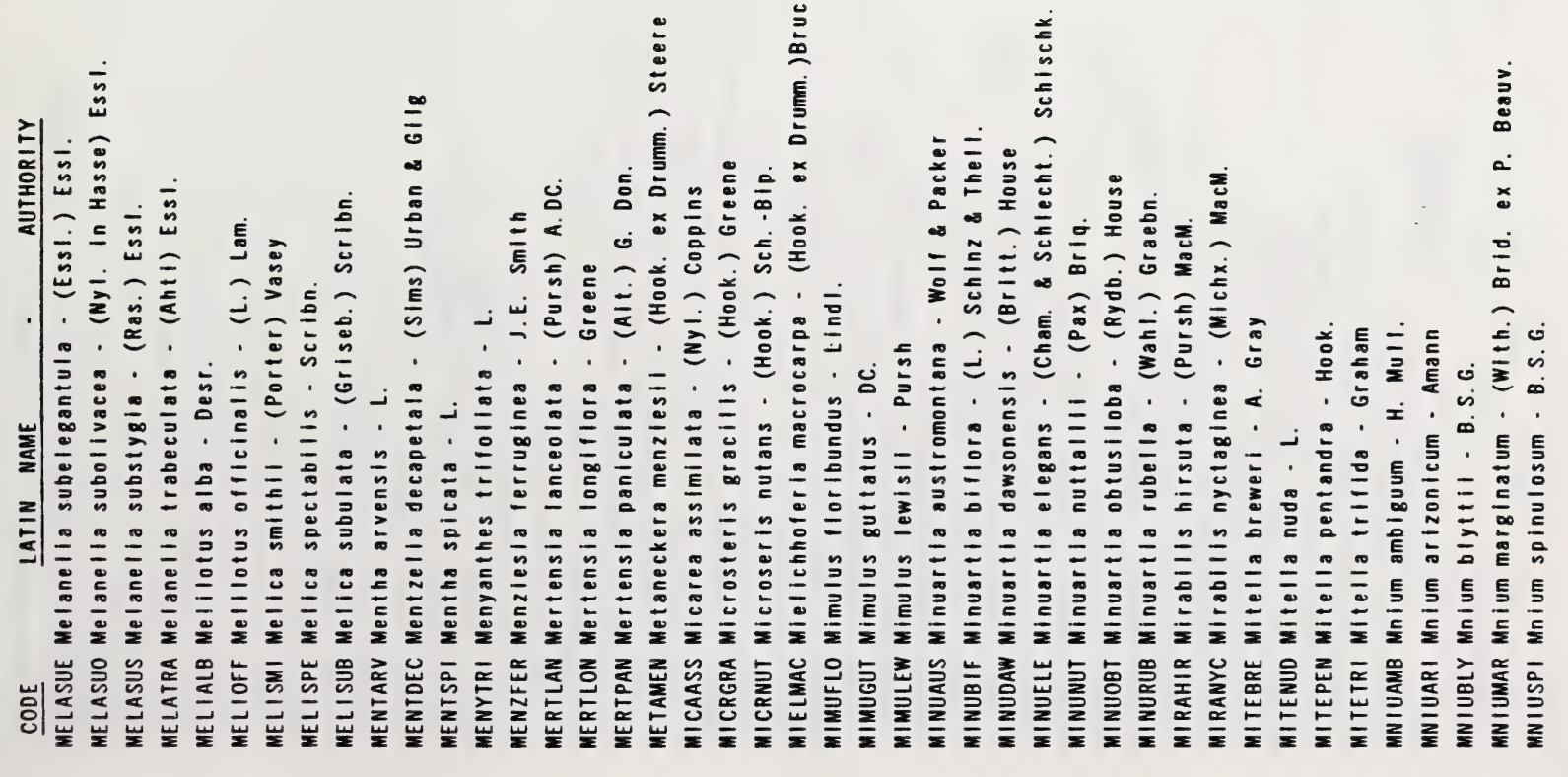




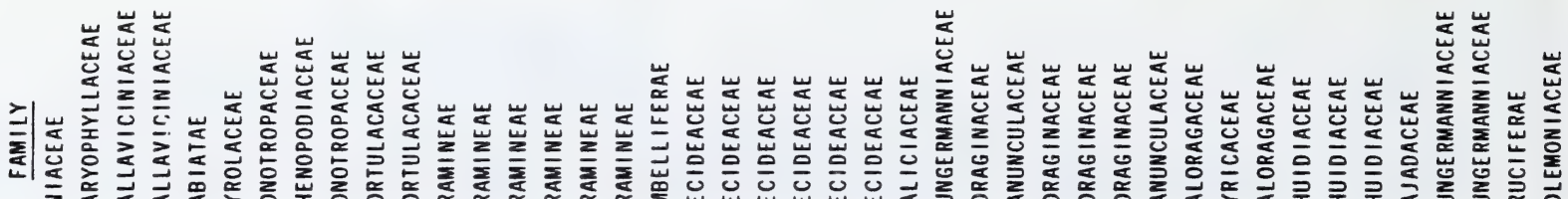

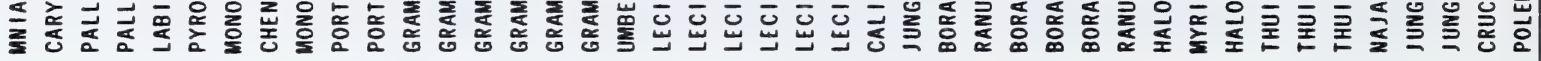

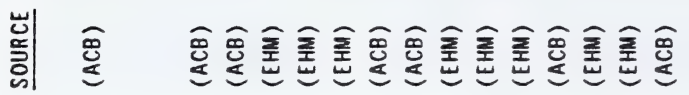

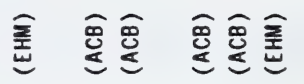

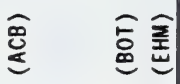
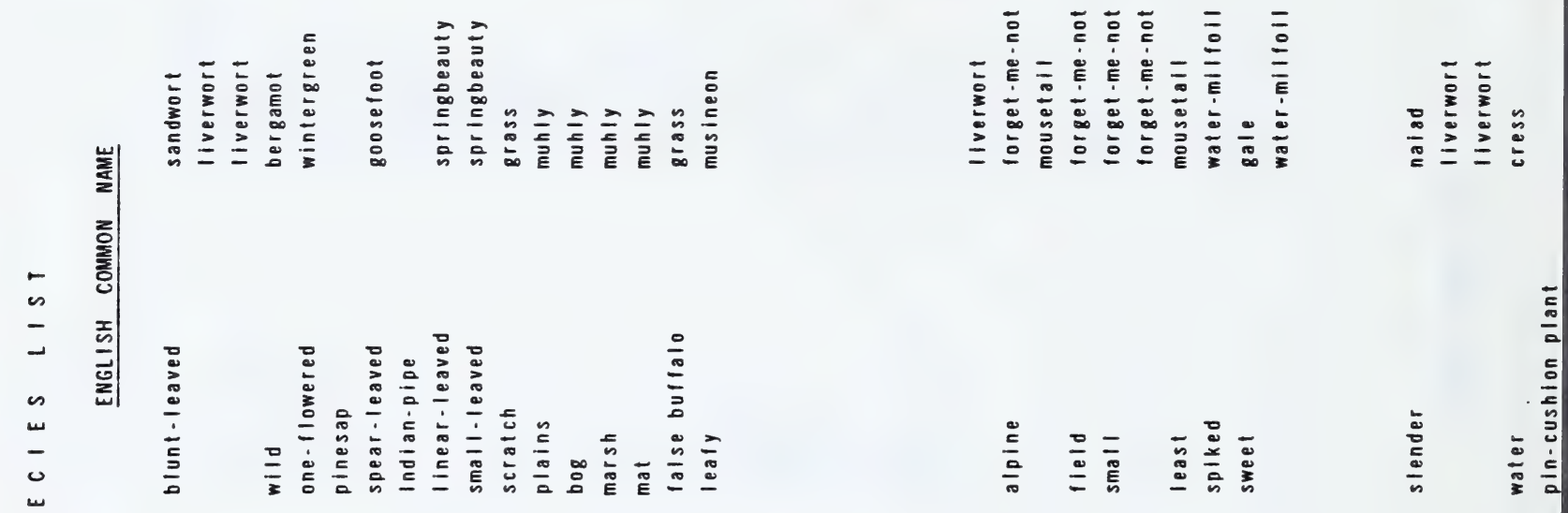

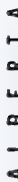

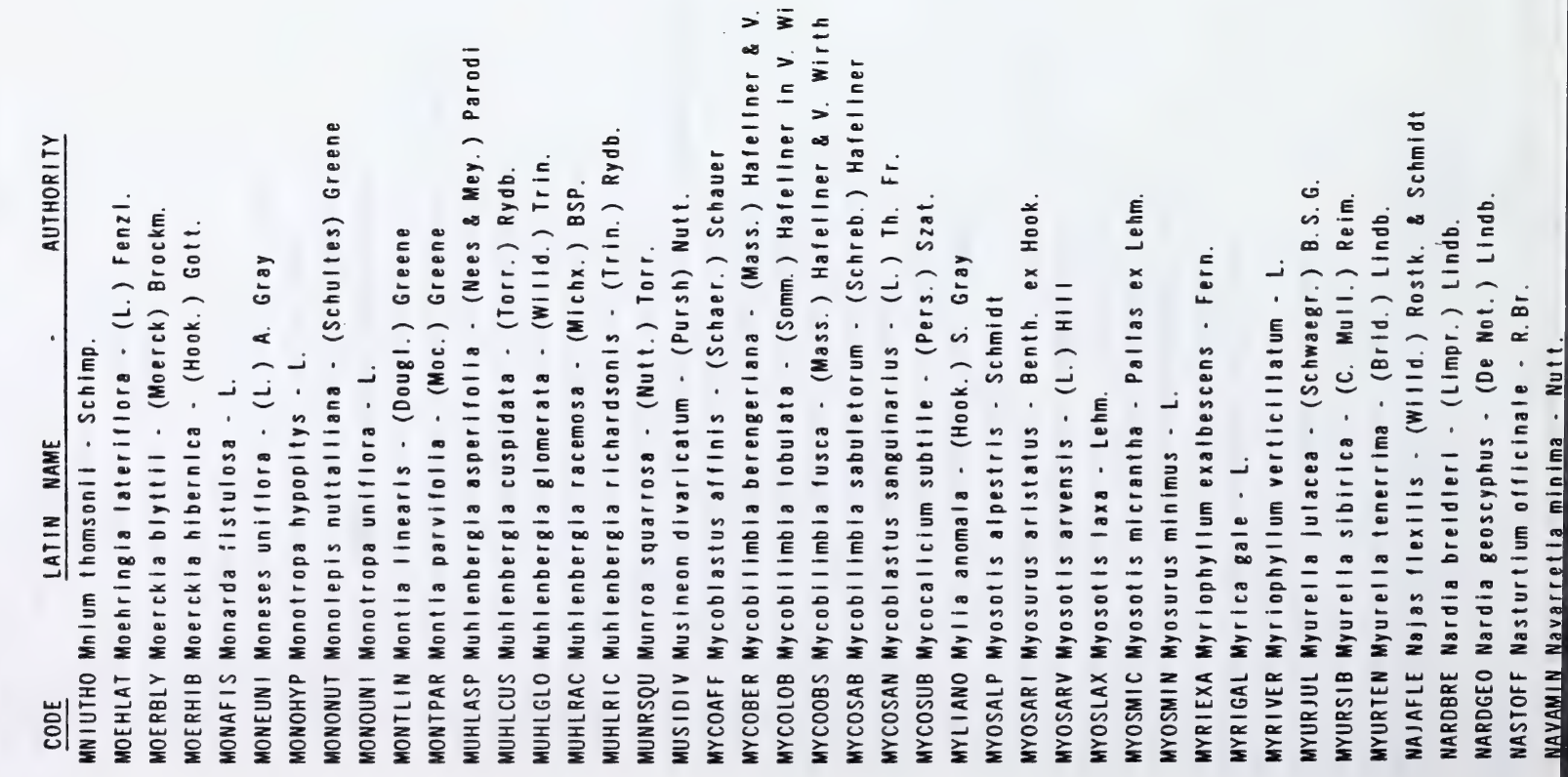




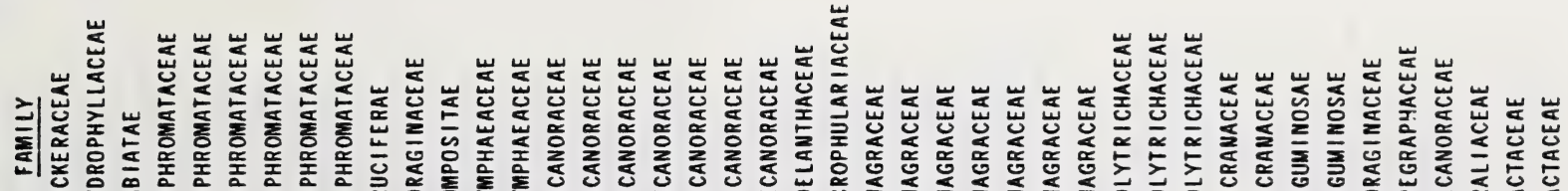

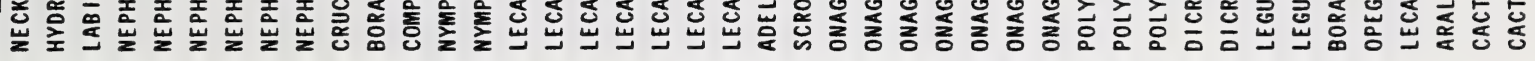

鿷要金

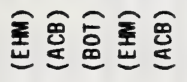

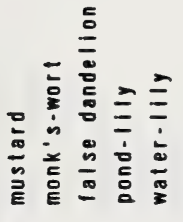

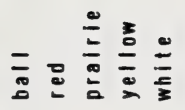

垔

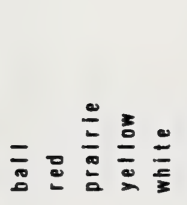

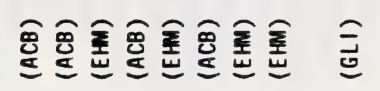

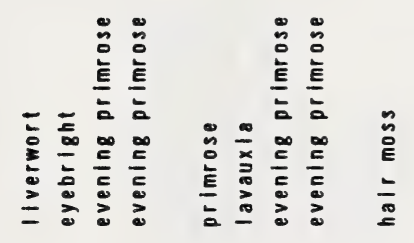

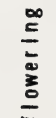

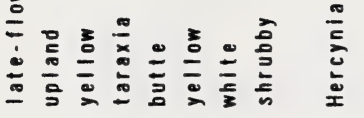

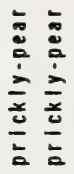

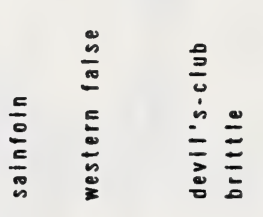

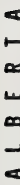

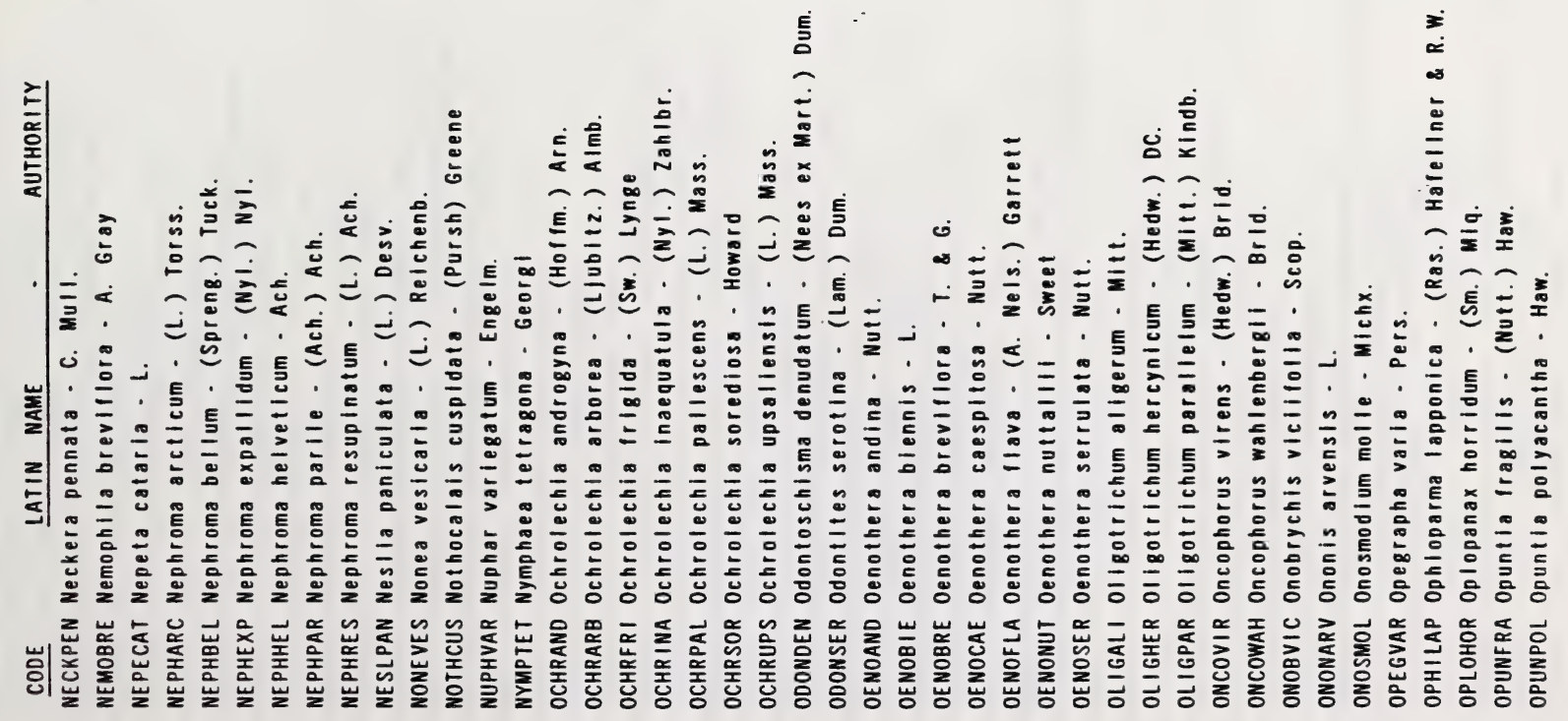




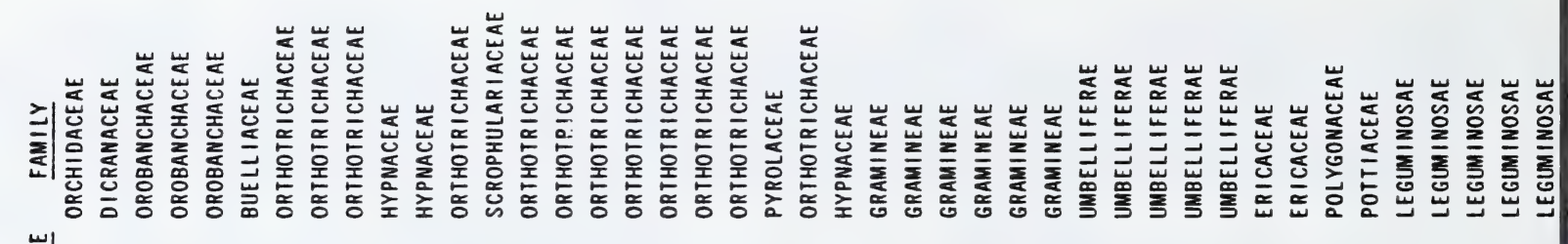

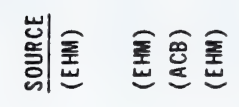

重

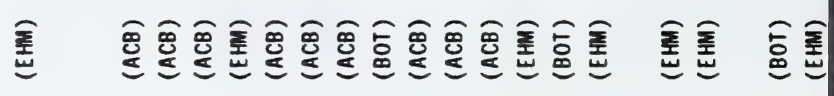
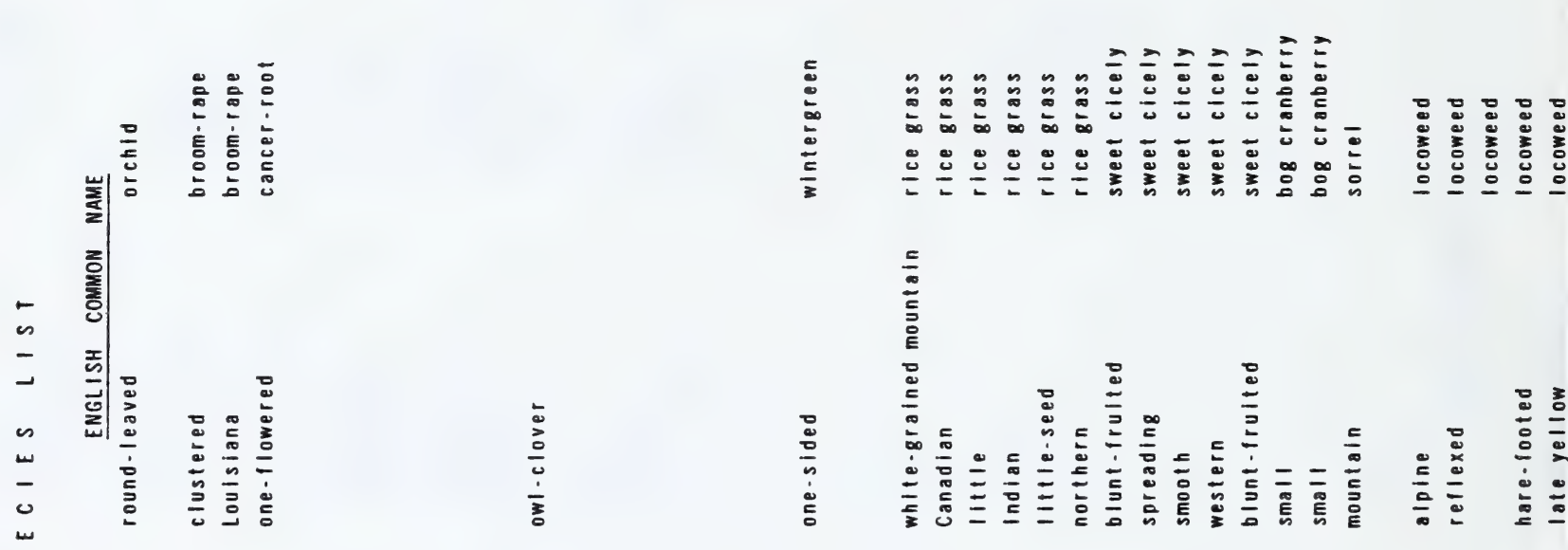

(2)

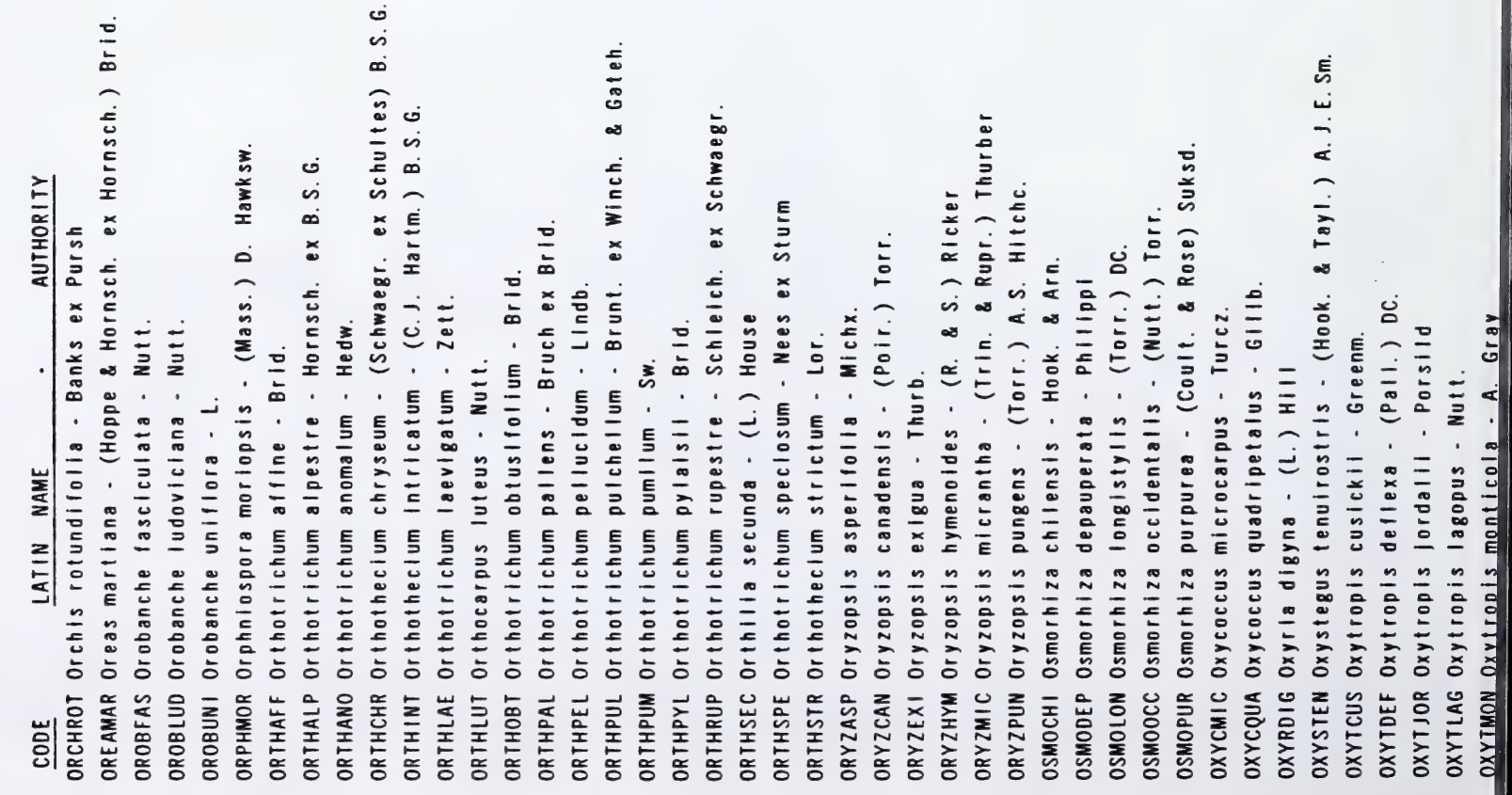


خ|

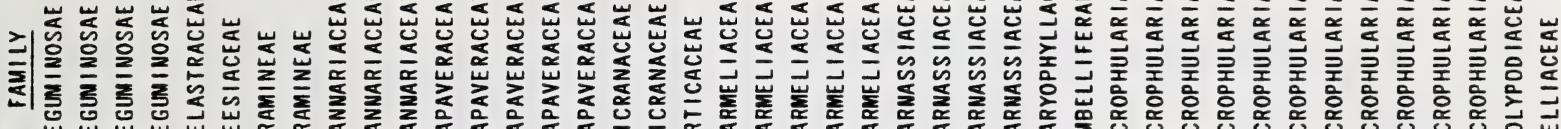

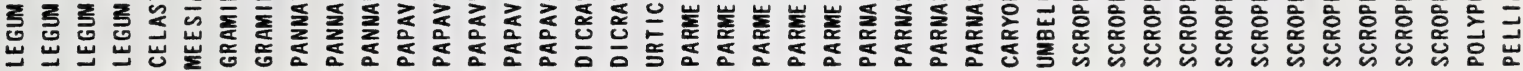

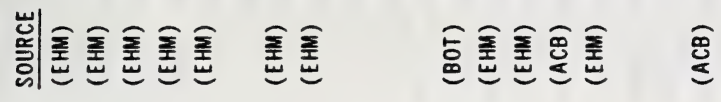

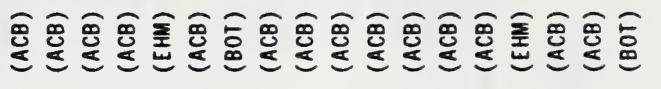

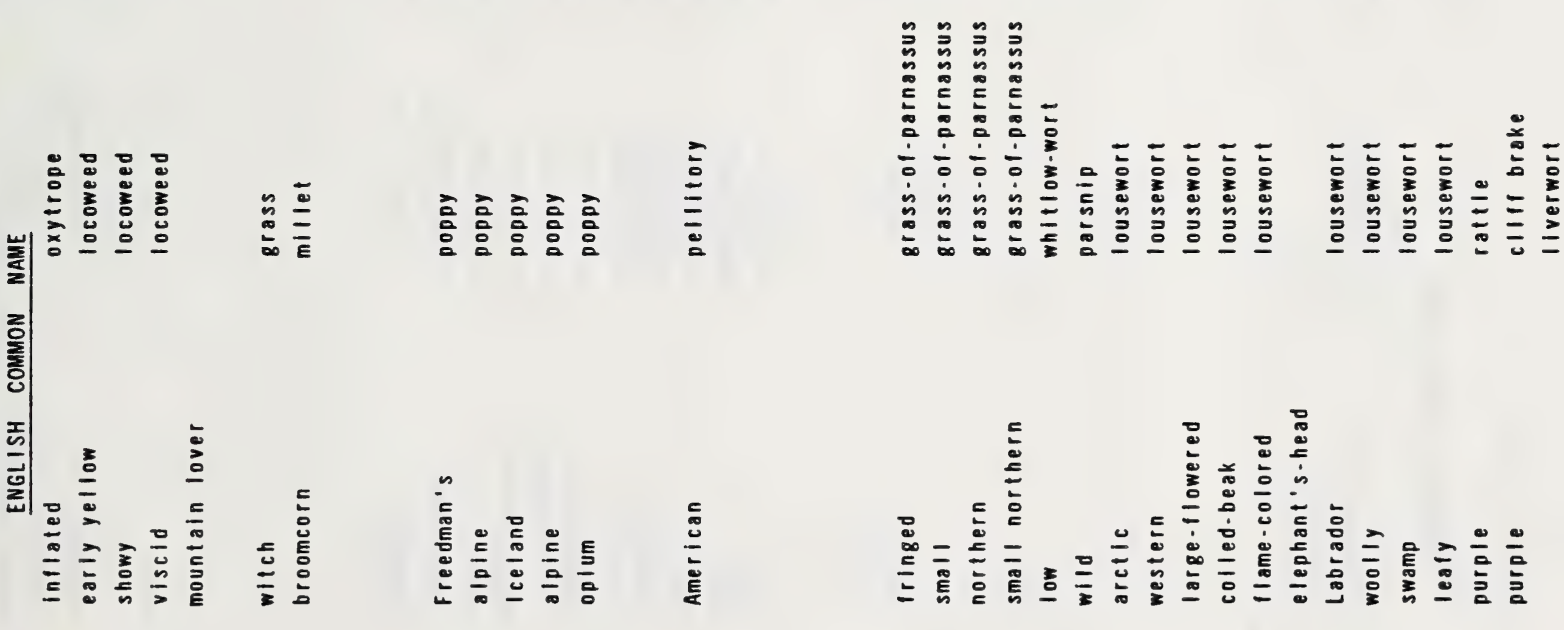

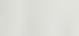 (2)

$\stackrel{5}{\infty}$

产



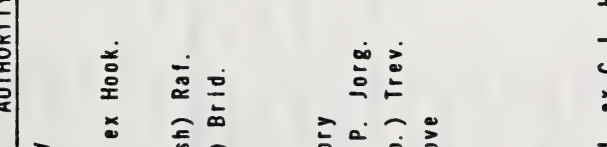

늠

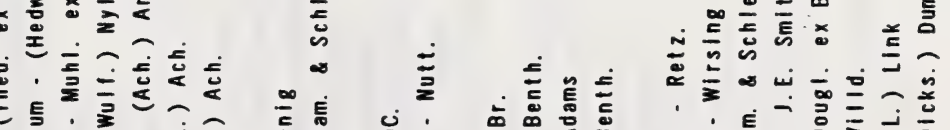

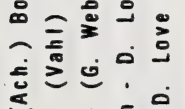

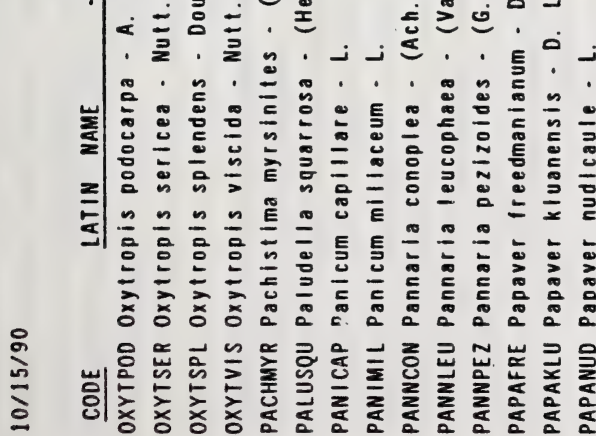

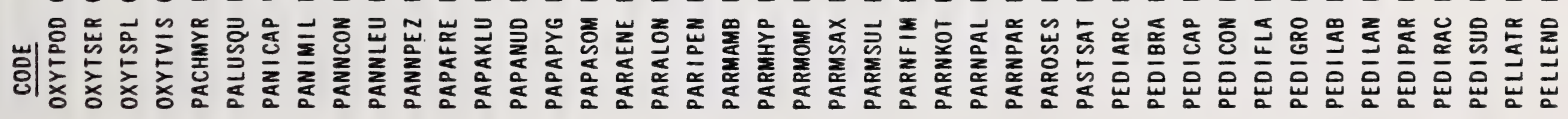




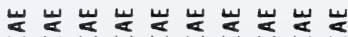

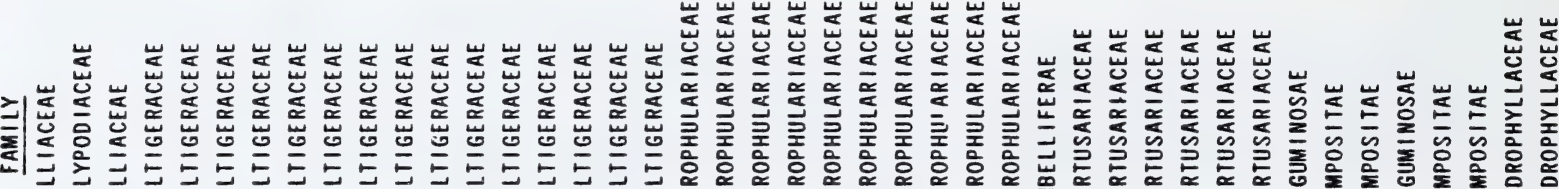

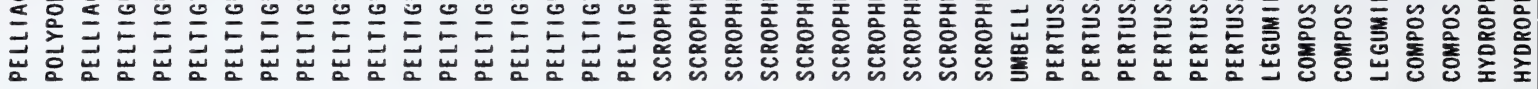
岕兽芯

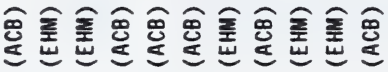

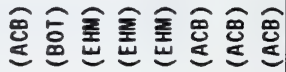
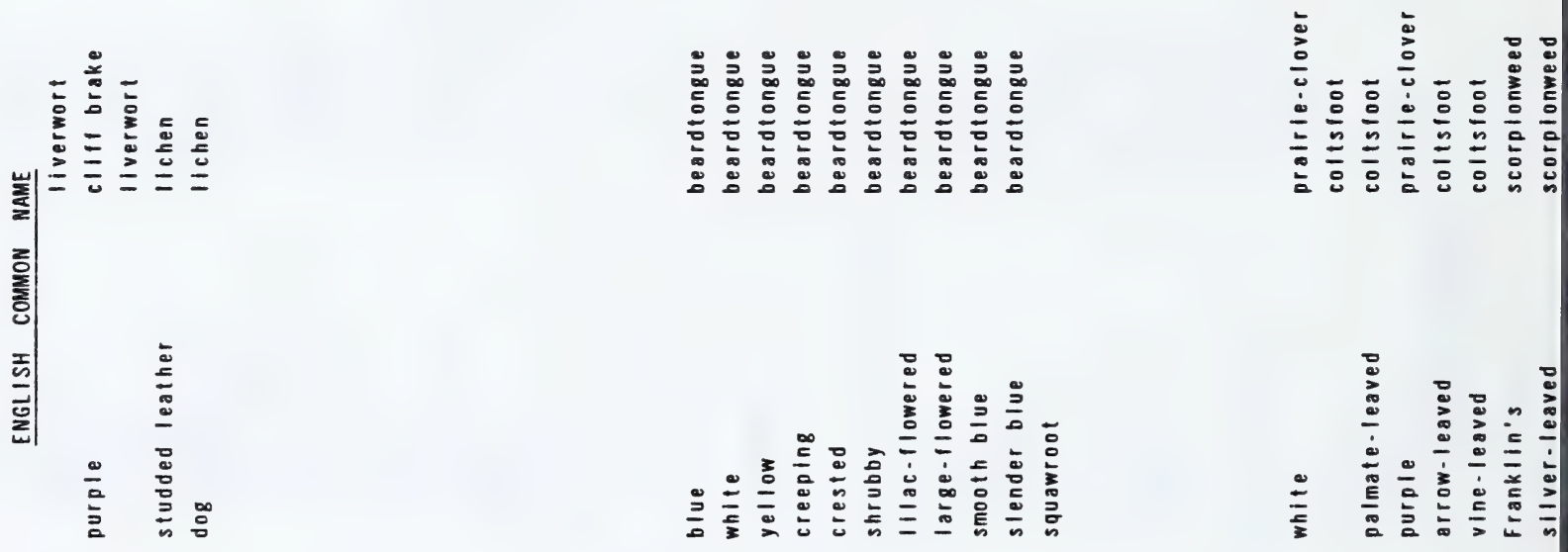

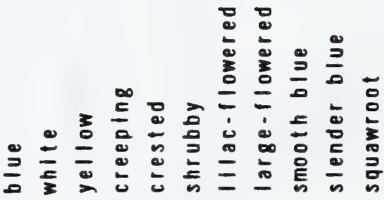
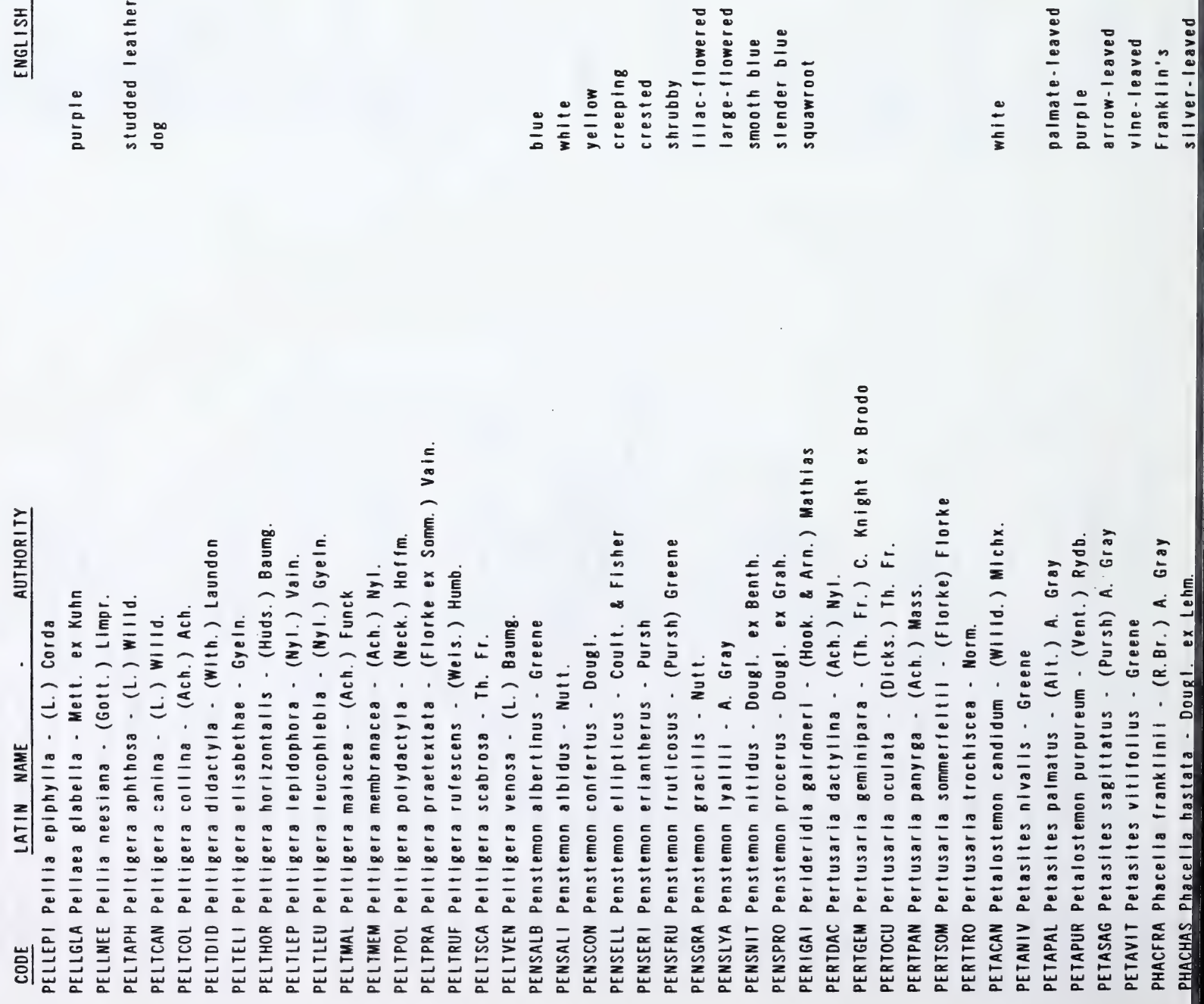
崖崖

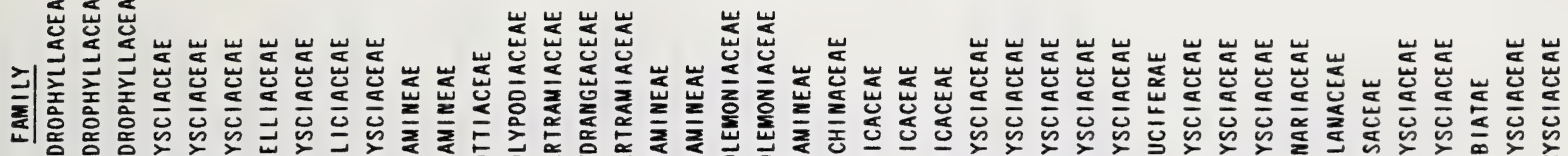

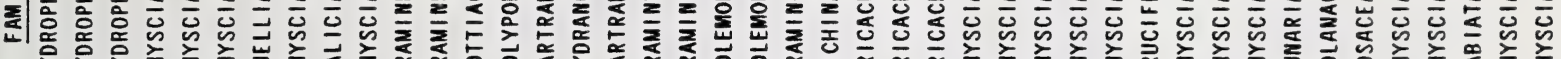

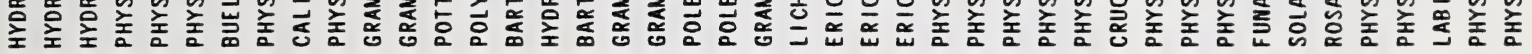

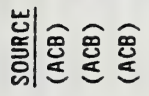

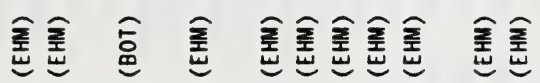

$\widehat{\underline{\underline{x}}}$

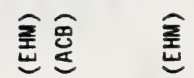

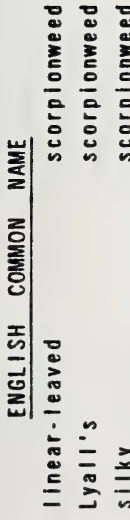

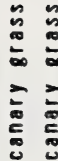

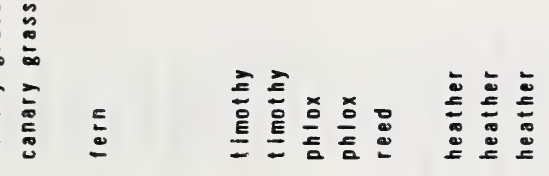

:

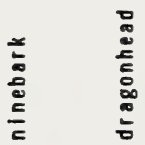

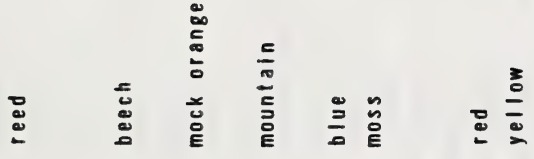

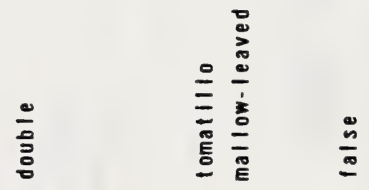

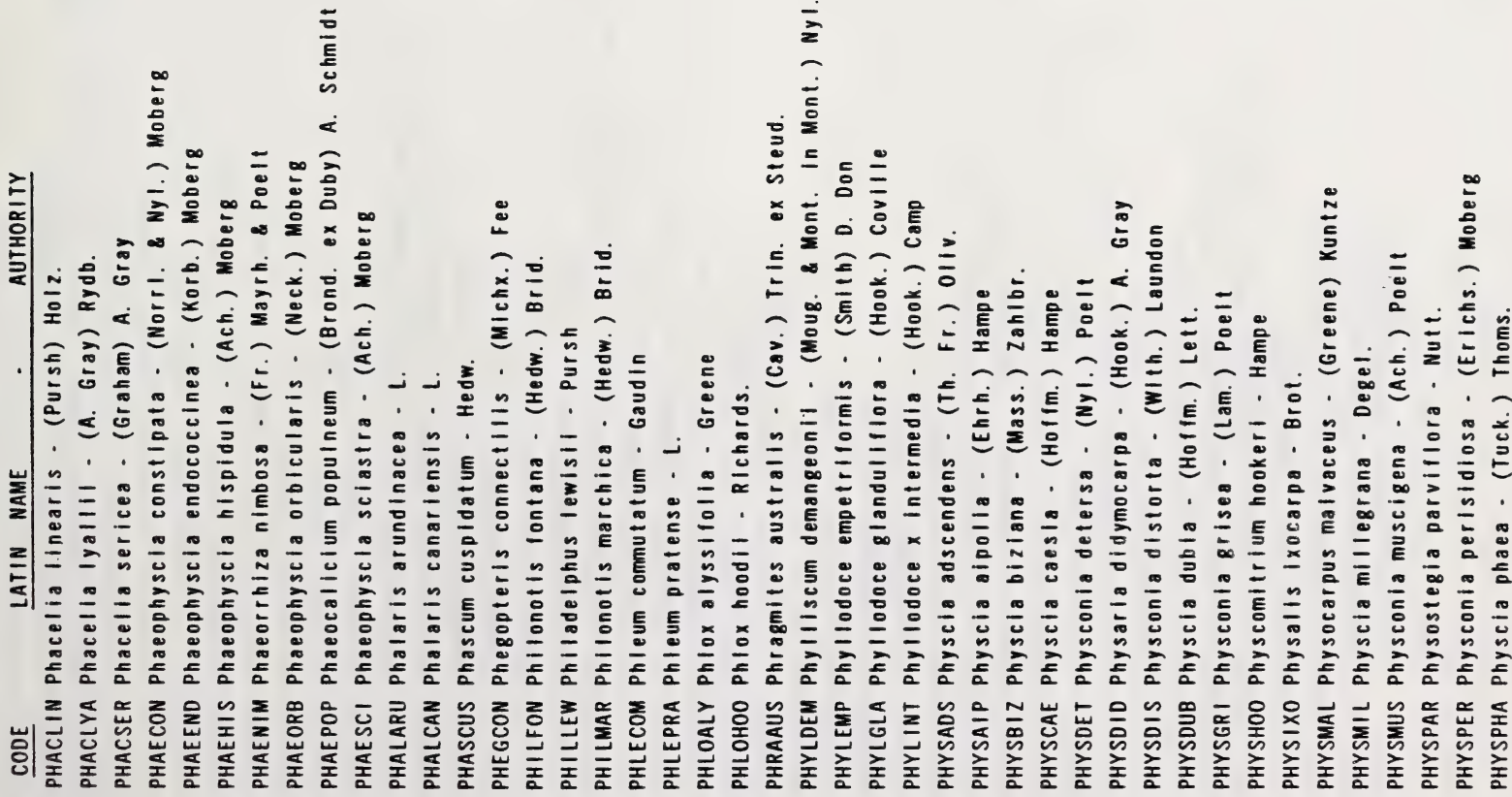




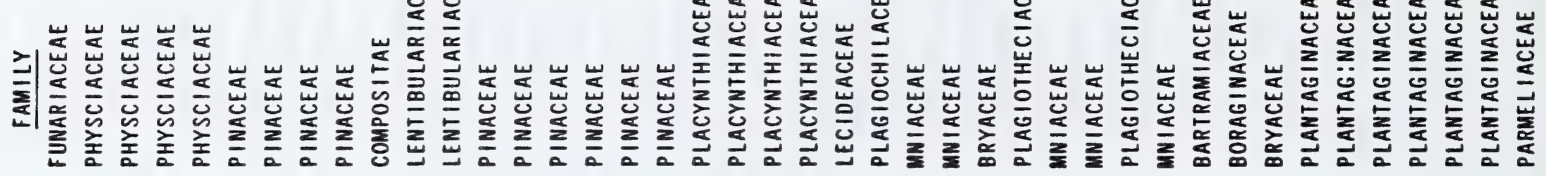

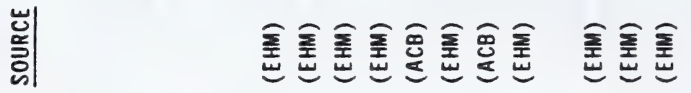

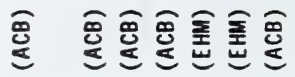
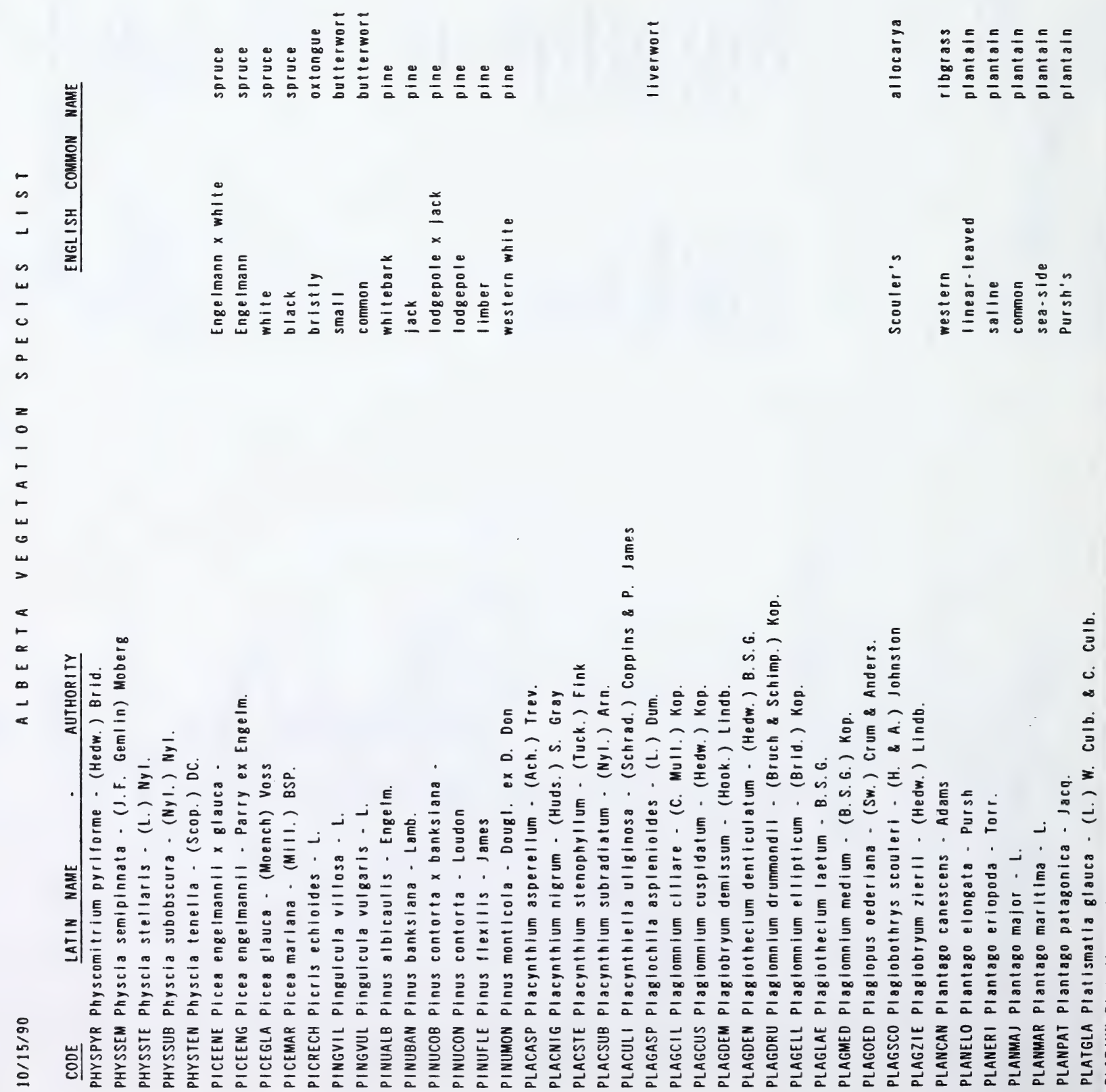
峞

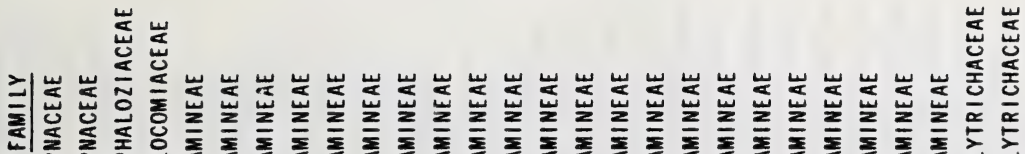

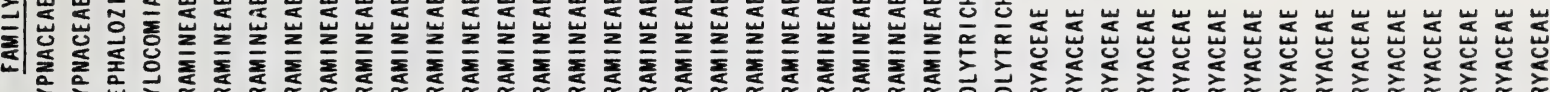

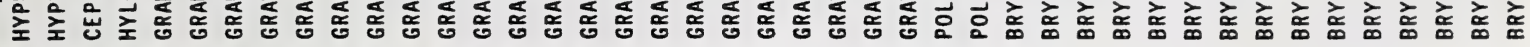

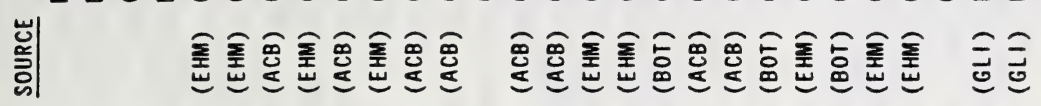

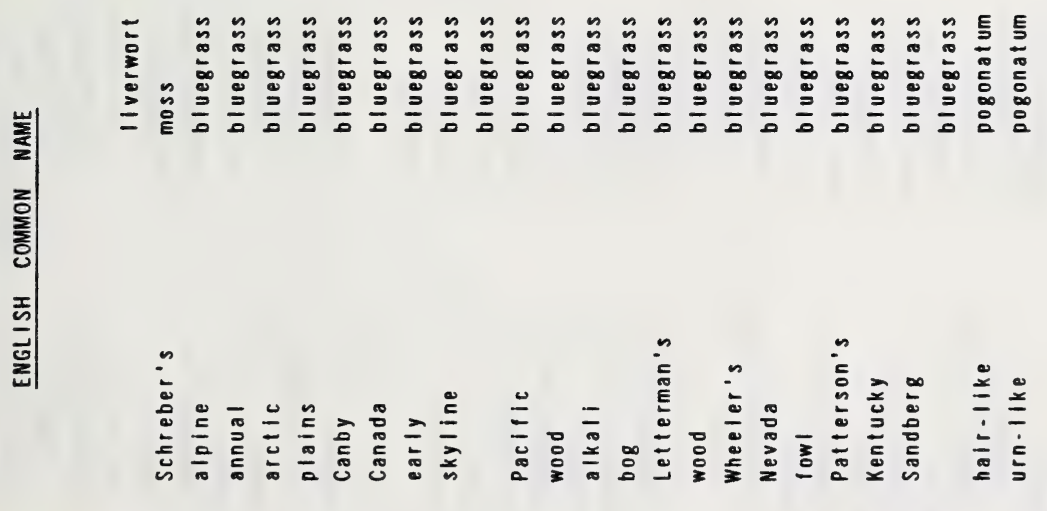

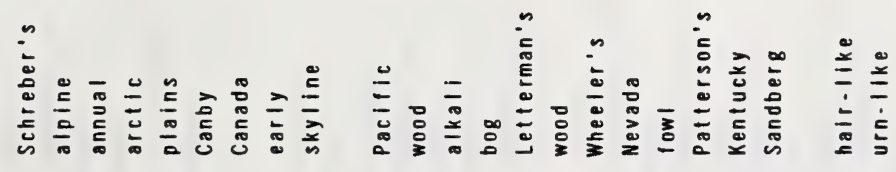

$\frac{\text { E }}{\text { ¿ }}$

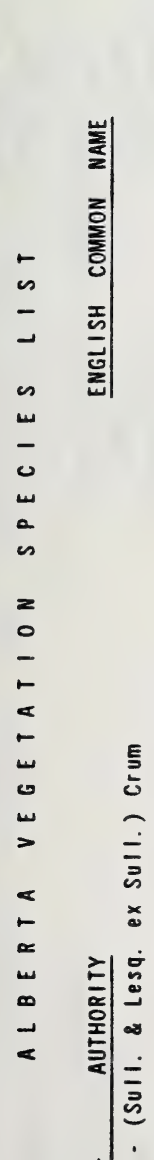

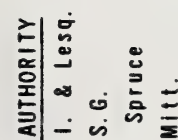

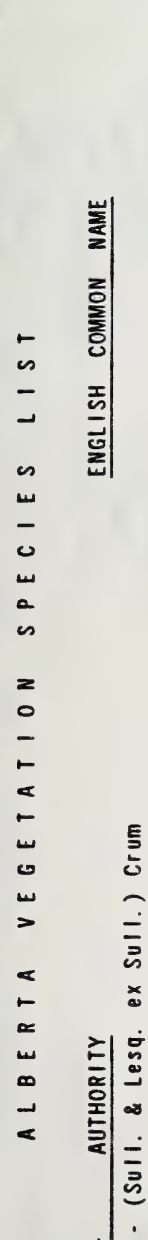

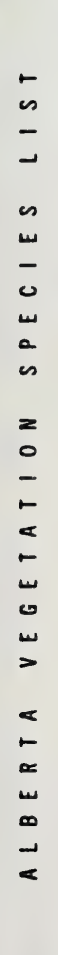

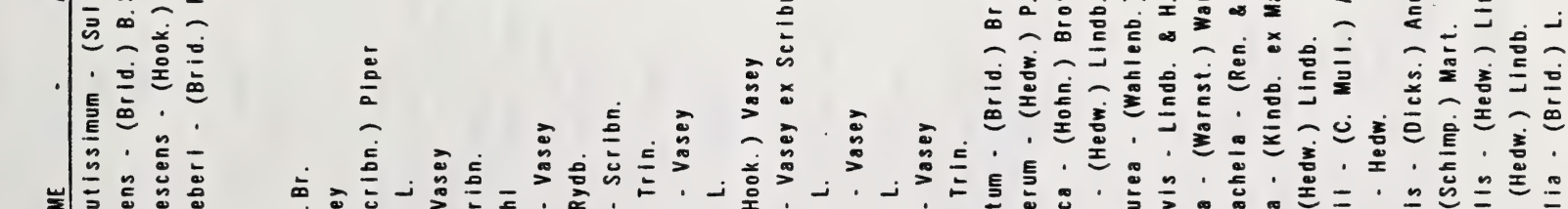

z

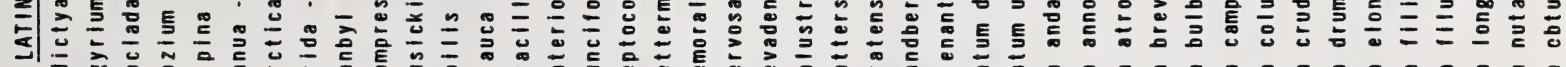

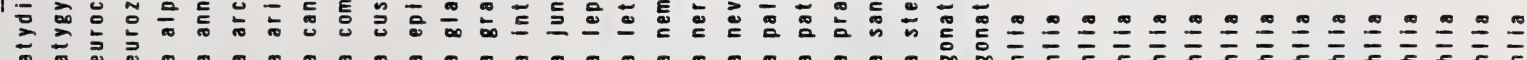

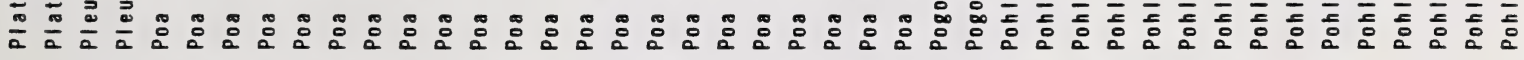




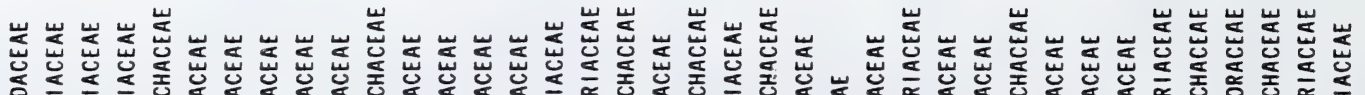

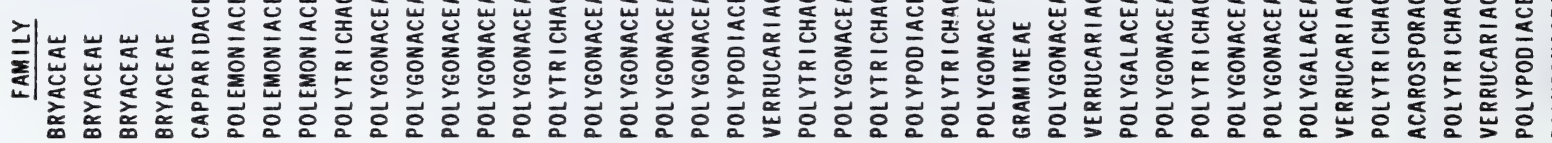
亳|

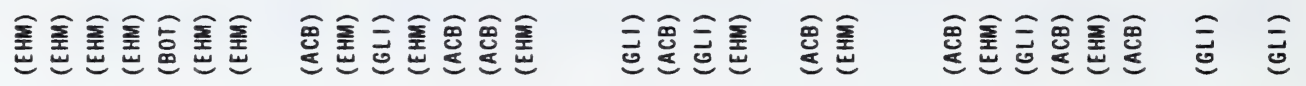

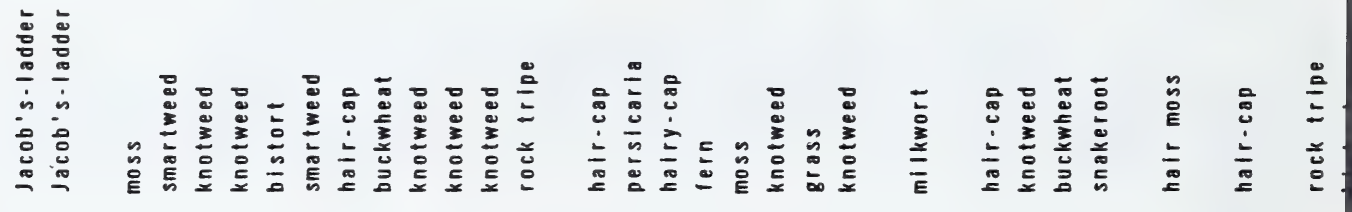

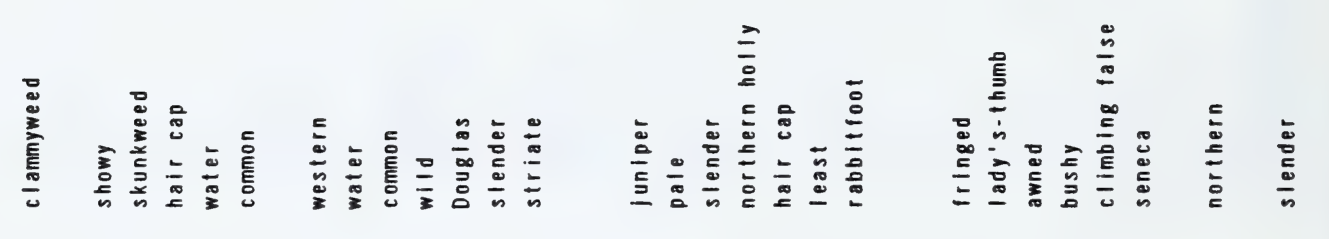

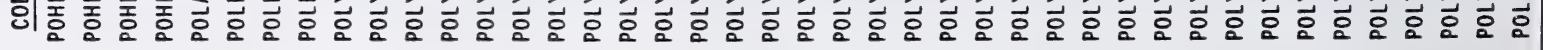

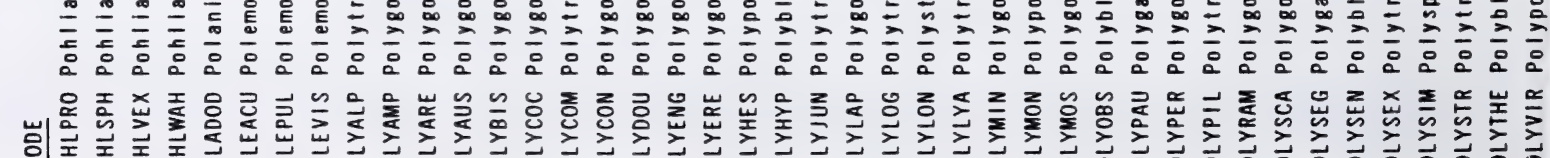




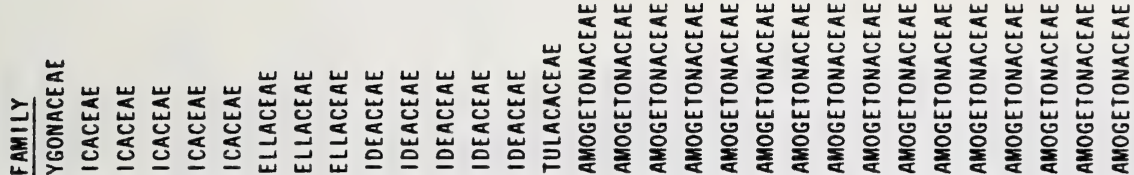

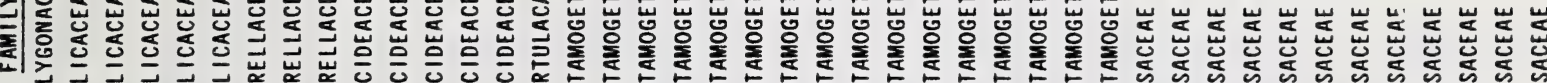

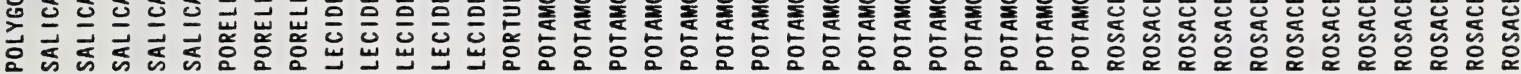

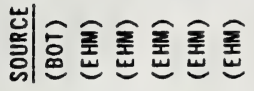

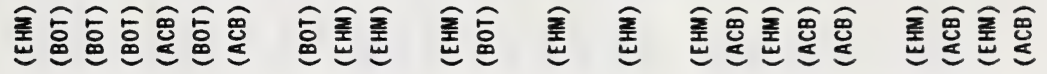
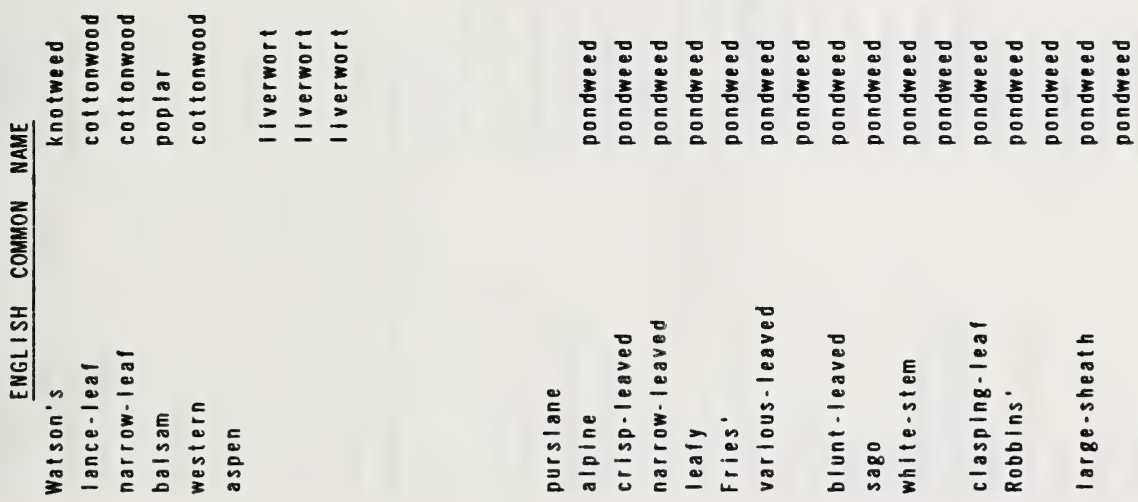

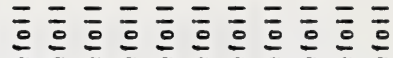

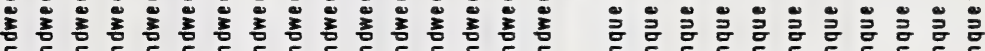
응:

$$
\begin{aligned}
& \vdots \\
& \vdots \\
& \vdots \\
& \vdots \\
& \vdots \\
& \vdots \\
& \vdots
\end{aligned}
$$




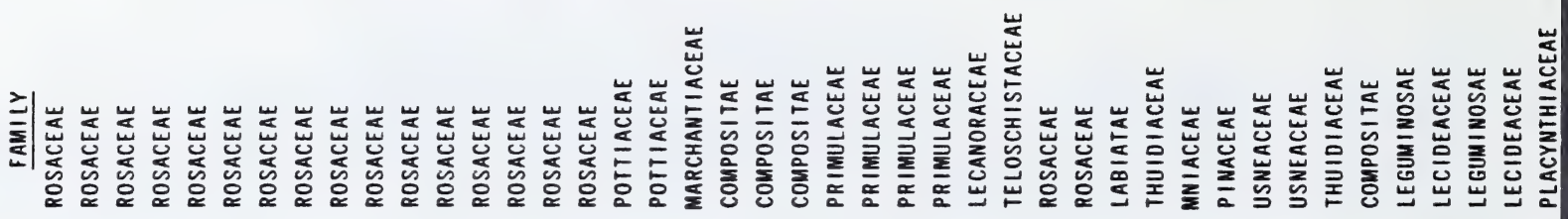

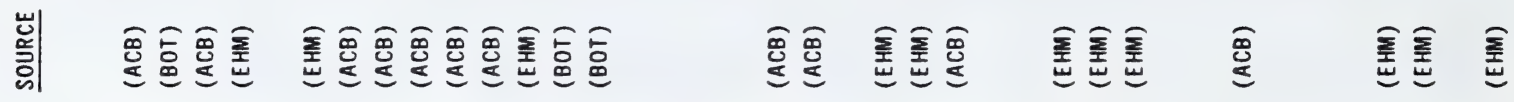

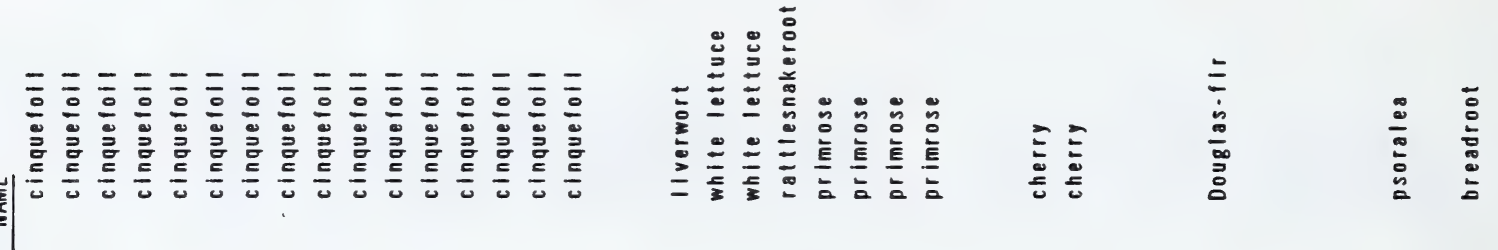

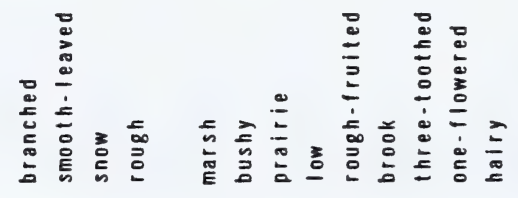

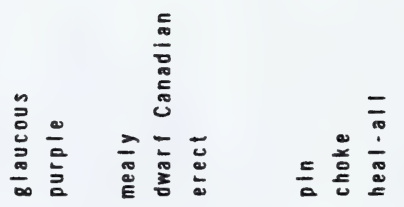

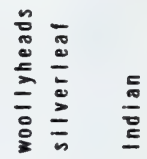




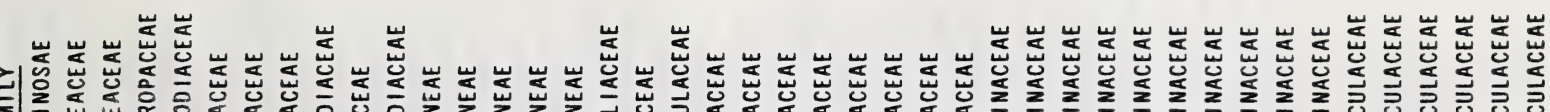

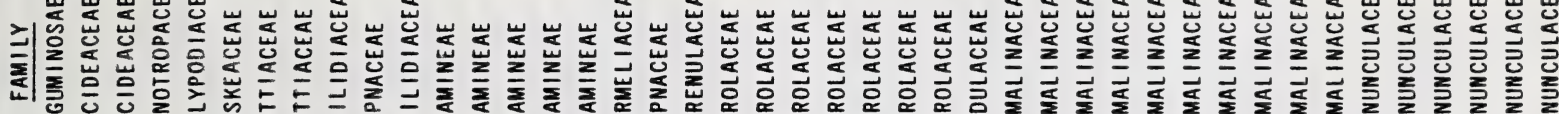

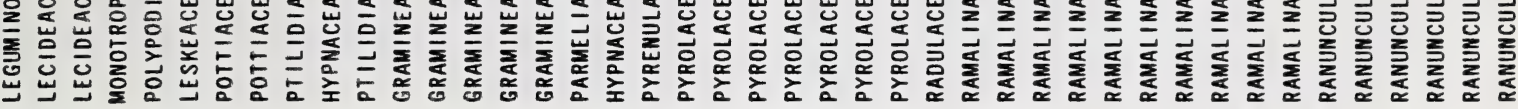

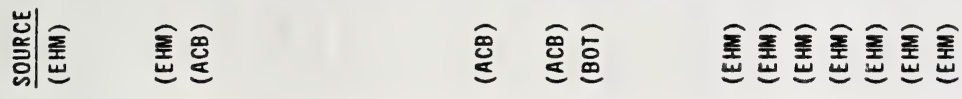

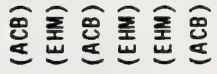

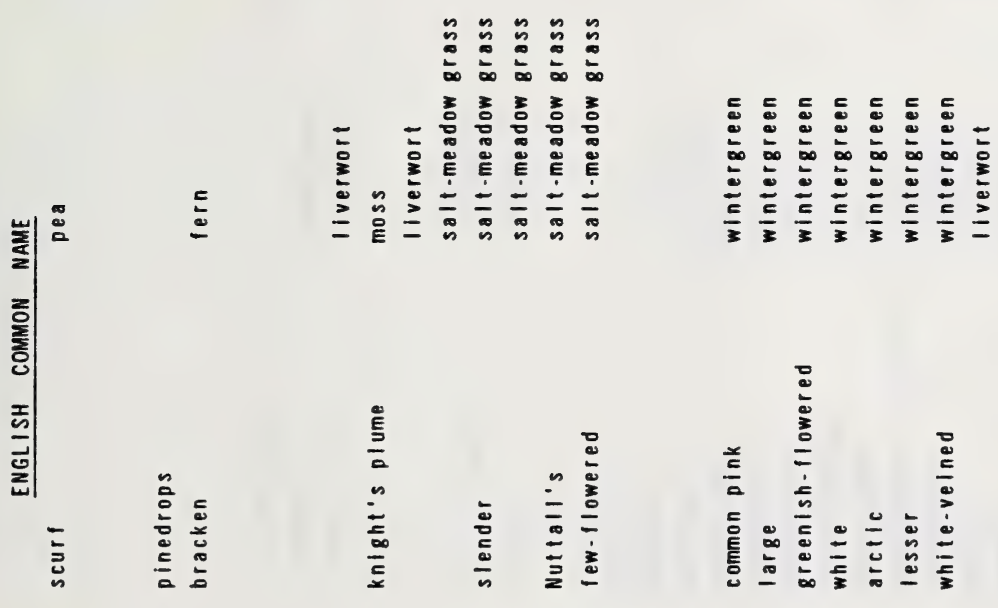

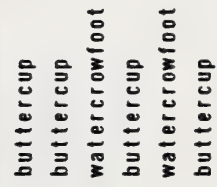

is

$$
\begin{aligned}
& \vdots \\
& \vdots \\
& \vdots \\
& \vdots \\
& \vdots \\
& \vdots \\
& \vdots
\end{aligned}
$$

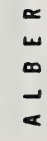

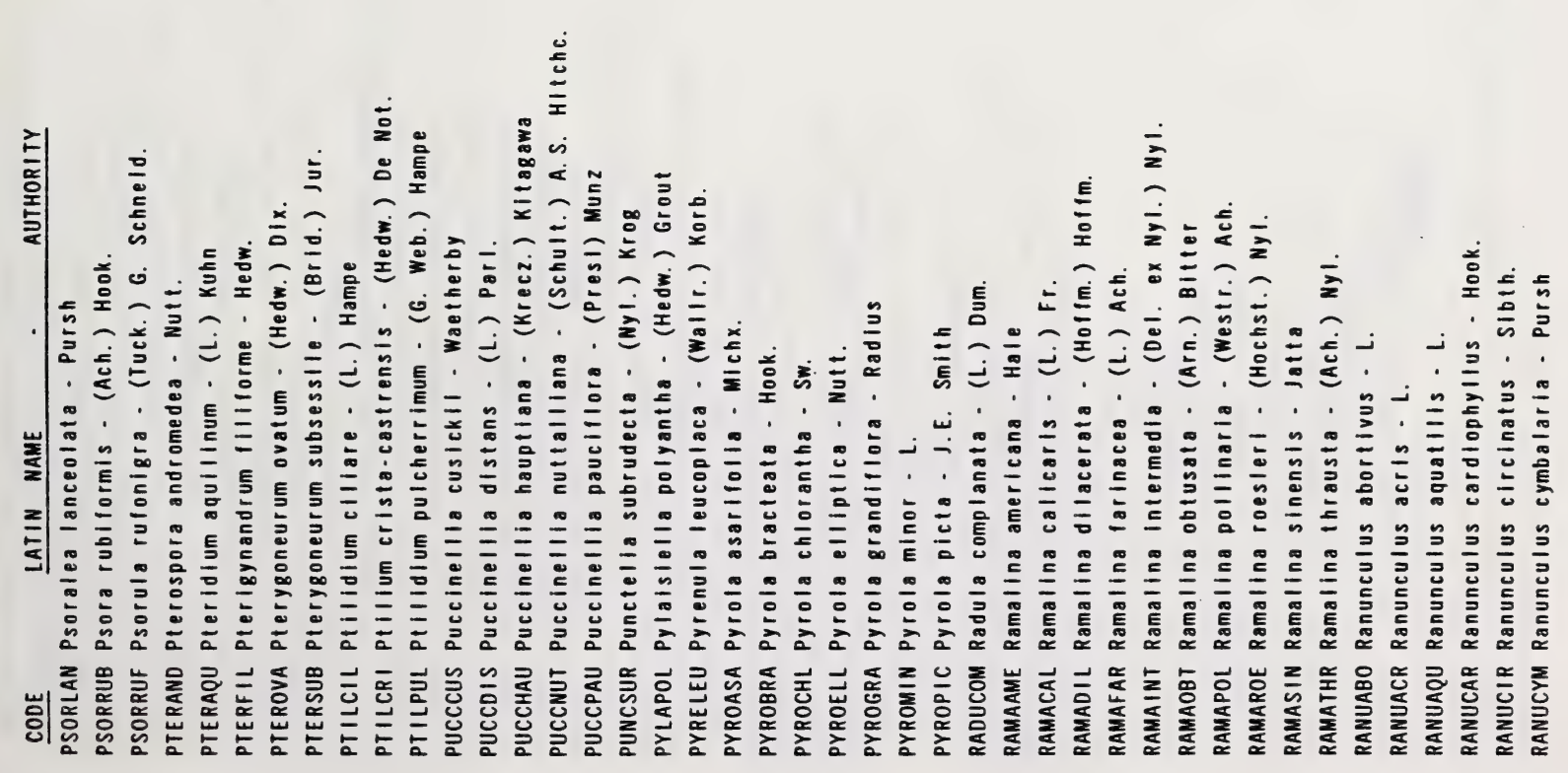




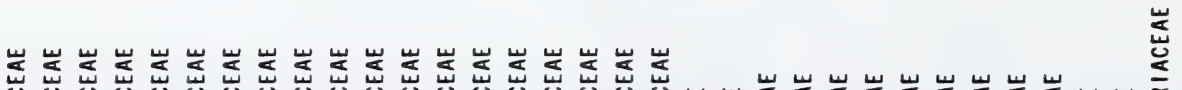

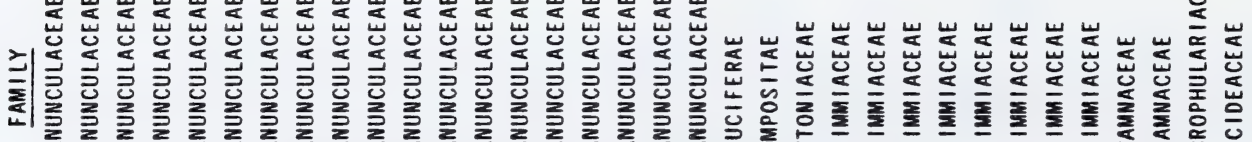

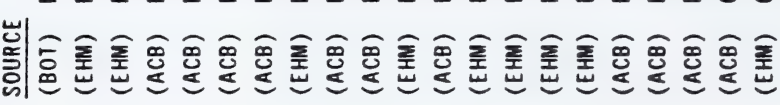
要畐重

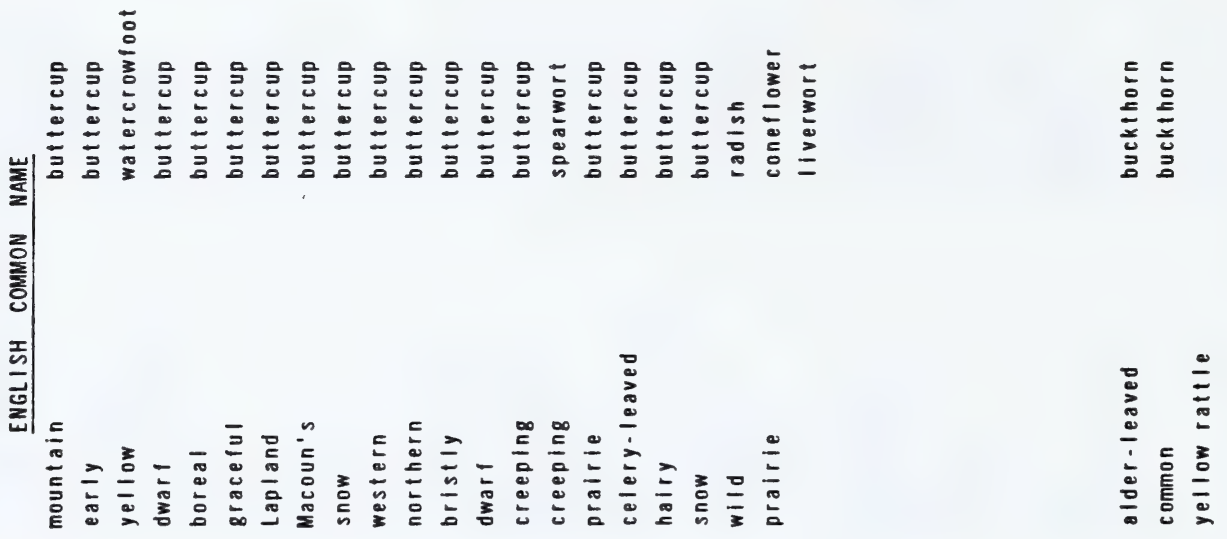

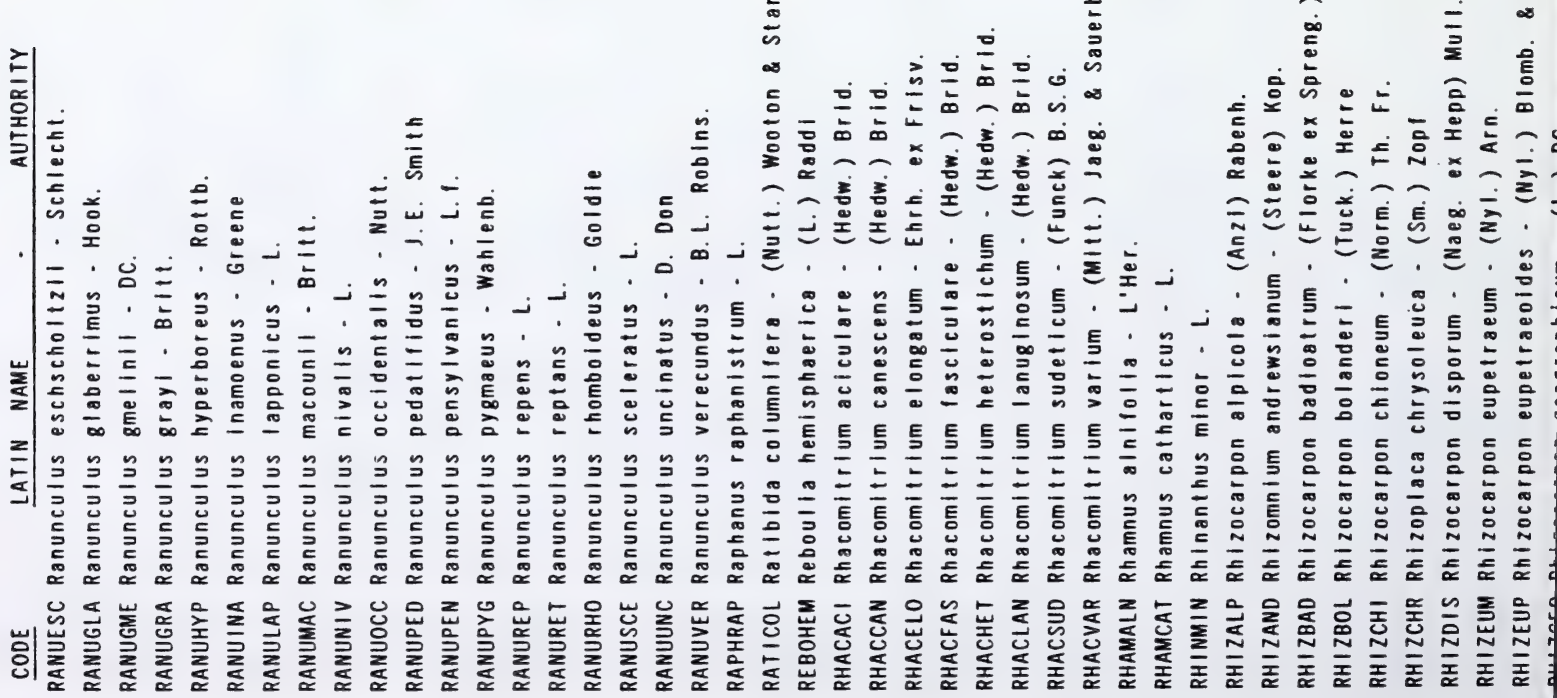




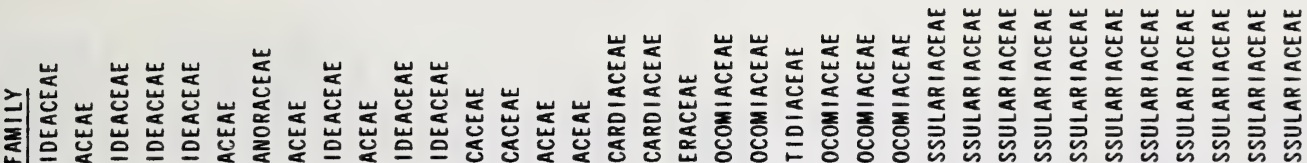

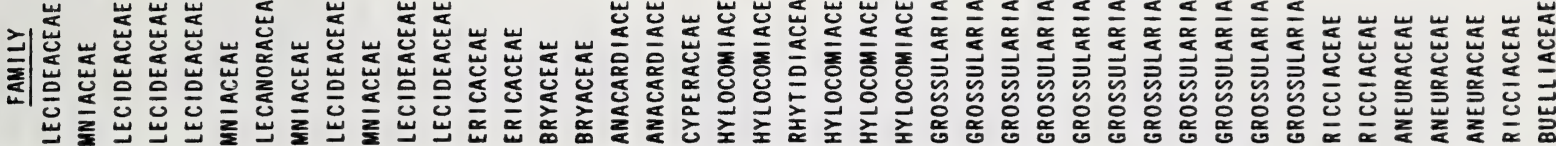

絮

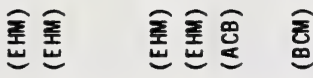

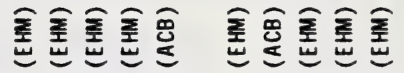
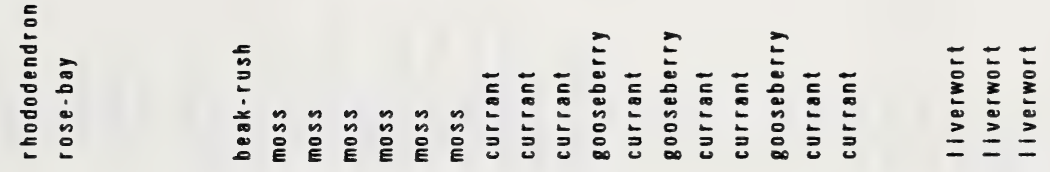

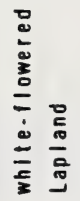

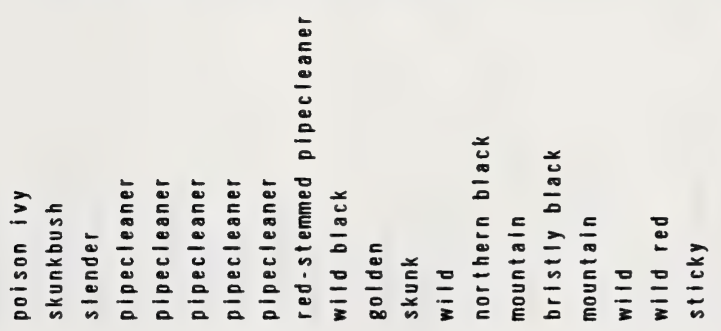

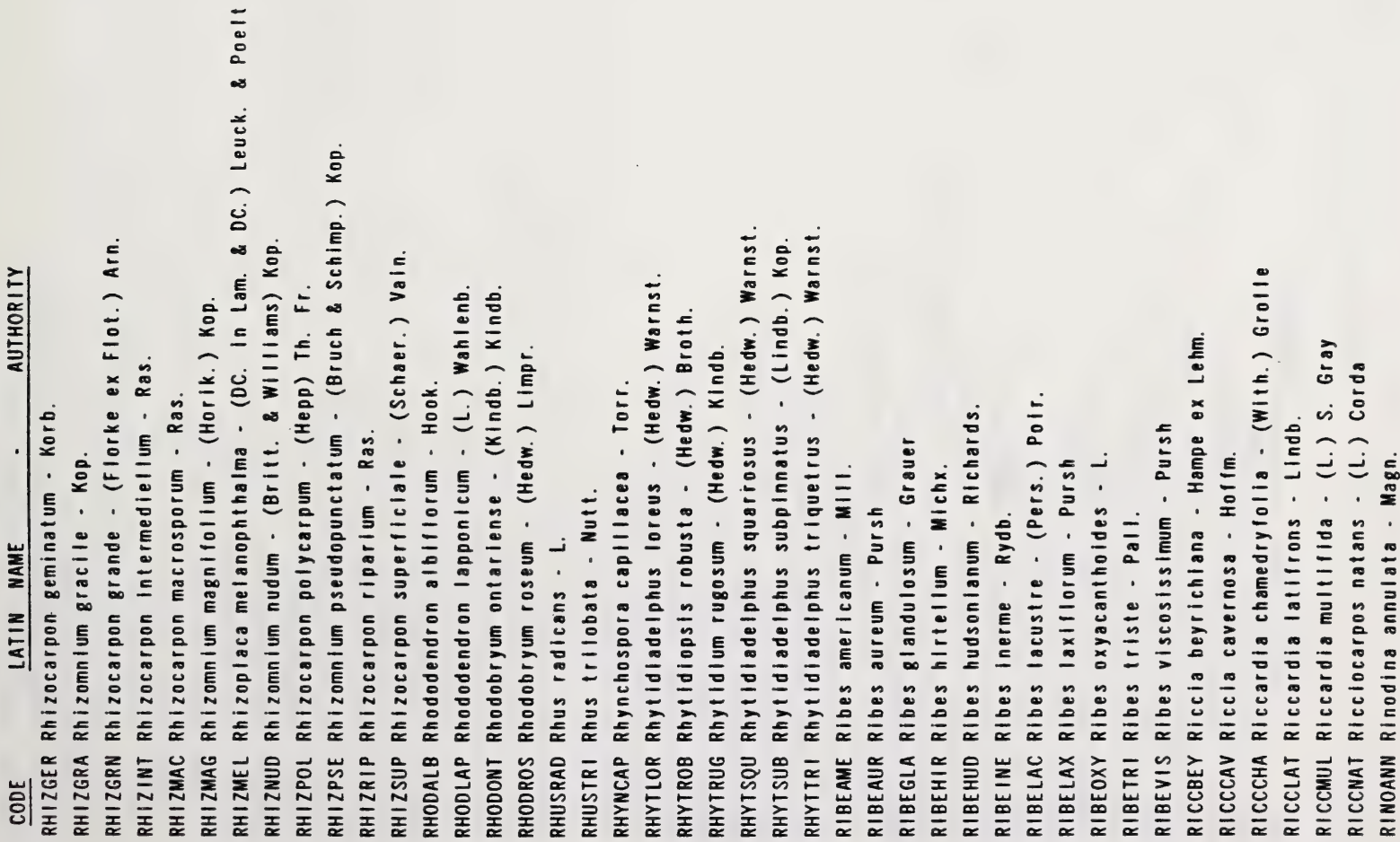




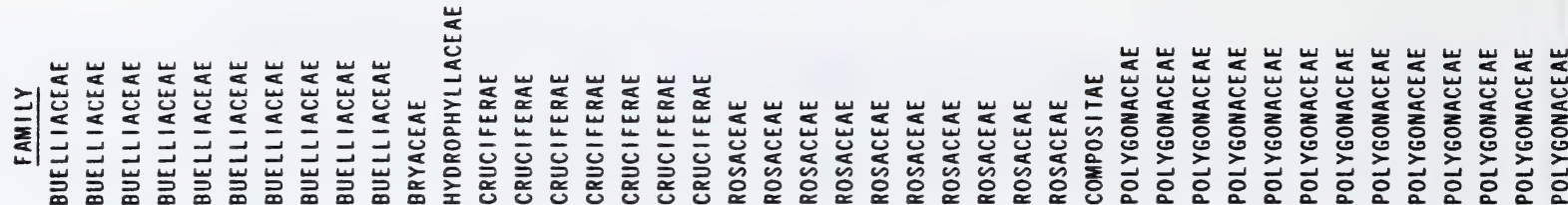
訔

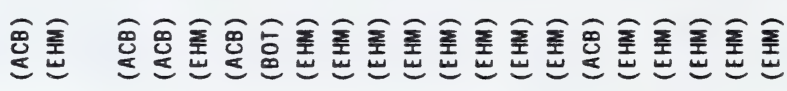

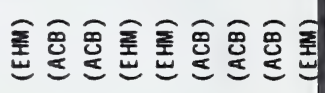

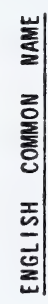

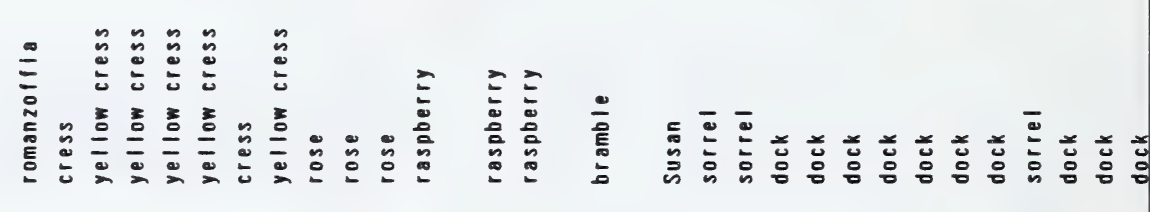

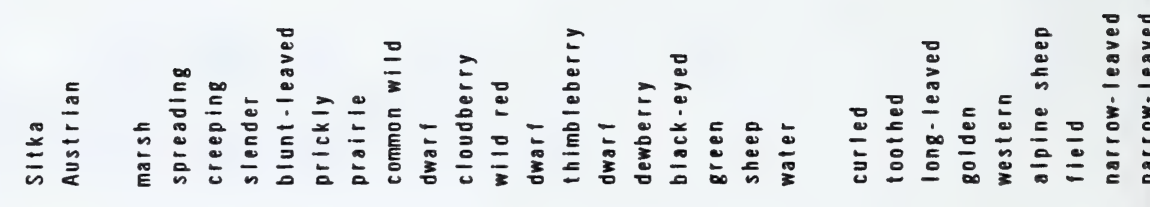

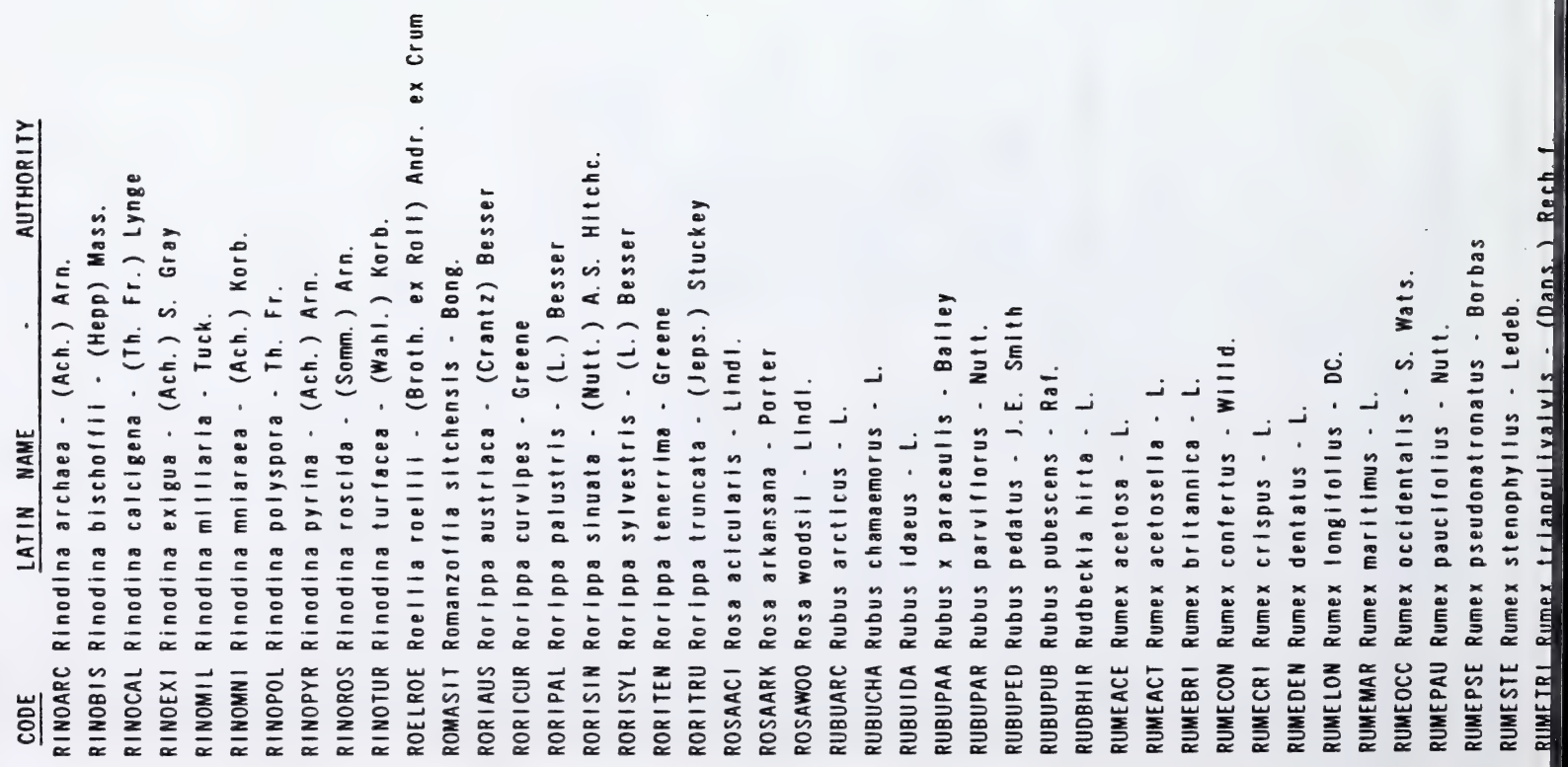




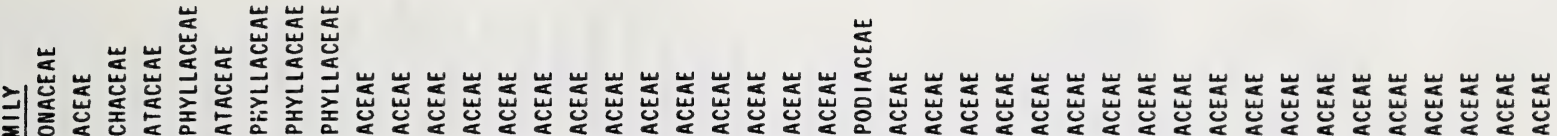

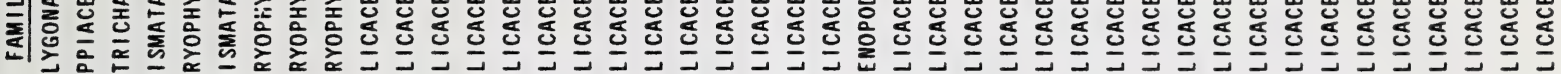

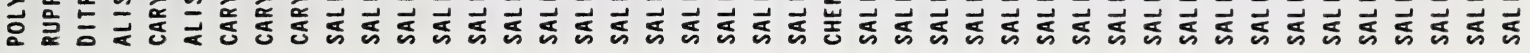

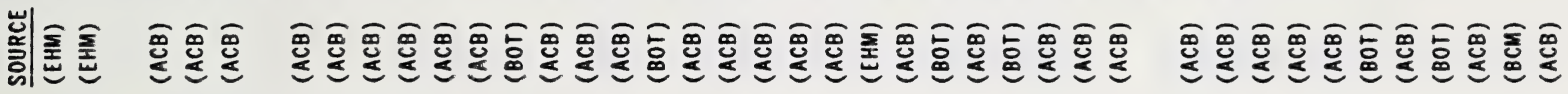

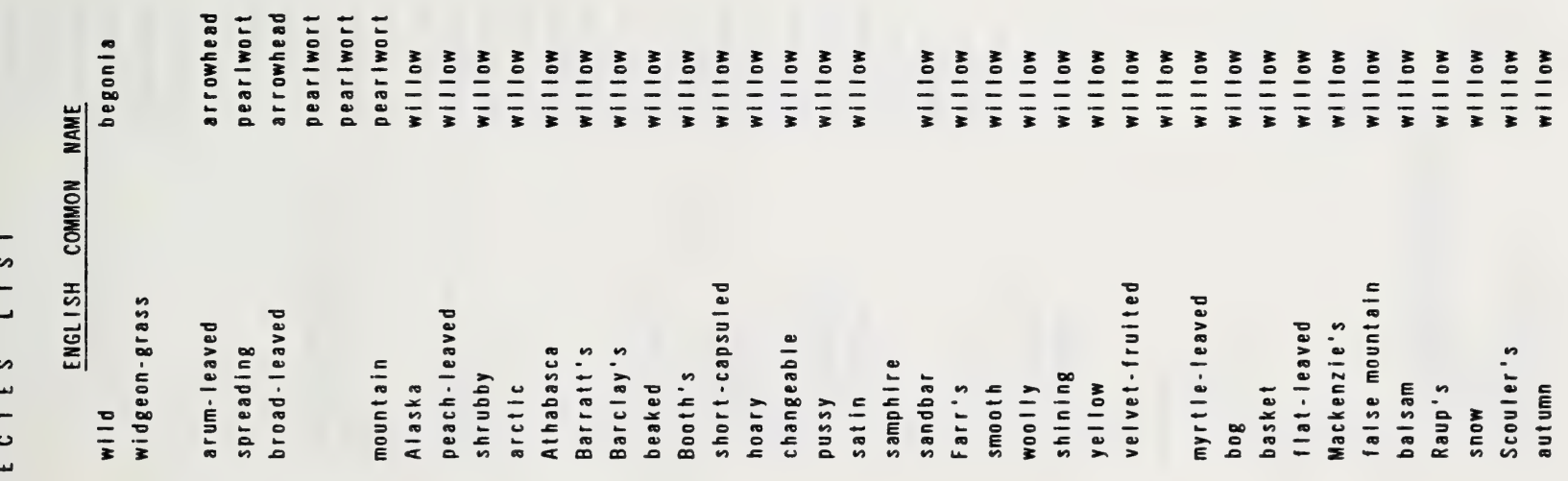

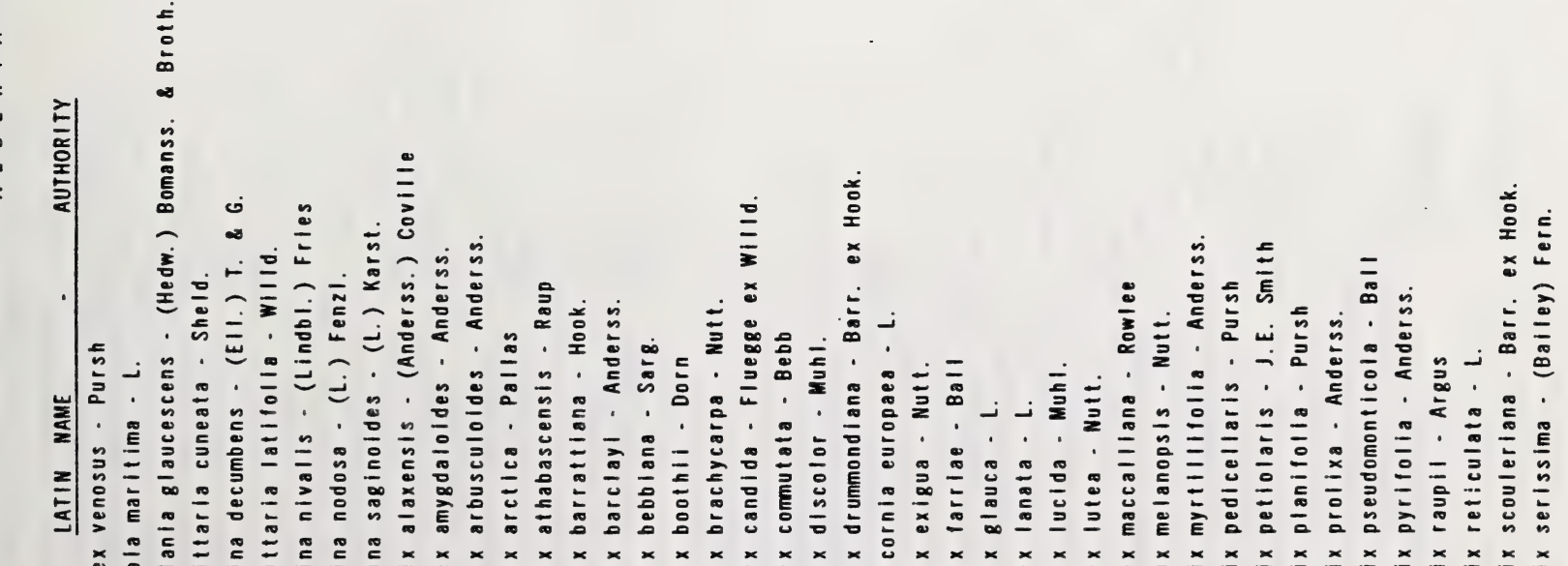

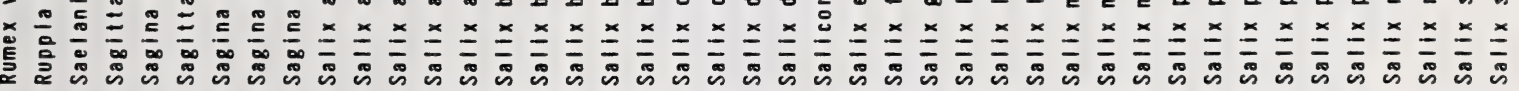
告 


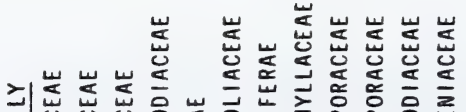

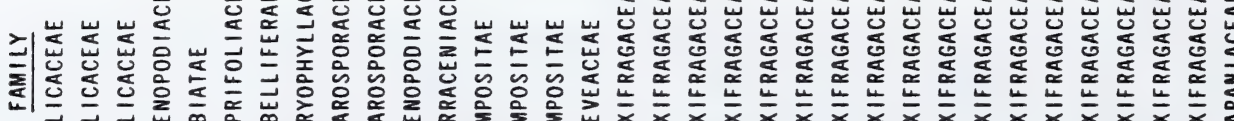

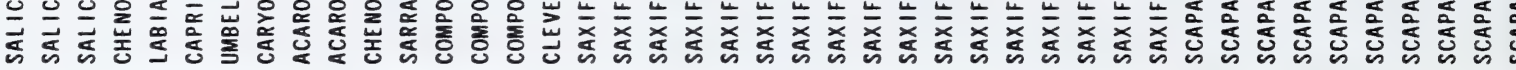

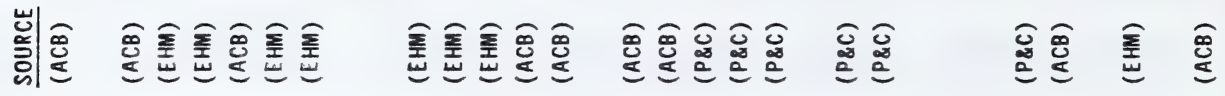
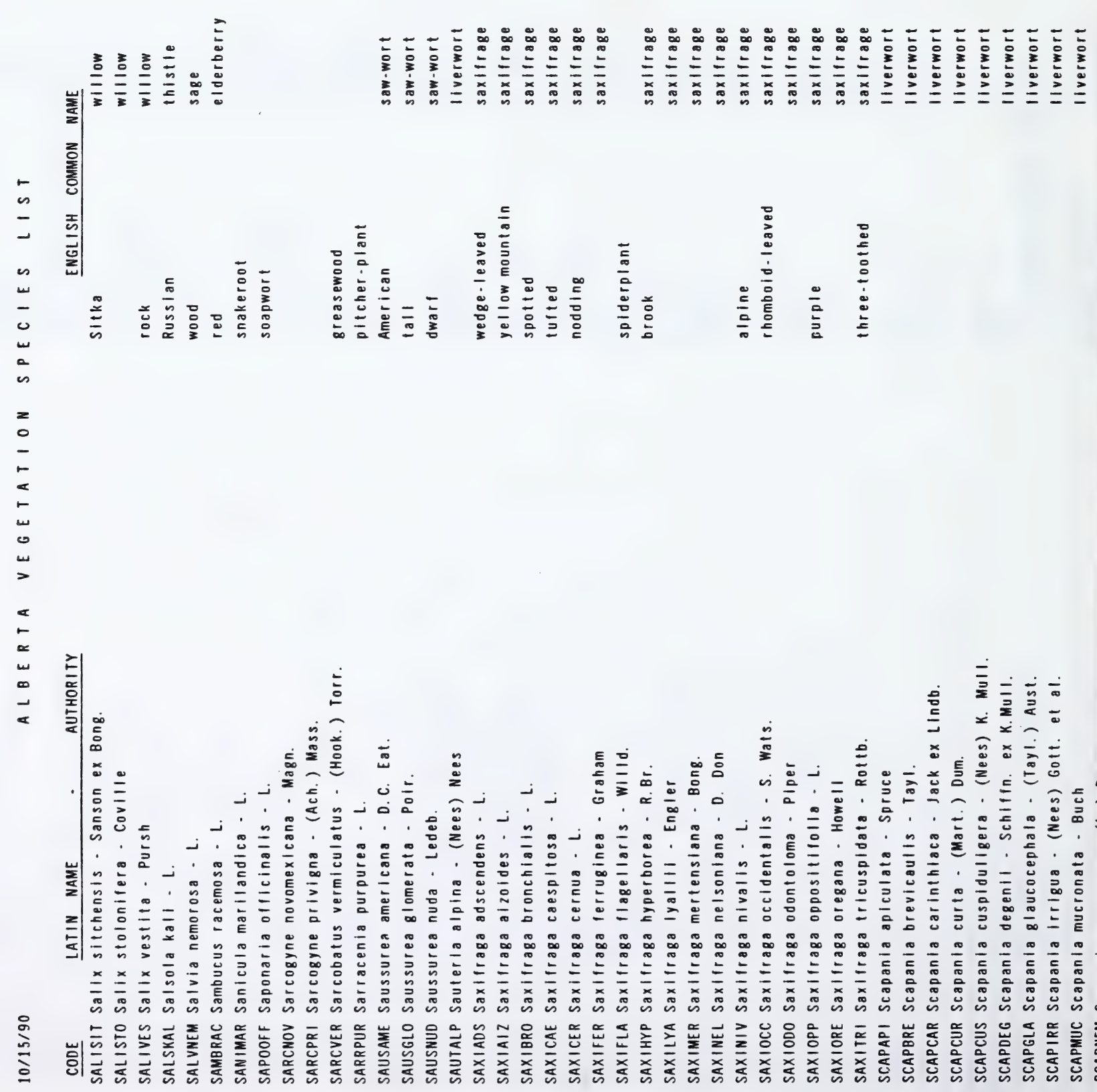


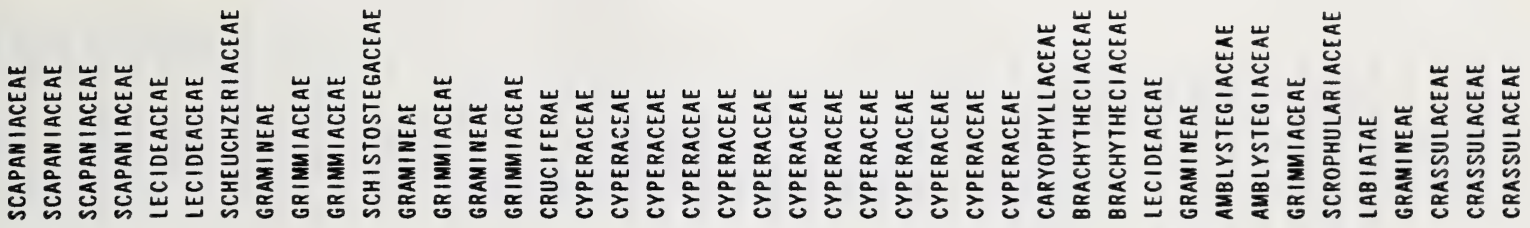
엄

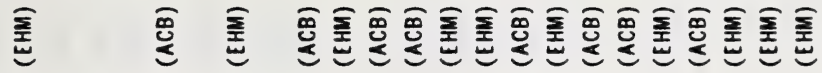

总

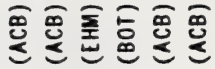

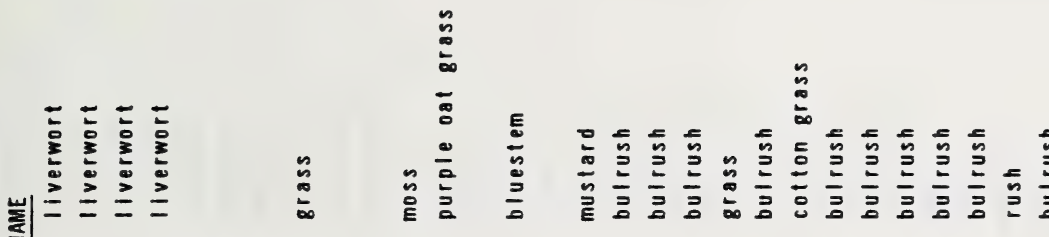

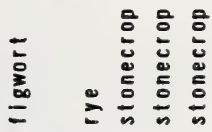

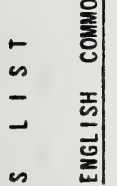

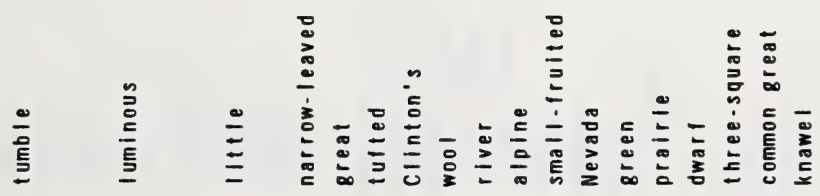

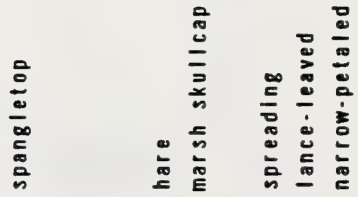

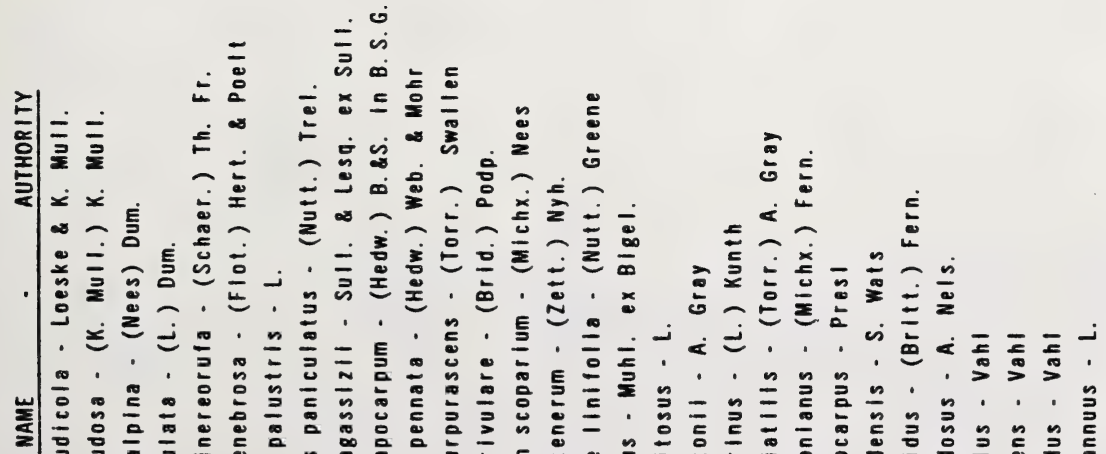

z

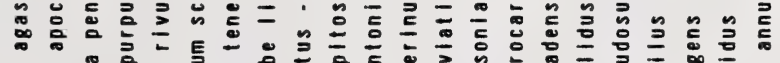

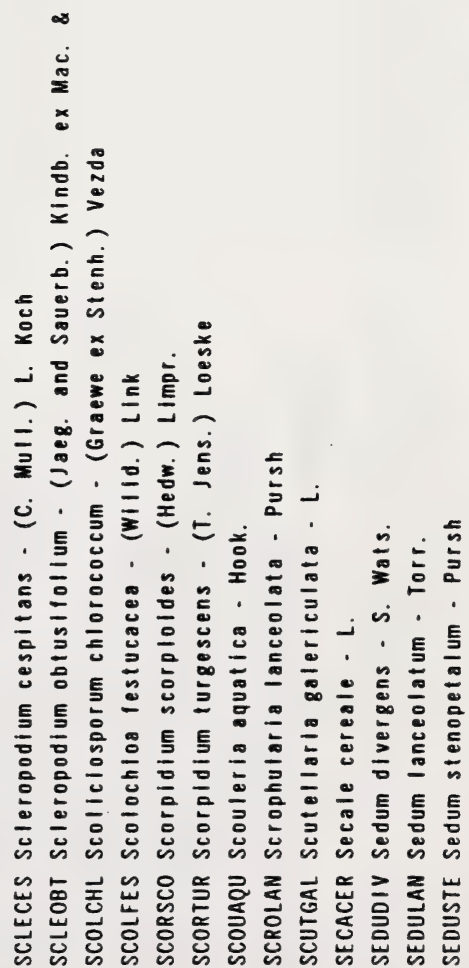


崖㞤崖

岕岕岕岕岕岕岕

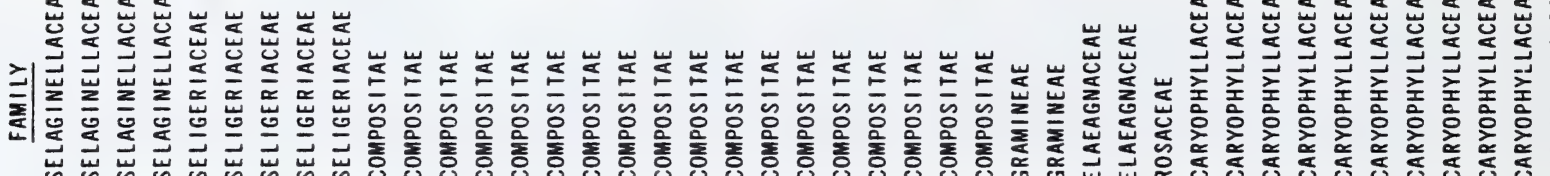
悗

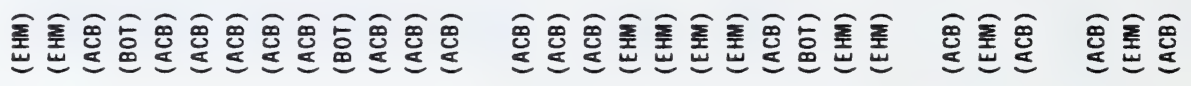
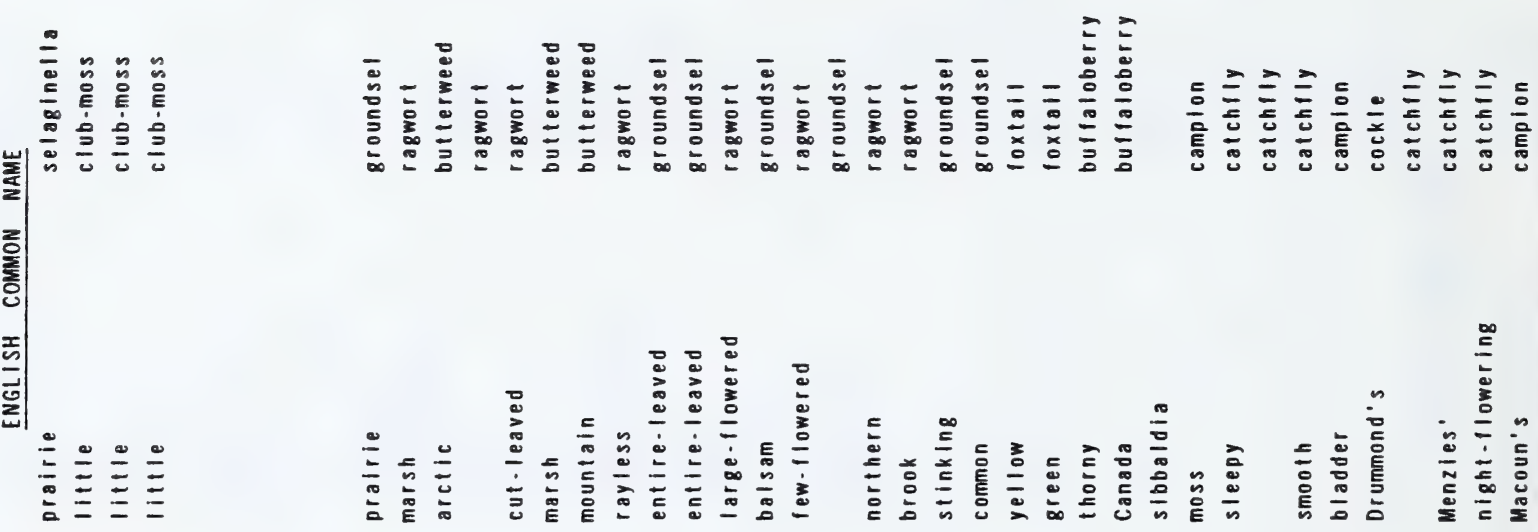

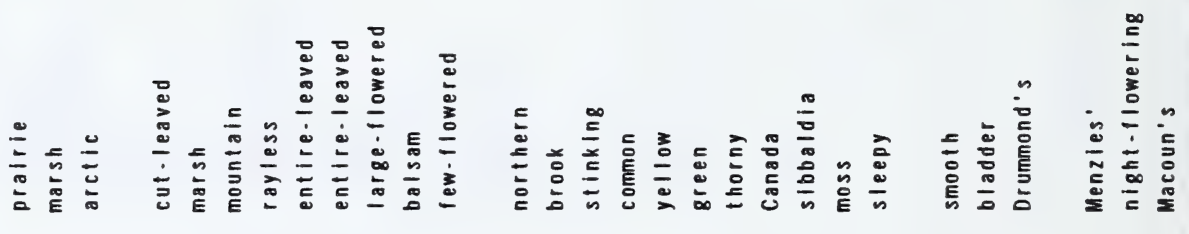

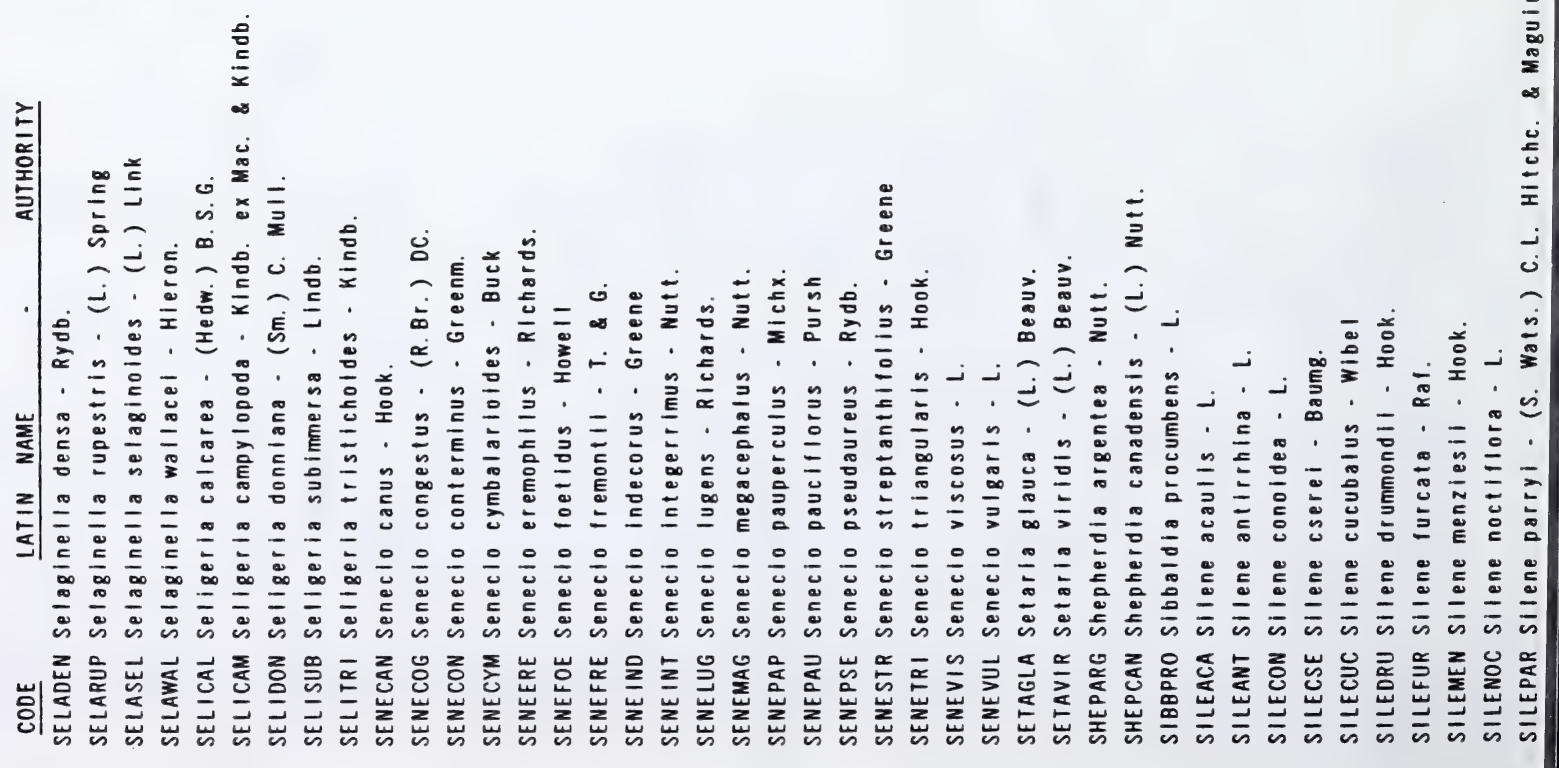




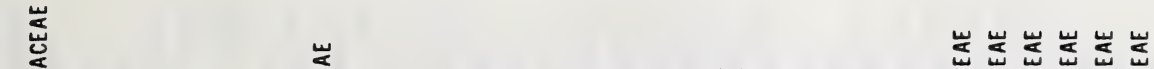

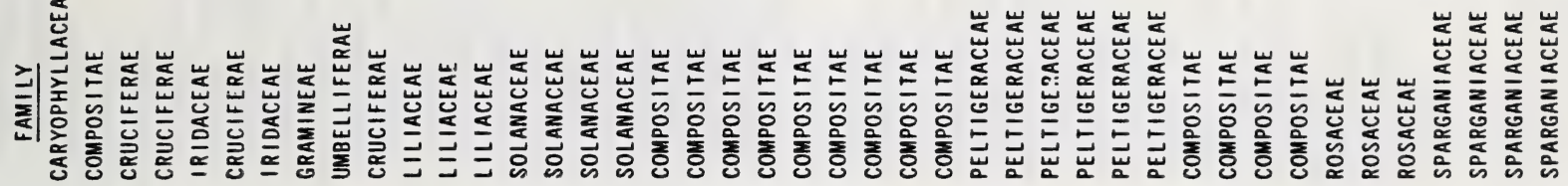

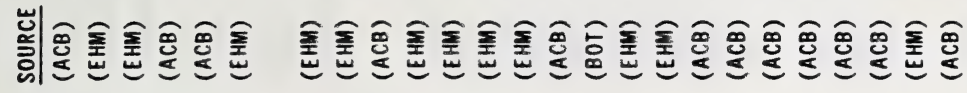

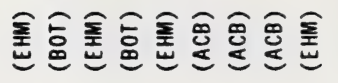

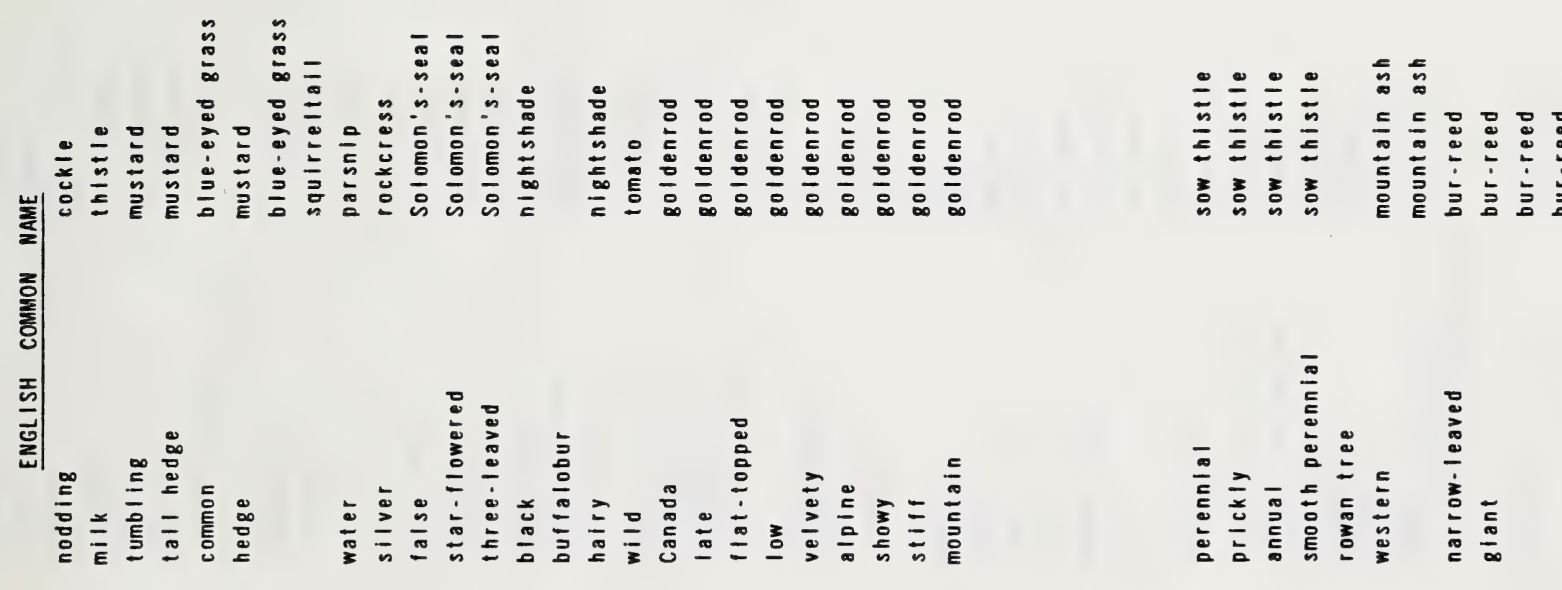

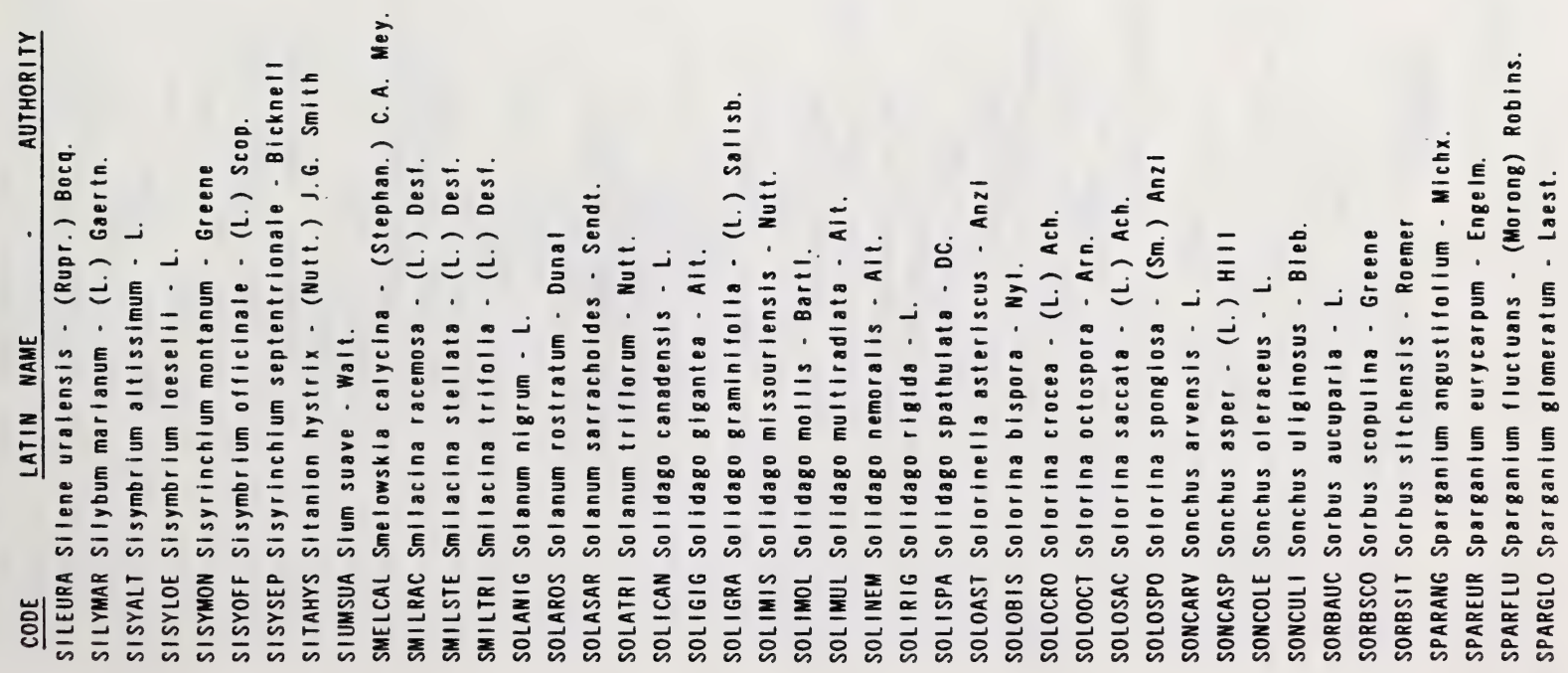




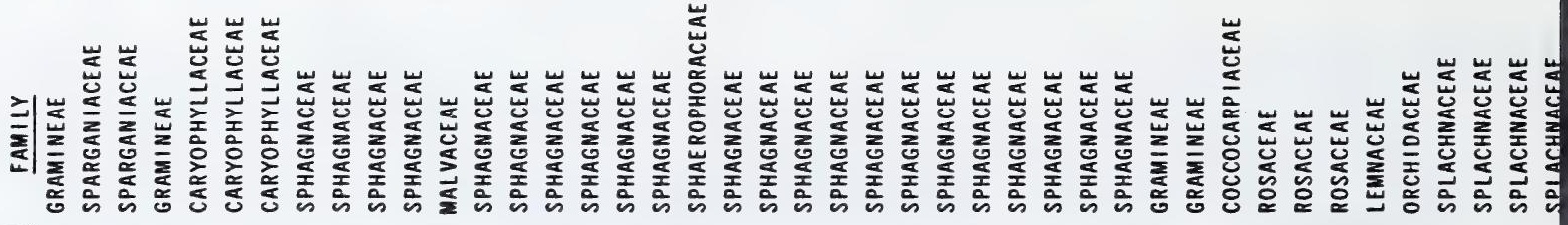

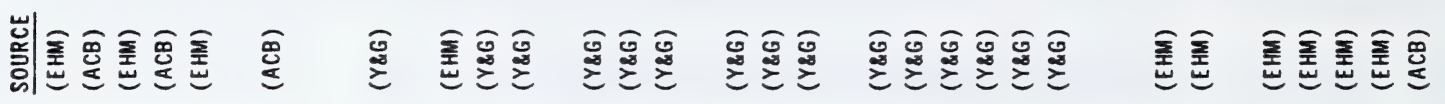

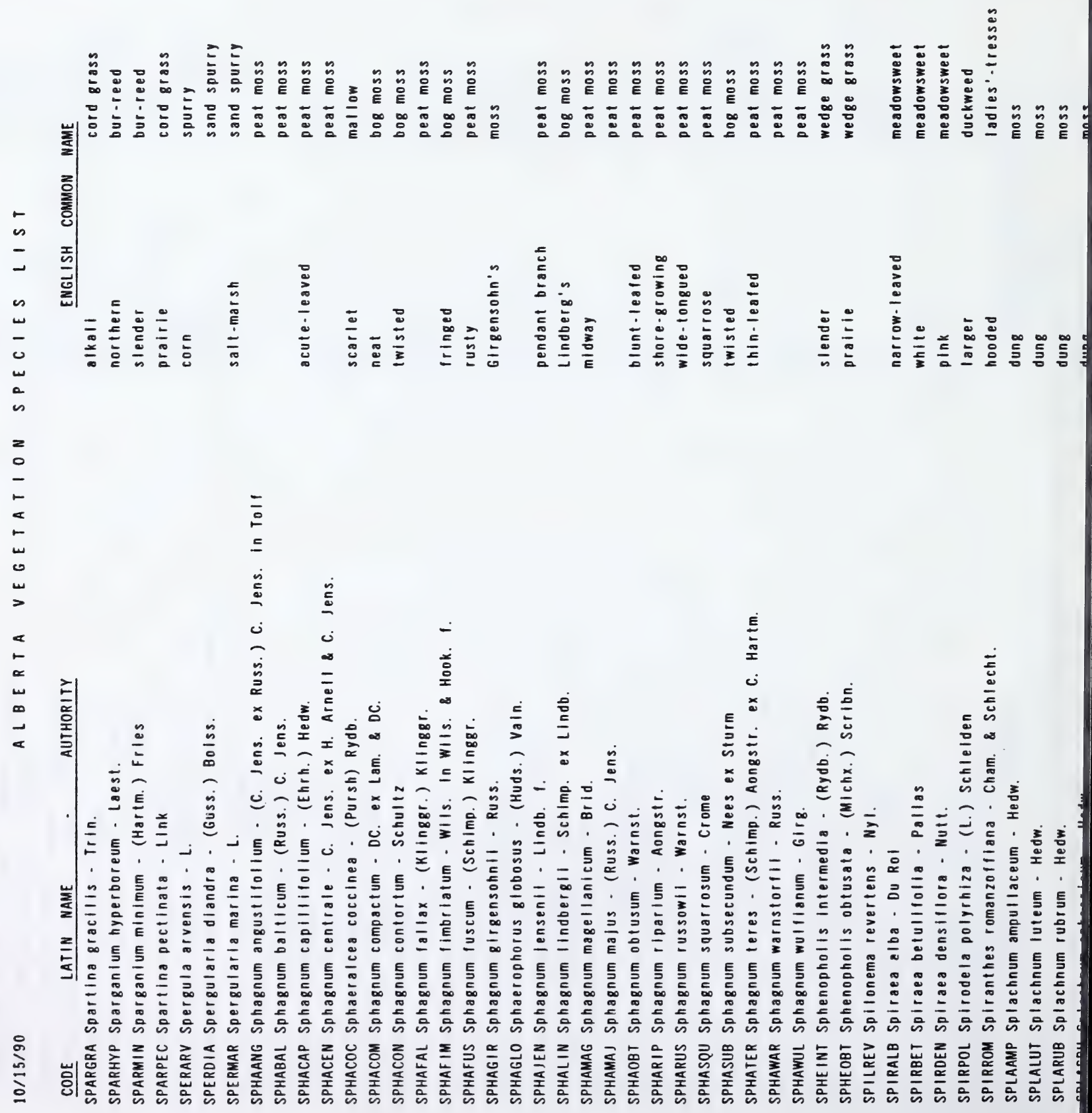




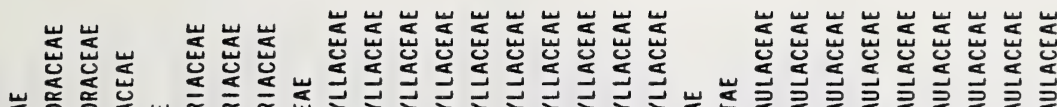

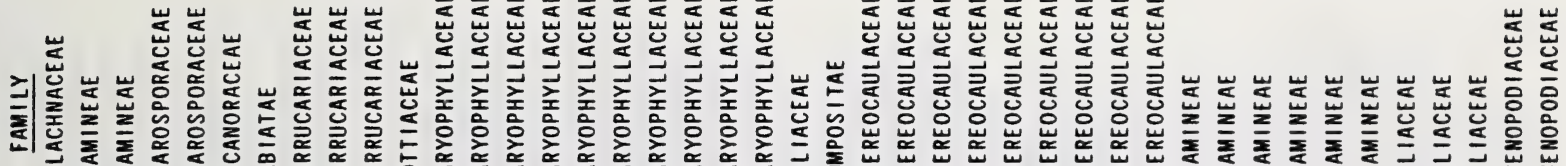

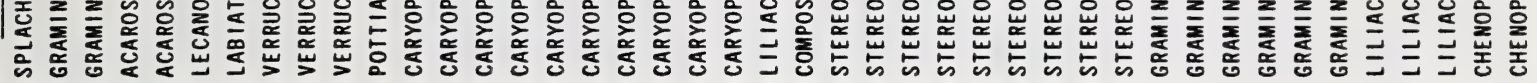

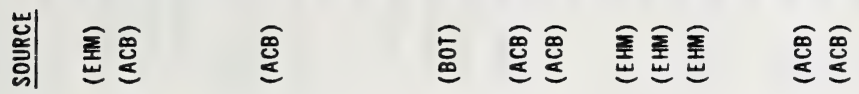

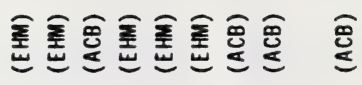

빟을

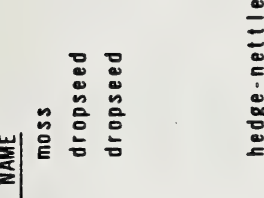

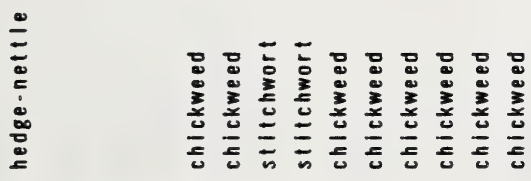

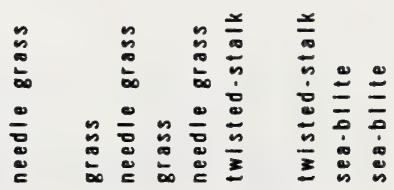

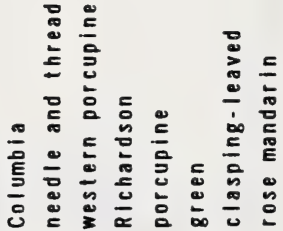

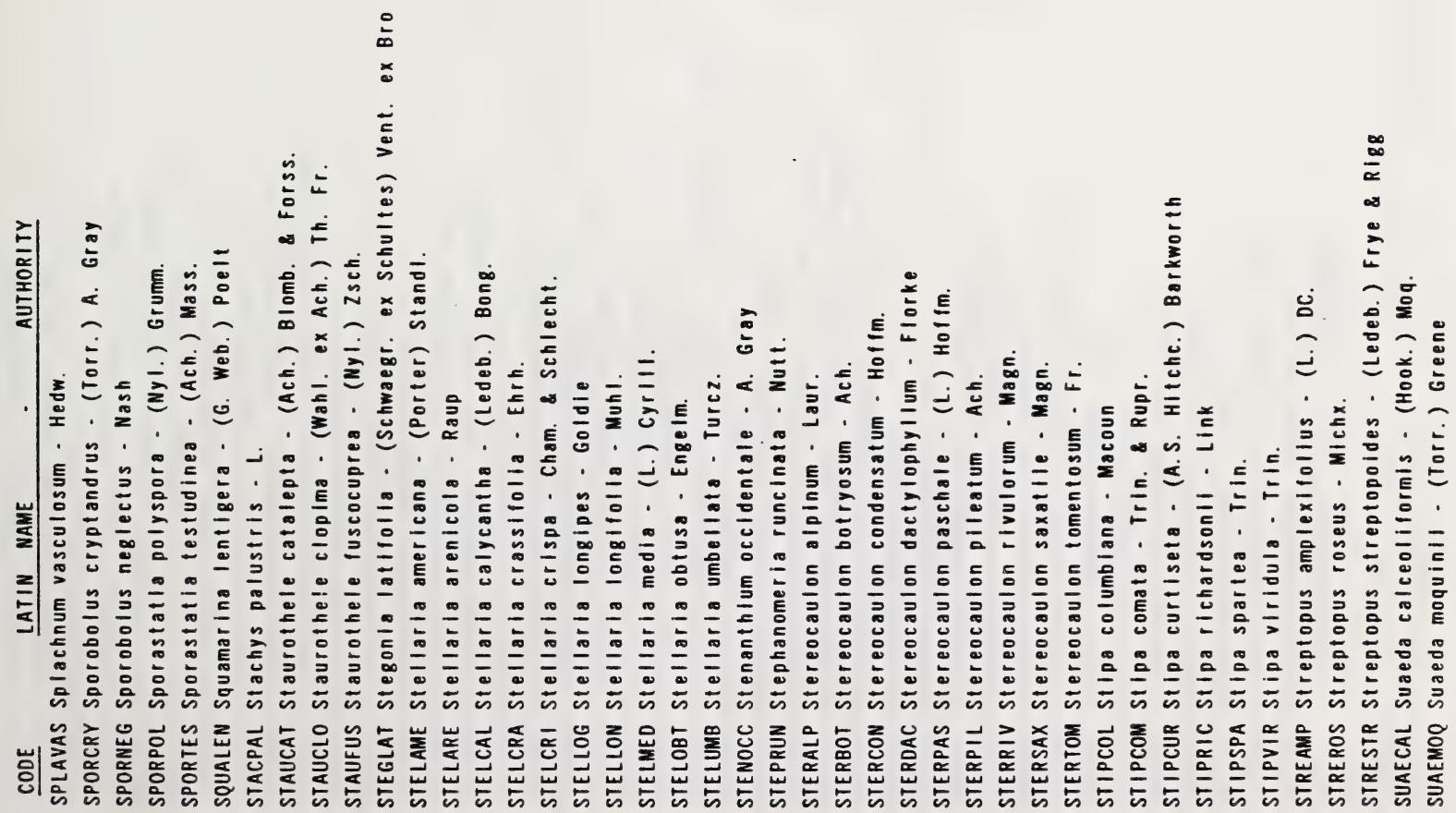




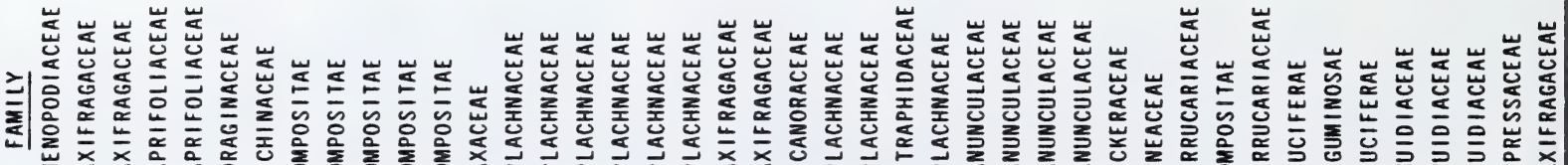

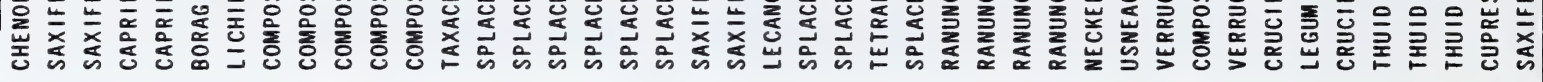

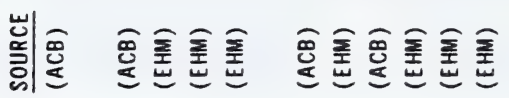

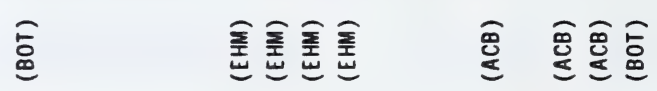

鋀要

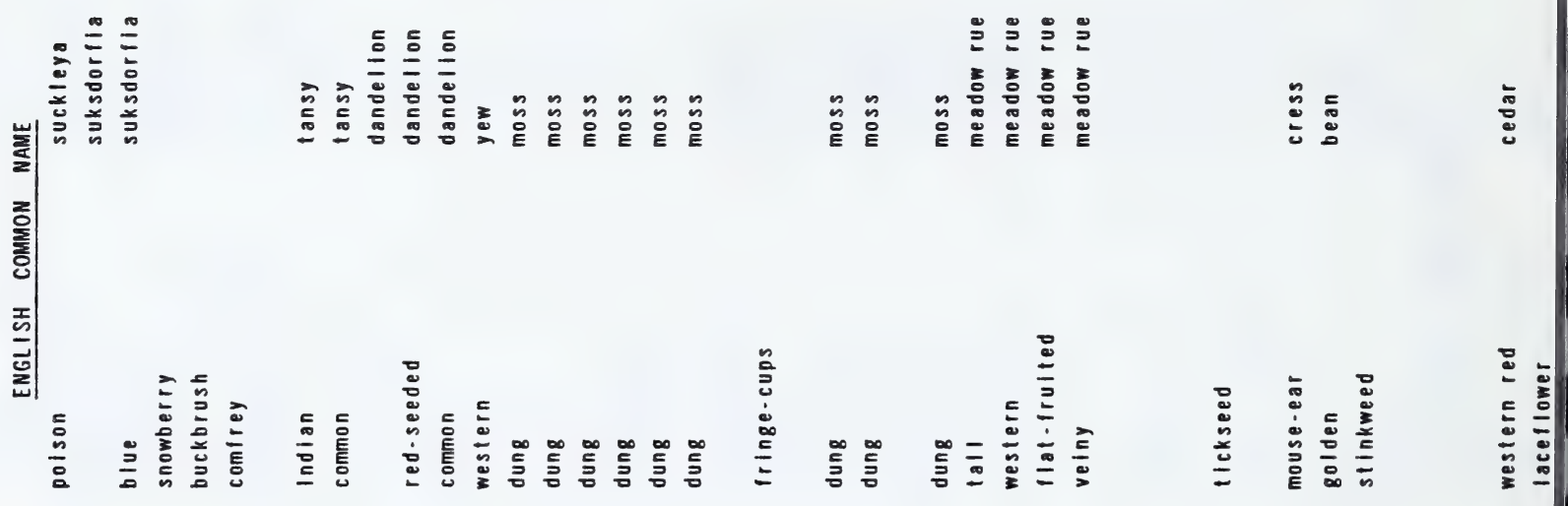

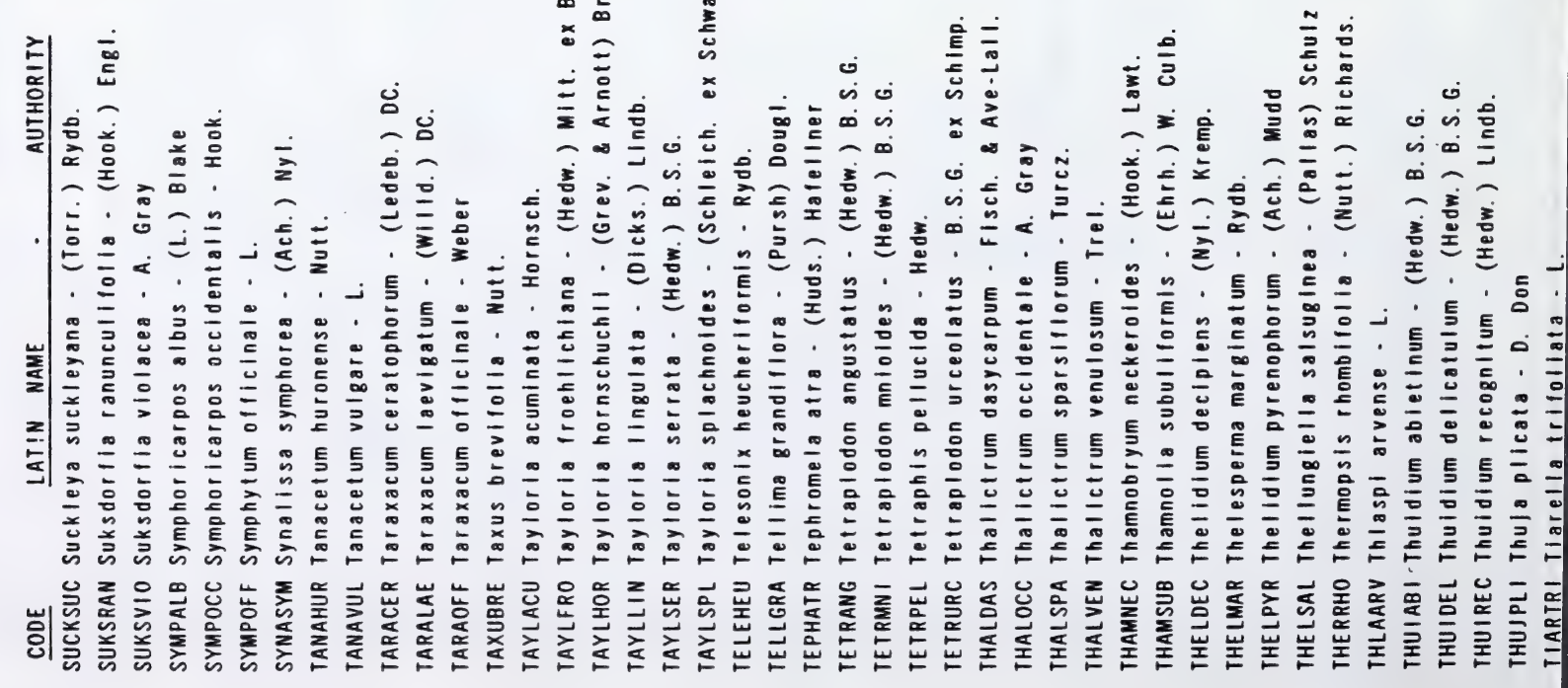


«

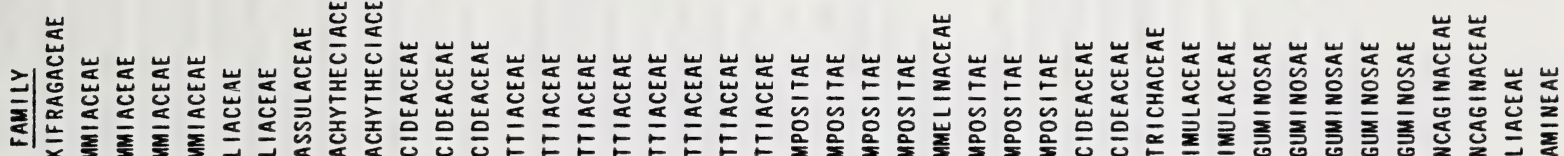

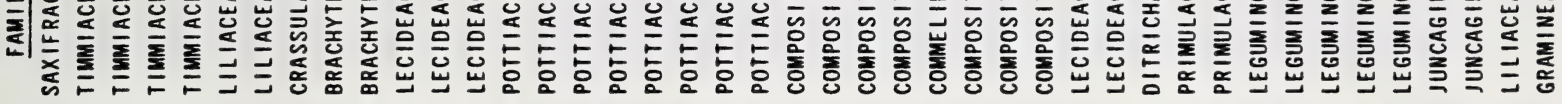

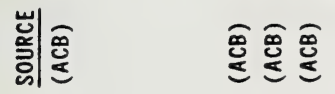

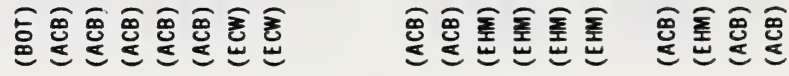
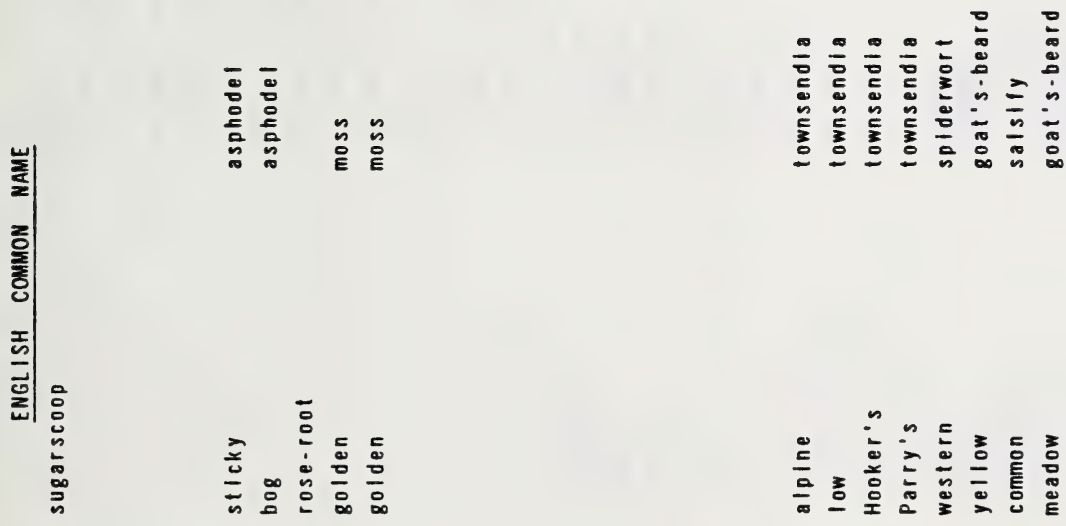

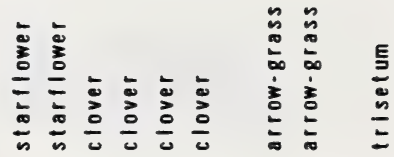

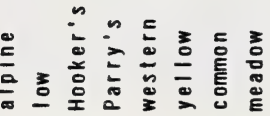

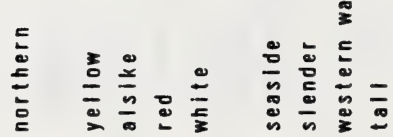

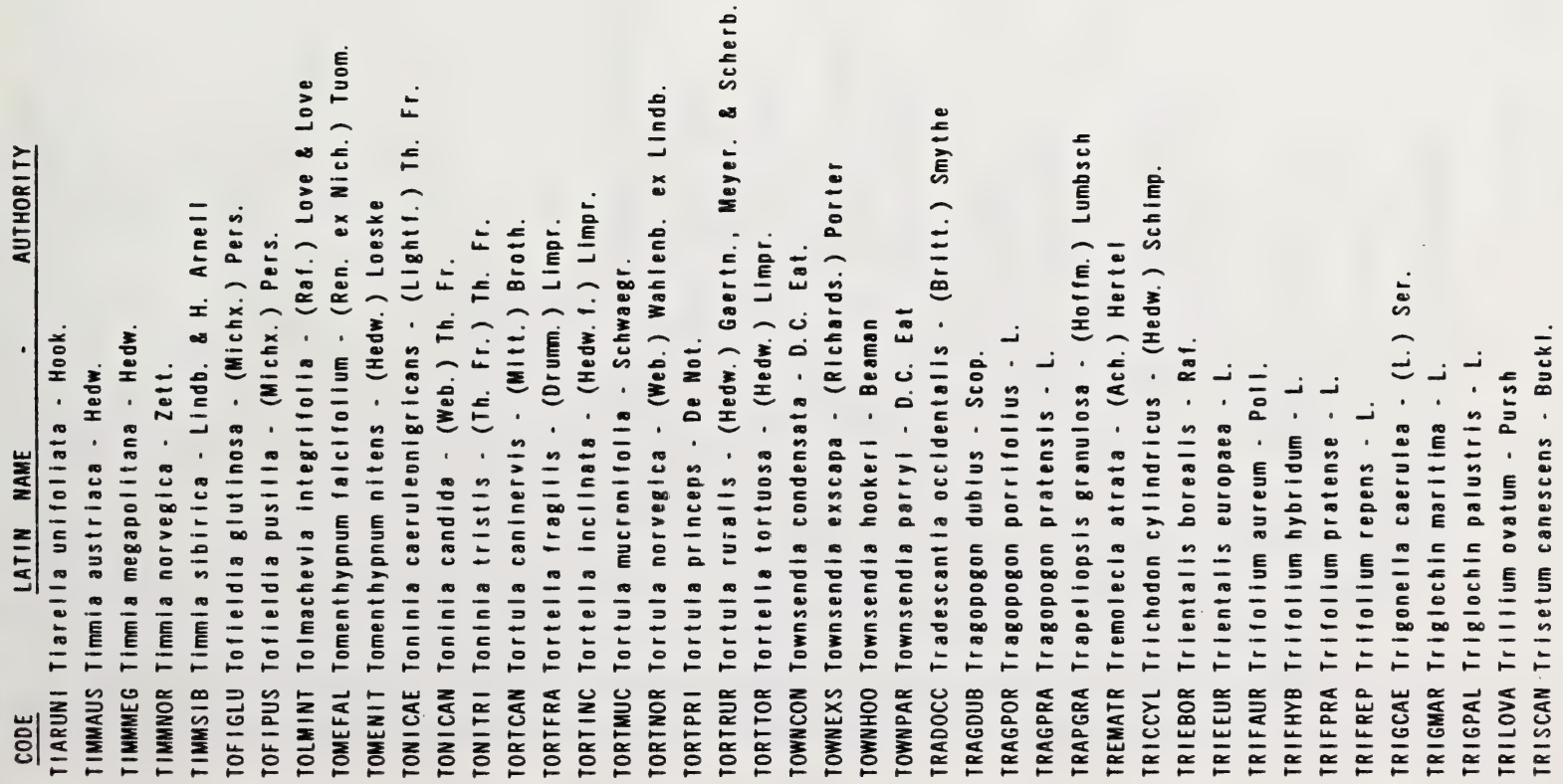




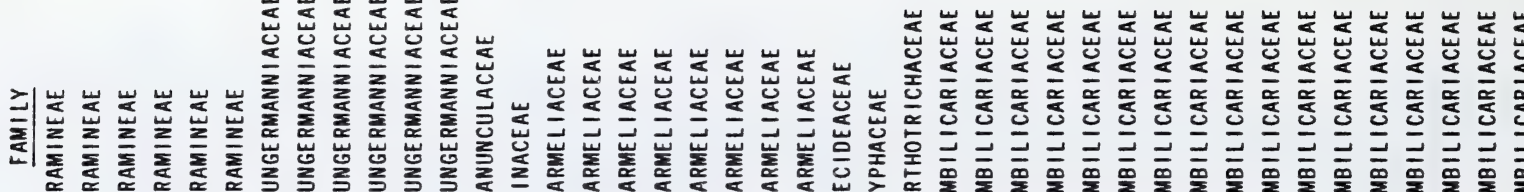

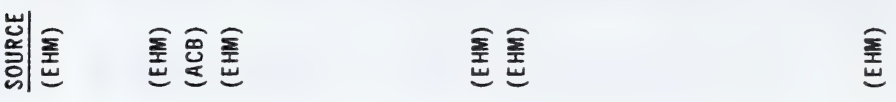

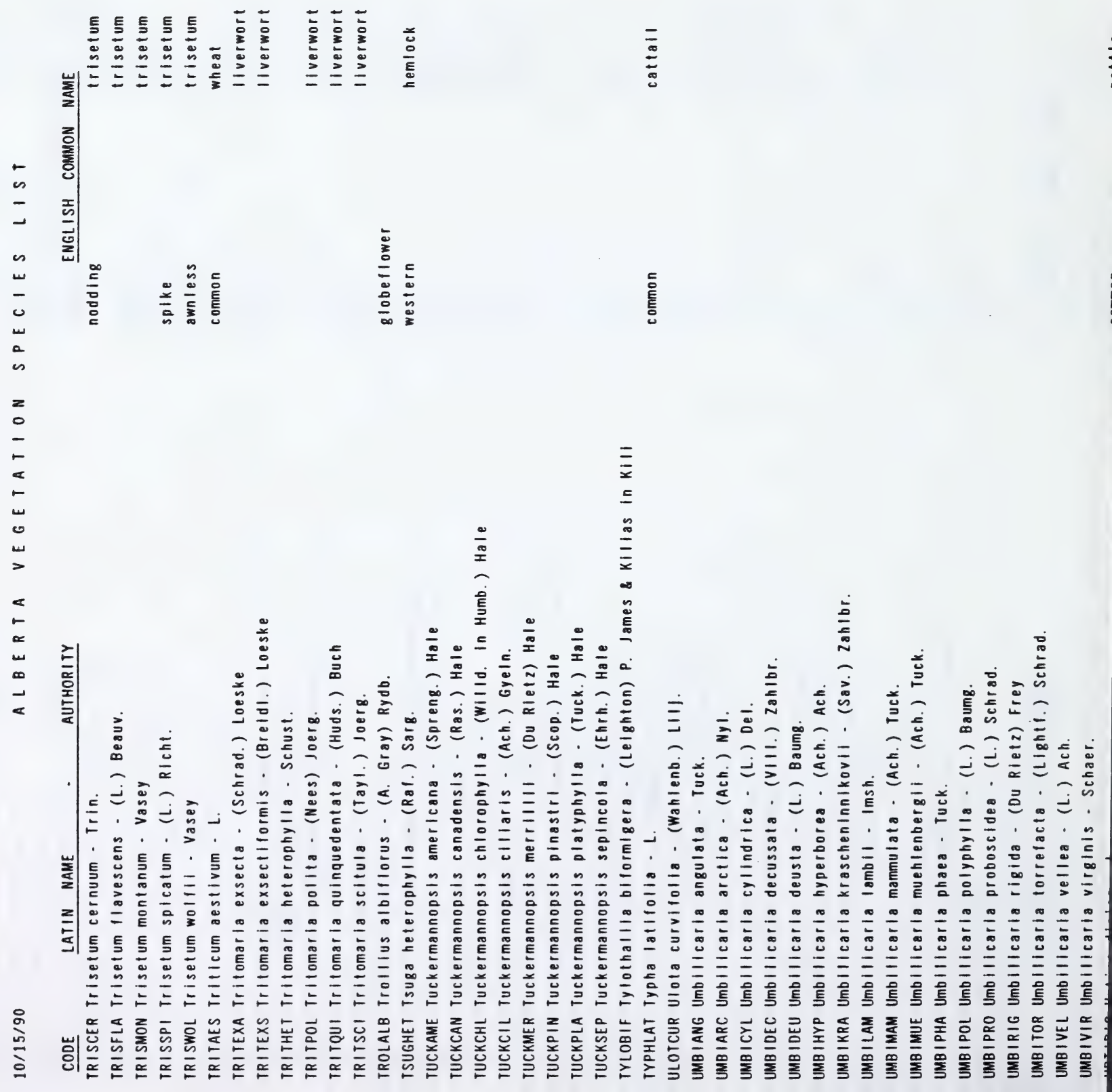




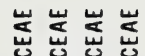

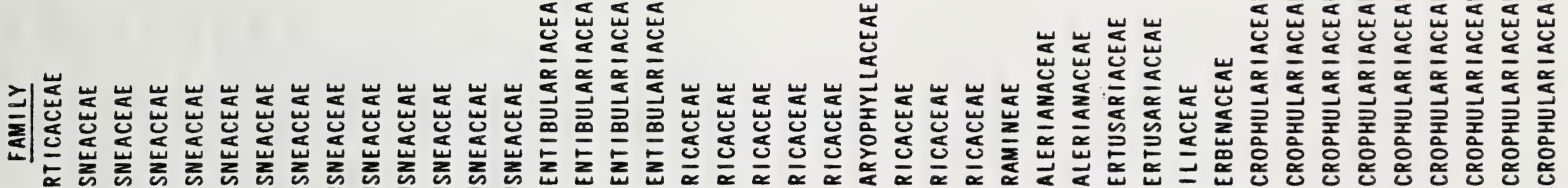

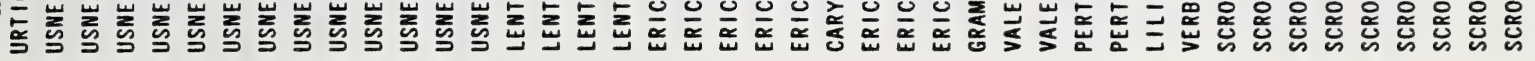
㓢部

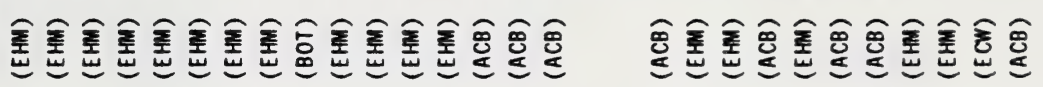

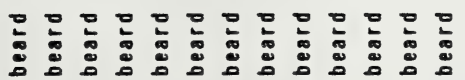

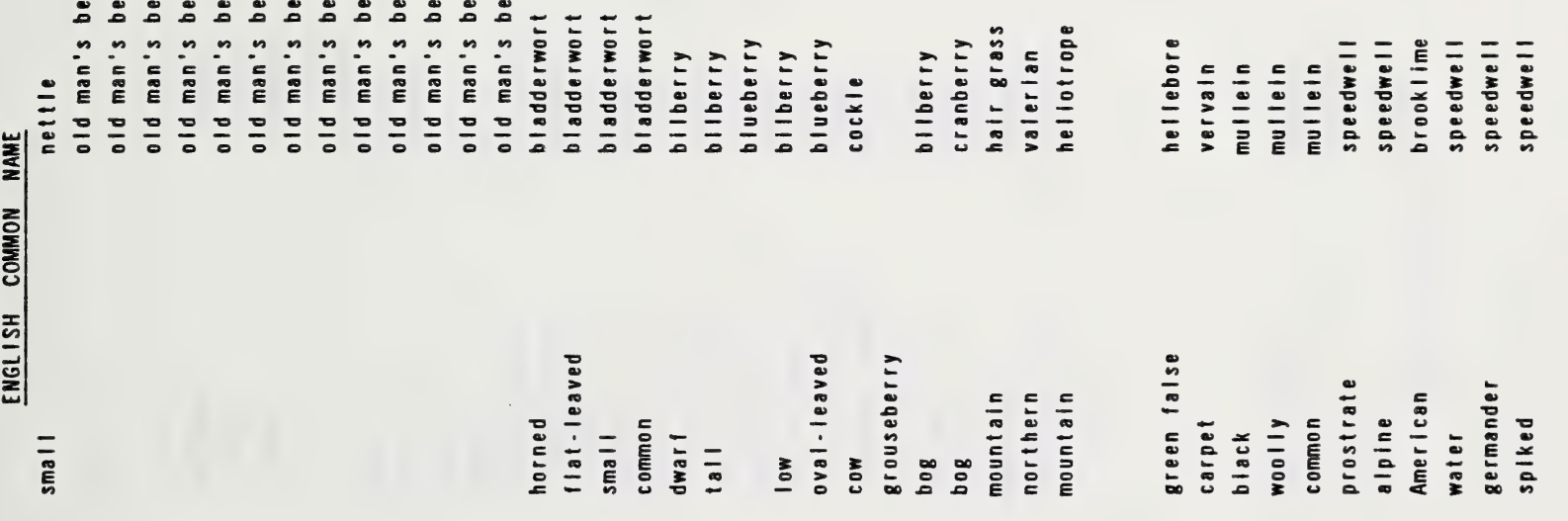

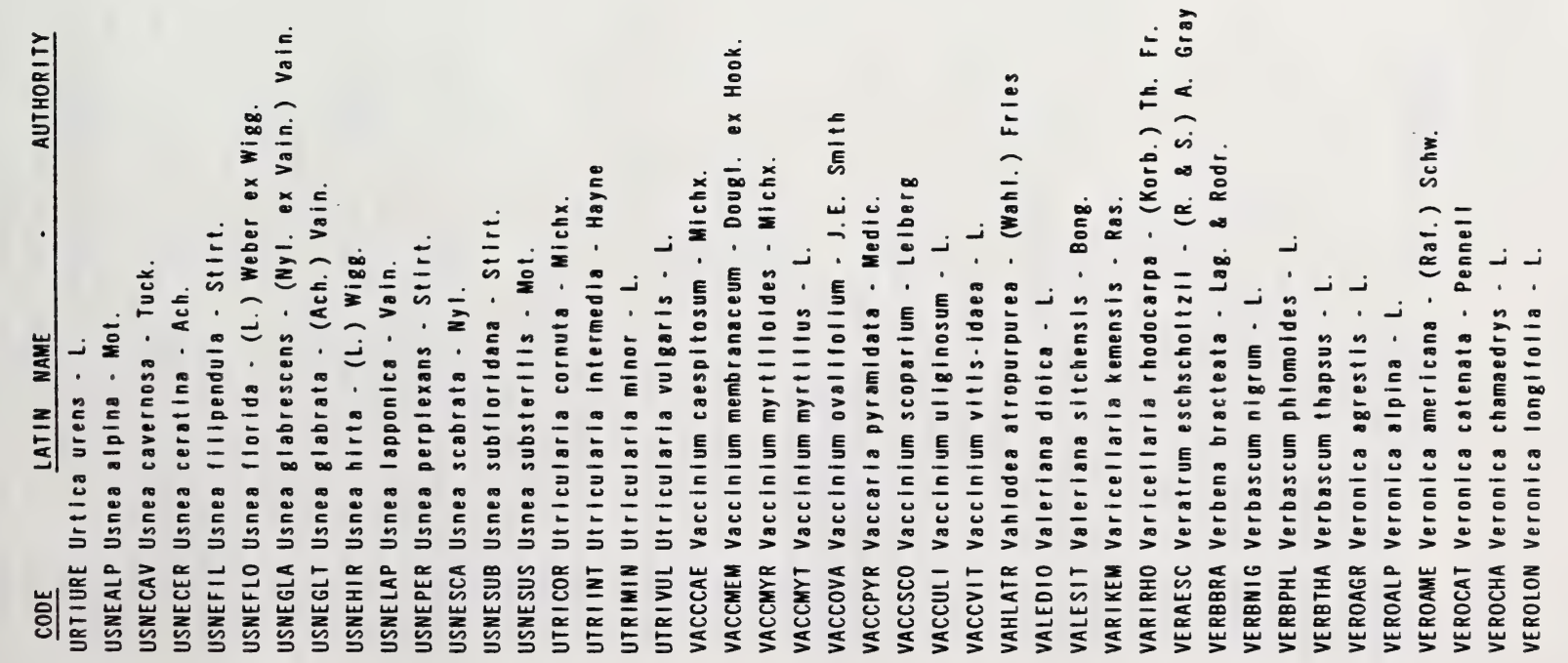


岁岸崖

岁岕岕岕岕岕岕岕岕岕岕

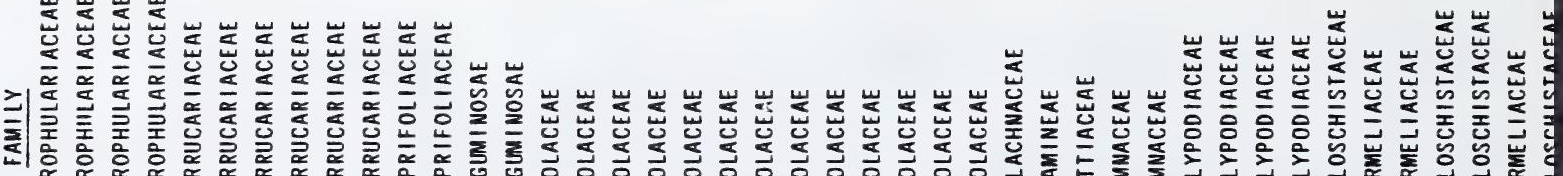

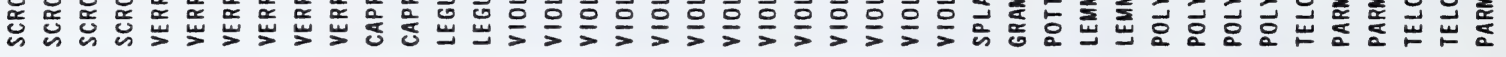

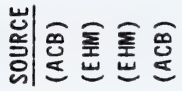

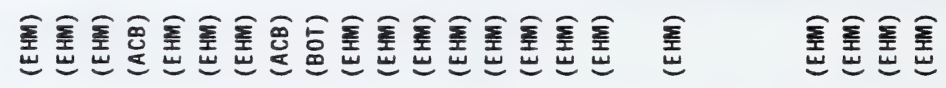

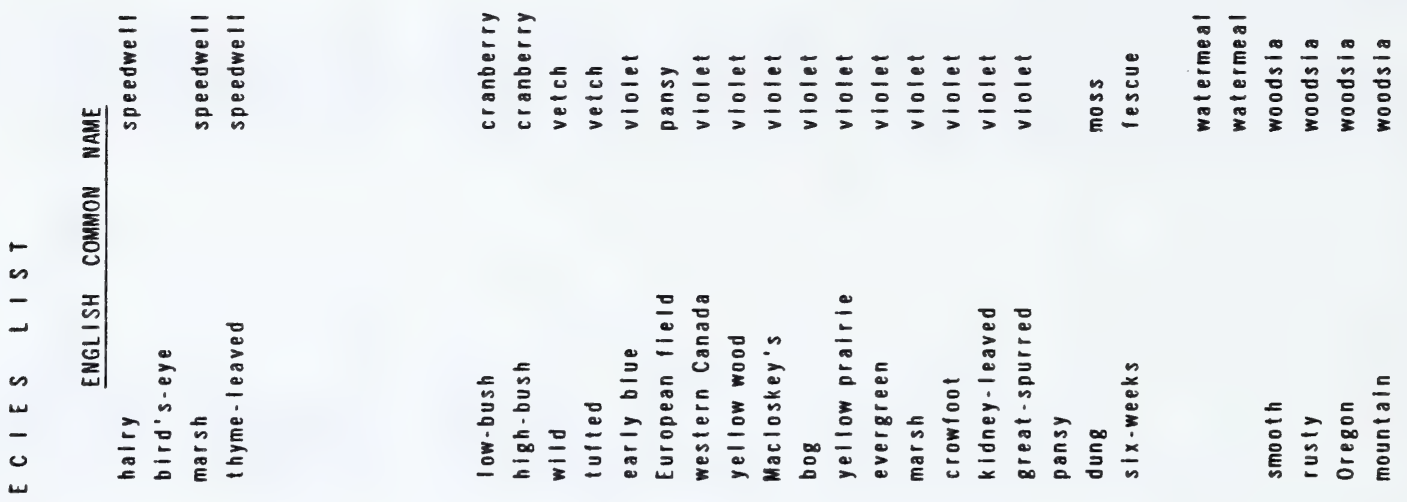




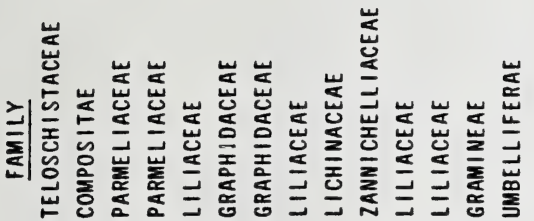

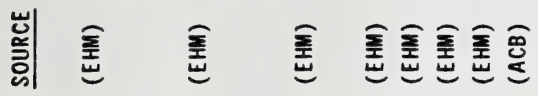

啧

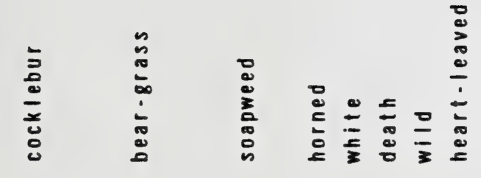

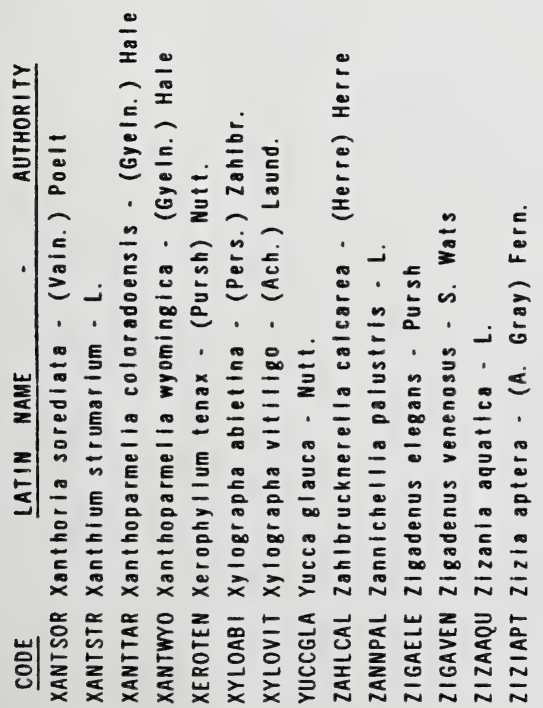




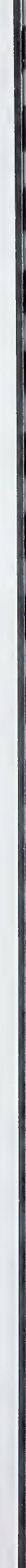





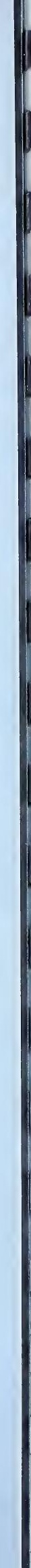


FAMILY LATIN NAME

ENGLISH COMMON NAME

CODE

CHECK

ACERACEAE

BE TULACEAE

BE TULACEAE

CUPRESSACEAE

PINACEAE

PINACEAE

PINACEAE

PINACEAE

PINACEAE

PINACEAE

PINACEAE

PINACEAE

PINACEAE

PINACEAE

PINACEAE

PINACEAE

PINACEAE

PINACEAE

PINACEAE

PINACEAE

PINACEAE

PINACEAE

ROSACEAE

SALICACEAE

SALI CACEAE

SALI CACEAE

SALICACEAE

SALICACEAE

Acer negundo
Betula neoalaskana
Betula papyrifera
Thuja plicata
Abies balsamea
Abies balsamea $x$ lasiocarpa
Abies lasiocarpa
Larix laricina
Larix lyallii
Larix occidentalis
Picea engelmannii
Picea engelmannii $x$ glauca
Picea glauca
Picea mariana
Pinus albicaulis
Pinus banksiana
Pinus contorta
Pinus contorta $x$ banksiana
Pinus flexilis
Pinus monticola
Pseudotsugamenziesii
Tsuga heterophylla
Sorbus aucuparia
Populus angustifolia
Populus balsamifera
Populus deltoides
Populus tremuloides
Populus x acuminata

Manitoba
Alaska
white
western red
balsam
balsam x alpine
alpine
tamarack
alpine
western
Engelmann
Engeimann $\times$ white
white
black
whitebark
jack
lodgepole
lodgepole $x$ jack
limber
western white
western
rowan tree
narrow-leaf
balsam
western
aspen
lance-leaf

maple
birch
birch
cedar
fir
fir
fir
larch
larch
spruce
spruce
spruce
spruce
pine
pine
pine
pine
pine
pine
Douglas-fir
hemlock
cottonwood
poplar
cottonwood
cottonwood

\begin{tabular}{|c|c|}
\hline S & ( \\
\hline ETUNEO & \\
\hline ETUPAP & \\
\hline THUSPLI & \\
\hline$A B \mid E B A L$ & \\
\hline ABIEBAS & \\
\hline$A B \mid E L A S$ & \\
\hline LARILAR & \\
\hline LARILYA & \\
\hline LARIOCC & \\
\hline PICEENG & \\
\hline PICEENE & \\
\hline PICEGLA & \\
\hline PICEMAR & \\
\hline PINUALB & \\
\hline PINUBAN & \\
\hline PINUCON & \\
\hline PINUCOB & \\
\hline PINUFLE & \\
\hline PINUMON & \\
\hline PSEUMEN & \\
\hline TSUGHET & \\
\hline SORBAUC & \\
\hline POPUANG & \\
\hline POPUBAL & \\
\hline POPUDEL & \\
\hline POPUTRE & \\
\hline POPUACU & \\
\hline
\end{tabular}


J 



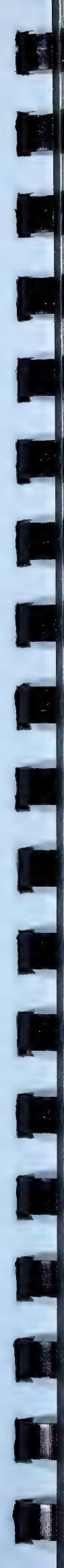


FAMILY

LATIN NAME

ENGLISH COMMON NAME

CODE CHECK

ACERACEAE

ANACARDIACEAE

ANACARDIACEAE

ARAL IACEAE

BERBERIDACEAE

BETULACEAE

BETULACEAE

BETULACEAE

BE TULACEAE

BETULACEAE

BETULACEAE

CAPRIFOLIACEAE

CAPRIFOLIACEAE

CAPRIFOLIACEAE

CAPRIFOLIACEAE

CAPRIFOLIACEAE

CAPRIFOL IACEAE

CAPRIFOLIACEAE

CAPRIFOLIACEAE

CAPRIFOLIACEAE

CAPRIFOLIACEAE

CAPRIFOLIACEAE

CELASTRACEAE

CHENOPODIACEAE

CHENOPODIACEAE

CHENOPODIACEAE

CHENOPODIACEAE

CISTACEAE

COMPOSITAE

COMPOSITAE

COMPOSITAE

CORNACEAE

CORNACEAE

CUPRESSACEAE

CUPRESSACEAE

CUPRESSACEAE

ELAEAGNACEAE

ELAEAGNACEAE

ELAEAGNACEAE

ELAEAGNACEAE

ELAEAGNACEAE

EMPETRACEAE

ERICACEAE

ERICACEAE

ERICACEAE

ERICACEAE

ERICACEAE

ERICACEAE

ERICACEAE

ERICACEAE

ERICACEAE

ERICACEAE

ERICACEAE

ERICACEAE
Acer glabrum

Rhus radicans

Rhus trilobata

Oplopanax horridum

Berberis repens

Alnus crispa

Alnus tenuifolia

Betula glandulosa

Betula occidentalis

Betula pumila

Corylus cornuta

Linnaea borealis

Lonicera caerulea

Lonicera dioica

Lonicera involucrata

Lonicera tartarica

Lonicera utahensis

Sambucus racemosa

Symphoricarpos albus

Symphoricarpos occidentalis

Viburnum edule

viburnum opulus

Pachistima myrsinites

Atriplex canescens

Atriplex nuttallii

Sarcobatus vermiculatus

Suada moquinii

Hudsonia tomentosa

Artemisia cana

Artemisia tridentata

Chrysothamnus nauseosus

Cornus canadensis

Cornus stolonifera

Juniperus communis

Juniperus horizontalis

Juniperus scopulorum

Elaeagnus angustifolia

Elaeagnus commutata

Hippophae rhamnoides

Shepherdia argentea

Shepherdia canadensis

Empetrum nigrum

Andromeda polifolia

Arctostaphylos rubra

Arctostaphylos uva-ursi

Cassiope mertensiana

Cassiope tetragona

Chamaedaphne calyculata

Gaultheria hispidula

Gaultheria humifusa

KaImia microphylla

Kalmia polifolia

Ledum glandulosum

Ledum groenlandicum

mountain
poison ivy
skunkbush
devil's-club
croeping
green
river
bog
water
dwarf
beaked
twinflower
fly
twining
bracted
tartarian
red
red

snowberry

buckbrush

low-bush

high-bush

mountain lover

Nuttall's

greasewood

sand

big

rabbitbrush

bunchberry

red-osier

ground

creeping

rocky mountain

Russian

silverberry

sea

thorny

Canada

crowberry

bog

alpine

common

western

white

leatherleaf

creeping

alpine

mountain

northern

glandular

common maple

ACERGLA （）

RHUSRAD ( )

RHUSTRI ( )

OPLOHOR ( )

BERBREP ( )

ALNUCRI ( )

ALNUTEN ( )

BETUGLA ( )

BETUOCC ( )

BETUPUM ( )

CORYCOR ( )

LINNBOR ( )

LONICAE ( )

LONIDIO ( )

LONIINV ( )

LONITAR ( )

LONIUTA ( )

SAMBRAC ( )

SYMPALB ( )

SYMPOCC ( )

VIBUEDU ( )

VIBUOPU ( )

PACHMYR ( )

ATRICAN ( )

ATRINUT ( )

SARCVER ( )

SUAEMOQ ( )

HUDSTOM ( )

ARTECAN ( )

ARTETRI ( )

CHRYNAU ( )

CORNCAN ( )

CORNSTO ( )

JUNICOM ( )

JUNIHOR ( )

JUNCSCO ( )

ELAEANG ( )

ELAECOM ( )

HIPPRHA ( )

SHEPARG ( )

SHEPCAN ( )

EMPENIG ( )

ANDRPOL ( )

ARCTRUB ( )

ARCTUVA ( )

CASSMER ( )

CASSTET ( )

CHAMCAL ( )

GAULHIS ( )

GAULHUM ( )

KALMMIC ( )

KALMPOL ( )

LEDUGLA ( )

LEDUGRO ( ) 


\section{ERICACEAE \\ ERI CACEAE \\ ERICACEAE \\ ERICACEAE \\ ERICACEAE \\ ERICACEAE \\ ERICACEAE \\ ERICACEAE \\ ERICACEAE \\ ERICACEAE \\ ERICACEAE \\ ERICACEAE \\ ERICACEAE \\ ERICACEAE \\ ERICACEAE \\ ERICACEAE \\ ERICACEAE \\ ERICACEAE}

GROSSULARIACEAE

GROSSULARIACEAE

GROSSULARIACEAE

GROSSULARIACEAE

GROSSULARIACEAE

GROSSULARIACEAE

GROSSULARIACEAE

GROSSULARIACEAE

GROSSULARIACEAE

GROSSULARIACEAE

GROSSULARIACEAE

HYDRANGEACEAE

LEGUMINOSAE

MALVACEAE

MYRICACEAE

POLYGONACEAE

PYROLACEAE

RANUNCULACEAE

RANUNCULACEAE

RANUNCULACEAE

RHAMNACEAE

RHAMNACEAE

RHAMNACEAE

ROSACEAE

ROSACEAE

ROSACEAE

ROSACEAE

ROSACEAE

ROSACEAE

ROSACEAE

ROSACEAE

ROSACEAE

ROSACEAE

ROSACEAE

ROSACEAE

ROSACEAE
Ledum palustre

Loiseleuria procumbens

Menziesia ferruginea

Oxycoccus microcarpus

Oxycoccus quadripetalus

Phyllodoce empetriformis

Phyllodoce glanduliflora

Phyllodoce $x$ intermedia

Rhododendron albiflorum

Rhododendron Iapponicum

Vaccinium caespitosum

Vaccinium membranaceum

Vaccinium myrtilloides

Vaccinium myrtillus

Vaccinium ovalifolium

Vaccinium scoparium

Vaccinium uliginosum

Vaccinium vitis-idaea

Ribes americanum

Ribes aureum

Ribes glandulosum

Ribes hirtellum

Ribes hudsonianum

Ribes inerme

Ribes lacustre

Ribes laxiflorum

Ribes oxyacanthoides

Ribes triste

Ribes viscosissimum

Philadelphus lewisi

Caragana arborescens

Sphaeralcea coccinea

Myrica gale

Eriogonum umbellatum

Chimaphila umbellata

Clematis ligusticifolia

Clematis occidentalis

Clematis tangutica

Ceanothus velutinus

Rhamnus alnifolia

Rhamnus catharticus

Amelanchier alnifolia

Crataegus douglasii

Crataegus rotundifolia

Dryas drummondii

Dryas integrifolia

Dryas octopetala

Luetkea pectinata

Physocarpus malvaceus

Potentilla fruticosa

Prunus pensyivanica

Prunus virginiana

Rosa acicularis

Rosa arkansana

northern
alpine
false
small
small
red
yellow

white-flowered

Lapland

dwar $f$

tall

low

oval-leaved

grouseberry

bog

bog

wild black

golden

skunk

wild

northern black

mountain

bristly black

mountain

wild

wild red

sticky

moçk orange

common

scarlet

sweet

prince's-pine

western

purple

yel low

snowbrush

alder-leaved

common

saskatoon

Douglas

round - leaved

yellow

white

white

partridgefoot

mallow-leaved

shrubby

pin

choke

prickly

prairie
Labrador tea

azalea

azalea

bog cranberry

bog cranberry

heather

heather

heather

rhododendron

rose-bay

bilberry

bilberry

blueberry

bilberry

blueberry

bilberry

cranberry

currant

currant

currant

gooseberry

currant

gooseberry

currant

currant

$800 s e b e r r y$

currant

currant

caragana

mallow

gale

umbre I laplant

clemat is

clemat is

clemat is

buckthorn

buckthorn

hawthorn

hawthorn

mountain avens

mountain avens

mountain avens

ninebark

cinquefoil

cherry

cherry

rose

rose
LEDUPAL ( )

LOISPRO ( )

MENZFER ( )

OXYCMIC ( )

OXYCQUA ( )

PHYLEMP ( )

PHYLGLA ( )

PHYLINT ( )

RHODALB ( )

RHODLAP ( )

VACCCAE ( )

VACCMEM ( )

VACCMYR ( )

VACCMYT ( )

VACCOVA ( )

VACCSCO ( )

VACCULI ( )

VACCVIT ( )

RIBEAME ( )

RIBEAUR ( )

RIBEGLA ( )

RIBEHIR ( )

RIBEHUD ( )

RIBEINE ( )

RIBELAC ( )

RIBELAX ( )

RIBEOXY ( )

RIBETRI ( )

RIBEVIS ( )

PHILLEW ( )

CARAARB ( )

SPHACOC ( )

MYRIGAL ( )

ERIOUMB ( )

CHIMUMB ( )

CLEMLIG ( )

CLEMOCC ( )

CLEMTAN ( )

CEANVEL ( )

RHAMALN ( )

RHAMCAT ( )

AMELALN ( )

CRATDOU ( )

CRATROT ( )

DRYADRU ( )

DRYAINT ( )

DRYAOCT ( )

LUETPEC ( )

PHYSMAL ( )

POTEFRU ( )

PRUNPEN ( )

PRUNVIR ( )

ROSAACI ( )

ROSAARK ( ) 
ROSACEAE

ROSACEAE

ROSACEAE

ROSACEAE

ROSACEAE

ROSACEAE

ROSACEAE

ROSACEAE

ROSACEAE

ROSACEAE

ROSACEAE

ROSACEAE

ROSACEAE

ROSACEAE

SALICACEAE

SALI CACEAE

SALICACEAE

SALICACEAE

SALICACEAE

SALICACEAE

SALI CACEAE

SALI CACEAE

SALI CACEAE

SALICACEAE

SALICACEAE

SAL I CACEAE

SALICACEAE

SALICACEAE

SAL I CACEAE

SAL I CACEAE

SALICACEAE

SAL I CACEAE

SAL I CACEAE

SAL I CACEAE

SAL I CACEAE

SAL I CACEAE

SAL I CACEAE

SAL I CACEAE

SAL I CACEAE

SAL I CACEAE

SALI CACEAE

SAL I CACEAE

SALI CACEAE

SALI CACEAE

SALICACEAE

SAL I CACEAE

SALI CACEAE

SAL I CACEAE

SALI CACEAE

SALICACEAE

SOLANACEAE

TAXACEAE
Rosa woodsii

Rubus arcticus

Rubus chamaemorus

Rubus idaeus

Rubus parviflorus

Rubus pedatus

Rubus pubescens

Rubus $x$ paracaulis

Sibbaldia procumbens

Sorbus scopulina

Sorbus sitchensis

Spiraea alba

Spiraea betulifolia

Spiraea densiflora

Salix alaxensis

Salix amygdaloides

Salix arbusculoides

Salix arctica

Salix athabascensis

Salix barclayi

Salix barrattiana

Salix bebbiana

Salix boothii

Salix brachycarpa

Salix candida

Salix commutata

Salix discolor

Salix drummondiana

Salix exigua

Salix farriae

Salix glauca

Salix Ianata

Salix lucida

Salix lutea

Salix maccalliana

Salix melanopsis

Salix myrtillifolia

Salix pedicellaris

Salix petiolaris

Salix planifolia

Salix prolixa

Salix pseudomonticola

Salix pyrifolia

Salix raupii

Salix reticulata

Salix scouleriana

Salix serissima

Salix sitchensis

Salix stolonifera

Salix vestita

Lycium halimifolium

Taxus brevifolia commo

cloudberry

wild red

thimbleberry

dwar $f$

dewberry

dwarf

sibbaldia

western

narrow-leaved

white

pink

Alaska

peach-leaved

shrubby

arctic

Athabasca

Barclay's

Barratt's

beaked

Booth's

short-capsuled

hoary

changeable

pussy

satin

sandbar

Farr's

smooth

woolly

shining

yellow

velvet-fruited

myrtle-leaved

bog

basket

flat-leaved

Mackenzie's

false mountain

bals am

Raup's

snow

Scouler's

aut umn

Sitka

rock

matrimony

western rose
raspberry
raspberry
bramble
raspberry

mountain ash

mountain ash

meadowsweet

meadowswe $t$

meadowswe t

willow

willow

willow

willow

willow

willow

willow

willow

willow

willow

willow

willow

willow

willow

willow

willow

willow

willow

willow

willow

willow

willow

willow

willow

willow

willow

willow

willow

willow

willow

willow

willow

willow

willow

willow

willow

vine

yew
ROSAWOO ( )

RUBUARC ( )

RUBUCHA ( )

RUBUIDA ( )

RUBUPAR ( )

RUBUPED ( )

RUBUPUB ( )

RUBUPAA ( )

SIBBPRO ( )

SORBSCO ( )

SORBSIT ( )

SPIRALB ( )

SPIRBET ( )

SPIRDEN ( )

SALIALA ( )

SALIAMY ( )

SALIARB ( )

SALIARC ( )

SALIATH ( )

SALIBAR ( )

SALIBAA ( )

SALIBEB ( )

SALIBOO ( )

SALIBRA ( )

SALICAN ( )

SALICOM ( )

SALIDIS ()

SALIDRU ( )

SALIEXI ( )

SALIFAR ( )

SALIGLA ( )

SALILAN ( )

SALILUC ( )

SALILUT ( )

SALIMAC ( )

SALIMEL ( )

SALIMYR ( )

SALIPED ( )

SALIPET ( )

SALIPLA ( )

SALIPRO ( )

SALIPSE ( )

SALIPYR ( )

SALIRAU ( )

SALIRET ( )

SALISCO ( )

SALISER ( )

SALISIT ( )

SALISTO ()

SALIVES ( )

LYCIHAL ( )

TAXUBRE ( ) 


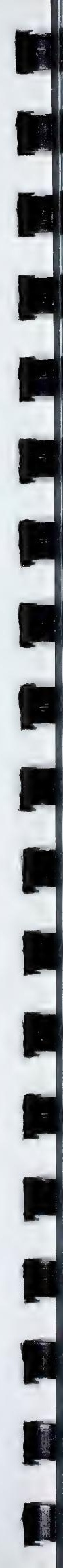





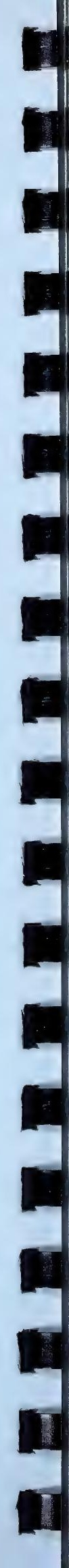


ADOXACEAE

AL I SMATACEAE

ALI SMATACEAE

ALI SMATACEAE

AL I SMATACEAE

AMARANTHACEAE

AMARANTHACEAE

AMARANTHACEAE

AMARANTHACEAE

AMARANTHACEAE

APOCYNACEAE

APOCYNACEAE

APOCYNACEAE

ARACEAE

ARACEAE

ARAL IACEAE

ASCLEP I ADACEAE

ASCLEPIADACEAE

ASCLEP IADACEAE

BALSAMINACEAE

BALSAMINACEAE

BORAGINACEAE

BORAGINACEAE

BORAGINACEAE

BORAGINACEAE

BORAGINACEAE

BORAGINACEAE

BORAGINACEAE

BORAG INACEAE

BORAGINACEAE

BORAGINACEAE

BORAGINACEAE

BORAGINACEAE

BORAGINACEAE

BORAGINACEAE

BORAGINACEAE

BORAGINACEAE

BORAGINACEAE

BORAGINACEAE

BORAGINACEAE

BORAGINACEAE

BORAGINACEAE

BORAGINACEAE

BORAGINACEAE

BORAGINACEAE

BORAGINACEAE

BORAGINACEAE

BORAGINACEAE

BORAGINACEAE

BORAGINACEAE

BORAGINACEAE

CACTACEAE

CACTACEAE

CACTACEAE
Adoxa moschatellina

Alisma gramineum

Alisma plantago-aquatica

Sagittaria cuneata

Sagittaria latifolia

Amaranthus albus

Amaranthus californicus

Amaranthus graecizans

Amaranthus powellii

Amaranthus retroflexus

Apocynum androsaemifolium

Apocynum cannabinum

Apocynum $x$ medium

Acorus americanus

Calla palustris

Aralia nudicaulis

Asclepias ovalifolia

Asclepias speciosa

Asclepias viridiflora

Impatiens capensis

Impatiens noli-tangere

Amsinckia menziesii

Asperugo procumbens

Borago officinalis

Cryptantha fendleri

Cryptantha macounii

Cryptantha minima

Cryptantha nubigena

Cynoglossum boreale

Cynoglossum officinale

Echium vulgare

Hackelia americana

Hackelia floribunda

Hackelia jessicae

Heliotropium curassavicum

Lappula occidentalis

Lappula squarrosa

Li thospermum incisum

Lithospermum ruderale

Lycopsis arvensis

Mertensia lanceolata

Mertensia longiflora

Mertensia paniculata

Myosotis alpestris

Myosotis arvensis

Myosotis laxa

Myosotis micrantha

Nonea vesicaria

Onosmodium molle

Plagiobothrys scouleri

Symphytum officinale

Coryphantha vivipara

Opuntia fragilis

Opuntia polyacantha moschatel

narrow-leaved

broad-leaved

arum-leaved

broad-leaved

tumb lewe ed

prostrate

red-root

spreading

Indian

intermediate

sweet flag

water

wild

low

showy

green

spotted

western

fiddle-neck

madwort

oxtongue

Fendler's

Macoun's

small

clustered

wild

hound's-tongue

viper's-bugloss

nodding

large-flowered

Jessica's

spatulate-leaved

narrow-l eaved

woolly

small

lance-leaved

large-flowered

tall

alpine

field

smal I

red

western false

Scouler's

comfrey

cushion

brittle
ADOXMOS ( )

ALISGRA ( )

ALISPLA ( )

SAGICUN ( )

SAGILAT ( )

AMARALB ( )

AMARCAL ( )

AMARGRA ( )

AMARPOW ( )

AMARRET ( )

APOCAND ( )

APOCCAN ( )

APOCMED ( )

ACORAME ( )

CALLPAL ( )

ARALNUD ( )

ASCLOVA ( )

ASCLSPE ( )

ASCLVIR ( )

IMPACAP ( )

IMPANOL ( )

AMSIMEN ( )

ASPEPRO ( )

BORAOFF ( )

CRYPFEN ( )

CRYPMAC ( )

CRYPMIN ( )

CRYPNUB ( )

CYNOBOR ( )

CYNOOFF ( )

ECHIVUL ( )

HACKAME ( )

HACKFLO ( )

HACKJES ( )

HELICUR ( )

LAPPOCC ( )

LAPPSQU ( )

LITHINC ( )

LITHRUD ( )

LYCOARV ( )

MERTLAN ( )

MERTLON ( )

MERTPAN ( )

MYOSALP ( )

MYOSARV ( )

MYOSLAX ( )

MYOSMIC ( )

NONEVES ( )

ONOSMOL ( )

PLAGSCO ( )

SYMPOFF ( )

CORYVIV ()

OPUNFRA ( )

OPUNPOL ( ) 
CALLITRICHACEAE CALLITRICHACEAE CAMPANULACEAE CAMPANULACEAE CAMPANULACEAE CAMPANULACEAE CANNAB INACEAE CANNAB INACEAE CAPPARIDACEAE CAPPARIDACEAE CARYOPHYLLACEAE CARYOPHYLLACEAE CARYOPHYLLACEAE CARYOPHYLLACEAE CARYOPHYLLACEAE CARYOPHYLLACEAE CARYOPHYLLACEAE CARYOPHYLLACEAE CARYOPHYLLACEAE CARYOPHYLLACEAE CARYOPHYLLACEAE CARYOPHYLLACEAE CARYOPHYLLACEAE CARYOPHYLLACEAE CARYOPHYLLACEAE CARYOPHYLLACEAE CARYOPHYLLACEAE CARYOPHYLLACEAE CARYOPHYLLACEAE CARYOPHYLLACEAE CARYOPHYLLACEAE CARYOPHYLLACEAE CARYOPHYLLACEAE CARYOPHYLLACEAE CARYOPHYLLACEAE CARYOPHYLLACEAE CARYOPHYLLACEAE CARYOPHYLLACEAE CARYOPHYLLACEAE CARYOPHYLLACEAE CARYOPHYLLACEAE CARYOPHYLLACEAE CARYOPHYLLACEAE CARYOPHYLLACEAE CARYOPHYLLACEAE CARYOPHYLLACEAE CARYOPHYLLACEAE CARYOPHYLLACEAE CARYOPHYLLACEAE CARYOPHYLLACEAE CARYOPHYLLACEAE CARYOPHYLLACEAE CARYOPHYLLACEAE CARYOPHYLLACEAE

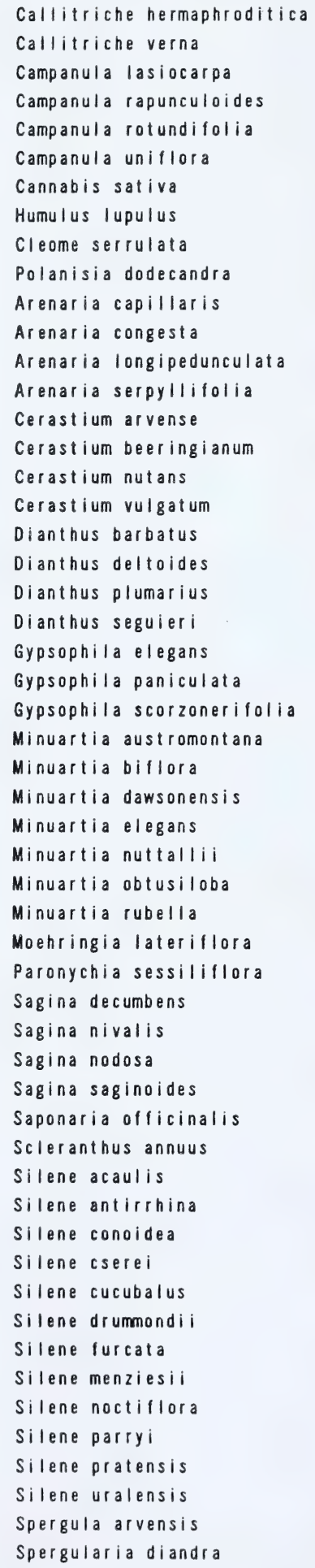

northern
vernal
Alaska
garden
alpine
common
bee plant
clammyweed
linear-leaved
rocky-ground
thyme-leaved
field
long-stalked mouse-eared
mouse-eared
sweet
maiden
garden

water-starmort

campion
catchfly
catchfly
catchfly
campion
cockle
catchfly
catchfly
catchfly
campion
cockle
cockle
spurry
sand spurry

CALLHER ( )

CALLVER ( )

CAMPLAS ( )

CAMPRAP ( )

CAMPROT ( )

CAMPUNI ( )

CANNSAT ( )

HUMULUP ( )

CLEOSER ( )

POLADOD ( )

ARENCAP ( )

ARENCON ( )

ARENLON ( )

ARENSER ( )

CERAARV ( )

CERABEE ( )

CERANUT ( )

CERAVUL ( )

DIANBAR ( )

DIANDEL ( )

DIANPLU ( )

DIANSEG ( )

GYPSELE ( )

GYPSPAN ( )

GYPSSCO ( )

MINUAUS ( )

MINUBIF ( )

MINUDAW ( )

MINUELE ( )

MINUNUT ( )

MINUOBT ( )

MINURUB ( )

MOEHLAT ( )

PAROSES ( )

SAGIDEC ( )

SAGINIV ( )

SAGINOD ( )

SAGISAG ( )

SAPOOFF ( )

SCLEANN ( )

SILEACA ( )

SILEANT ( )

SILECON ( )

SILECSE ( )

SILECUC ( )

SILEDRU ( )

SILEFUR ( )

SILEMEN ( )

SILENOC ( )

SILEPAR ( )

SILEPRA ( )

SILEURA ( )

SPERARV ( )

SPERDIA ( ) 
FAMILY

LATIN NAME

ENGLISH COMMON NAME

CARYOPHYLLACEAE

CARYOPHYLLACEAE

CARYOPHYLLACEAE

CARYOPHYLLACEAE

CARYOPHYLLACEAE

CARYOPHYLLACEAE

CARYOPHYLLACEAE

CARYOPHYLLACEAE

CARYOPHYLLACEAE

CARYOPHYLLACEAE

CARYOPHYLLACEAE

CARYOPHYLLACEAE

CERATOPHYLLACEAE

CHENOPODIACEAE

CHENOPOD IACEAE

CHENOPODIACEAE

CHENOPODIACEAE

CHENOPODIACEAE

CHENOPOD IACEAE

CHENOPODIACEAE

CHENOPODIACEAE

CHENOPODIACEAE

CHENOPODIACEAE

CHENOPODIACEAE

CHENOPODIACEAE

CHENOPODIACEAE

CHENOPODIACEAE

CHENOPODIACEAE

CHENOPODIACEAE

CHENOPODIACEAE

CHENOPODIACEAE

CHENOPODIACEAE

CHENOPOD IACEAE

CHENOPOD I ACEAE

CHENOPODIACEAE

CHENOPODIACEAE

CHENOPOD IACEAE

CHENOPODIACEAE

CHENOPODIACEAE

CHENOPODIACEAE

CHENOPODIACEAE

CHENOPODIACEAE

CHENOPODIACEAE

CHENOPODIACEAE

CHENOPODIACEAE

CHENOPODIACEAE

CHENOPODIACEAE

CHENOPODIACEAE

CHENOPODIACEAE

COMMELINACEAE

COMPOSITAE

COMPOSITAE

COMPOSITAE

COMPOSITAE
Spergularia marina

Stellaria americana

Stellaria arenicola

Stellaria calycantha

Stellaria crassifolia

Stellaria crispa

Stellaria longifolia

Stellaria longipes

Stellaria media

Stellarla obtusa

Stellaria umbellata

Vaccaria pyramidata

Ceratophy I lum demersum

Atriplex argentea

Atriplex heterosperma

Atriplex hortensis

Atriplex oblongifolia

Atriplex powellii

Atriplex prostrata

Atriplex rosea

Atriplex subspicata

Atriplex suckleyi

Atriplex truncata

Axyris amaranthoides

Bassia hyssopifolia

Chenopodium album

Chenopodium atrovirens

Chenopodium berlandieri

Chenopodium bonus-henricus

Chenopodium capitatum

Chenopodium desiccatum

Chenopodium fremontii

Chenopodium gigantospermum

Chenopodium incanum

Chenopodium leptophy II um

Chenopodium pratericola

Chenopodium rubrum

Chenopodium salinum

Chenopodium subglabrum

Chenopodium watsoni $i$

Corispermum hyssopifolium

Corispermum nitidum

Eurotia lanata

Kochia scoparia

Monolepis nuttalliana

Salicornia europaea

Salsola kali

Suada calceoliformis

Suckleya suckleyana

Tradescantia occidentalis

Achillea millefolium

Achillea ptarmica

Achillea sibirica

Adenocaulon bicolor

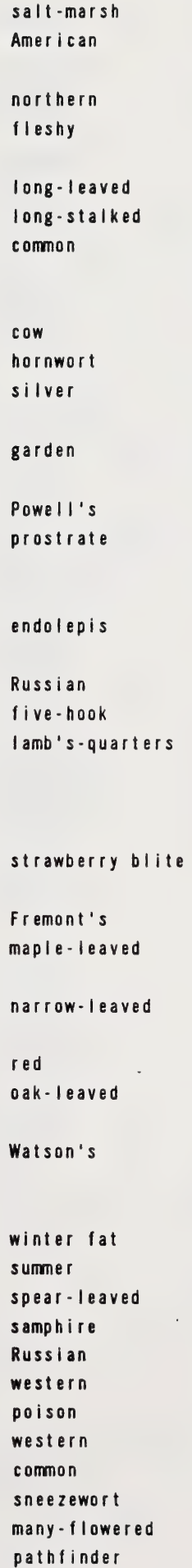

sand spurry

chickweed

chickweed

stitchwort

stitchwort

chickweed

chickweed

chickweed

chickweed

chickweed

chickweed

cockle

saltbush

saltbush

orache

saltbush

saltbush

saltbush

saltbush

saltbush

saltbush

pigweed

bassia

goosefoot

goosefoot

goosefoot

goosefoot

goosefoot

goosefoot

goosefoot

goosefoot

goosefoot

goosefoot

goosefoot

goosefoot

goosefoot

bugseed

bugseed

cypress

goosefoot

thistle

sea-blite

suckleya

spiderwort

yarrow

yarrow

yarrow
CODE CHECK

SPERMAR （）

STELAME ( )

STELARE ( )

STELCAL ( )

STELCRA ( )

STELCRI ( )

STELLON ( )

STELLOG ( )

STELMED ( )

STELOBT ( )

STELUMB ( )

VACCPYR ( )

CERADEM ( )

ATRIARG ( )

ATRIHET ( )

ATRIHOR ( )

ATRIOBL ( )

ATRIPOW ( )

ATRIPRO ( )

ATRIROS ( )

ATRISUB ( )

ATRISUC ( )

ATRITRU ( )

AXYRAMA ()

BASSHYS ( )

CHENALB ( )

CHENATR ( )

CHENBER ( )

CHENBON ( )

CHENCAP ( )

CHENDES (

CHENFRE ( )

CHENGIG ( )

CHENINC ( )

CHENLEP ( )

CHENPRA ( )

CHENRUB ( )

CHENSAL ( )

CHENSUB ( )

CHENWAT ( )

CORIHYS ( )

CORINIT ( )

EUROLAN ( )

KOCHSCO ( )

MONONUT ( )

SALIEUR ( )

SALSKAL ( )

SUAECAL ( )

SUCKSUC ( )

TRADOCC ( )

ACHIMIL ( )

ACHIPTA ( )

ACHISIB ( )

ADENBIC ( ) 
COMPOSITAE COMPOSITAE CONPOSITAE COMPOSITAE COMPOSITAE COMPOSITAE COMPOSITAE COMPOSITAE COMPOSITAE COMPOSITAE COMPOSITAE COMPOSITAE COMPOSITAE COMPOSITAE COMPOSITAE COMPOSITAE COMPOSITAE COMPOSITAE COMPOSITAE COMPOSITAE COMPOSITAE COMPOSITAE COMPOSITAE COMPOSITAE COMPOSITAE COMPOSITAE COMPOSITAE COMPOSITAE COMPOSITAE COMPOSITAE COMPOSITAE COMPOSITAE COMPOSITAE COMPOSITAE COMPOSITAE COMPOSITAE COMPOSITAE COMPOSITAE COMPOSITAE COMPOSITAE COMPOSITAE COMPOSITAE COMPOSITAE COMPOSITAE COMPOSITAE COMPOSITAE COMPOSITAE COMPOSITAE COMPOSITAE COMPOSITAE COMPOSITAE COMPOSITAE COMPOSITAE COMPOSITAE

\begin{tabular}{|c|c|c|}
\hline Agoseris aurantiaca & orange & false dandelion \\
\hline Agoseris glauca & & false dandelion \\
\hline Ambrosia artemisifolia & common & ragweed \\
\hline Ambrosia psilostachya & perennial & ragweed \\
\hline Ambrosia trifida & great & ragweed \\
\hline Anaphalis margaritacea & pearly & everlasting \\
\hline Antennaria alpina & alpine & everlasting \\
\hline Antennaria anaphaloides & tall & everlasting \\
\hline Antennaria aprica & low & everlasting \\
\hline Antennaria corymbosa & corymbose & everlasting \\
\hline Antennaria dimorpha & cushion & everlasting \\
\hline Antennaria lanata & woolly & everlasting \\
\hline Antennaria luzuloides & silvery & everlasting \\
\hline Antennaria monocephala & & everlasting \\
\hline Antennaria neglecta & broad-leaved & everlasting \\
\hline Antennaria parvifolia & small- leaved & everlasting \\
\hline Antennaria pulcherrima & showy & everlasting \\
\hline Antennaria racemosa & racemose & everlasting \\
\hline Antennaria rosea & rosy & everlasting \\
\hline Antennaria umbrinella & brown-bracted mountain & everlasting \\
\hline Anthemis cotula & mayweed & \\
\hline Anthemis tinctoria & yellow & chamomile \\
\hline Arctium Iappa & great & burdock \\
\hline Arctium minus & common & burdock \\
\hline Arctium tomentosum & woolly & burdock \\
\hline Arnica amplexicaulis & & arnica \\
\hline Arnica angustifolia & alpine & arnica \\
\hline Arnica chamissonis & I eafy & arnica \\
\hline Arnica cordifolia & heart-leaved & arnica \\
\hline Arnica diversifolia & |awless & arnica \\
\hline Arnica fulgens & shining & arnica \\
\hline Arnica gracilis & & arnica \\
\hline Arnica latifolia & mountain & arnica \\
\hline Arnica Ionchophylla & spear-leaved & arnica \\
\hline Arnica langifolia & long-leaved & arnica \\
\hline Arnica louiseana & rock & arnica \\
\hline Arnica mollis & cordilleran & arnica \\
\hline Arnica parryi & nodding & arnica \\
\hline Arnica rydbergii & narrow-leaved & arnica \\
\hline Arnica sororia & twin & arnica \\
\hline Artemisia abrotanum & southernwood & \\
\hline Artemisia absinthium & & wormwood \\
\hline Artemisia biennis & biennial & sagewort \\
\hline Artemisia borealis & & wormwood \\
\hline Artemisia campestris & plains & wormwood \\
\hline Artemisia dracunculus & dragonwort & \\
\hline Artemisia frigida & pasture & sagewort \\
\hline Artemisia furcata & & wormwood \\
\hline Artemisia Iongifolia & long-leaved & sage \\
\hline Artemisia ludoviciana & prairie & sagewort \\
\hline Artemisia michauxiana & Michaux's & sage \\
\hline Artemisia norvegica & mountain & sage \\
\hline Artemisia tilesii & Herriot's & sage \\
\hline Aster alpinus & alpine & aster \\
\hline
\end{tabular}

AGOSAUR ()

AGOSGLA ( )

AMBRART ( )

AMBRPSI ( )

AMBRTRI ( )

ANAPMAR ( )

ANTEALP ( )

ANTEANA ( )

ANTEAPR ( )

ANTECOR ( )

ANTEDIM ( )

ANTELAN ( )

ANTELUZ ( )

ANTEMON ( )

ANTENEG ( )

ANTEPAR ( )

ANTEPUL ( )

ANTERAC ( )

ANTEROS ( )

ANTEUMB ( )

ANTHCOT ( )

ANTHTIN ( )

ARCTLAP ( )

ARCTMIN ( )

ARCTTOM ( )

ARNIAMP ( )

ARNIANG ( )

ARNICHA ( )

ARNICOR ( )

ARNIDIV ( )

ARNIFUL ( )

ARNIGRA ( )

ARN ILAT ( )

ARNILOC ( )

ARNILON ( )

ARNILOU ( )

ARNIMOL ( )

ARNIPAR ( )

ARN IRYD ( )

ARNISOR ( )

ARTEABR ( )

ARTEABS ( )

ARTEBIE ( )

ARTEBOR ( )

ARTECAM ( )

ARTEDRA ( )

ARTEFRI ( )

ARTEFUR ( )

ARTELON ( )

ARTELUD ( )

ARTEMIC ( )

ARTENOR ( )

ARTETIL ( )

ASTEALP ( ) 
COMPOSITAE

COMPOSITAE

COMPOSITAE

COMPOSITAE

COMPOSITAE

COMPOSITAE

COMPOSITAE

COMPOSITAE

COMPOSITAE

COMPOSITAE

COMPOSITAE

COMPOSITAE

COMPOSITAE

COMPOSITAE

COMPOSITAE

COMPOSITAE

COMPOSITAE

COMPOSITAE

COMPOSITAE

COMPOSITAE

COMPOSITAE

COMPOSITAE

COMPOSITAE

COMPOSITAE

COMPOSITAE

COMPOSITAE

COMPOSITAE

COMPOSITAE

COMPOSITAE

COMPOSITAE

COMPOSITAE

COMPOSITAE

COMPOSITAE

COMPOSITAE

COMPOSITAE

COMPOSITAE

COMPOSITAE

COMPOSITAE

COMPOSITAE

COMPOSITAE

COMPOSITAE

COMPOSITAE

COMPOSITAE

COMPOSITAE

COMPOSITAE

COMPOSITAE

COMPOSITAE

COMPOSITAE

COMPOSITAE

COMPOSITAE

COMPOSITAE

COMPOSITAE

COMPOSITAE

COMPOSITAE

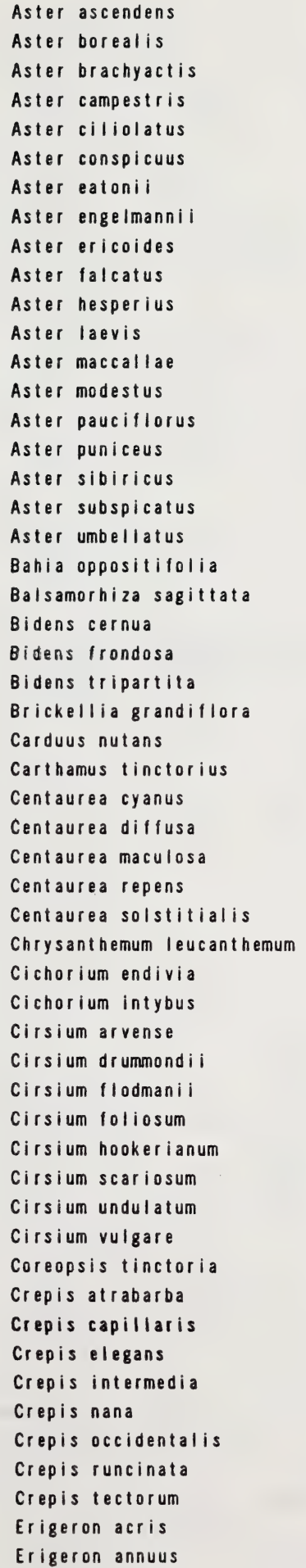

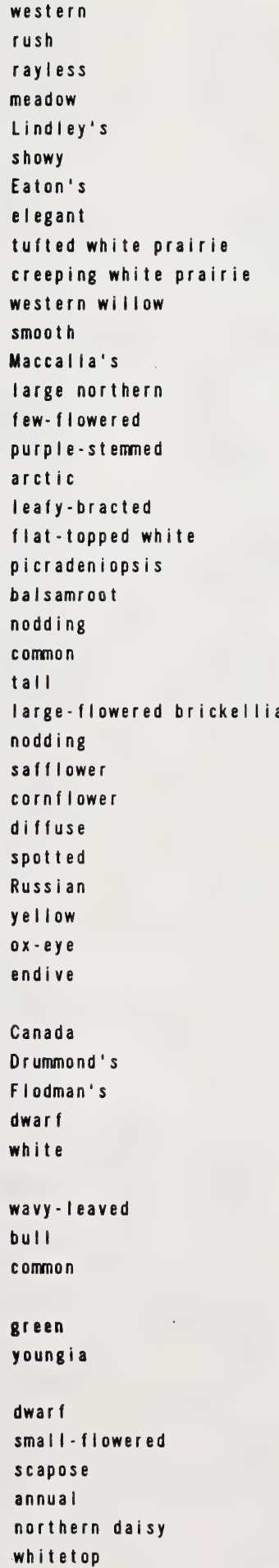

ASTEASC （）

ASTEBOR ( )

ASTEBRA ( )

ASTECAM ( )

ASTECIL ( )

ASTECON ( )

ASTEEAT ( )

ASTEENG ( )

ASTEERI ( )

ASTEFAL ( )

ASTEHES ( )

ASTELAE ( )

ASTEMAC ( )

ASTEMOD ( )

ASTEPAU ( )

ASTEPUN ( )

ASTESIB ( )

ASTESUB ( )

ASTEUMB ( )

BAHIOPP ( )

BALSSAG ( )

BIDECER ( )

BIDEFRO ( )

BIDETRI ( )

BRICGRA ( )

CARDNUT ( )

CARTTIN ( )

CENTCYA ()

CENTDIF ()

CENTMAC ( )

CENTREP ( )

CENTSOL ( )

CHRYLEU ()

CICHEND ( )

CICHINT ( )

CIRSARV ( )

CIRSORU ( )

CIRSFLO ( )

CIRSFOL ( )

CIRSHOO ( )

CIRSSCA ( )

CIRSUND ( )

CIRSVUL ( )

CORETIN ( )

CREPATR ( )

CREPCAP ( )

CREPELE ( )

CREPINT ( )

CREPNAN ( )

CREPOCC ( )

CREPRUN ( )

CREPTEC ( )

ERIGACR ( )

ERIGANN () 
COMPOSITAE COMPOSITAE COMPOSITAE COMPOSITAE COMPOSITAE COMPOSITAE COMPOSITAE COMPOSITAE COMPOSITAE COMPOSITAE COMPOSITAE COMPOSITAE COMPOSITAE COMPOSITAE COMPOSITAE COMPOSITAE COMPOSITAE COMPOSITAE COMPOSITAE COMPOSITAE COMPOSITAE COMPOSITAE COMPOSITAE COMPOSITAE COMPOSITAE COMPOSITAE COMPOSITAE COMPOSITAE COMPOSITAE COMPOSITAE COMPOSITAE COMPOSITAE COMPOSITAE COMPOSITAE COMPOSITAE COMPOSITAE COMPOSITAE COMPOSITAE COMPOSITAE COMPOSITAE COMPOSITAE COMPOSITAE COMPOSITAE COMPOSITAE COMPOSITAE COMPOSITAE COMPOSITAE COMPOSITAE COMPOSITAE COMPOSITAE COMPOSITAE COMPOSITAE COMPOSITAE COMPOSITAE
Erigeron aureus

Erigeron caespitosus

Erigeron canadensis

Erigeron compositus

Erigeron divergens

Erigeron elatus

Erigeron flagellaris

Erigeron glabellus

Erigeron grandiflorus

Erigeron humllis

Erigeron hyssopifolius

Erigeron Ianatus

Erigeron lonchophyllus

Erigeron ochroleucus

Erigeron peregrinus

Erigeron philadelphicus

Erigeron pumilus

Erigeron purpuratus

Erigeron radicatus

Erigeron speciosus

Erigeron trifidus

Erigeron $x$ arthuri $i$

Eupatorium maculatum

Franseria acanthicarpa

Gaillardia aristata

Galinsoga ciliata

Gnaphalium microcephalum

Gnaphalium palustre

Gnaphalium uliginosum

Gnaphalium viscosum

Grindelia squarrosa

Gutierrezia sarothrae

Haplopappus lanceolatus

Haplopappus Iyallii

Haplopappus spinulosus

Haplopappus uniflorus

Helenium autumnale

Helianthus annuus

Helianthus couplandi

Helianthus maximiliani

Helianthus nuttallii

Helianthus subrhomboideus

Heterotheca villosa

Hieracium albiflorum

Hieracium aurantiacum

Hieracium cynoglossoides

Hieracium triste

Hieracium umbellatum

Hymenopappus filifolius

Hymenoxys acaulis

Hymenoxys richardsoni $i$

Iva axillaris

Iva xanthifolia

Lactuca biennis

yellow
tufted
horseweed
compound
tall
creeping
smooth
large-flowered
purple
wild
woolly
hirsute
yellow-bracted alpine
wandering
Philadelphia
hairy
pale
dwarf
showy
cliff
spotted Joe-pye weed
bur
gaillardia
galinsoga
common
marsh
low
clammy

c I ammy

I ance-leaved

sticky

spiny

one-flowered

sneezeweed

common annual

annual

narrow-leaved

common tall

rhombic-leaved

golden

white

orange

woolly

alpine

narrow-leaved

tufted

butte

Colorado

povertyweed

false

tall blue

\section{daisy \\ fleabane \\ fleabane \\ fleabane \\ fleabane \\ fleabane \\ fleabane \\ daisy \\ daisy \\ daisy \\ daisy \\ fleabane \\ daisy \\ daisy \\ fleabane \\ daisy \\ daisy \\ fleabane \\ fleabane \\ fleabane \\ daisy}

ragweed

cudweed

cudweed

cudwe ed

cudweed

gumweed

broomweed

pyrrocoma

stenotus

i ronplant

ironplant

sunflower

sunflower

sunflower

sunflower

sunflower

aster

hawkweed

hawkweed

hawkweed

hawkweed

hawkweed

hymenopappus

marigold

rubber-plant

ragweed

lettuce
ERIGAUR ( )

ERIGCAE ( )

ERIGCAN ( )

ERIGCOM ( )

ERIGDIV ( )

ERIGELA ( )

ERIGFLA ( )

ERIGGLA ( )

ERIGGRA ( )

ERIGHUM ( )

ERIGHYS ( )

ERIGLAN ( )

ERIGLON ( )

ERIGOCH ( )

ERIGPER ( )

ERIGPHI ( )

ERIGPUM ( )

ERIGPUR ( )

ERIGRAD ( )

ERIGSPE ( )

ERIGTRI ( )

ERIGART ( )

EUPAMAC ( )

FRANACA ( )

GAILARI ( )

GALICIL ( )

GNAPMIC ( )

GNAPPAL ( )

GNAPULI ( )

GNAPVIS ( )

GRINSQU ( )

GUTISAR ( )

HAPLLAN ( )

HAPLLYA ()

HAPLSPI ()

HAPLUNI ( )

HELEAUT ( )

HELIANN ( )

HELICOU ( )

HELIMAX ( )

HELINUT ( )

HELISUB ( )

HETEVIL ( )

HIERALB ( )

HIERAUR ( )

HIERCYN ( )

HIERTRI ( )

HIERUMB ( )

HYMEFIL ( )

HYMEACA ( )

HYMERIC ( )

IVAAXIL ( )

IVAXANT ( )

LACTBIE ( ) 
COMPOSITAE COMPOSITAE COMPOSITAE COMPOSITAE COMPOSITAE COMPOSITAE COMPOSITAE COMPOSITAE COMPOSITAE COMPOSITAE COMPOSITAE COMPOSITAE COMPOSITAE COMPOSITAE COMPOSITAE COMPOSITAE COMPOSITAE COMPOSITAE COMPOSITAE COMPOSITAE COMPOSITAE COMPOSITAE COMPOSITAE COMPOSITAE COMPOSITAE COMPOSITAE COMPOSITAE COMPOSITAE COMPOSITAE COMPOSITAE COMPOSITAE COMPOSITAE COMPOSITAE COMPOSITAE COMPOSITAE COMPOSITAE COMPOSITAE COMPOSITAE COMPOSITAE COMPOSITAE COMPOSITAE COMPOSITAE COMPOSITAE COMPOSITAE COMPOSITAE COMPOSITAE COMPOSITAE COMPOSITAE COMPOSITAE COMPOSITAE COMPOSITAE COMPOSITAE COMPOSITAE COMPOSITAE

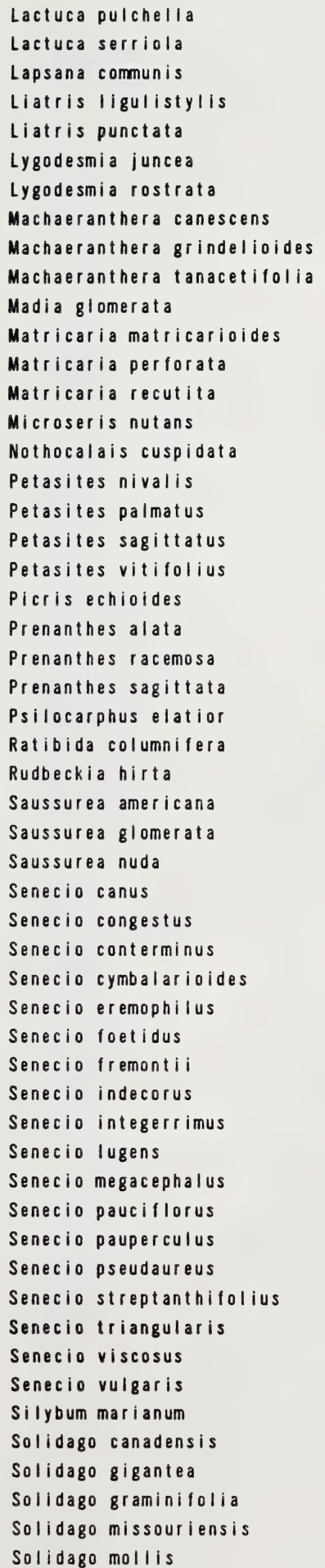

LACTPUL ( )

LACTSER ( )

LAPSCOM ( )

LIATLIG ( )

LIATPUN ( )

LYGOJUN ( )

LYGOROS ( )

MACHCAN ( )

MACHGRI ( )

MACHTAN ( )

MADIGLO ( )

MATRMAT ( )

MATRPER ( )

MATRREC ( )

MICRNUT ( )

NOTHCUS ( )

PETANIV ( )

PETAPAL ( )

PETASAG ( )

PETAVIT ( )

PICRECH ( )

PRENALA ( )

PRENARC ( )

PRENSAG ( )

PSILELA ( )

RATICOL ( )

RUDBHIR ( )

SAUSAME ()

SAUSGLO ( )

SAUSNUD ( )

SENECAN ( )

SENECOG ( )

SENECON ( )

SENECYM ( )

SENEERE ( )

SENEFOE ( )

SENEFRE ( )

SENEIND ( )

SENEINT ( )

SENELUG ( )

SENEMAG ( )

SENEPAU ( )

SENEPAP ( )

SENEPSE ( )

SENESTR （）

SENETRI ( )

SENEVIS ( )

SENEVUL ( )

SILYMAR ( )

SOLICAN ( )

SOLIGIG ( )

SOLIGRA ( )

SOLIMIS ( )

SOLIMOL () 


\section{COMPOSITAE} COMPOSITAE COMPOSITAE COMPOSITAE COMPOSITAE COMPOSITAE COMPOSITAE COMPOSITAE COMPOSITAE COMPOSITAE COMPOSITAE COMPOSITAE COMPOSITAE COMPOSITAE COMPOSITAE COMPOSITAE COMPOSITAE COMPOSITAE COMPOSITAE COMPOSITAE COMPOSITAE COMPOSITAE COMPOSITAE CONVOLVULACEAE CONVOLVULACEAE CONVOLVULACEAE CRASSULACEAE CRASSULACEAE CRASSULACEAE CRASSULACEAE CRUCIFERAE CRUCIFERAE CRUCIFERAE CRUCIFERAE CRUCIFERAE CRUCIFERAE CRUCIFERAE CRUCIFERAE CRUCIFERAE CRUCIFERAE CRUCIFERAE CRUCIFERAE CRUCIFERAE CRUCIFERAE CRUCIFERAE CRUCIFERAE CRUCIFERAE CRUCIFERAE CRUCIFERAE CRUCIFERAE CRUCIFERAE CRUCIFERAE CRUCIFERAE CRUCIFERAE

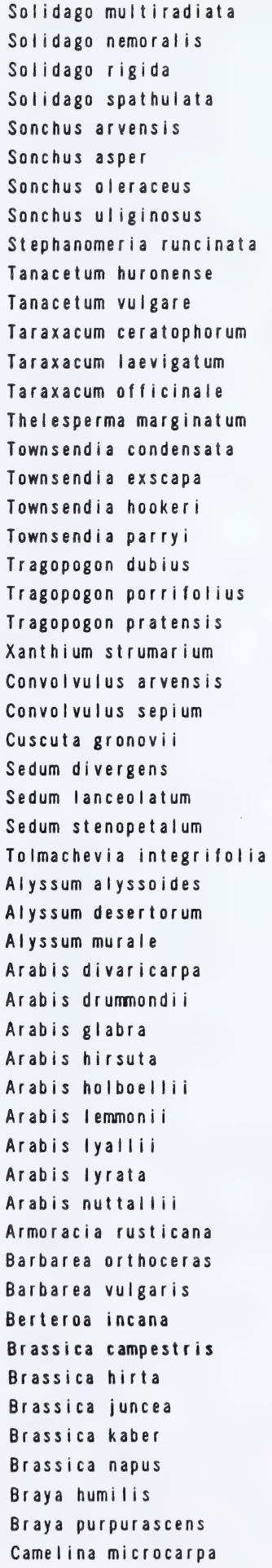

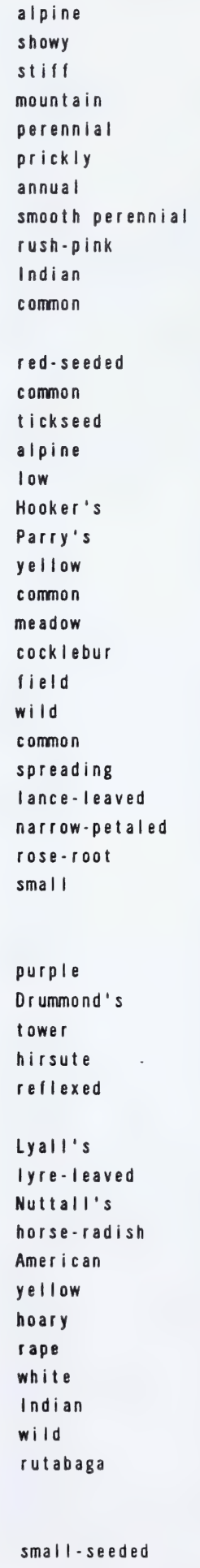

\section{goldenrod goldenrod goldenrod goldenrod sow thistle

false flax
SOLIMUL ( )

SOLINEM ( )

SOLIRIG ( )

SOLISPA ( )

SONCARV ( )

SONCASP ( )

SONCOLE ( )

SONCULI ( )

STEPRUN ( )

TANAHUR ( )

TANAVUL ( )

TARACER ( )

TARALAE ( )

TARAOFF ( )

THELMAR ( )

TOWNCON ( )

TOWNEXS ( )

TOWNHOO ( )

TOWNPAR ( )

TRAGOUB ( )

TRAGPOR ( )

TRAGPRA ( )

XANTSTR ( )

CONVARV ( )

CONVSEP ( )

CUSCGRO ( )

SEDUDIV ( )

SEDULAN ( )

SEDUSTE ( )

TOLMINT ( )

ALYSALY ( )

ALYSDES ( )

ALYSMUR ( )

ARABDIV ( )

ARABDRU ( )

ARABGLA ( )

ARABHIR ( )

ARABHOL ()

ARABLEM ( )

ARABLYA ()

ARABLYR ( )

ARABNUT ( )

ARMORUS ( )

BARBORT ( )

BARBVUL ( )

BERTINC ( )

BRASCAM ( )

BRASHIR ( )

BRASJUN ( )

BRAKSAB ( )

BRASNAP ( )

BRAYHUM ( )

BRAYPUR ( )

CAMEMIC ( ) 
CRUCIFERAE

CRUCIFERAE

CRUCIFERAE

CRUCIFERAE

CRUCIFERAE

CRUCIFERAE

CRUCIFERAE

CRUCIFERAE

CRUCIFERAE

CRUCIFERAE

CRUCIFERAE

CRUCIFERAE

CRUCIFERAE

CRUCIFERAE

CRUCIFERAE

CRUCIFERAE

CRUCIFERAE

CRUCIFERAE

CRUCIFERAE

CRUCIFERAE

CRUCIFERAE

CRUCIFERAE

CRUCIFERAE

CRUCIFERAE

CRUCIFERAE

CRUCIFERAE

CRUCIFERAE

CRUCIFERAE

CRUCIFERAE

CRUCIFERAE

CRUCIFERAE

CRUCIFERAE

CRUCIFERAE

CRUCIFERAE

CRUCIFERAE

CRUCIFERAE

CRUCIFERAE

CRUCIFERAE

CRUCIFERAE

CRUCIFERAE

CRUCIFERAE

CRUCIFERAE

CRUCIFERAE

CRUCIFERAE

CRUCIFERAE

CRUCIFERAE

CRUCIFERAE

CRUCIFERAE

CRUCIFERAE

CRUCIFERAE

CRUCIFERAE

CRUCIFERAE

CRUCIFERAE

CRUCIFERAE
Camelina parodil

Camelina sativa

Capsella bursa-pastoris

Cardamine bellidifolia

Cardamine parviflora

Cardamine pensylvanica

Cardamine pratensis

Cardamine umbellata

Cardaria chalepensis

Cardarla draba

Cardaria pubescens

Conringia orientalis

Descurainia pinnata

Descurainia richardsonii

Descurainia sophia

Diplotaxis muralis

Draba albertina

Draba aurea

Draba borealis

Draba cana

Draba crassifolia

Draba densifolia

Draba lladnizensis

Draba glabella

Draba incerta

Draba kananaskis

Draba lonchocarpa

Draba longipes

Draba macounii

Draba nemorosa

Draba nivalis

Draba oligosperma

Draba paysonii

Draba porsildii

Draba praealta

Draba reptans

Draba stenoloba

Draba ventosa

Draba verna

Eruca sativa

Erucastrum gallicum

Erysimum asperum

Erysimum cheiranthoides

Erysimum inconspicuum

Erysimum pallasii

Halimolobos virgata

Hesperis matronalis

Lepidium bourgeauanum

Lepidium campestre

Lepidium densiflorum

Lepidium latifolium

Lepidium perfoliatum

Lepidium ramosissimum

Lepidium sativum

large-seeded
shepherd's-purse
alpine
small
meadow
mountain

heart-podded

globe-podded

hare's-ear

green tansy

grey tansy

flixweed

sand

golden

thick-leaved

annua I

few-seeded

garden
dog
prairie
wormseed
small-flowered
purple

dame's-rocket

common

common

broad-leaved

perfoliate

branched

garden

\section{false flax \\ false flax \\ bitter cress \\ bitter cress \\ bitter cress \\ bitter cress \\ cress \\ hoary cress \\ hoary cress \\ hoary cress \\ mustard \\ mustard \\ mustard}

rocket

whit low-grass

whit low-grass

whit low-grass

whitlow-grass

whit low-grass

whit low-grass

whitlow-grass

whit low-grass

whitlow-grass

whit low-grass

whitlow-grass

whit low-grass

whitlow-grass

whitlow-grass

whitlow-grass

whitlow-grass

whit low-grass

whit low-grass

whitlow-grass

whit low-grass

whit low-grass

whitlow-grass

whit low-grass

rocket

mustard

rocket

mustard

rocket

rocket

pepper-grass

pepperwort

pepper-grass

pepper-grass

pepper-grass

pepper-grass

cress
CAMEPAR ( )

CAMESAT ( )

CAPSBUR ( )

CARDBEL ( )

CARDPAR ( )

CARDPEN ( )

CARDPRA ( )

CARDUMB ( )

CARDCHA ( )

CARDDRA ( )

CARDPUB ( )

CONRORI ( )

DESCPIN ( )

DESCRIC ( )

DESCSOP ( )

DIPLMUR ( )

DRABALB ( )

DRABAUR ( )

DRABBOR ( )

DRABCAN ()

DRABCRA ( )

DRABDEN ()

DRABFLA ( )

DRABGLA ( )

DRABINC ( )

DRABKAN ()

DRABLON ( )

DRABLOG ( )

DRABMAC ( )

DRABNEM ( )

DRABNIV ( )

DRABOLI ( )

DRABPAY ( )

DRABPOR ( )

DRABPRA ( )

DRABREP ( )

DRABSTE ( )

DRABVEN ( )

DRABVER ( )

ERUCSAT ( )

ERUCGAL ( )

ERYSASP ( )

ERYSCHE ( )

ERYSINC ( )

ERYSPAL ( )

HALIVIR ( )

HESPMAT ( )

LEPIBOU ( )

LEPICAM ( )

LEPIDEN ( )

LEPILAT ( )

LEPIPER ( )

LEPIRAM ( )

LEPISAT ( ) 
CRUCIFERAE

CRUCIFERAE

CRUCIFERAE

CRUCIFERAE

CRUCIFERAE

CRUCIFERAE

CRUCIFERAE

CRUCIFERAE

CRUCIFERAE

CRUCIFERAE

CRUCIFERAE

CRUCIFERAE

CRUCIFERAE

CRUCIFERAE

CRUCIFERAE

CRUCIFERAE

CRUCIFERAE

CRUCIFERAE

CRUCIFERAE

CRUCIFERAE

CRUCIFERAE

CUCURB I TACEAE

DIPSACACEAE

DROSERACEAE

DROSERACEAE

DROSERACEAE

ELATINACEAE

EQUISETACEAE

EQUISETACEAE

EQUISETACEAE

EQUISETACEAE

EQUISETACEAE

EQUISETACEAE

EQUISETACEAE

EQUISETACEAE

EQUISETACEAE

EUPHORBIACEAE

EUPHORB IACEAE

EUPHORBIACEAE

EUPHORB IACEAE

EUPHORB IACEAE

FUMARIACEAE

FUMARIACEAE

GENTIANACEAE

GENT I ANACEAE

GENTIANACEAE

GENTIANACEAE

GENTIANACEAE

GENTIANACEAE

GENTI ANACEAE

GENTIANACEAE

GENT I ANACEAE

GENT I ANACEAE

GENTI ANACEAE

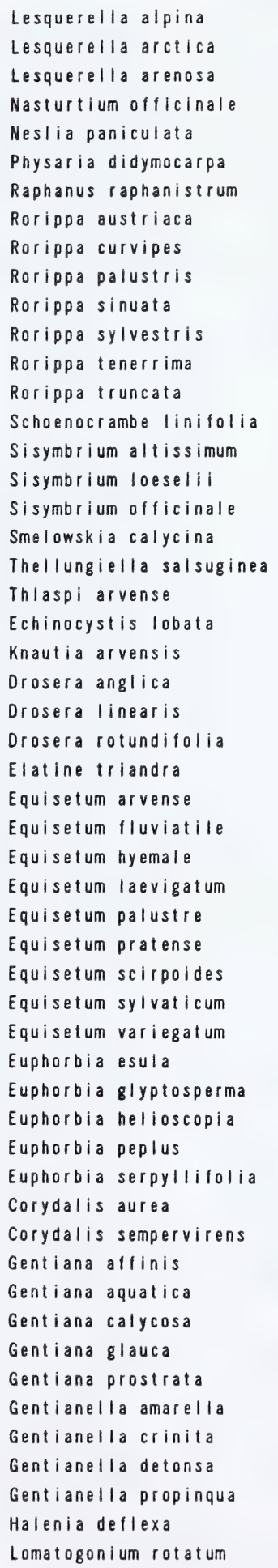

\begin{tabular}{|c|c|}
\hline spatulate & bladderpod \\
\hline northern & bladderpod \\
\hline sand & bladderpod \\
\hline water & cress \\
\hline ball & mustard \\
\hline double & bladderpod \\
\hline wild & radish \\
\hline \multirow[t]{2}{*}{ Austrian } & cress \\
\hline & yellow cress \\
\hline marsh & yellow cress \\
\hline spreading & yellow cress \\
\hline creeping & yellow cress \\
\hline slender & cress \\
\hline blunt-leaved & yellow cress \\
\hline narrow-l eaved & mustard \\
\hline tumbling & mustard \\
\hline tall hedge & mustard \\
\hline hedge & mustard \\
\hline silver & rockcress \\
\hline mouse-ear & cress \\
\hline \multicolumn{2}{|l|}{ st inkweed } \\
\hline wild & cucumber \\
\hline blue & buttons \\
\hline oblong - I eaved & sundew \\
\hline slender-I eaved & sundew \\
\hline round-leaved & sundew \\
\hline \multicolumn{2}{|l|}{ waterwort } \\
\hline common & horsetail \\
\hline swamp & horsetail \\
\hline common & scouring rush \\
\hline smooth & scouring rush \\
\hline marsh & horsetail \\
\hline meadow & horsetail \\
\hline dwarf & scouring rush \\
\hline woodland & horsetail \\
\hline variegated & horsetail \\
\hline leafy & spurge \\
\hline thyme- I eaved & spurge \\
\hline \multicolumn{2}{|l|}{ wartweed } \\
\hline petty & spurge \\
\hline thyme- I eaved & spurge \\
\hline golden & corydalis \\
\hline pink & corydalis \\
\hline prairie & gentian \\
\hline moss & gentian \\
\hline mountain & gentian \\
\hline alpine & gentian \\
\hline moss & gentian \\
\hline northern & gentian \\
\hline fringed & gentian \\
\hline northern fringed & gentian \\
\hline \multicolumn{2}{|l|}{ felwort } \\
\hline spurred & gentian \\
\hline marsh & felwort \\
\hline
\end{tabular}

LESQALP ( )

LESQARC ( )

LESQARE ( )

NASTOFF ( )

NESLPAN ( )

PHYSDID ( )

RAPHRAP ( )

RORIAUS ( )

RORICUR ( )

RORIPAL ( )

RORISIN ( )

RORISYL ( )

RORITEN ( )

RORITRU ( )

SCHOLIN ( )

SISYALT ( )

SISYLOE ( )

SISYOFF ( )

SMELCAL ( )

THELSAL ( )

THLAARV ( )

ECHILOB ( )

KNAUARV ( )

DROSANG ( )

DROSLIN ( )

DROSROT ( )

ELATTRI ( )

EQUIARV ( )

EQUIFLU ( )

EQUIHYE ( )

EQUILAE ( )

EQUIPAL ( )

EQUIPRA ( )

EQUISCI ( )

EQUISYL ( )

EQUIVAR ( )

EUPHESU ( )

EUPHGLY ( )

EUPHHEL ( )

EUPHPEP ( )

EUPHSER ( )

CORYAUR ( )

CORYSEM ( )

GENTAFF ( )

GENTAQU ( )

GENTCAL ( )

GENTGLA ( )

GENTPRO ( )

GENTAMA ( )

GENTCRI ( )

GENTDET ( )

GENTPRP ( )

HALEDEF ( )

LOMAROT ( ) 
GERANIACEAE GERANIACEAE GERANIACEAE GERANIACEAE GERANIACEAE GERANIACEAE HALORAGACEAE HALORAGACEAE HIPPURIDACEAE HIPPUR IDACEAE HYDROCHARI TACEAE HYDROPHYLLACEAE HYDROPHYLLACEAE HYDROPHYLLACEAE HYDROPHYLLACEAE HYDROPHYLLACEAE HYDROPHYLLACEAE HYDROPHYLLACEAE HYDROPHYLLACEAE HYDROPHYLLACEAE HYPERICACEAE HYPERICACEAE

IRIDACEAE

IRIDACEAE

IRIDACEAE

ISOETACEAE

ISOETACEAE

JUNCAG INACEAE

JUNCAG INACEAE

LABIATAE

LAB IATAE

LABIATAE

LABIATAE

LABIATAE

LABIATAE

LABIATAE

LABIATAE

LABIATAE

LABIATAE

LAB IATAE

LABIATAE

LABIATAE

LABIATAE

LAB I ATAE

LABIATAE

LABIATAE

LABIATAE

LAB I ATAE

LAB IATAE

LAB IATAE

LEGUMINOSAE

LEGUMINOSAE

LEGUMINOSAE

LE GUMINOSAE

\begin{tabular}{|c|c|}
\hline $\begin{array}{l}\text { Erodium cicutarium } \\
\text { Geranium bicknellii }\end{array}$ & $\begin{array}{l}\text { stork's-bill } \\
\text { Bicknell's }\end{array}$ \\
\hline Geranium carolinianum & Carolina wild \\
\hline Geranium erianthum & \\
\hline Geranium richardsonii & wild white \\
\hline Geranium viscosissimum & sticky purple \\
\hline Myriophyllum exalbescens & spiked \\
\hline Myriophyllum verticillatum & \\
\hline Hippuris montana & mountain \\
\hline Hippuris vulgaris & \\
\hline Elodea longivaginita & \\
\hline Ellisia nyctelea & waterpod \\
\hline Hydrophy I I um capitatum & woollen-breeches \\
\hline Nemophila breviflora & small \\
\hline Phacelia franklinii & Franklin's \\
\hline Phacelia hastata & silver-leaved \\
\hline Phacelia linearis & I inear - I eaved \\
\hline Phacelia Iyallii & Lyall's \\
\hline Phacelia sericea & silky \\
\hline Romanzoffia sitchensis & Sitka \\
\hline Hypericum formosum & western \\
\hline Hypericum majus & large Canada \\
\hline Iris missouriensis & western blue \\
\hline Sisyrinchium montanum & common \\
\hline Sisyrinchium septentrionale & \\
\hline Isoetes bolanderi & \\
\hline I soetes echinospora & \\
\hline Triglochin maritima & seaside \\
\hline Triglochin palustris & slender \\
\hline Agastache foeniculum & giant \\
\hline Dracocephalum parviflorum & American \\
\hline Dracocephalum thymiflorum & thyme-leaved \\
\hline Galeopsis speciosa & yellow \\
\hline Galeopsis tetrahit & \\
\hline Glecoma hederacea & ground \\
\hline Hedeoma hispidum & pennyroyal \\
\hline Lamium amplexicaule & henbit \\
\hline Leonurus cardiaca & motherwort \\
\hline Lycopus americanus & \\
\hline Lycopus asper & western \\
\hline Lycopus uniflorus & northern \\
\hline Mentha arvensis & wild \\
\hline Mentha spicata & spearmint \\
\hline Monarda fistulosa & wild \\
\hline Nepeta cataria & catnip \\
\hline Physostegia parviflora & false \\
\hline Prunella vulgaris & heal-all \\
\hline Salvia nemorosa & wood \\
\hline Scutellaria galericulata & marsh skullcap \\
\hline Stachys palustris & marsh \\
\hline Astragalus aboriginum & Indian \\
\hline Astragalus alpinus & alpine \\
\hline Astragalus americanus & American \\
\hline Astragalus bisulcatus & two-grooved \\
\hline
\end{tabular}

ERODCIC ( )

GERABIC ( )

GERACAR ( )

GERAERI ( )

GERARIC ( )

GERAVIS ( )

MYRIEXA ( )

MYRIVER ( )

HIPPMON ( )

HIPPVUL ( )

ELODLON ( )

ELLINYC ( )

HYDRCAP ()

NEMOBRE ( )

PHACFRA ( )

PHACHAS ( )

PHACLIN ( )

PHACLYA ( )

PHACSER ( )

ROMASIT ( )

HYPEFOR ( )

HYPEMAS ( )

IRISMIS ( )

SISYMON ( )

SISYSEP ( )

ISOEBOL ( )

ISOEECH ( )

TRIGMAR ( )

TRIGPAL ( )

AGASFOE ()

DRACPAR ( )

DRACTHY ( )

GALESPE ( )

GALETET ( )

GLECHED ( )

HEDEHIS ( )

LAMIAMP ( )

LEONCAR ( )

LYCOAME ( )

LYCOASP ( )

LYCOUNI ( )

MENTARV ( )

MENTSPI ( )

MONAFIS ( )

NEPECAT ( )

PHYSPAR ( )

PRUNVUL ( )

SALVNEM ( )

SCUTGAL ( )

STACPAL ( )

ASTRABO ()

ASTRALP ( )

ASTRAME ( )

ASTRBIS ( ) 
LEGUMINOSAE LEGUMINOSAE LEGUMINOSAE LEGUMINOSAE LEGUMINOSAE LEGUMINOSAE LEGUMINOSAE LEGUMINOSAE LEGUMINOSAE LEGUMINOSAE LEGUMINOSAE LEGUMINOSAE LEGUMINOSAE LEGUMINOSAE LEGUMINOSAE LEGUMINOSAE LEGUMINOSAE LEGUMINOSAE LEGUMINOSAE LEGUMINOSAE LEGUMINOSAE LEGUMINOSAE LEGUMINOSAE LEGUMINOSAE LEGUMINOSAE LEGUMINOSAE LEGUMINOSAE LEGUMINOSAE LEGUMINOSAE LEGUMINOSAE LEGUMINOSAE LEGUMINOSAE LEGUMI NOSAE

LEGUMINOSAE

LEGUMINOSAE

LEGUMINOSAE

LEGUMINOSAE

LEGUMINOSAE

LEGUMINOSAE

LEGUMINOSAE

LEGUMINOSAE

LEGUMINOSAE

LEGUMINOSAE

LEGUMINOSAE

LEGUMINOSAE

LEGUMINOSAE

LEGUMINOSAE

LEGUMINOSAE

LEGUMINOSAE

LEGUMINOSAE

LEGUMINOSAE

LEGUMINOSAE

LEGUMI NOSAE

LEGUMINOSAE
Astragalus bodini

Astragalus bourgovii

Astragalus canadensis

Astragalus cicer

Astragalus crassicarpus

Astragalus dasyglottis

Astragalus drummondi

Astragalus eucosmus

Astragalus flexuosus

Astragalus gilviflorus

Astragalus kentrophyta

Astragalus lotiflorus

Astragalus miser

Astragalus missouriensis

Astragalus pectinatus

Astragalus purshii

Astragalus robbinsii

Astragalus spatulatus

Astragalus striatus

Astragalus tenellus

Astragalus vexilliflexus

Coronilla varia

Glycyrrhiza lepidota

Hedysarum alpinum

Hedysarum boreale

Hedysarum sulphurescens

Lathyrus ochroleucus

Lathyrus venosus

Lotus corniculatus

Lupinus argenteus

Lupinus lepidus

Lupinus nootkatensis

Lupinus polyphyllus

Lupinus pusillus

Lupinus sericeus

Medicago falcata

Medicago lupulina

Medicago sativa

Melilotus alba

Melilotus officinalis

Onobrychis viciifolia

Ononis arvensis

Oxytropis cusickii

oxytropis deflexa

oxytropis jordalii

Oxytropis lagopus

Oxytropis monticola

oxytropis podocarpa

oxytropis sericea

oxytropis splendens

Oxytropis viscida

Petalostemon candidum

Petalostemon purpureum

Psoralea argophylla milk vetch

milk vetch

Canadian

cicer

milk vetch

milk vetch

ground-plum

purple

Drummond's

slender

cushion

prickly

low

timber

Missouri

narrow-leaved

Pursh's

tufted

ascending purple

loose-flowered

few-flowered

field

wild

alpine

northern

yellow

cream-colored

wild

bird's-foot

silvery perennial

alpine

Nootka

large-leafed

annual

silky perennial

yellow

black

alfalfa

white

yellow

sainfoin

alpine

reflexed

hare-footed

late yellow

inflated

early yellow

showy

viscid

white

purple

silverleat
ASTRBOD ( )

ASTRBOU ( )

ASTRCAN ( )

ASTRCIC ( )

ASTRCRA ( )

ASTRDAS ( )

ASTRDRU ( )

ASTREUC ( )

ASTRFLE ( )

ASTRGIL ( )

ASTRKEN ( )

ASTRLOT ( )

ASTRMIS ( )

ASTRMIO ( )

ASTRPEC ( )

ASTRPUR ( )

ASTRROB ( )

ASTRSPA ( )

ASTRSTR ( )

ASTRTEN ( )

ASTRVEX ( )

COROVAR ( )

GLYCLEP ( )

HEDYALP ( )

HEDYBOR ( )

HEDYSUL ( )

LATHOCH ( )

LATHVEN ( )

LOTUCOR ( )

LUPIARG ( )

LUPILEP ( )

LUPINOO ( )

LUPIPOL ( )

LUPIPUS ( )

LUPISER ( )

MEDIFAL ( )

MEDILUP ( )

MEDISAT ( )

MELIALB ( )

MELIOFF ( )

ONOBVIC ( )

ONONARV ( )

OXYTCUS ( )

OXYTDEF ( )

OXYTJOR ( )

OXYTLAG ( )

OXYTMON ( )

OXYTPOD ( )

OXYTSER ( )

OXYTSPL ( )

OXYTVIS ( )

PETACAN ( )

PETAPUR ( )

PSORARG ( ) 
FAMILY

LATIN NAME

ENGLISH COMMON NAME

CODE

CHECK

LEGUMINOSAE

LEGUMINOSAE

LEGUMINOSAE

LEGUMINOSAE

LEGUMINOSAE

LEGUMINOSAE

LEGUMINOSAE

LEGUMINOSAE

LEGUMINOSAE

LE GUMI NOSAE

LEMNACEAE

LEMNACEAE

LEMNACEAE

LEMNACEAE

LEMNACEAE

LENT IBULAR I ACEAE

LENT IBULAR I ACEAE

LENT IBULAR I ACEAE

LENT IBULAR I ACEAE

LENT IBULAR I ACEAE

LENT IBULAR IACEAE

LI LAEACEAE

LILIACEAE

IILIACEAE

LILIACEAE

LILIACEAE

LILIACEAE

LILIACEAE

LILIACEAE

LILIACEAE

LILIACEAE

LILIACEAE

LILIACEAE

LILIACEAE

LILIACEAE

IILIACEAE

LILIACEAE

LILIACEAE

LILIACEAE

LILIACEAE

LILIACEAE

LILIACEAE

LILIACEAE

LILIACEAE

LILIACEAE

LILIACEAE

LILIACEAE

LILIACEAE

LILIACEAE

LILIACEAE

LILIACEAE

LINACEAE

LINACEAE

LINACEAE

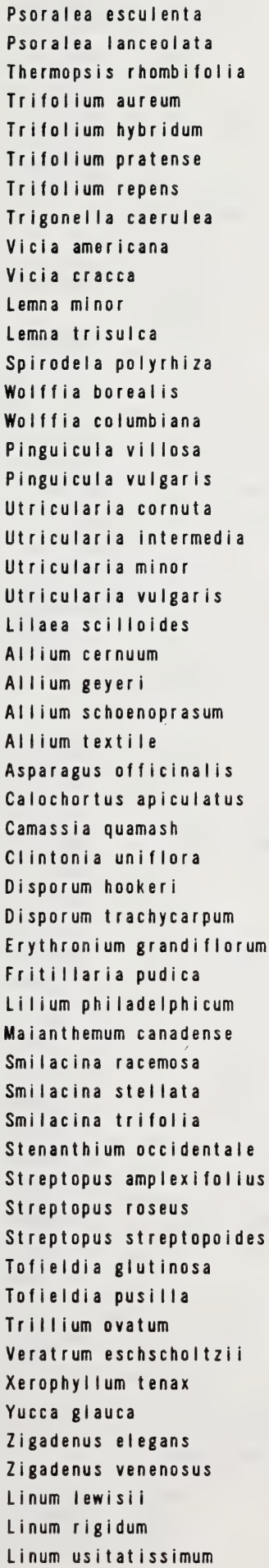

Psoralea esculenta

Indian

scurf

golden

yellow

alsike

red

white

wild

tufted

common

ivy

larger

smal I

common

horned

flat-leaved

small

common

flowering-quill wort

nodding

wild

prairie

asparagus

mariposa

blue

corn

Oregon

glacier

yell owbell

western wood

wild lily-of-the-valley

false

star-flowered

three-leaved

bronzebells

clasping - l eaved

rose mandarin

sticky

bog

western wakerobin

green false

bear-grass

soapweed

white

death

wild blue

yellow

common

breadroot
pea
bean
clover
clover
clover
clover

vetch

vetch

duckweed

duckweed

duckweed

wa termeal

watermeal

but terwort

butterwort

bladderwort

bladderwort

bladderwort

bladderwort

onion

onion

chives

onion

Iily

camas

lily

fairybells

fairybells

Iily

Iily

Solomon's-seal

Solomon's-seal

Solomon's-seal

twisted-stalk

twisted-stalk

asphode I

asphodel

hellebore

camas

camas

flax

flax

flax
PSORESC ( )

PSORLAN ( )

THERRHO ( )

TRIFAUR ( )

TRIFHYB ( )

TRIFPRA ( )

TRIFREP ( )

TRIGCAE ( )

VICIAME ( )

VICICRA ( )

LEMNMIN ( )

LEMNTRI ( )

SPIRPOL ( )

WOLFBOR ( )

WOLFCOL ( )

PINGVIL ( )

PINGVUL ( )

UTRICOR ( )

UTRIINT ( )

UTRIMIN ( )

UTRIVUL ( )

LILASCI ( )

ALLICER ( )

ALLIGEY ( )

ALLISCH ( )

ALLITEX ( )

ASPAOFF ( )

CALOAPI ( )

CAMAQUA ( )

CLINUNI ( )

DISPHOO ( )

DISPTRA ( )

ERYTGRA ( )

FRITPUD ( )

LILIPHI ( )

MAIACAN ( )

SMILRAC ( )

SMILSTE ( )

SMILTRI ( )

STENOCC ( )

STREAMP ( )

STREROS ( )

STRESTR ( )

TOFIGLU ( )

TOFIPUS ( )

TRILOVA ( )

VERAESC ( )

XEROTEN ( )

YUCCGLA ( )

ZIGAELE ( )

ZIGAVEN ( )

LINULEW ( )

LINURIG ( )

LINUUSI ( ) 
LOASACEAE

LOBELIACEAE

LOBELIACEAE

LOBELIACEAE

LOBELIACEAE

LORANTHACEAE

LYCOPODIACEAE

LYCOPODIACEAE

LYCOPODIACEAE

LYCOPODIACEAE

LYCOPODIACEAE

LYCOPODIACEAE

LYCOPODIACEAE

LYCOPODIACEAE

IYTHRACEAE

MALVACEAE

MALVACEAE

MALVACEAE

MALVACEAE

MALVACEAE

MALVACEAE

MARS ILEACEAE

MENYANTHACEAE

MONOTROPACEAE

MONOTROPACEAE

MONOTROPACEAE

NA J ADACEAE

NYCTAGINACEAE

NYCTAGINACEAE

NYCTAGINACEAE

NYMPHAEACEAE

NYMPHAEACEAE

ONAGRACEAE

ONAGRACEAE

ONAGRACEAE

ONAGRACEAE

ONAGRACEAE

ONAGRACEAE

ONAGRACEAE

ONAGRACEAE

ONAGRACEAE

ONAGRACEAE

ONAGRACEAE

ONAGRACEAE

ONAGRACEAE

ONAGRACEAE

ONAGRACEAE

ONAGRACEAE

ONAGRACEAE

ONAGRACEAE

ONAGRACEAE

ONAGRACEAE

ONAGRACEAE

ONAGRACEAE
Mentzelia decapetala

Downingia laeta

Lobelia dortmanna

Lobelia kalmi i

Lobolla splcata

Arceuthobium americanum

Lycopodium alpinum

Lycopodium annotinum

Lycopodium clavatum

Lycopodium complanatum

Lycopodium inundatum

Lycopodium obscurum

Lycopodium selago

Lycopodium sitchense

Lythrum salicaria

Abutilon theophrasti

lliamna rivularis

Malva parviflora

Malva rotundifolia

Malva sylvestris

Malva verticillata

Marsilea vestita

Menyanthes trifoliata

Monotropa hypopitys

Monotropa uniflora

Pterospora andromedea

Najas flexilis

Abronia micrantha

Mirabilis hirsuta

Mirabilis nyctaginea

Nuphar variegatum

Nymphaea tetragona

Boisduvalia glabella

Circaea alpina

Epilobium anagallidifolium

Epilobium angustifolium

Epilobium ciliatum

Epilobium clavatum

Epilobium glaberrimum

Epilobium halleanum

Epilobium hornemannii

Epilobium lactiflorum

Epilobium latifolium

Epilobium leptocarpum

Epilobium leptophyllum

Epilobium luteum

Epilobium mirabile

Epilobium palustre

Epilobium paniculatum

Epilobium saximontanum

Gaura coccinea

Gayophytum racemosum

Oenothera andina

Oenothera biennis sand-lily

downingia

lobelia

lobelia

lobolla

mistletoe

club-moss

club-moss

stiff

running-pine

ground-cedar

club-moss

ground-pine

mountain

ground-fir

purple

velvetleaf

wild

small - fl owered

round-leaved

high

whorled

hairy

pinesap

Indian-pipe

pinedrops

slender

sand

hairy

heart-leaved

yellow

white

smooth

small enchanter's

alpine

northern

naiad

verbena

umbrel l awort

umbrell awort

pond-lily

water-lily

boisduvalia

nightshade

willowherb

fireweed

will owherb

willowherb

willowherb

willowherb

Hornemann's

broad-leaved

marsh

marsh

annual

Rocky Mountain

scarlet butterflyweed

low

upland

yellow
MENTDEC ( )

DOWNLAE ( )

LOBEDOR ( )

LOBEKAL ( )

LOBESPI ( )

ARCEAME ( )

LYCOALP ( )

LYCOANN ( )

LYCOCLA ( )

LYCOCOM ( )

LYCOINU ( )

LYCOOBS ( )

LYCOSEL ( )

LYCOSIT ( )

LYTHSAL ( )

ABUTTHE ( )

ILIARIV ( )

MALVPAR ( )

MALVROT ( )

MALVSYL ( )

MALVVER ( )

MARSVES ( )

MENYTRI ( )

MONOHYP ( )

MONOUNI ( )

PTERAND ( )

NAJAFLE ( )

ABROMIC ( )

MIRAHIR ( )

MIRANYC ()

NUPHVAR ( )

NYMPTET ( )

BOISGLA ( )

CIRCALP ( )

EPILANA ( )

EPILANG ( )

EPILCIL ( )

EPILCLA ( )

EPILGLA ( )

EPILHAL ( )

EPILHOR ( )

EPILLAC ( )

EPILLAT ( )

EPILLEP ( )

EPILLET ( )

EPILLUT ( )

EPILMIR ( )

EPILPAL ( )

EPILPAN ( )

EPILSAX ( )

GAURCOC ( )

GAYORAC ( )

OENOAND ( )

OENOBIE ( ) 
ONAGRACEAE

ONAGRACEAE

ONAGRACEAE

ONAGRACEAE

ONAGRACEAE

OPHIOGLOSSACEAE

OPHIOGLOSSACEAE

OPHIOGLOSSACEAE

OPHIOGLOSSACEAE

OPHIOGLOSSACEAE

OPHIOGLOSSACEAE

OPHIOGLOSSACEAE

OPHIOGLOSSACEAE

ORCHIDACEAE

ORCHIDACEAE

ORCHIDACEAE

ORCHIDACEAE

ORCHIDACEAE

ORCHIDACEAE

ORCHIDACEAE

ORCHIDACEAE

ORCHIDACEAE

ORCHIDACEAE

ORCH IDACEAE

ORCHIDACEAE

ORCHIDACEAE

ORCHIDACEAE

ORCHIDACEAE

ORCHIDACEAE

ORCHIDACEAE

ORCHIDACEAE

ORCHIDACEAE

ORCHIDACEAE

ORCHIDACEAE

ORCHIDACEAE

ORCHIDACEAE

ORCHIDACEAE

ORCHIDACEAE

OROBANCHACEAE

OROBANCHACEAE

OROBANCHACEAE

OROBANCHACEAE

PAPAVERACEAE

PAPAVERACEAE

PAPAVERACEAE

PAPAVERACEAE

PAPAVERACEAE

PARNASSI ACEAE

PARNASSIACEAE

PARNASSIACEAE

PARNAS I ACEAE

PLANTAGINACEAE

PLANTAGINACEAE

PLANTAGINACEAE

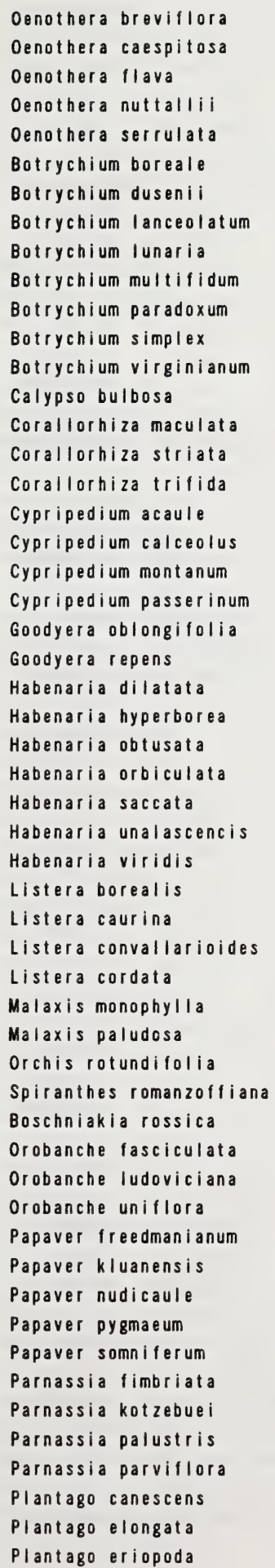

taraxia
butte
yellow
white
shrubby
lance-leaved
moonwort
leather
paradoxical
Virginia
Venus-slipper
spotted
striped
pale
stemless
yellow
mountain
northern
lesser
tall white
northern green
blunt-leaved
large round-leaved bog
slender bog
Alaska bog
bracted
northern
western
broad-lipped
heart-leaved
pol

bog

round-leaved

hooded

clustered

Louisiana

one-flowered

Freedman's

alpine

I celand

alpine

opium

fringed

small

northern

small northern

western

I inear-leaved

saline

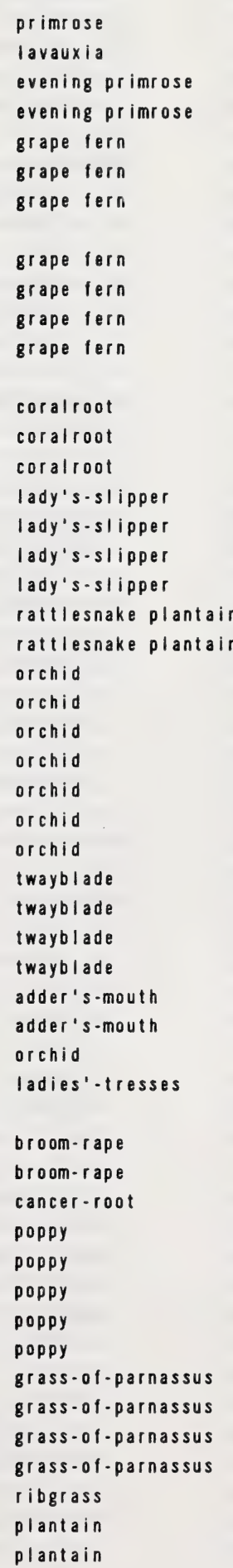

OENOBRE ( )

OENOCAE ( )

OENOFLA ( )

OENONUT ( )

OENOSER ( )

BOTRBOR ( )

BOTRDUS ( )

BOTRLAN ( )

BOTRLUN ( )

BOTRMUL ( )

BOTRPAR ( )

BOTRSIM ( )

BOTRVIR ( )

CALYBUL ( )

CORAMAC ( )

CORASTR ( )

CORATRI ( )

CYPRACA ( )

CYPRCAL ()

CYPRMON ( )

CYPRPAS ( )

GOODOBL ( )

GOODREP ( )

HABEDIL ()

HABEHYP ()

HABEOBT ()

HABEORB ( )

HABESAC ( )

HABEUNA ()

HABEVIR ( )

LISTBOR ( )

LISTCAU ( )

LISTCON ( )

LISTCOR ( )

MALAMON ( )

MALAPAL ( )

ORCHROT ( )

SPIRROM ( )

BOSCROS ( )

OROBFAS ( )

OROBLUD ( )

OROBUNI ( )

PAPAFRE ()

PAPAKLU ()

PAPANUD ( )

PAPAPYG ()

PAPASOM ( )

PARNFIM ( )

PARNKOT ( )

PARNPAL ( )

PARNPAR ( )

PLANCAN ( )

PLANELO ( )

PLANERI ( ) 
PLANTAG INACEAE PLANTAGINACEAE PLANTAGINACEAE POLEMONIACEAE POLEMON I ACEAE POLEMONIACEAE POLEMONIACEAE POLEMONIACEAE POLEMONIACEAE POLEMONIACEAE POLEMONIACEAE POLEMONIACEAE POLYGALACEAE POLYGALACEAE POLYGONACEAE POLYGONACEAE POLYGONACEAE POLYGONACEAE POLYGONACEAE POLYGONACEAE POLYGONACEAE POLYGONACEAE POLYGONACEAE POLYGONACEAE POLYGONACEAE POLYGONACEAE POLYGONACEAE POLYGONACEAE POLYGONACEAE POLYGONACEAE POLYGONACEAE POLYGONACEAE POLYGONACEAE POLYGONACEAE POLYGONACEAE POLYGONACEAE POLYGONACEAE POLYGONACEAE POLYGONACEAE POLYGONACEAE POLYGONACEAE POLYGONACEAE POLYGONACEAE POLYGONACEAE POLYGONACEAE POLYGONACEAE POLYGONACEAE POLYGONACEAE POLYGONACEAE POLYGONACEAE POLYGONACEAE POLYGONACEAE POLYGONACEAE POLYPODIACEAE
Plantago major

Plantago maritima

Plantago patagonica

Collomia linearis

Linanthus septentrionalis

Microsteris gracilis

Navarretia minima

Phlox alyssifolia

Phlox hoodii

Polemonium acutiflorum

Polemonium pulcherrimum

Polemonium viscosum

Polygala paucifolia

Polygala senega

Eriogonum androsaceum

Eriogonum cernum

Eriogonum flavum

Eriogonum ovalifolium

Fagopyrum esculentum

Fagopyrum tartaricum

Koenigia islandica

Oxyria digyna

Polygonum amphibium

Polygonum arenastrum

Polygonum austiniae

Polygonum bistortoides

Polygonum coccineum

Polygonum convolvulus

Polygonum douglasii

Polygonum engelmanni $i$

Polygonum erectum

Polygonum lapathifolium

Polygonum minimum

Polygonum monspeliense

Polygonum persicaria

Polygonum ramosissimum

Polygonum scandens

Polygonum viviparum

Polygonum watsoni $i$

Rumex acetosa

Rumex acetosella

Rumex britannica

Rumex confertus

Rumex crispus

Rumex dentatus

Rumex longifolius

Rumex maritimus

Rumex occidentalis

Rumex paucifolius

Rumex pseudonatronatus

Rumex stenophyllus

Rumex triangulivalvis

Rumex venosus

Adiantum pedatum

common
sea-side
Pursh's
collomia
linanthus
slender
pin-cushion plant
blue
moss
showy
skunkweed
fringed
seneca
cushion
nodding
yellow
silver-plant
common
tartary
mountain
water
common

western
water
wild
Douglas
slender
striate
pale
least

lady's - thumb

bushy

climbing false

alpine

Watson's

green

sheep

water

curled

toothed

long-leaved

golden

western

alpine sheep

field

narrow-leaved field

narrow-leaved

wild

maidenhair

plantain
plantain
plantain

phlox

phlox

phlox

Jacob's-ladder

Jacob's-ladder

milkwort

snakeroot

umbrella-plant

umbrella-plant

umbrella-plant

buckwheat

buckwheat

sorrel

smartweed

knotweed

knotweed

bistort

smartweed

buckwheat

knotweed

knotweed

knotweed

persicaria

knotweed

knotweed

knotweed

buckwheat

bistort

knotweed

sorrel

sorrel

dock

dock

dock

dock

dock

dock

dock

sorrel

dock

dock

dock

begonia

fern
PLANMAJ ( )

PLANMAR ( )

PLANPAT ()

COLLLIN ( )

LINASEP ( )

MICRGRA ( )

NAVAMIN ()

PHLOALY ( )

PHLOHOO ( )

POLEACU ( )

POLEPUL ( )

POLEVIS ( )

POLYPAU ( )

POLYSEG ( )

ERIOAND ( )

ERIOCER ( )

ERIOFLA ( )

ERIOOVA ( )

FAGOESC ( )

FAGOTAR ( )

KOENISL ( )

OXYRDIG ( )

POLYAMP ( )

POLYARE ( )

POLYAUS ( )

POLYBIS ( )

POLYCOC ( )

POLYCON ( )

POLYDOU ( )

POLYENG ( )

POLYERE ( )

POLYLAP ( )

POLYMIN ( )

POLYMOS ( )

POLYPER ( )

POLYRAM ( )

POLYSCA ( )

POLYVIV ( )

POLYWAT ( )

RUMEACE ( )

RUMEACT ( )

RUMEBRI ( )

RUMECON ( )

RUMECRI ( )

RUMEDEN ( )

RUMELON ( )

RUMEMAR ( )

RUMEOCC ( )

RUMEPAU ( )

RUMEPSE ( )

RUMESTE ( )

RUMETRI ( )

RUMEVEN ( )

ADIAPED ( ) 
POLYPODIACEAE POLYPODIACEAE POLYPODIACEAE POLYPODIACEAE POLYPODIACEAE POLYPODIACEAE POLYPODIACEAE POLYPODIACEAE POLYPODIACEAE POLYPODIACEAE POLYPODIACEAE POLYPODIACEAE POLYPODIACEAE POLYPODIACEAE POLYPODIACEAE POLYPODIACEAE POLYPODIACEAE POLYPODIACEAE POLYPODIACEAE POLYPODIACEAE POLYPODIACEAE POLYPODIACEAE POLYPODIACEAE POLYPODIACEAE POLYPODIACEAE POLYPODIACEAE POLYPODIACEAE POLYPODIACEAE PORTULACACEAE PORTULACACEAE PORTULACACEAE PORTULACACEAE PORTULACACEAE PORTULACACEAE POTAMOGE TONACEAE POTAMOGETONACEAE POTAMOGETONACEAE POTAMOGE TONACEAE POTAMOGETONACEAE POTAMOGE TONACEAE POTAMOGE TONACEAE POTAMOGETONACEAE POTAMOGE TONACEAE POTAMOGE TONACEAE POTAMOGE TONACEAE POTAMOGE TONACEAE POTAMOGETONACEAE POTAMOGE TONACEAE POTAMOgE TONACEAE POTAMOGE TONACEAE PRIMULACEAE PRIMULACEAE PRIMULACEAE PRIMULACEAE

\begin{tabular}{|c|c|}
\hline Asplenium viride & green \\
\hline Athyrium distentifolium & alpine \\
\hline Athyrium filix-femina & lady \\
\hline Cheilanthes feei & slender \\
\hline \multicolumn{2}{|l|}{ Chellanthes gracillima } \\
\hline Cryptogramma acrostichoides & parsley \\
\hline Cryptogramma stollorl & fragllo \\
\hline Cystopteris fragilis & fragile \\
\hline Cystopteris montana & mountain bladder \\
\hline Dryopteris assimilis & broad spinulose \\
\hline Dryopteris carthusiana & narrow spinulose \\
\hline Dryopteris cristata & crested \\
\hline Dryopteris filix-mas & male \\
\hline Dryopteris fragrans & fragrant \\
\hline Gymnocarpium dryopteris & oak \\
\hline Gymnocarpium jessoense & Robert's \\
\hline Matteuccia struthiopteris & ostrich \\
\hline Pellaea atropurpurea & purple \\
\hline Pellaea glabella & purple \\
\hline Phegopteris connectilis & beech \\
\hline \multicolumn{2}{|l|}{ Polypodium hesperium } \\
\hline \multicolumn{2}{|l|}{ Polypodium virginianum } \\
\hline Polystichum lonchitis & northern holly \\
\hline Pteridium aquilinum & bracken \\
\hline Woodsia glabella & smooth \\
\hline Woodsia ilvensis & rusty \\
\hline Woodsia oregana & Oregon \\
\hline Woodsia scopulina & mountain \\
\hline Claytonia lanceolata & western \\
\hline $\mathrm{Claytonia}$ megarhiza & alpine \\
\hline Lewisia pygmaea & dwar $f$ \\
\hline Montia linearis & linear-leaved \\
\hline Montia parvifolia & small - leaved \\
\hline Portulaca oleracea & purslane \\
\hline Potamogeton alpinus & alpine \\
\hline Potamogeton crispus & crisp-leaved \\
\hline Potamogeton filiformis & narrow-leaved \\
\hline Potamogeton foliosus & leafy \\
\hline Potamogeton friesii & Fries' \\
\hline Potamogeton gramineus & various-leaved \\
\hline Potamogeton natans & \\
\hline Potamogeton obtusifolius & blunt-leaved \\
\hline Potamogeton pectinatus & sago \\
\hline Potamogeton praelongus & white-stem \\
\hline Potamogeton pusillus & \\
\hline Potamogeton richardsonii & clasping-leaf \\
\hline Potamogeton robbinsii & Robbins' \\
\hline \multicolumn{2}{|l|}{ Potamogeton strictifolius } \\
\hline Potamogeton vaginatus & large-sheath \\
\hline \multicolumn{2}{|l|}{ Potamogeton zosteriformis } \\
\hline Androsace chamaejasme & sweet-flowered \\
\hline Androsace occidentalis & fairy \\
\hline Androsace septentrionalis & fairy \\
\hline
\end{tabular}

\begin{tabular}{|c|c|}
\hline sploonwort & ASPLVIR \\
\hline spleenwort & ATHYDIS \\
\hline fern & ATHYFIL \\
\hline lip fern & CHE IFEE \\
\hline IIp fern & CHEIGRA \\
\hline fern & CRYPACR \\
\hline rock brako & CRYPSTE \\
\hline fern & CYSTFRA \\
\hline fern & CYSTMON \\
\hline shield fern & DRYOASS \\
\hline shield fern & DRYOCAR \\
\hline shield fern & DRYOCRI \\
\hline fern & DRYOF IL \\
\hline shield fern & DRYOFRA \\
\hline fern & GYMNDRY \\
\hline fern & GYMNJES \\
\hline fern & MATTSTR \\
\hline cliff brake & PELLATR \\
\hline cliff brake & PELLGLA \\
\hline fern & PHEGCON \\
\hline rock tripe & POLYHES \\
\hline rock tripe & POLYVIR \\
\hline fern & POLYLON \\
\hline fern & PTERAQU \\
\hline woodsia & WOODGLA \\
\hline woodsia & WOODILV \\
\hline woodsia & WOODORE \\
\hline woodsia & WOODSCO \\
\hline spring beauty & CLAYLAN \\
\hline spring beauty & CLAYMEG \\
\hline bitter-root & LEWIPYG \\
\hline springbeauty & MONTLIN \\
\hline \multirow[t]{2}{*}{ springbeauty } & MONTPAR \\
\hline & PORTOLE \\
\hline pondweed & POTAALP \\
\hline pondweed & POTACRI \\
\hline pondweed & POTAFIL \\
\hline pondweed & POTAFOL \\
\hline pondweed & POTAFRI \\
\hline pondweed & POTAGRA \\
\hline pondweed & POTANAT \\
\hline pondweed & РОТАОВT \\
\hline pondweed & POTAPEC \\
\hline pondweed & POTAPRA \\
\hline pondweed & POTAPUS \\
\hline pondweed & POTARIC \\
\hline pondweed & POTAROB \\
\hline pondweed & POTASTR \\
\hline pondweed & POTAVAG \\
\hline pondweed & POTAZOS \\
\hline androsace & ANDRCHA \\
\hline cande l abra & ANDROCC \\
\hline cande l abra & ANDRSEP \\
\hline chaffweed & CENTMIN \\
\hline
\end{tabular}


PRIMULACEAE PRIMULACEAE PRIMULACEAE PRIMULACEAE PRIMULACEAE PRIMULACEAE PRIMULACEAE PRIMULACEAE PRIMULACEAE PRIMULACEAE PRIMULACEAE PRIMULACEAE PRIMULACEAE PYROLACEAE PYROLACEAE PYROLACEAE PYROLACEAE PYROLACEAE PYROLACEAE PYROLACEAE PYROLACEAE PYROLACEAE RANUNCULACEAE RANUNCULACEAE RANUNCULACEAE RANUNCULACEAE RANUNCULACEAE RANUNCULACEAE RANUNCULACEAE RANUNCULACEAE RANUNCULACEAE RANUNCULACEAE RANUNCULACEAE RANUNCULACEAE RANUNCULACEAE RANUNCULACEAE RANUNCULACEAE RANUNCULACEAE RANUNCULACEAE RANUNCULACEAE RANUNCULACEAE RANUNCULACEAE RANUNCULACEAE RANUNCULACEAE RANUNCULACEAE RANUNCULACEAE RANUNCULACEAE RANUNCULACEAE RANUNCULACEAE RANUNCULACEAE RANUNCULACEAE RANUNCULACEAE RANUNCULACEAE RANUNCULACEAE
Dodecatheon conjugens

Dodecatheon pulchellum Douglasia montana

Glaux maritima

Lysimachia ciliata

Lysimachia lanceolata

Lysimachia thyrsiflora

Primula egaliksensis

Primula incana

Primula mistassinica

Primula stricta

Trientalis borealis

Trientalis europaea

Moneses uniflora

Orthilia secunda

Pyrola asarifolia

Pyrola bracteata

Pyrola chlorantha

Pyrola elliptica

Pyrola grandiflora

Pyrola minor

Pyrola picta

Aconitum delphinifolium

Actaea rubra

Anemone canadensis

Anemone cylindrica

Anemone lithophila

Anemone multifida

Anemone occidentalis

Anemone parviflora

Anemone patens

Anemone quinquefolia

Anemone richardsonii

Anemone riparia

Aquilegia brevistyla

Aquilegia flavescens

Aquilegia formosa

Aquilegia jonesii

Caltha leptosepala

Caltha natans

Caltha palustris

Coptis trifolia

Delphinium bicolor

Delphinium glaucum

Delphinium nuttallianum

Myosurus aristatus

Myosurus minimus

Ranunculus abortivus

Ranunculus acris

Ranunculus aquatilis

Ranunculus cardiophyllus

Ranunculus circinatus

Ranunculus cymbalaria

Ranunculus eschscholtzii

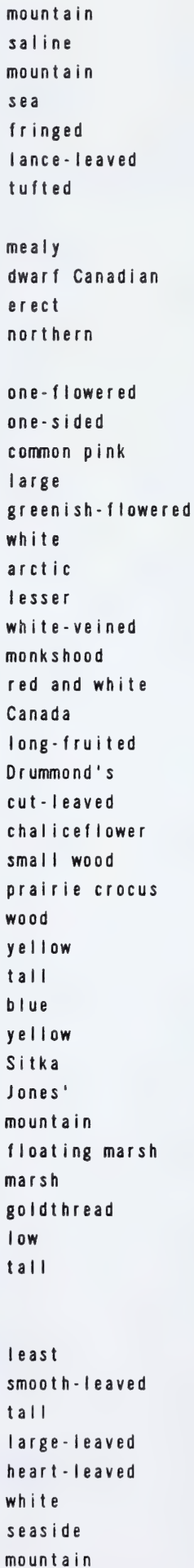

\section{shooting star shooting star dwarf-primula milkwort loosestrife loosestrife loosestrife primrose primrose primrose primrose starflower starflower wintergreen wintergreen wintergreen wintergreen wintergreen wintergreen wintergreen wintergreen wintergreen \\ baneberry anemone anemone anemone anemone}

anemone

anemone

anemone

anemone

columb ine columb ine columb ine columbine marigold marigold marigold

larkspur

larkspur

larkspur

mousetall

mousetail

but tercup

buttercup

watercrowfoot buttercup watercrowfoot buttercup buttercup
DODECON ( )

DODEPUL ( )

DOUGMON ( )

GLAUMAR ( )

LYSICIL ( )

LYSILAN ( )

LYSITHY ( )

PRIMEGA ( )

PRIMINC ( )

PRIMMIS ( )

PRIMSTR ( )

TRIEBOR ( )

TRIEEUR ( )

MONEUNI ( )

ORTHSEC ( )

PYROASA ( )

PYROBRA ( )

PYROCHL ( )

PYROELL ( )

PYROGRA ( )

PYROMIN ( )

PYROPIC ( )

ACONDEL ( )

ACTARUB ( )

ANEMCAN ( )

ANEMCYL ( )

ANEMLIT ( )

ANEMMUL ( )

ANEMOCC ( )

ANEMPAR ( )

ANEMPAT ( )

ANEMQUI ( )

ANEMRIC ( )

ANEMRIP ( )

AQUIBRE ( )

AQUIFLA ( )

AQUIFOR ( )

AQUIJON ( )

CALTLEP ( )

CALTNAT ( )

CALTPAL ( )

COPTTRI ( )

DELPBIC ( )

DELPGLA ( )

DELPNUT ( )

MYOSARI ( )

MYOSMIN ( )

RANUABO ( )

RANUACR ( )

RANUAQU ( )

RANUCAR ( )

RANUCIR ( )

RANUCYM ( )

RANUESC ( ) 
RANUNCULACEAE RANUNCULACEAE RANUNCULACEAE RANUNCULACEAE RANUNCULACEAE RANUNCULACEAE RANUNCULACEAE RANUNCULACEAE RANUNCULACEAE RANUNCULACEAE RANUNCULACEAE RANUNCULACEAE RANUNCULACEAE RANUNCULACEAE RANUNCULACEAE RANUNCULACEAE RANUNCULACEAE RANUNCULACEAE RANUNCULACEAE RANUNCULACEAE RANUNCULACEAE RANUNCULACEAE RANUNCULACEAE ROSACEAE ROSACEAE ROSACEAE ROSACEAE ROSACEAE ROSACEAE ROSACEAE ROSACEAE ROSACEAE ROSACEAE ROSACEAE ROSACEAE ROSACEAE ROSACEAE ROSACEAE ROSACEAE ROSACEAE ROSACEAE ROSACEAE ROSACEAE ROSACEAE ROSACEAE ROSACEAE ROSACEAE ROSACEAE ROSACEAE ROSACEAE ROSACEAE ROSACEAE ROSACEAE ROSACEAE
Ranunculus glaberrimus

Ranunculus gmelinii

Ranunculus grayi

Ranunculus hyperboreus

Ranunculus inamoenus

Ranunculus lapponicus

Ranunculus macouni $i$

Ranunculus nivalis

Ranunculus occidentalis

Ranunculus pedatifidus

Ranunculus pensylvanicus

Ranunculus pygmaeus

Ranunculus repens

Ranunculus reptans

Ranunculus rhomboideus

Ranunculus sceleratus

Ranunculus uncinatus

Ranunculus verecundus

Thalictrum dasycarpum

Thalictrum occidentale

Thalictrum sparsiflorum

Thalictrum venulosum

Trollius albiflorus

Agrimonia striata

Aruncus sylvester

Chamaerhodos erecta

Fragaria vesca

Fragaria virginiana

Geum a leppicum

Geum macrophyl lum

Geum rivale

Geum triflorum

Potentilla anserina

Potentilla argentea

Potentilla arguta

Potentilla bipinnatifida

Potentilla concinna

Potentilla diversifolia

Potentilla drummondi $i$

Potentilla finitima

Potentilla glandulosa

Potentilla gracilis

Potentilla hippiana

Potentilla hookeriana

Potentilla hyparctica

Potentilla multifida

Potentilla multisecta

Potentilla nivea

Potentilla norvegica

Potentilla ovina

Potentilla palustris

Potentilla paradoxa

Potentilla pensylvanica

Potentilla plattensis early

yellow

dwarf

boreal

graceful

Lapland

Macoun's

snow

western

northern

bristly

dwar $f$

creeping

creeping

prairie

celery-leaved

hairy

snow

tall

western

flat-fruited

veiny

globeflower

agrimony

chamaerhodos

woodland

wild

yellow

large-leaved

purple

old man's whiskers

silverweed

silvery

white

plains

early

smooth-leaved

Drummond's

sticky

graceful

woolly

branched

smooth-leaved

snow

rough

marsh

bushy

prairie

low buttercup

watercrowfoot

buttercup

buttercup

buttercup

buttercup

buttercup

buttercup

buttercup

buttercup

buttercup

but tercup

buttercup

spearwort

but tercup

buttercup

but tercup

buttercup

meadow rue

meadow rue

meadow rue

meadow rue

goat's-beard

strawberry

strawberry

avens

avens

avens

cinquefoil

cinquefoil

cinquefoil

cinquefoil

cinquefoil

cinquefoil

cinquefoil

cinquefoil

cinquefoil

cinquefoil

cinquefoil

cinquefoil

cinquefoil

cinquefoil

cinquefoil

cinquefoil

cinquefoil

cinquefoil

cinquefoil

cinquefoil

cinquefoil
RANUGLA ( )

RANUGME ( )

RANUGRA ( )

RANUHYP ( )

RANUINA ( )

RANULAP ( )

RANUMAC ( )

RANUNIV ( )

RANUOCC ( )

RANUPED ( )

RANUPEN ( )

RANUPYG ( )

RANUREP ( )

RANURET ( )

RANURHO ( )

RANUSCE ( )

RANUUNC ( )

RANUVER ( )

THALDAS ( )

THALOCC ( )

THALSPA ( )

THALVEN ( )

TROLALB ( )

AGRISTR ( )

ARUNSYL ( )

CHAMERE ( )

FRAGVES ( )

FRAGVIR ( )

GEUMALE ( )

GEUMMAC ( )

GEUMRIV ( )

GEUMTRI ( )

POTEANS ( )

POTEARG ( )

POTEARU ( )

POTEBIP ( )

POTECON ( )

POTEDIV ( )

POTEDRU ( )

POTEFIN ( )

POTEGLA ( )

POTEGRA ( )

POTEHIP ( )

POTEHOO ( )

POTEHYP ( )

POTEMUL ( )

POTEMUT ( )

POTENIV ( )

POTENOR ( )

POTEOVI ( )

POTEPAL ( )

POTEPAR ( )

POTEPEN ( )

POTEPLA ( ) 
ROSACEAE

ROSACEAE

ROSACEAE

ROSACEAE

ROSACEAE

RUB I ACEAE

RUBIACEAE

RUB IACEAE

RUB I ACEAE

RUB। ACEAE

RUB।ACEAE

RUB IACEAE

RUB I ACEAE

RUBIACEAE

RUPPIACEAE

SANTALACEAE

SANTALACEAE

SARRACEN IACEAE

SAXIFRAGACEAE

SAXIFRAGACEAE

SAXIFRAGACEAE

SAXIFRAGACEAE

SAXIFRAGACEAE

SAXIFRAGACEAE

SAXIFRAGACEAE

SAXIFRAGACEAE

SAXIFRAGACEAE

SAXIFRAGACEAE

SAXIFRAGACEAE

SAXIFRAGACEAE

SAXIFRAGACEAE

SAXIFRAGACEAE

SAXIFRAGACEAE

SAXIFRAGACEAE

SAXIFRAGACEAE

SAXIFRAGACEAE

SAXIFRAGACEAE

SAXIFRAGACEAE

SAXIFRAGACEAE

SAXIFRAGACEAE

SAXIFRAGACEAE

SAXIFRAGACEAE

SAXIFRAGACEAE

SAXIFRAGACEAE

SAXIFRAGACEAE

SAXIFRAGACEAE

SAXIFRAGACEAE

SAXIFRAGACEAE

SAXIFRAGACEAE

SAXIFRAGACEAE

SAXIFRAGACEAE

SAXIFRAGACEAE

SAXIFRAGACEAE

SAXIFRAGACEAE

\begin{tabular}{|c|c|c|}
\hline Potentilla recta & rough-fruited & cinquefoil \\
\hline Potentilla rivalis & brook & cinquefoil \\
\hline Potentilla tridentata & three-toothed & cinquefoil \\
\hline Potentilla uniflora & one-flowered & cinquefoil \\
\hline Potentilla villosa & hairy & cinquefoil \\
\hline Asperula arvensis & quinsywort & \\
\hline Galium aparine & & cleavers \\
\hline Galium boreale & northern & bedstraw \\
\hline Galium labradoricum & Labrador & bedstraw \\
\hline Galium spurium & false & cleavers \\
\hline Galium trifidum & small & bedstraw \\
\hline Galium triflorum & sweet-scented & bedstraw \\
\hline Galium verum & yellow & bedstraw \\
\hline Houstonia longifolia & long-leaved & bluets \\
\hline Ruppia maritima & widgeon-grass & \\
\hline Comandra umbellata & bastard & toadflax \\
\hline Geocaulon lividum & northern comandra & \\
\hline Sarracenia purpurea & pitcher-plant & \\
\hline Chrysosplenium iowense & golden & saxifrage \\
\hline Chrysosplenium tetrandrum & golden & saxifrage \\
\hline \multicolumn{3}{|l|}{ Conimitella williamsii } \\
\hline Heuchera cylindrica & sticky & a l umroot \\
\hline Heuchera glabra & alpine & a l umroot \\
\hline Heuchera parvifolia & smal|- | eaved & a l umr oot \\
\hline Heuchera richardsonii & Richardson's & a l umr oot \\
\hline Leptarrhena pyrolifolia & leather-I eaved & saxifrage \\
\hline Lithophragma glabrum & & rockstar \\
\hline Lithophragma parviflorum & small-flowered & rockstar \\
\hline Mitella breweri & Brewer's & miterwort \\
\hline Mitella nuda & & bishop's-cap \\
\hline Mitella pentandra & & bishop's-cap \\
\hline Mitella trifida & & bishop's-cap \\
\hline Saxifraga adscendens & wedge- leaved & saxifrage \\
\hline Saxifraga aizoides & yellow mountain & saxifrage \\
\hline Saxifraga bronchialis & spotted & saxifrage \\
\hline Saxifraga caespitosa & tufted & saxifrage \\
\hline Saxifraga cernua & nodding & saxifrage \\
\hline Saxifraga ferruginea & . & saxifrage \\
\hline Saxifraga flagellaris & spiderplant & \\
\hline Saxifraga hyperborea & brook & saxifrage \\
\hline Saxifraga lyallii & & saxifrage \\
\hline Saxifraga mertensiana & & saxifrage \\
\hline Saxifraga nelsoniana & & saxifrage \\
\hline Saxifraga nivalis & alpine & saxifrage \\
\hline Saxifraga occidentalis & rhomboid-leaved & saxifrage \\
\hline Saxifraga odontoloma & & saxifrage \\
\hline Saxifraga oppositifolia & purple & saxifrage \\
\hline Saxifraga oregana & & saxifrage \\
\hline Saxifraga tricuspidata & three-toothed & saxifrage \\
\hline Suksdorfia ranunculifolia & & suksdorfia \\
\hline Suksdorfia violacea & blue & suksdorfia \\
\hline \multicolumn{3}{|l|}{ Telesonix heucheriformis } \\
\hline Tellima grandiflora & fringe-cups & \\
\hline Tiarella trifoliata & laceflower & \\
\hline
\end{tabular}

\begin{tabular}{|c|c|}
\hline POTEREC & ( ) \\
\hline POTERIV & ( ) \\
\hline POTETRI & ( ) \\
\hline POTEUNI & ( ) \\
\hline POTEVIL & ( ) \\
\hline ASPEARV & ( ) \\
\hline GALIAPA & ( ) \\
\hline GALIBOR & ( ) \\
\hline GALILAB & ( ) \\
\hline GALISPU & ( ) \\
\hline GALITRF & ( ) \\
\hline GALITRI & ( ) \\
\hline GALIVER & ( ) \\
\hline HOUSLON & ( ) \\
\hline RUPPMAR & ( ) \\
\hline COMAUMB & ( ) \\
\hline GEOCLIV & ( ) \\
\hline SARRPUR & ( ) \\
\hline CHRYIOW & ( ) \\
\hline CHRYTET & ( ) \\
\hline CONIWIL & ( ) \\
\hline HEUCCYL & ( ) \\
\hline HEUCGLA & ( ) \\
\hline HEUCPAR & ( ) \\
\hline HEUCRIC & ( ) \\
\hline LEPTPYR & ( ) \\
\hline LITHGLA & ( ) \\
\hline LITHPAR & ( ) \\
\hline MITEBRE & ( ) \\
\hline MITENUD & ( ) \\
\hline MITEPEN & ( ) \\
\hline MITETRI & ( ) \\
\hline SAXIADS & ( ) \\
\hline SAX $|A| Z$ & ( ) \\
\hline SAXIBRO & ( ) \\
\hline SAXICAE & ( ) \\
\hline SAXICER & ( ) \\
\hline SAXIFER & ( ) \\
\hline SAXIFLA & ( ) \\
\hline SAXIHYP & ( ) \\
\hline SAXILYA & ( ) \\
\hline SAXIMER & ( ) \\
\hline SAXINEL & ( ) \\
\hline SAXINIV & ( ) \\
\hline SAXIOCC & ( ) \\
\hline SAX 1000 & ( ) \\
\hline SAXIOPP & ( ) \\
\hline SAXIORE & ( ) \\
\hline SAXITRI & ( ) \\
\hline SUKSRAN & ( ) \\
\hline SUKSVIO & ( ) \\
\hline TELEHEU & ( ) \\
\hline TELLGRA & ( ) \\
\hline TIARTRI & ( ) \\
\hline
\end{tabular}




\section{SAXIFRAGACEAE}

SCHEUCHZERIACEAE SCROPHULARIACEAE SCROPHULAR IACEAE SCROPHULARIACEAE SCROPHULAR IACEAE SCROPHULARIACEAE SCROPHULARIACEAE SCROPHULAR IACEAE SCROPHULARIACEAE SCROPHULAR I ACEAE SCROPHULAR IACEAE SCROPHULARIACEAE SCROPHULARIACEAE SCROPHULARIACEAE SCROPHULAR IACEAE SCROPHULAR IACEAE SCROPHULARIACEAE SCROPHULAR IACEAE SCROPHULAR IACEAE SCROPHULAR IACEAE SCROPHULARIACEAE SCROPHULAR IACEAE SCROPHULAR IACEAE SCROPHULAR IACEAE SCROPHULAR IACEAE SCROPHULAR IACEAE SCROPHULARIACEAE SCROPHULAR IACEAE SCROPHULAR IACEAE SCROPHULARIACEAE SCROPHULAR IACEAE SCROPHULAR IACEAE SCROPHULARIACEAE SCROPHULAR IACEAE SCROPHULAR IACEAE SCROPHULAR IACEAE SCROPHULARIACEAE SCROPHULAR IACEAE SCROPHULAR IACEAE SCROPHULAR IACEAE SCROPHULAR IACEAE SCROPHULAR IACEAE SCROPHULAR IACEAE SCROPHULARIACEAE SCROPHULAR IACEAE SCROPHULARIACEAE SCROPHULAR IACEAE SCROPHULARIACEAE SCROPHULARIACEAE SCROPHULARIACEAE SCROPHULARIACEAE SCROPHULARIACEAE SCROPHULARIACEAE
Tiarella unifoliata

Scheuchzeria palustris

Besseya wyomingensis

Castilleja cusickii

Castilleja hispida

Castilleja lutescens

Castilleja miniata

Castilleja occidentalis

Castilleja raupii

Castilleja rhexifolia

Castilleja sessiliflora

Chaenorrhinum minus

Collinsia parviflora

Euphrasia arctica

Gratiola neglecta

Limosella aquatica

Linaria dalmatica

Linaria vulgaris

Melampyrum lineare

Mimulus floribundus

Mimulus guttatus

Mimulus lewisii

Odontites serotina

Orthocarpus luteus

Pedicularis arctica

Pedicularis bracteosa

Pedicularis capitata

Pedicularis contorta

Pedicularis flammea

Pedicularis groenlandica

Pedicularis labradorica

Pedicularis lanata

Pedicularis parviflora

Pedicularis racemosa

Pedicularis sudetica

Penstemon albertinus

Penstemon albidus

Penstemon confertus

Penstemon ellipticus

Penstemon eriantherus

Penstemon fruticosus

Penstemon gracilis

Penstemon lyallii

Penstemon nitidus

Penstemon procerus

Rhinanthus minor

Scrophularia lanceolata

Verbascum nigrum

Verbascum phlomoides

Verbascum thapsus

Veronica agrestis

Veronica alpina

Veronica americana

Veronica catenata

sugarscoop
kittentails
yellow
hispid
stiff yellow
common red
lance-leaved
purple
alpine red
downy
dwarf
blue-eyed Mary
eyebright
clammy
mudwort
broad-leaved
butter-and-eggs
cow-wheat

yellow

red

late-flowering

owl-clover

arctic

western

large-flowered

coiled-beak

flame-colored

elephant's-head

Labrador

woolly

swamp

leafy

purple

blue

white

yel low

creeping

crested

shrubby

lilac-flowered

large-flowered

smooth blue

slender blue

yellow rattle

hare

black

woolly

common

prostrate

alpine

American

water

paintbrush
paintbrush
paintbrush
paintbrush
paint-brush
paintbrush
paintbrush
paintbrush
snapdragon
hedge-hyssop
toad-flax

monkey flower

monkey flower

monkey flower

eyebright

lousewort

lousewort

lousewort

lousewort

lousewort

lousewort

lousewort

lousewort

lousewort

rattle

beardtongue

beardtongue

beardtongue

beardtongue

beard tongue

beard tongue

beardtongue

beardiongue

beardtongue

beardtongue

figwort

mullein

mullein

mullein

speedwell

speedwell

brooklime

speedwell
TIARUNI ( )

SCHEPAL ( )

BESSWYO ( )

CASTCUS ( )

CASTHIS ( )

CASTLUT ( )

CASTMIN ( )

CASTOCC ( )

CASTRAU ( )

CAstrhe ( )

CASTSES ( )

CHAEMIN ( )

COLLPAR ( )

EUPHARC ( )

GRATNEG ( )

LIMOAQU ( )

LINADAL ( )

LINAVUL ( )

MELALIN ( )

MIMUFLO ( )

MIMUGUT ( )

MIMULEW ( )

ODONSER ( )

ORTHLUT ( )

PEDIARC ( )

PEDIBRA ( )

PEDICAP ( )

PEDICON ( )

PEDIFLA ( )

PEDIGRO ( )

PEDILAB ( )

PEDILAN ( )

PEDIPAR ( )

PEDIRAC ( )

PEDISUD ( )

PENSALB ( )

PENSALI ( )

PENSCON ( )

PENSELL ( )

PENSERI ( )

PENSFRU ( )

PENSGRA ( )

PENSLYA ( )

PENSNIT ( )

PENSPRO ( )

RHINMIN ( )

SCROLAN ()

VERBNIG ( )

VERBPHL ( )

VERBTHA ( )

VEROAGR ( )

VEROALP ( )

VEROAME ( )

VEROCAT ( ) 
SCROPHULARIACEAE SCROPHULAR IACEAE SCROPHULARIACEAE SCROPHULARIACEAE SCROPHULAR IACEAE SCROPHULAR IACEAE SELAGINELLACEAE SELAGINELLACEAE SELAGINELLACEAE SELAGINELLACEAE SOLANACEAE SOLANACEAE SOLANACEAE SOLANACEAE SOLANACEAE SOLANACEAE SOLANACEAE SPARGAN IACEAE SPARGANIACEAE SPARGANIACEAE SPARGAN IACEAE SPARGAN IACEAE SPARGAN IACEAE TYPHACEAE UMBELLIFERAE UMBE LLIFERAE UMBELLIFERAE UMBELLIFERAE UMBELLIFERAE UMBE LL IFERAE UMBE LLIFERAE UMBE LL IFERAE UMBELLIFERAE UMBELLIFERAE UMBELLIFERAE UMBELLIFERAE UMBELLIFERAE UMBELLIFERAE UMBELLIFERAE UMBELLIFERAE UMBELLIFERAE UMBELLIFERAE UMBE LLIFERAE UMBELLIFERAE UMBELLIFERAE UMBELLIFERAE UMBELLIFERAE UMBELLIFERAE UMBELL IFERAE UMBELLIFERAE UMBE LL IFERAE UMBELLIFERAE UMBELL IFERAE UMBELLIFERAE

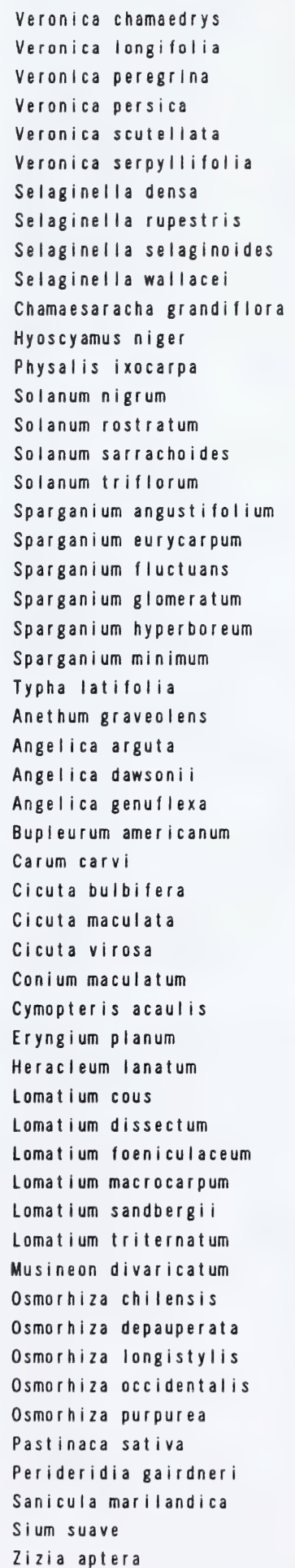

germander
spiked
halry
bird's-eye
marsh
thyme-leaved
prairie
little
little
little
large white ground-cherry
black
tomatillo
black
buffalobur
hairy
wild
narrow-leaved
giant

poison
plains
cross-thistle

cow-parsnip

biscuit-root

mountain wild

hairy-fruited

long-fruited

Sandberg's wild

western wild

leafy

blunt-fruited

spreading

smooth

western

blunt-fruited

wild

squawroot

snakeroot

water

heart-leaved speedwell

speedwell

speedwell

speedwell

speedwell

selaginella

club-moss

club-moss

club-moss

henbane

night shade

nightshade

tomato

bur-reed

bur-reed

bur-reed

bur-reed

bur-reed

bur-reed

cattail

angelica

angelica

angelica

water-heml ock

water-hemlock

water-hemlock

hemlock

cymopterus

cow-parsnip

parsnip

parsley

parsley

parsley

parsley

musineon

sweet cicely

sweet cicely

sweet cicely

sweet cicely

sweet cicely

parsnip

parsnip

Alexanders
VEROCHA ()

VEROLON ( )

VEROPER ( )

VEROPES ( )

VEROSCU ( )

VEROSER ( )

SELADEN ( )

SELARUP ( )

SELASEL ( )

SELAWAL ( )

CHAMGRA ( )

HYOSNIG ( )

PHYSIXO ()

SOLANIG ( )

SOLAROS ( )

SOLASAR ( )

SOLATRI ( )

SPARANG ( )

SPAREUR ( )

SPARFLU ( )

SPARGLO ( )

SPARHYP ()

SPARMIN ( )

TYPHLAT ( )

ANETGRA ( )

ANGEARG ( )

ANGEDAW ( )

ANGEGEN ( )

BUPLAME ( )

CARUCAR ( )

CICUBUL ( )

CICUMAC ( )

CICUVIR ( )

CONIMAC ( )

CYMOACA ( )

ERYNPLA ( )

HERALAN ( )

LOMACOU ( )

LOMADIS ( )

LOMAFOE ( )

LOMAMAC ( )

LOMASAN ( )

LOMATRI ( )

MUSIDIV ( )

OSMOCHI ( )

OSMODEP ( )

OSMOLON ( )

OSMOOCC ( )

OSMOPUR ( )

PASTSAT ( )

PERIGAI ( )

SANIMAR ( )

SIUMSUA ( )

ZIZIAPT ( ) 
FAMILY

$\begin{array}{ll}\text { URTICACEAE } & \text { Parietaria pensylvanica } \\ \text { URTICACEAE } & \text { Urtica dioica } \\ \text { URTICACEAE } & \text { Urtica urens } \\ \text { VALERIANACEAE } & \text { Valeriana dioica } \\ \text { VALERIANACEAE } & \text { Valeriana sitchensis } \\ \text { VERBENACEAE } & \text { Verbena bracteata } \\ \text { VIOLACEAE } & \text { Viola adunca } \\ \text { VIOLACEAE } & \text { Viola arvensis } \\ \text { VIOLACEAE } & \text { Viola canadensis } \\ \text { VIOLACEAE } & \text { Viola glabella } \\ \text { VIOLACEAE } & \text { Viola macloskeyi } \\ \text { VIOLACEAE } & \text { Viola nephrophylla } \\ \text { VIOLACEAE } & \text { Viola nuttallii } \\ \text { VIOLACEAE } & \text { viola orbiculata } \\ \text { VIOLACEAE } & \text { viola palustris } \\ \text { VIOLACEAE } & \text { Viola pedatifida } \\ \text { VIOLACEAE } & \text { Viola renifolia } \\ \text { VIOLACEAE } & \text { Viola selkirkii } \\ \text { VIOLACEAE } & \text { viola tricolor } \\ \text { ZANNICHELLIACEAE } & \text { Zannichellia palustris }\end{array}$

American
common
small
northern
mountain
carpet
early blue
European field
western Canada
yellow wood
Macloskey's
bog
yellow prairie
evergreen
marsh
crowfoot
kidney-leaved
great-spurred
pansy
horned

pellitory

PARIPEN ( )

URTIDIO ( )

URTIURE ( )

VALEDIO ( )

VALESIT ( )

VERBBRA ( )

VIOLADU ( )

VIOLARV ( )

VIOLCAN ( )

VIOLGLA ( )

VIOLMAC ( )

VIOLNEP ( )

VIOLNUT ( )

VIOLORB ( )

VIOLPAL ( )

VIOLPED ( )

VIOLREN ( )

VIOLSEL ( )

VIOLTRI ( )

ZANNPAL ( ) 


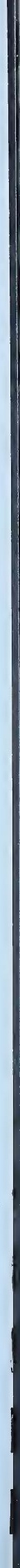


CYPERACEAE

CYPERACEAE

CYPERACEAE

CYPERACEAE

CYPERACEAE

CYPERACEAE

CYPERACEAE

CYPERACEAE

CYPERACEAE

CYPERACEAE

CYPERACEAE

CYPERACEAE

CYPERACEAE

CYPERACEAE

CYPERACEAE

CYPERACEAE

CYPERACEAE

CYPERACEAE

CYPERACEAE

CYPERACEAE

CYPERACEAE

CYPERACEAE

CYPERACEAE

CYPERACEAE

CYPERACEAE

CYPERACEAE

CYPERACEAE

CYPERACEAE

CYPERACEAE

CYPERACEAE

CYPERACEAE

CYPERACEAE

CYPERACEAE

CYPERACEAE

CYPERACEAE

CYPERACEAE

CYPERACEAE

CYPERACEAE

CYPERACEAE

CYPERACEAE

CYPERACEAE

CYPERACEAE

CYPERACEAE

CYPERACEAE

CYPERACEAE

CYPERACEAE

CYPERACEAE

CYPERACEAE

CYPERACEAE

CYPERACEAE

CYPERACEAE

CYPERACEAE

CYPERACEAE

CYPERACEAE
Carex adusta

Carex aenea

Carex albo-nigra

Carex aperta

Carex aquatilis

Carex arcta

Carex atherodes

Carex athrostachya

Carex atrosquama

Carex aurea

Carex backii

Carex bebbii

Carex bipartita

Carex brevior

Carex brunnescens

Carex buxbaumi i

Carex capillaris

Carex capitata

Carex chordorrhiza

Carex concinna

Carex concinnoides

Carex crawe $\mathrm{i}$

Carex crawfordii

Carex curta

Carex deflexa

Carex deweyana

Carex diandra

Carex disperma

Carex douglasii

Carex eburnea

Carex enanderi

Carex epapillosa

Carex filifolia

Carex flava

Carex franklinii

Carex geyeri

Carex glacialis

Carex gynocrates

Carex haydeniana

Carex heleonastes

Carex hoodii

Carex hookerana

Carex houghtoniana

Carex hystricina

Carex illota

Carex interior

Carex kelloggi

Carex lacustris

Carex lanuginosa

Carex lasiocarpa

Carex Ienticularis

Carex leptalea

Carex limosa

Carex livida

browned
silvery-flowered
open
water
narrow
awned
long-bracted
dark-scaled
golden
Back's
Bebb's

brownish

brown

hair-like

capitate

prostrate

beautiful

low northern

Crawe's

Crawford's

short

bent

Dewey's

two-stamened

two-seeded

Douglas

bristle-leaved

thread-leaved

yellow

Franklin's

Geyer's

glacier

northern bog

Hayden's

Hudson Bay

Hood's

Hooker's

sand

porcupine

Kellogg's

lakeshore

woolly

hairy-fruited

lens-fruited

bristle-stalked

mud

livid sedge

sedge

sedge

sedge

sedge

sedge

sedge

sedge

sedge

sedge

sedge

sedge

sedge

sedge

sedge

sedge

sedge

sedge

sedge

sedge

sedge

sedge

sedge

sedge

sedge

sedge

sedge

sedge

sedge

sedge

sedge

sedge

sedge

sedge

sedge

sedge

sedge

sedge

sedge

sedge

sedge

sedge

sedge

sedge

sedge

sedge

sedge

sedge

sedge

sedge

sedge

sedge

sedge

sedge
CAREADU ( )

CAREAEN ( )

CAREALB ( )

CAREAPE ( )

CAREAQU ( )

CAREARC ( )

CAREATH ( )

CAREATR ( )

CAREATS ( )

CAREAUR ( )

CAREBAC ( )

CAREBEB ( )

CAREBIP ( )

CAREBRE ( )

CAREBRU ( )

CAREBUX ( )

CARECAP ( )

CARECAI ( )

CARECHO ()

CARECON ( )

CARECOC ( )

CARECRA ( )

CARECRW ( )

CARECUR ( )

CAREDEF ( )

CAREDEW ( )

CAREDIA ( )

CAREDIS ( )

CAREDOU ( )

CAREEBU ()

CAREENA ( )

CAREEPA ( )

CAREFIL ( )

CAREFLA ( )

CAREFRA ( )

CAREGEY ( )

CAREGLA ( )

CAREGYN ( )

CAREHAY ( )

CAREHEL ( )

CAREHOD ( )

CAREHOO ( )

CAREHOU ( )

CAREHYS ( )

CAREILL ( )

CAREINT ( )

CAREKEL ( )

CARELAC ( )

CARELAN ( )

CARELAS ( )

CARELEN ( )

CARELEP ( )

CARELIM ()

CARELIV ( ) 
CYPERACEAE

CYPERACEAE

CYPERACEAE

CYPERACEAE

CYPERACEAE

CYPERACEAE

CYPERACEAE

CYPERACEAE

CYPERACEAE

CYPERACEAE

CYPERACEAE

CYPERACEAE

CYPERACEAE

CYPERACEAE

CYPERACEAE

CYPERACEAE

CYPERACEAE

CYPERACEAE

CYPERACEAE

CYPERACEAE

CYPERACEAE

CYPERACEAE

CYPERACEAE

CYPERACEAE

CYPERACEAE

CYPERACEAE

CYPERACEAE

CYPERACEAE

CYPERACEAE

CYPERACEAE

CYPERACEAE

CYPERACEAE

CYPERACEAE

CYPERACEAE

CYPERACEAE

CYPERACEAE

CYPERACEAE

CYPERACEAE

CYPERACEAE

CYPERACEAE

CYPERACEAE

CYPERACEAE

CYPERACEAE

CYPERACEAE

CYPERACEAE

CYPERACEAE

CYPERACEAE

CYPERACEAE

CYPERACEAE

CYPERACEAE

CYPERACEAE

CYPERACEAE

CYPERACEAE

CYPERACEAE

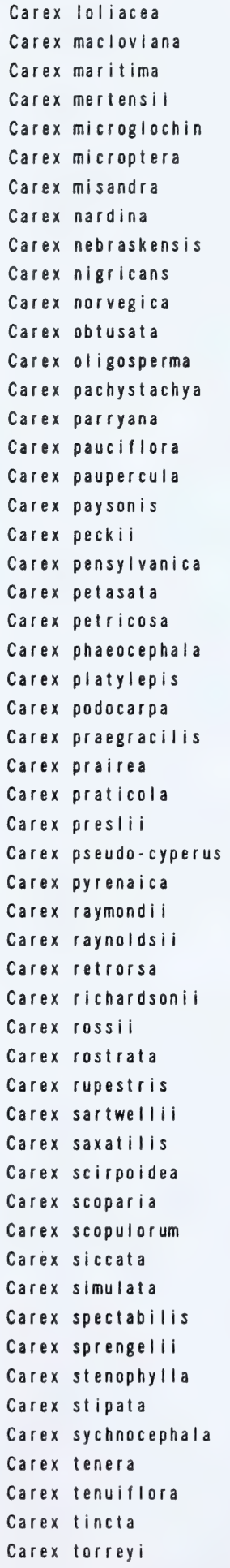

\begin{tabular}{|c|c|}
\hline rye-grass & sedge \\
\hline thick-spike & $\operatorname{sedge}$ \\
\hline seaside & sedge \\
\hline purple & sedge \\
\hline \multirow[t]{2}{*}{ short-awned } & $\operatorname{sedge}$ \\
\hline & $\operatorname{sedg} e$ \\
\hline nodding & sedge \\
\hline fragrant & sedge \\
\hline Nebraska & sedge \\
\hline blackening & sedge \\
\hline Norway & sedge \\
\hline blunt & sedge \\
\hline \multirow[t]{2}{*}{ few-fruited } & sedge \\
\hline & sedge \\
\hline Parry's & sedge \\
\hline few-flowered & $\operatorname{sedge}$ \\
\hline \multirow[t]{2}{*}{ bog } & sedge \\
\hline & sedge \\
\hline Peck's & sedge \\
\hline sun-loving & sedge \\
\hline pasture & sedge \\
\hline stone & sedge \\
\hline \multirow[t]{2}{*}{ head-like } & sedge \\
\hline & sedge \\
\hline alpine & sedge \\
\hline graceful & sedge \\
\hline \multirow[t]{2}{*}{ prairie } & sedge \\
\hline & sedge \\
\hline Presl & sedge \\
\hline cyperus-like & sedge \\
\hline spiked & sedge \\
\hline Raymond's & sedge \\
\hline Raynold's & sedge \\
\hline turned & sedge \\
\hline Richardson's & sedge \\
\hline Ross' & sedge \\
\hline beaked & sedge \\
\hline rock & sedge \\
\hline Sartwell's & sedge \\
\hline rocky-ground & sedge \\
\hline rush-like & $\operatorname{sedge}$ \\
\hline \multirow[t]{2}{*}{ broom } & $\operatorname{sedge}$ \\
\hline & sedge \\
\hline \multirow[t]{3}{*}{ hay } & $\operatorname{sedge}$ \\
\hline & sedge \\
\hline & sedge \\
\hline Sprengel's & $\operatorname{sedge}$ \\
\hline low & sedge \\
\hline awl-fruited & $\operatorname{sedge}$ \\
\hline long-beaked & sedge \\
\hline broad-fruited & sedge \\
\hline thin-flowered & sedge \\
\hline tinged & sedge \\
\hline Torrey's & sedge \\
\hline
\end{tabular}

CARELOL ( ) CAREMAC ( ) 
FAMILY LATIN NAME

CYPERACEAE CYPERACEAE CYPERACEAE CYPERACEAE CYPERACEAE CYPERACEAE CYPERACEAE CYPERACEAE CYPERACEAE CYPERACEAE CYPERACEAE CYPERACEAE CYPERACEAE CYPERACEAE CYPERACEAE CYPERACEAE CYPERACEAE CYPERACEAE CYPERACEAE CYPERACEAE CYPERACEAE CYPERACEAE CYPERACEAE CYPERACEAE CYPERACEAE CYPERACEAE CYPERACEAE CYPERACEAE CYPERACEAE CYPERACEAE CYPERACEAE CYPERACEAE CYPERACEAE CYPERACEAE CYPERACEAE CYPERACEAE CYPERACEAE CYPERACEAE CYPERACEAE GRAMINEAE GRAMINEAE GRAMINEAE GRAMINEAE GRAM INEAE GRAM INEAE GRAMINEAE GRAM INEAE GRAM INEAE GRAMINEAE GRAMINEAE GRAM INEAE GRAMINEAE GRAM INEAE GRAMINEAE

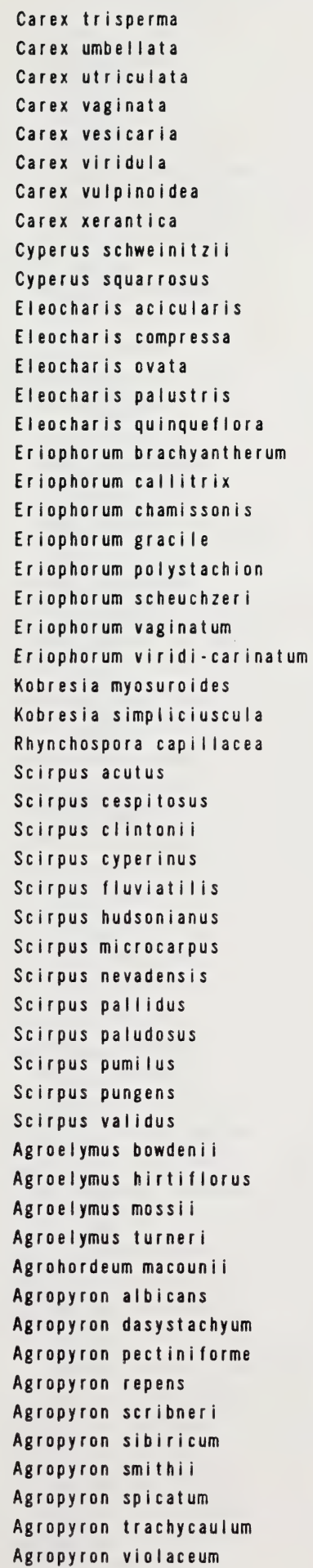

Carex trisperma

ENGLISH COMMON NAME

\begin{tabular}{|c|c|}
\hline three-seeded & sedge \\
\hline umbe I late & sedge \\
\hline beaked & sedge \\
\hline sheathed & $\operatorname{sedg} \theta$ \\
\hline blister & $\operatorname{sedge}$ \\
\hline green & $\operatorname{sedge}$ \\
\hline $10 x$ & sedge \\
\hline white-scaled & sedge \\
\hline sand & nut-grass \\
\hline awned & nut-grass \\
\hline needle & spike-rush \\
\hline flattened & spike-rush \\
\hline EngeImann's & spike-rush \\
\hline creeping & spike-rush \\
\hline few-flowered & spike-rush \\
\hline close-sheathed & cotton grass \\
\hline beautiful & cotton grass \\
\hline russett & cotton grass \\
\hline slender & cotton grass \\
\hline tall & cotton grass \\
\hline one-spike & cotton grass \\
\hline sheathed & cotton grass \\
\hline \multirow[t]{2}{*}{ thin-leaved } & cotton grass \\
\hline & bog-sedge \\
\hline simple & bog-sedge \\
\hline slender & beak-rush \\
\hline great & bulrush \\
\hline tufted & bulrush \\
\hline Clinton's & buIrush \\
\hline wool & grass \\
\hline river & bulrush \\
\hline alpine & cotton grass \\
\hline small-fruited & bulrush \\
\hline Nevada & bulrush \\
\hline green & bulrush \\
\hline prairie & bulrush \\
\hline dwarf & bulrush \\
\hline three-square & rush \\
\hline common great & bulrush \\
\hline
\end{tabular}

CARETRI ( )

CAREUMB ( )

CAREUTR ( )

CAREVAG ( )

CAREVES ( )

CAREVIR ( )

CAREVUL ( )

CAREXER ( )

CYPESCH ( )

CYPESQU ( )

ELEOACI ( )

ELEOCOM ( )

ELEOOVA ( )

ELEOPAL ( )

ELEOQUI ( )

ERIOBRA ( )

ERIOCAL ( )

ERIOCHA ( )

ERIOGRA ( )

ERIOPOL ( )

ERIOSCH ( )

ERIOVAG ( )

ERIOVIR ( )

KOBRMYO ( )

KOBRSIM ( )

RHYNCAP ( )

SCIRACU ( )

SCIRCES ( )

SCIRCLI ( )

SCIRCYP ( )

SCIRFLU ( )

SCIRHUD ( )

SCIRMIC ( )

SCIRNEV ( )

SCIRPAL ( )

SCIRPAU ( )

SCIRPUM ( )

SCIRPUN ( )

SCIRVAL ()

AGROBOW ( )

AGROHIR ( )

AGROMOS ( )

AGROTUR ( )

AGROMAC ( )

AGROALB ( )

AGRODAS ( )

AGROPEC ( )

AGROREP ( )

AGROSCR ( )

AGROSIB ( )

AGROSMI ( )

AGROSPI ( )

AGROTRA ( )

AGROVIO ( ) 
GRAMINEAE

GRAMINEAE

GRAMINEAE

GRAMINEAE

GRAMINEAE

GRAMINEAE

GRAMINEAE

GRAMINEAE

GRAMINEAE

GRAMINEAE

GRAMINEAE

GRAMINEAE

GRAMINEAE

GRAMINEAE

GRAMINEAE

GRAMINEAE

GRAMINEAE

GRAMINEAE

GRAMINEAE

GRAMINEAE

GRAMINEAE

GRAMINEAE

GRAMINEAE

GRAMINEAE

GRAMINEAE

GRAMINEAE

GRAMINEAE

GRAMINEAE

GRAMINEAE

GRAMINEAE

GRAMINEAE

GRAMINEAE

GRAMINEAE

GRAMINEAE

GRAMINEAE

GRAMINEAE

GRAM INEAE

GRAMINEAE

GRAM INEAE

GRAMINEAE

GRAM INEAE

GRAM INEAE

GRAM INEAE

GRAM INEAE

GRAMINEAE

GRAMINEAE

GRAMINEAE

GRAMINEAE

GRAMINEAE

GRAMINEAE

GRAMINEAE

GRAMINEAE

GRAMINEAE

GRAMINEAE
Agrostis exarata

Agrostis humilis

Agrostis mertensi

Agrostis scabra

Agrostis stolonifera

Agrostis thurberiana

Agrostis variabilis

Alopecurus aequalis

Alopecurus occidentalis

Alopecurus pratensis

Arctagrostis arundinacea

Aristida longiseta

Avena fatua

Avena sativa

Beckmannia syzigachne

Bouteloua gracilis

Bromus altissimus

Bromus anomalus

Bromus carinatus

Bromus ciliatus

Bromus commutatus

Bromus inermis

Bromus japonicus

Bromus mollis

Bromus secalinus

Bromus squarrosus

Bromus tectorum

Bromus vulgaris

Calamagrostis canadensis

Calamagrostis inexpansa

Calamagrostis Iapponica

Calamagrostis montanensis

Calamagrostis purpurascens

Calamagrostis rubescens

Calamagrostis stricta

Calamovilfa longifolia

Catabrosa aquatica

Cinna latifolia

Dactylis glomerata

Danthonia californica

Danthonia parryi

Danthonia spicata

Danthonia unispicata

Deschampsia cespitosa

Deschampsia elongata

Dicanthelium acuminatum

Dicanthelium leibergii

Dicanthelium oligosanthes

Digitaria ischaemum

Digitaria sanguinalis

Distichlis stricta

Echinochloa crusgalli

Elymus canadensis

Elymus glaucus spike

northern

hair

alpine

water

alpine

meadow

red

wild

cultivated

slough

blue

Canada

nodding

fringed

awnless

Japanese

field

downy

bluejoint

northern

plains

purple

pine

narrow

brook

drooping

California

Parry

poverty

one-spike

tufted

slender

sand

smooth

salt

barnyard

Canada

smooth redtop

bent grass

bent grass

grass

redtop

bent grass

redtop

foxtail

foxtail

foxtail

three-awn

oat

oat

grass

grama

brome

brome

brome

brome

brome

brome

chess

brome

brome

brome

chess

brome

reed grass

reed grass

reed grass

reed grass

reed grass

reed grass

sand grass

grass

wood-reed

orchard grass

oat grass

oat grass

oat grass

oat grass

hair grass

hair grass

millet

millet

millet

crabgrass

crabgrass

grass

grass

wild rye

wild rye
AGROEXA ( )

AGROHUM ( )

AGROMER ( )

AGROSCA ( )

AGROSTO ( )

AGROTHU ( )

AGROVAR ( )

ALOPAEQ ( )

ALOPOCC ( )

ALOPPRA ( )

ARCTARU ( )

ARISLON ( )

AVENFAT ( )

AVENSAT ( )

BECKSYZ ( )

BOUTGRA ( )

BROMALT ( )

BROMANO ( )

BROMCAR ( )

BROMCIL ( )

BROMCOM ( )

BROMINE ( )

BROMJAP ( )

BROMMOL ( )

BROMSEC ( )

BROMSQU ( )

BROMTEC ( )

BROMVUL ( )

CALACAN ( )

CALAINE ( )

CALALAP ( )

CALAMON ( )

CALAPUR ( )

CALARUB ( )

CALASTR ( )

CALALON ( )

CATAAQU ( )

CINNLAT ( )

DACTGLO ( )

DANTCAL ( )

DANTPAR ( )

DANTSPI ( )

DANTUNI ( )

DESCCES ( )

DESCELO ( )

DICAACU ( )

DICALEI ( )

DICAOLI ( )

DIGIISC ( )

DIGISAN ( )

DISTSTR ( )

ECHICRU ( )

ELYMCAN ( )

ELYMGLA ( ) 
GRAMINEAE

GRAMINEAE

GRAMINEAE

GRAMINEAE

GRAMINEAE

GRAMINEAE

GRAMINEAE

GRAM INEAE

GRAMINEAE

GRAMINEAE

GRAMINEAE

GRAM INEAE

GRAMINEAE

GRAMINEAE

GRAMINEAE

GRAM INEAE

GRAMINEAE

GRAM INEAE

GRAM INEAE

GRAM INEAE

GRAM INEAE

GRAM INEAE

GRAM INEAE

GRAM INEAE

GRAM INEAE

GRAM INEAE

GRAMINEAE

GRAM INEAE

GRAM INEAE

GRAMINEAE

GRAMINEAE

GRAM INEAE

GRAMINEAE

GRAMINEAE

GRAMINEAE

GRAMINEAE

GRAMINEAE

GRAM INEAE

GRAMINEAE

GRAMINEAE

GRAMINEAE

GRAMINEAE

GRAMINEAE

GRAMINEAE

GRAMINEAE

GRAMINEAE

GRAMINEAE

GRAMINEAE

GRAMINEAE

GRAMINEAE

GRAMINEAE

GRAMINEAE

GRAMINEAE

GRAMINEAE
Elymus innovatus

Elymus junceus

Elymus mollis

Elymus piperi

Elymus virginicus

Eragrost Is megastachya

Eremopyrum triticeum

Festuca baffinensis

Festuca brachyphylla

Festuca idahoensis

Festuca occidentalis

Festuca ovina

Festuca pratensis

Festuca rubra

Festuca saximontana

Festuca scabrella

Festuca subulata

Glyceria borealis

Glyceria elata

Glyceria grandis

Glyceria pulchella

Glyceria striata

Helictotrichon hooker $i$

Hierochloe alpina

Hierochloe odorata

Hordeum glaucum

Hordeum jubatum

Hordeum pusillum

Hordeum vulgare

Koeleria macrantha

Lolium multiflorum

Lolium perenne

Lolium persicum

Lolium temulentum

Melica smithii

Melica spectabilis

Melica subulata

Muhlentergia asperifolia

Muhlenbergia cuspidata

Muhlenbergia glomerata

Muhlenbergia racemosa

Muhlenbergia richardsonis

Munroa squarrosa

Oryzopsis asperifolia

Oryzopsis canadensis

Oryzopsis exigua

Oryzopsis hymenoides

Oryzopsis micrantha

Oryzopsis pungens

Panicum capillare

Panicum miliaceum

Phalaris arundinacea

Phalaris canariensis

Phleum commutatum hairy

Russian

American

giant

Virginia

skunk-grass

annual

bluebunch

sheep

meadow

red

sheep

rough

northern

tall

common tall

graceful

fowl

Hooker's

alpine

foxtail

little

cultivated

June

Italian

perennial

Persian

onion

Alaska onion

scratch

plains

bog

marsh

mat

false buffalo

white-grained mountain

Canadian

Iittle

Indian

little-seed

northern

witch

broomcorn

reed

mountain wild rye

wild rye

dune grass

wild rye

wild rye

wheat grass

fescue

fescue

fescue

fescue

fescue

fescue

fescue

fescue

fescue

fescue

manna grass

manna grass

manna grass

manna grass

manna grass

oat grass

sweet grass

sweet grass

barley

barley

barley

barley

grass

ryegrass

ryegrass

darnel

darnel

melic grass

grass

grass

grass

muhly

muhly

muhly

muhly

grass

rice grass

rice grass

rice grass

rice grass

rice grass

rice grass

grass

millet

canary grass

canary grass

timothy
ELYMINN ( )

ELYMJUN ( )

ELYMMOL ( )

ELYMPIP ( )

ELYMVIR ( )

ERAGMEG ( )

EREMTRI ( )

FESTBAF ( )

FESTBRA ( )

FESTIDA ( )

FESTOCC ( )

FESTOVI ( )

FESTPRA ( )

FESTRUB ( )

FESTSAX ( )

FESTSCA ( )

FESTSUB ( )

GLYCBOR ( )

GLYCELA ( )

GLYCGRA ( )

GLYCPUL ( )

GLYCSTR ( )

HELIHOO ( )

HIERALP ( )

HIERODO ( )

HORDGLA ( )

HORDJUB ( )

HORDPUS ( )

HORDVUL ( )

KOELMAC ( )

LOLIMUL ( )

LOLIPER ( )

LOLIPES ( )

LOLITEM ( )

MELISMI ( )

MELISPE ( )

MELISUB ( )

MUHLASP ( )

MUHLCUS ( )

MUHLGLO ( )

MUHLRAC ( )

MUHLRIC ( )

MUNRSQU ( )

ORYZASP ( )

ORYZCAN ( )

ORYZEXI ( )

ORYZHYM ( )

ORYZMIC ( )

ORYZPUN ( )

PANICAP ()

PANIMIL ()

PHALARU ()

PHALCAN ( )

PHLECOM ( ) 
GRAMINEAE

GRAM INEAE

GRAMINEAE

GRAM INEAE

GRAMINEAE

GRAMINEAE

GRAMINEAE

GRAM INEAE

GRAMINEAE

GRAMINEAE

GRAMINEAE

GRAMINEAE

GRAMINEAE

GRAMINEAE

GRAMINEAE

GRAMINEAE

GRAMINEAE

GRAMINEAE

GRAMINEAE

GRAMINEAE

GRAMINEAE

GRAMINEAE

GRAMINEAE

GRAMINEAE

GRAMINEAE

GRAMINEAE

GRAMINEAE

GRAMINEAE

GRAM INEAE

GRAM INEAE

GRAMINEAE

GRAMINEAE

GRAMINEAE

GRAMINEAE

GRAMINEAE

GRAMINEAE

GRAMINEAE

GRAMINEAE

GRAMINEAE

GRAMINEAE

GRAMINEAE

GRAMINEAE

GRAMINEAE

GRAMINEAE

GRAMINEAE

GRAMINEAE

GRAMINEAE

GRAMINEAE

GRAMINEAE

GRAM INEAE

GRAM INEAE

GRAMINEAE

GRAMINEAE

GRAMINEAE
Phleum pratense

Phragmites australis

Poa alpina

Poa annua

Poa arctica

Poa arida

Poa canbyi

Poa compressa

Poa cusickii

Poa epilis

Poa glauca

Poa gracillima

Poa interior

Poa juncifolia

Poa leptocoma

Poa lettermanii

Poa nemoralis

Poa nervosa

Poa nevadensis

Poa palustris

Poa pattersonii

Poa pratensis

Poa sandbergii

Poa stenantha

Polypogon monspeliensis

Puccinellia cusickii

Puccinellia distans

Puccinellia hauptiana

Puccinellia nuttalliana

Puccinellia pauciflora

Schedonnardus paniculatus

Schizachne purpurascens

Schizachyrium scoparium

Scolochloa festucacea

Secale cereale

Setaria glauca

Setaria viridis

Sitanion hystrix

Spartina gracilis

Spartina pectinata

Sphenopholis intermedia

Sphenopholis obtusata

Sporobolus cryptandrus

Sporobolus neglectus

Stipa columbiana

Stipa comata

Stipa curtiseta

Stipa richardsoni

Stipa spartea

Stipa viridula

Trisetum canescens

Trisetum cernuum

Trisetum flavescens

Trisetum montanum timothy

reed

alpine

annual

arctic

plains

Canby

Canada

early

skyline

Pacific

wood

a lkali

bog

Letterman's

wood

Wheeler's

Nevada

fowl

Patterson's

Kentucky

Sandberg

rabbitfoot

slender

Nuttall's

few-fl owered

tumble

little

spangletop

yellow

green

alkali

prairie

slender

prairie

sand

annual

Columbia

needle and thread

western porcupine

Richardson

porcupine

green

tall

nodding
PHLEPRA ( )

PHRAAUS ( )

POAALPI ( )

POAANNU ( )

POAARCT ( )

POAARID ( )

POACANB ( )

POACOMP ( )

POACUSI ( )

POAEPIL ( )

POAGLAU ( )

POAGRAC ( )

POAINTE ( )

POAJUNC ( )

POALEPT ( )

POALETT ( )

POANEMO ( )

POANERV ( )

POANEVA ( )

POAPALU ( )

POAPATT ( )

POAPRAT ( )

POASAND ( )

POASTEN ( )

POLYMON ( )

PUCCCUS ( )

PUCCDIS ( )

PUCCHAU ( )

PUCCNUT ( )

PUCCPAU ( )

SCHEPAN ( )

SCHIPUR ( )

SCHISCO ( )

SCOLFES ( )

SECACER ( )

SETAGLA ( )

SETAVIR ( )

SITAHYS ( )

SPARGRA ( )

SPARPEC ( )

SPHEINT ( )

SPHEOBT ( )

SPORCRY ( )

SPORNEG ( )

STIPCOL ( )

STIPCOM ( )

STIPCUR ( )

STIPRIC ( )

STIPSPA ( )

STIPVIR ( )

TRISCAN ( )

TRISCER

TRISFLA
TRISMON ( needle gras

trisetum

trisetum

trisetum

trisetum 


\begin{tabular}{|c|c|}
\hline GRAMINEAE & Trisetum spicatum \\
\hline GRAMINEAE & Trisetum wolfii \\
\hline GRAMINEAE & Triticum aestivum \\
\hline GRAMINEAE & Vahlodea atropurpurea \\
\hline GRAMINEAE & Vulpia octoflora \\
\hline GRAMINEAE & Zizania aquatica \\
\hline JUNCACEAE & Juncus albescens \\
\hline JUNCACEAE & Juncus alpinoarticulatus \\
\hline JUNCACEAE & Juncus balticus \\
\hline JUNCACEAE & Juncus biglumis \\
\hline JUNCACEAE & Juncus brevicaudatus \\
\hline JUNCACEAE & Juncus bufonius \\
\hline JUNCACEAE & Juncus castaneus \\
\hline JUNCACEAE & Juncus confusus \\
\hline JUNCACEAE & Juncus drummondii \\
\hline JUNCACEAE & Juncus ensifolius \\
\hline JUNCACEAE & Juncus filiformis \\
\hline JUNCACEAE & Juncus longistylis \\
\hline JUNCACEAE & Juncus mertensianus \\
\hline JUNCACEAE & Juncus nevadensis \\
\hline JUNCACEAE & Juncus nodosus \\
\hline JUNCACEAE & Juncus parryi \\
\hline JUNCACEAE & Juncus regelii \\
\hline JUNCACEAE & Juncus stygius \\
\hline JUNCACEAE & Juncus tenuis \\
\hline JUNCACEAE & Juncus torreyi \\
\hline JUNCACEAE & Juncus tracyi \\
\hline JUNCACEAE & Juncus vaseyi \\
\hline JUNCACEAE & Luzula acuminata \\
\hline JUNCACEAE & Luzula arcuata \\
\hline JUNCACEAE & Luzula groenlandica \\
\hline JUNCACEAE & Luzula hitchcockii \\
\hline JUNCACEAE & Luzula multiflora \\
\hline JUNCACEAE & Luzula parviflora \\
\hline JUNCACEAE & Luzula piperi \\
\hline JUNCACEAE & Luzula rufescens \\
\hline JUNCACEAE & Luzula spicata \\
\hline
\end{tabular}

spike
awnless
common
mountain
six-weeks
wild
white
alpine
wire
two-glumed
short-tail
toad
chestnut
few-flowered
Drummond's
equitant-leaved
thread
long-styled
slender-stemmed
Nevada
knotted
Parry's
marsh
slender
Torrey's
mud
big-head
reddish
spiked
fmooth
small-flowered
mountain
field

TRISSPI ( )

TRISWOL ( )

TRITAES ( )

VAHLATR ( )

VULPOCT ( )

ZIZAAQU ( )

JUNCALB ( )

JUNCALP ( )

JUNCBAL ( )

JUNCBIG ( )

JUNCBRE ( )

JUNCBUF ( )

JUNCCAS ( )

JUNCCON ( )

JUNCDRU ( )

JUNCENS ( )

JUNCFIL ( )

JUNCLON ( )

JUNCMER ( )

JUNCNEV ( )

JUNCNOD ( )

JUNCPAR ( )

JUNCREG ( )

JUNCSTY ( )

JUNCTEN ( )

JUNCTOR ( )

JUNCTRA ( )

JUNCVAS ( )

LUZUACU ( )

LUZUARC ( )

LUZUGRO ( )

LUZUHIT ( )

LUZUMUL ( )

LUZUPAR ( )

LUZUPIP ( )

LUZURUF ( )

LUZUSPI ( ) 


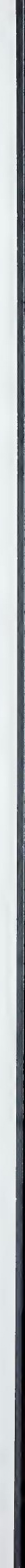




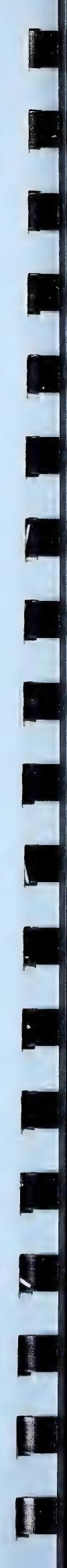


ADELANTHACEAE AMBLYSTEGIACEAE AMBLYSTEGIACEAE AMBLYSTEGIACEAE AMBLYSTEGIACEAE AMBLYSTEGIACEAE AMBLYSTEGIACEAE AMBLYSTEGIACEAE AMBLYSTEGIACEAE AMBLYSTEGIACEAE AMBLYSTEGIACEAE AMBLYSTEGIACEAE AMBLYSTEGIACEAE AMBLYSTEGIACEAE AMBLYSTEGIACEAE AMBLYSTEGIACEAE AMBLYSTEGIACEAE AMBLYSTEGIACEAE AMBLYSTEGIACEAE AMBLYSTEGIACEAE AMBLYSTEGIACEAE AMBLYSTEGIACEAE AMBLYSTEGIACEAE AMBLYSTEGIACEAE AMBLYSTEGIACEAE AMBLYSTEGIACEAE AMBLYSTEGIACEAE AMBLYSTEGIACEAE AMBLYSTEGIACEAE AMBLYSTEGIACEAE AMBLYSTEGIACEAE AMBLYSTEGIACEAE AMBLYSTEGIACEAE AMBLYSTEGIACEAE AMBLYSTEGIACEAE AMBLYSTEGIACEAE AMBLYSTEGIACEAE AMBLYSTEGIACEAE AMBLYSTEGIACEAE AMBLYSTEGIACEAE AMBLYSTEGIACEAE AMBLYSTEGIACEAE AMBLYSTEGIACEAE AMBLYSTEGIACEAE AMBLYSTEGIACEAE AMBLYSTEGIACEAE AMBLYSTEGIACEAE ANDREAEACEAE ANDREAEACEAE ANEURACEAE ANEURACEAE ANEURACEAE ANEURACEAE ANTHEL IACEAE
Odontoschisma denudatum

Amblystegium humile

Amblystegium noterophilum

Amblystegium riparium

Amblystegium serpens

Amblystegium tenax

Amblystegium varium

Calliergon cordifolium

Calliergon giganteum

Calliergon richardsoni $i$

Calliergon sarmentosum

Calliergon stramineum

Calliergon trifarium

Calliergonella cuspidata

Campy lium chrysophyl I um

Campylium halleri

Campylium hispidulum

Campylium polygamum

Campylium radicale

Campylium stellatum

Conardia compacta

Cratoneuron commutatum

Cratoneuron filicinum

Drepanocladus aduncus

brown

Drepanocladus capillifolius brown

Drepanocladus crassicostatus brown

Drepanocladus exannulatus brown

Drepanocladus fluitans brown

Drepanocladus lapponicus brown

Drepanocladus lycopodioides brown

Drepanocladus pseudostramineus brown

Drepanocladus revolvens brown

Drepanocladus sendtneri brown

Drepanocladus tundrae brown

Drepanocladus uncinatus brown

Drepanocladus vernicosus brown

Hygrohypnum alpestre

Hygrohypnum besti

Hygrohypnum duriusculum

Hygrohypnum Iuridum

Hygrohypnum molle

Hygrohypnum ochraceum

Hygrohypnum smithii

Hygrohypnum styriacum

Loeskypnum badium

Scorpidium scorpioides

Scorpidium turgescens

Andreaea nivalis

red

Andreaea rupestris

black

Aneura pinguis

Riccardia chamedryfolia

Riccardia latifrons

Riccardia multifida

Anthelia julacea liverwort

ODONDEN ( )

AMBLHUM ( )

AMBLNOT ( )

AMBLRIP ( )

AMBLSER ( )

AMBLTEN ( )

AMBLVAR ( )

CALLCOR ( )

CALLGIG ( )

CALLRIC ( )

CALLSAR ( )

CALLSTR ( )

CALLTRI ( )

CALLCUS ( )

CAMPCHR ( )

CAMPHAL ( )

CAMPHIS ()

CAMPPOL ( )

CAMPRAD ( )

CAMPSTE ( )

CONACOM ( )

CRATCOM ( )

CRATFIL ( )

DREPADU ( )

DREPCAP ( )

DREPCRA ( )

DREPEXA ( )

DREPFLU ( )

DREPLAP ( )

DREPLYC ( )

DREPPSE ( )

DREPREV ( )

DREPSEN ( )

DREPTUN ( )

DREPUNC ( )

DREPVER ( )

HYGRALP ( )

HYGRBES ( )

HYGRDUR ( )

HYGRLUR ( )

HYGRMOL ()

HYGROCH ( )

HYGRSMI ()

HYGRSTY ( )

LOESBAD ( )

SCORSCO ( )

SCORTUR ( )

ANDRNIV ( )

ANDRRUP ( )

ANEUPIN ( )

RICCCHA ()

RICCLAT ()

RICCMUL ( )

ANTHJUL ( ) 
ANTHELI ACEAE

ANTHOCEROTACEAE

ARNELLIACEAE

AULACOMNIACEAE

AULACOMN IACEAE

AULACOMN IACEAE

AULACOMN IACEAE

AYTONIACEAE

AYTONIACEAE

AYTONIACEAE

AYTONIACEAE

AYTONIACEAE

AYTONIACEAE

BARTRAMIACEAE

BARTRAMIACEAE

BARTRAMI ACEAE

BARTRAMIACEAE

BARTRAMIACEAE

BARTRAMIACEAE

BARTRAMIACEAE

BLASIACEAE

BRACHYTHECIACEAE

BRACHYTHECIACEAE

BRACHYTHECIACEAE

BRACHYTHECIACEAE

BRACHYTHECIACEAE

BRACHYTHECIACEAE

BRACHYTHECIACEAE

BRACHYTHECIACEAE

BRACHYTHECIACEAE

BRACHYTHECIACEAE

BRACHYTHECIACEAE

BRACHYTHECIACEAE

BRACHYTHECIACEAE

BRACHYTHECIACEAE

BRACHYTHECIACEAE

BRACHYTHECIACEAE

BRACHYTHECIACEAE

BRACHYTHECIACEAE

BRACHYTHECIACEAE

BRACHYTHECIACEAE

BRACHYTHECIACEAE

BRACHYTHECIACEAE

BRACHYTHECIACEAE

BRACHYTHECIACEAE

BRACHYTHECIACEAE

BRACHYTHECIACEAE

BRYACEAE

BRYACEAE

BRYACEAE

BRYACEAE

BRYACEAE

BRYACEAE

BRYACEAE
Anthelia juratzkana

Anthoceros punctatus

Arnellia fennica

liverwort

IIverwort

Aulacomnium acuminatum

Aulacomnium androgynum

Aulacomnium palustre

Aulacomnium turgidum

Asterella lindenbergiana

Asterella saccata

Mannia fragrans

Mannia pilosa

Mannia triandra

Reboulia hemisphaerica

Bartramia halleriana

Bartramia ithyphylla

Bartramia pomiformis

Conostomum tetragonum

Philonotis fontana

Philonotis marchica

Plagiopus oederiana

Blasia pusilla

Brachythecium campestre

Brachythecium collinum

Brachythecium frigidum

Brachythecium groenlandicum

Brachythecium hylotapetum

Brachythecium leibergi $i$

Brachythecium mildeanum

Brachythecium nelsonii

Brachythecium oedipodium

Brachythecium plumosum

Brachythecium populeum

Brachythecium reflexum

Brachythecium rivulare

Brachythecium rutabulum

Brachythecium salebrosum

Brachythecium turgidum

Brachythecium velutinum

Cirriphyllum cirrosum

Eurhynchium pulchellum

Homa l o thecium aeneum

Homal othecium nevadense

Homalothecium pinnatifidum

Scleropodium cespitans

Scleropodium obtusifolium

Tomenthypnum falcifolium

Tomenthypnum nitens

golden

moss

golden

Bryum algovicum

Bryum arcticum

Bryum argenteum

Bryum bicolor

Bryum caespiticium

Bryum calobryoides

Bryum calophyllum
ANTHJUR ( )

ANTHPUN ( )

ARNEFEN ( )

AULAACU ( )

AULAAND ( )

AULAPAL ( )

AULATUR ( )

ASTELIN ( )

ASTESAC ( )

MANNFRA ( )

MANNPIL ( )

MANNTRI ( )

REBOHEM ( )

BARTHAL ( )

BARTITH ( )

BARTPOM ( )

CONOTET ( )

PHILFON ( )

PHILMAR ( )

PLAGOED ( )

BLASPUS ( )

BRACCAM ( )

BRACCOL ( )

BRACFRI ( )

BRACGRO ( )

BRACHYL ( )

BRACLEI ( )

BRACMIL ( )

BRACNEL ( )

BRACOED ( )

BRACPLU ( )

BRACPOP ( )

BRACREF ( )

BRACRIV ( )

BRACRUT ( )

BRACSAL ( )

BRACTUR ( )

BRACVEL ( )

CIRRCIR ( )

EURHPUL ( )

HOMAAEN ( )

HOMANEV ( )

HOMAPIN ( )

SCLECES ( )

SCLEOBT ( )

TOMEFAL ( )

TOMENIT ( )

BRYUALG ( )

BRYUARC ( )

BRYUARG ( )

BRYUBIC ( )

BRYUCAE ( )

BRYUCAB ( )

BRYUCAL ( ) 
BRYACEAE

BRYACEAE

BRYACEAE

BRYACEAE

BRYACEAE

BRYACEAE

BRYACEAE

BRYACEAE

BRYACEAE

BRYACEAE

BRYACEAE

BRYACEAE

BRYACEAE

BRYACEAE

BRYACEAE

BRYACEAE

BRYACEAE

BRYACEAE

BRYACEAE

BRYACEAE

BRYACEAE

BRYACEAE

BRYACEAE

BRYACEAE

BRYACEAE

BRYACEAE

BRYACEAE

BRYACEAE

BRYACEAE

BRYACEAE

BRYACEAE

BRYACEAE

BRYACEAE

BRYACEAE

BRYACEAE

BRYACEAE

BRYACEAE

BRYACEAE

BRYACEAE

BRYACEAE

BRYACEAE

BRYACEAE

BRYACEAE

BUXBAUMIACEAE

BUXBAUMIACEAE

CALYPOGE JACEAE

CALYPOGE JACEAE

CALYPOGE JACEAE

CALYPOGE JACEAE

CALYPOGEJACEAE

CALYPOGE JACEAE

CATOSCOPIACEAE

CEPHALOZIACEAE

CEPHALOZIACEAE
Bryum capillare

Bryum cyclophyllum

Bryum intermedium

Bryum lisae var. cuspidatum

Bryum lonchocaulon

Bryum marratii

Bryum meesioides

Bryum muehlenbeckii

Bryum pallens

Bryum pallescens

Bryum pseudotriquetrum

Bryum purpurascens

Bryum schleicheri

Bryum stenotrichum

Bryum turbinatum

Bryum uliginosum

Bryum weigelii

Leptobryum pyriforme

Mielichnoferia macrocarpa

Plagiobryum demissum

Plagiobryum zierii

Pohlia andalusica

Pohlia annotina

Pohlia atropurpurea

Pohlia brevinervis

Pohlia bulbifera

Pohlia camptotrachela

Pohlia columbica

Pohlia cruda

Pohlia drumondii

Pohlia elongata

Pohlia filiformis

Pohlia filum

Pohlia longicollis

Pohlia nutans

Pohlia obtusifolia

Pohlia proligera

Pohlia sphagnicola

Pohlia vexans

Pohlia wahlenbergii

Rhodobryum ontariense

Rhodobryum roseum

Roellia roellii

Buxbaumia aphylla

Buxbaumia viridis

Calypogeja integristipula

Calypogeja muelleriana

Calypogeja neesiana

Calypogeja sphagnicola

Calypogeja suecica

Calypogeja trichomanis

Catoscopium nigritum

Cephalozia bicuspidata

Cephalozia catenulata copper wire

moss

liverwort

liverwort

liverwort

liverwort

liverwort

liverwort

liverwort

liverwort
BRYUCAP ( )

BRYUCYC ( )

BRYUINT ( )

BRYULIS ( )

BRYULON ( )

BRYUMAR ( )

BRYUMEE ( )

BRYUMUE ( )

BRYUPAL ( )

BRYUPAS ( )

BRYUPSE ( )

BRYUPUR ( )

BRYUSCH ( )

BRYUSTE ( )

BRYUTUR ( )

BRYUULI ( )

BRYUWEI ( )

LEPTPYF ( )

MIELMAC ( )

PLAGDEM ( )

PLAGZIE ( )

POHLAND ( )

POHLANN ( )

POHLATR ( )

POHLBRE ( )

POHLBUL ( )

POHLCAM ( )

POHLCOL ( )

POHLCRU ( )

POHLDRU ( )

POHLELO ( )

POHLFIL ( )

POHLFIU ( )

POHLLON ( )

POHLNUT ( )

POHLOBT ( )

POHLPRO ( )

POHLSPH ( )

POHLVEX ( )

POHLWAH ( )

RHODONT ( )

RHODROS ( )

ROELROE ( )

BUXBAPH ( )

BUXBVIR ( )

CALYINT ( )

CALYMUE ( )

CALYNEE ( )

CALYSPH ( )

CALYSUE ( )

CALYTRI ( )

CATONIG ( )

CEPHBIC ( )

CEPHCAT ( ) 
CEPHALOZIACEAE CEPHALOZIACEAE CEPHALOZIACEAE CEPHALOZIACEAE CEPHALOZIACEAE CEPHALOZIACEAE CEPHALOZIACEAE CEPHALOZIELLACEAE CEPHALOZIELLACEAE CEPHALOZIELLACEAE CEPHALOZIELLACEAE CLEVEACEAE

CLEVEACEAE

CLIMACIACEAE

CODONIACEAE

CONOCEPHALACEAE

DICRANACEAE

DICRANACEAE

DICRANACEAE

DICRANACEAE

DICRANACEAE

DICRANACEAE

DICRANACEAE

DICRANACEAE

DICRANACEAE

DICRANACEAE

DICRANACEAE

DICRANACEAE

DICRANACEAE

DICRANACEAE

DICRANACEAE

DICRANACEAE

DICRANACEAE

DICRANACEAE

DICRANACEAE

DICRANACEAE

DICRANACEAE

DICRANACEAE

DICRANACEAE

DICRANACEAE

DICRANACEAE

DICRANACEAE

DICRANACEAE

DICRANACEAE

DICRANACEAE

DICRANACEAE

DICRANACEAE

DICRANACEAE

DICRANACEAE

DICRANACEAE

DICRANACEAE

DICRANACEAE

DICRANACEAE

DICRANACEAE
Cephalozia connivens

Cephalozia lunulifolia

Cephalozia macounii

Cephalozia pleniceps

Cladopodiella fluitans

Hygrobiella laxifolia

Pleuroclada albescens

Cephaloziella divaricata

Cephaloziella elachista

Cephalozlella hampeana

Cephaloziella rubella

Athalamia hyalina

Sauteria alpina

Climacium dendroides

Fossombronia macouni $i$

Conocephalum conicum

Aongstroemia longipes

Cnestrum alpestre

Cnestrum glaucescens

Cnestrum schistii

Cynodontium strumiferum

Cynodontium tenellum

Dichodontium olympicum

Dichodontium pellucidum

Dicranella cerviculata

Dicranella crispa

Dicranella grevilleana

Dicranella heteromalla

Dicranella palustris

Dicranella schreberiana

Dicranella subulata

Dicranella varia

Dicranoweisia crispula

Dicranum acutifolium

Dicranum angustum

Dicranum bonjeani

Dicranum brevifolium

Dicranum elongatum

Dicranum flagellare

Dicranum fragilifolium

Dicranum fuscescens

Dicranum groenlandicum

Dicranum majus

Dicranum muehlenbecki

Dicranum ontariense

Dicranum pallidisetum

Dicranum polysetum

Dicranum scoparium

Dicranum spadiceum

Dicranum tauricum

Dicranum undulatum

Kiaeria blyttii

Kiaeria falcata

Kiaeria starkei liverwort

liverwort

liverwort

liverwort

liverwort

liverwort

liverwort

liverwort

liverwort

IIverwort

liverwort

liverwort

liverwort

liverwort

cushion

cushion

cushion

cushion

cushion

cushion

cushion

cushion

cushion

cushion

cushion

cushion

cushion

cushion

broom

cushion

cushion

cushion
CEPHCON ( )

CEPHLUN ( )

CEPHMAC ( )

CEPHPLE ( )

CLADFLU ( )

HYGRLAX ( )

PLEUALB ( )

CEPHDIV ( )

CEPHELA ( )

CEPHHAM ( )

CEPHRUB ( )

ATHAHYA ( )

SAUTALP ( )

CLIMDEN ( )

FOSSMAC ( )

CONOCON ( )

AONGLON ( )

CNESALP ( )

CNESGLA ( )

CNESSCH ( )

CYNOSTR ( )

CYNOTEN ( )

DICHOLY ( )

DICHPEL ( )

DICRCER ( )

DICRCRI ( )

DICRGRE ( )

DICRHET ( )

DICRPAU ( )

DICRSCH ( )

DICRSUB ( )

DICRVAR ( )

DICRCRS ( )

DICRACU ( )

DICRANG ( )

DICRBON ( )

DICRBRE ( )

DICRELO ( )

DICRFLA ( )

DICRFRA ( )

DICRFUS ( )

DICRGRO ( )

DICRMAJ ( )

DICRMUE ()

DICRONT ( )

DICRPAL ( )

DICRPOL ( )

DICRSCO ()

DICRSPA ()

DICRTAU ( )

DICRUND ( )

KIAEBLY ( )

KIAEFAL ( )

KIAESTA ( ) 
DICRANACEAE

DICRANACEAE

DICRANACEAE

DICRANACEAE

DICRANACEAE

DISCELIIACEAE

DITRICHACEAE

DITRICHACEAE

DITRICHACEAE

DITRICHACEAE

DITRICHACEAE

DITRICHACEAE

DITRICHACEAE

DITRICHACEAE

ENCALYPTACEAE

ENCALYPTACEAE

ENCALYPTACEAE

ENCALYPTACEAE

ENCALYPTACEAE

ENCALYPTACEAE

ENCALYPTACEAE

ENCALYPTACEAE

ENCALYPTACEAE

ENCALYPTACEAE

ENCALYPTACEAE

ENCALYPTACEAE

ENCALYPTACEAE

ENTODONTACEAE

ENTODONTACEAE

ENTODONTACEAE

ENTODONTACEAE

FISSIDENTACEAE

FISSIDENTACEAE

FISSIDENTACEAE

FISSIDENTACEAE

FISSIDENTACEAE

FONTINALACEAE

FONT INALACEAE

FONT INALACEAE

FONT INALACEAE

FONTINALACEAE

FONTINALACEAE

FONTINALACEAE

FUNARI ACEAE

FUNARIACEAE

FUNARIACEAE

FUNARI ACEAE

FUNARI ACEAE

GEOCALYCACEAE

GEOCALYCACEAE

GRIMMIACEAE

GRIMMIACEAE

GRIMMIACEAE

GRIMMIACEAE
Oncophorus virens

Oncophorus wahlenbergii

Oreas martiana

Paral eucobryum enerve

Paraleucobryum longifolium

Discelium nudum

Ceratodon purpureus

Distichium capillaceum

Distichium inclinatum

Ditrichum crispatissimum

Ditrichum flexicaule

Ditrichum montanum

Saelania glaucescens

Trichodon cylindricus

Bryobrittonia longipes

Encalypta affinis

Encalypta alpina

Encalypta brevicolla

Encalypta brevipes

Encalypta ciliata

Encalypta intermedia

Encalypta longicolla

Encalypta mutica

Encalypta procera

Encalypta rhaptocarpa

Encalypta spathulata

Encalypta vulgaris

Entodon brevisetus

Entodon cladorrhizans

Entodon concinnus

Entodon schleicheri

Fissidens adianthoides

Fissidens bryoides

Fissidens grandifrons

Fissidens limbatus

Fissidens osmundoides

Dichelyma falcatum

Fontinalis antipyretica

Fontinalis dalecarlica

Fontinalis howellii

Fontinalis hypnoides

Fontinalis neomexicana

fontinalis novae-angliae

Funaria americana

Funaria hygrometrica

Funaria muhlenbergi

Physcomitrium hookeri

Physcomitrium pyriforme

Geocalyx graveolens

Harpanthus flotovianus

Coscinodon calyptratus

Dryptodon patens

Grimmia affinis

Grimmia anodon
ONCOVIR ( )

ONCOWAH ( )

OREAMAR ( )

PARAENE ( )

PARALON ( )

DISCNUD ( )

CERAPUR ( )

DISTCAP ( )

DISTINC ( )

DITRCRI ( )

DITRFLE ( )

DITRMON ( )

SAELGLA ( )

TRICCYL ( )

BRYOLON ( )

ENCAAFF ( )

ENCAALP ( )

ENCABRC ( )

ENCABRE ( )

ENCACIL ( )

ENCAINT ( )

ENCALON ( )

ENCAMUT ( )

ENCAPRO ( )

ENCARHA ( )

ENCASPA ( )

ENCAVUL ( )

ENTOBRE ( )

ENTOCLA ( )

ENTOCON ( )

ENTOSCH ( )

FISSADI ( )

FISSBRY ( )

FISSGRA ( )

FISSLIM ( )

FISSOSM ( )

DICHFAL ( )

FONTANT ( )

FONTDAL ( )

FONTHOW ( )

FONTHYP ( )

FONTNEO ( )

FONTNOV ( )

FUNAAME ( )

FUNAHYG ( )

FUNAMUH ( )

PHYSHOO ( )

PHYSPYR ( )

liverwort

GEOCGRA ( )

HARPFLO ( )

COSCCAL ( )

DRYPPAT ( )

GRIMAFF ( )

GRIMANO ( ) 
GRIMMIACEAE

GRIMMIACEAE

GRIMMIACEAE

GRIMMIACEAE

GRIMMIACEAE

GRIMMIACEAE

GRIMMIACEAE

GR IMMIACEAE

GR IMMIACEAE

GR IMMIACEAE

GRIMMIACEAE

GRIMMIACEAE

GRIMMIACEAE

GRIMMIACEAE

GRIMMIACEAE

GRIMMIACEAE

GRIMMIACEAE

GRIMMIACEAE

GRIMMIACEAE

GRIMMIACEAE

GRIMMIACEAE

GR IMMIACEAE

GR IMMIACEAE

GR IMMIACEAE

GRIMMIACEAE

GR IMMIACEAE

GRIMMIACEAE

GRIMMIACEAE

GYMNOMITRIACEAE

GYMNOMITRIACEAE

GYMNOMITRIACEAE

GYMNOMITRIACEAE

GYMNOMITRIACEAE

GYMNOMITRIACEAE

GYMNOMITRIACEAE

HEDWIGIACEAE

HYLOCOMIACEAE

HYLOCOMIACEAE

HYLOCOMIACEAE

HYLOCOMIACEAE

HYLOCOMIACEAE

HYLOCOMIACEAE

HYLOCOMIACEAE

HYLOCOMIACEAE

HYPNACEAE

HYPNACEAE

HYPNACEAE

HYPNACEAE

HYPNACEAE

HYPNACEAE

HYPNACEAE

HYPNACEAE

HYPNACEAE

HYPNACEAE
Grimmia anomala

Grimmia donniana

Grimmia elatior

Grimmia elongata

Grimmia heterophylla

Grimmia incurva

Grimmia montana

Grimmia pilifera

Grimmia plagiopodia

Grimmia pulvinata

Grimmia tenerrima

Grimmia torquata

Grimmia trichophylla

Jaffueliobryum raui

Jaffueliobryum wrightii

Rhacomitrium aciculare

Rhacomitrium canescens

Rhacomitrium elongatum

Rhacomitrium fasciculare

Rhacomitrium heterostichum

Rhacomitrium Ianuginosum

Rhacomitrium sudeticum

Rhacomitrium varium

Schistidium agassizi

Schistidium apocarpum

Schistidium rivulare

Schistidium tenerum

Scouleria aquatica

Gymnomitrion concinnatum

Gymnomitrion corallioides

Marsupella brevissima

Marsupella emarginata

Marsupella revoluta

Marsupella sparsifolia

Marsupella sphacelata

Hedwigia ciliata

Hylocomium pyreniacum

Hylocomium splendens

Pleurozium schreberi

Rhytidiadelphus loreus

Rhytidiadelphus squarrosus

Rhytidiadelphus subpinnatus

stair-step

Schreber's

pipecleaner

pipecleaner

pipecleaner

Rhytidiadelphus triquetrus

red-stemmed pipecleaner

liverwort

liverwort

I iverwort

liverwort

liverwort

Iiverwort

Rhytidiopsis robusta

Herzogiella seligeri

Herzogiella turfacea

Homomallium adnatum

Hypnum bambergeri

Hypnum callichroum

Hypnum cupressiforme

Hypnum I indbergi i

Hypnum pallescens

Hypnum pratense

Hypnum procerrimum
GRIMANO ( )

GRIMDON ( )

GRIMELA ( )

GRIMELO ( )

GRIMHET ( )

GRIMINC ( )

GRIMMON ( )

GRIMPIL ( )

GRIMPLA ( )

GRIMPUL ( )

GRIMTER ( )

GRIMTOR ( )

GRIMTRI ( )

JAFFRAU ( )

JAFFWR I ( )

RHACACI ( )

RHACCAN ( )

RHACELO ( )

RHACFAS ( )

RHACHET ( )

RHACLAN ( )

RHACSUD ( )

RHACVAR ( )

SCHIAGA ( )

SCHIAPO ( )

SCHIRIV ( )

SCHITEN ( )

SCOUAQU ( )

GYMNCON ( )

GYMNCOR ( )

MARSBRE ( )

MARSEMA ( )

MARSREV ( )

MARSSPA ( )

MARSSPH ( )

HEDWCIL ( )

HYLOPYR ( )

HYLOSPL ( )

PLEUSCH ( )

RHYTLOR ( )

RHYTSQU ( )

RHYTSUB ( )

RHYTTRI ( )

RHYTROB ( )

HERZSEL ( )

HERZTUR ( )

HOMOADN ( )

HYPNBAM ( )

HYPNCAL ( )

HYPNCUP ( )

HYPNLIN ( )

HYPNPAL ( )

HYPNPRA ( )

HYPNPRO ( ) 
HYPNACEAE

HYPNACEAE

HYPNACEAE

HYPNACEAE

HYPNACEAE

HYPNACEAE

HYPNACEAE

HYPNACEAE

HYPNACEAE

HYPNACEAE

HYPNACEAE

HYPNACEAE

JUBULACEAE

JUNGERMANN I ACEAE

JUNGERMANN IACEAE

JUNGERMANN IACEAE

JUNGERMANN IACEAE

JUNGERMANN IACEAE

JUNGERMANN IACEAE

JUNGERMANN I ACEAE

JUNGERMANN IACEAE

JUNGERMANN I ACEAE

JUNGERMANN I ACEAE

JUNGERMANN I ACEAE

JUNGERMANN I ACEAE

JUNGERMANN IACEAE

JUNGERMANN IACEAE

JUNGERMANN I ACEAE

JUNGERMANN I ACEAE

JUNGERMANN I ACEAE

JUNGERMANN I ACEAE

JUNGERMANN I ACEAE

JUNGERMANN IACEAE

JUNGERMANN IACEAE

JUNGERMANN I ACEAE

JUNGERMANN I ACEAE

JUNGERMANN I ACEAE

JUNGERMANN I ACEAE

JUNGERMANN I ACEAE

JUNGERMANN I ACEAE

JUNGERMANN I ACEAE

JUNGERMANN I ACEAE

JUNGERMANN I ACEAE

JUNGERMANN I ACEAE

JUNGERMANN I ACEAE

JUNGERMANN IACEAE

JUNGERMANN I ACEAE

JUNGERMANN IACEAE

JUNGERMANN IACEAE

JUNGERMANN I ACEAE

JUNGERMANN I ACEAE

JUNGERMANN I ACEAE

JUNGERMANN I ACEAE

JUNGERMANN I ACEAE
Hypnum recurvatum

Hypnum revolutum

Hypnum vaucher $i$

I sopterygium pulchellum

Orthothecium chryseum

Orthothecium intricatum

Orthothecium strictum

Platydictya jungermannioides

Platydictya minutissimum

Platygyrium repens

Ptilium crista-castrensis

Pylaisiella polyantha

Frullania inflata

Anastrophyllum helleranum

Anastrophyllum michauxi

Anastrophyllum minutum

Barbilophozia attenuata

Barbilophozia barbata

Barbilophozia binsteadii

Barbilophozia floerkil

Barbilophozia hatcheri

Barbilophozia kunzeana

Barbilophozia lycopodioides

Barbilophozia quadriloba

Chandonanthus setiformis

Gymnocolea inflata

Jamesoniella autumnalis

Jungermannia atrovirens

Jungermannia exsertifolia

Jungermannia hyalina

Jungermannia leiantha

Jungermannia obovata

Jungermannia polaris

Jungermannia pumila

Jungermannia rubra

Jungermannia sphaerocarpa

Lophozia alpestris

Lophozia ascendens

Lophozia badensis

Lophozia bantriensis

Lophozia collaris

Lophozia excisa

Lophozia gillmanii

Lophozia grandiretis

Lophozia guttulata

Lophozia heterocolpos

Lophozia incisa

Lophozia laxa

Lophozia longidens

Lophozia obtusa

Lophozia rutheana

Lophozia ventricosa

Lophozia wenzelii

Mylia anomala knight's plume moss

liverwort

Iiverwort

liverwort

liverwort

Iiverwort

liverwort

I iverwort

liverwort

liverwort

liverwort

liverwort

Iiverwort

I iverwort

liverwort

liverwort

liverwort

liverwort

liverwort

I iverwort

I iverwort

liverwort

I iverwort

liverwort

liverwort

liverwort

liverwort

I iverwort

I iverwort

l iverwort

liverwort

I iverwort

liverwort

I iverwort

I iverwort

I iverwort

I iverwort

liverwort

I iverwort

Iiverwort

liverwort

liverwort
HYPNREC ( )

HYPNREV ( )

HYPNVAU ( )

ISOPPUL ( )

ORTHCHR ( )

ORTHINT ( )

ORTHSTR ( )

PLATJUN ( )

PLATMIN ( )

PLATREP ( )

PTILCRI ()

PYLAPOL ( )

FRULINF ( )

ANASHEL ( )

ANASMIC ( )

ANASMIN ( )

BARBATT ()

BARBBAR ( )

BARBBIN ( )

BARBFLO ( )

BARBHAT ( )

BARBKUN ( )

BARBLYC ( )

BARBQUA ( )

CHANSET ( )

GYMNINF ( )

JAMEAUT ( )

JUNGATR ( )

JUNGEXS ( )

JUNGHYA ( )

JUNGLEI ( )

JUNGOBO ( )

JUNGPOL ( )

JUNGPUM ( )

JUNGRUB ( )

JUNGSPH ( )

LOPHALP ( )

LOPHASC ( )

LOPHBAD ( )

LOPHBAN ( )

LOPHCOL ( )

LOPHEXC ( )

LOPHGIL ( )

LOPHGRA ( )

LOPHGUT ( )

LOPHHET ( )

LOPHINC ( )

LOPHLAX ( )

LOPHLON ( )

LOPHOBT ( )

LOPHRUT ( )

LOPHVEN ( )

LOPHWEN ( )

MYLIANO ( ) 


\section{JUNGERMANN I ACEAE JUNGERMANN I ACEAE JUNGERMANN IACEAE JUNGERMANN I ACEAE JUNGERMANN I ACEAE JUNGERMANN I ACEAE JUNGERMANN I ACEAE JUNGERMANN I ACEAE \\ LEPIDOZIACEAE LEPIDOZIACEAE \\ LESKEACEAE \\ LESKEACEAE \\ LESKEACEAE \\ LESKEACEAE \\ LESKEACEAE \\ LESKEACEAE \\ LESKEACEAE \\ LESKEACEAE \\ LESKEACEAE}

LOPHOCOLEACEAE LOPHOCOLEACEAE LOPHOCOLEACEAE LOPHOCOLEACEAE LOPHOCOLEACEAE MARCHANTIACEAE MARCHANTIACEAE MARCHANT IACEAE MEESIACEAE MEESIACEAE MEESIACEAE MEES IACEAE MEESIACEAE

MN I ACEAE

MN I ACEAE

MNI ACEAE

MN I ACEAE

MN I ACEAE

MN I ACEAE

MN IACEAE

MNIACEAE

MN I ACEAE

MN I ACEAE

MN I ACEAE

MN I ACEAE

MN I ACEAE

MN I ACEAE

MN I ACEAE

MNIACEAE

MN I ACEAE

MN I ACEAE

MN I ACEAE NE CKERACEAE NECKERACEAE NECKERACEAE

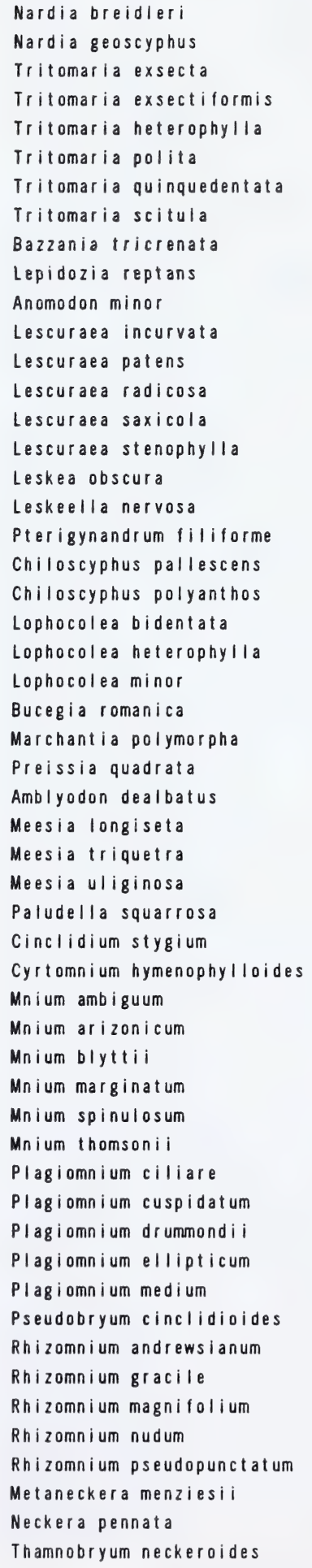

Iiverwort

liverwort

liverwort

liverwort

liverwort

liverwort

liverwort

liverwort

liverwort

liverwort

liverwort

liverwort

liverwort

liverwort

liverwort

Iiverwort

liverwort
NARDBRE ( )

NARDGEO ( )

TRITEXA ( )

TRITEXS ( )

TRITHET ( )

TRITPOL ( )

TRITQUI ( )

TRITSCI ( )

BAZZTRI ( )

LEPIREP ( )

ANOMMIN ( )

LESCINC ( )

LESCPAT ( )

LESCRAD ( )

LESCSAX ( )

LESCSTE ( )

LESKOBS ( )

LESKNER ( )

PTERFIL ( )

CHILPAL ( )

CHILPOL ( )

LOPHBID ( )

LOPHHEY ( )

LOPHMIN ( )

BUCEROM ( )

MARCPOL ( )

PREIQUA ( )

AMBLDEA ( )

MEESLON ( )

MEESTRI ( )

MEESULI ( )

PALUSQU ( )

CINCSTY ( )

CYRTHYM ( )

MNIUAMB ( )

MNIUARI ( )

MNIUBLY ( )

MNIUMAR ( )

MNIUSPI ( )

MNIUTHO ( )

PLAGCIL ( )

PLAGCUS ( )

PLAGDRU ( )

PLAGELL ( )

PLAGMED ( )

PSEUCIN ( )

RHIZAND ( )

RHIZGRA ( )

RHIZMAG ( )

RHIZNUD ( )

RHIZPSE ( )

METAMEN ( )

NECKPEN ( )

THAMNEC ( ) 
FAMILY

LATIN NAME

ORTHOTRICHACEAE CRTHOTRICHACEAE ORTHOTRICHACEAE ORTHOTRICHACEAE ORTHOTRICHACEAE ORTHOTRICHACEAE ORTHOTRICHACEAE ORTHOTRICHACEAE ORTHOTRI CHACEAE ORTHOTRI CHACEAE ORTHOTRICHACEAE ORTHOTRI CHACEAE ORTHOTRI CHACEAE

PALLAVICINIACEAE

PALLAVICINIACEAE

PELLIACEAE

PELLIACEAE

PELLIACEAE

PLAGIOCHILACEAE

PLAGIOTHECIACEAE

PLAG IOTHECIACEAE

POLYTRICHACEAE

POLYTRICHACEAE

POLYTRICHACEAE

POLYTRICHACEAE

POLYTRICHACEAE

POLYTRICHACEAE

POLYTRICHACEAE

POLYTRICHACEAE

POLYTRI CHACEAE

POLYTRICHACEAE

POLYTRICHACEAE

POLYTRI CHACEAE

POLYTRICHACEAE

POLYTRICHACEAE

POLYTRICHACEAE

PORELLACEAE

PORELLACEAE

PORELLACEAE

POTTIACEAE

POTTIACEAE

POTTIACEAE

POTTIACEAE

POTTIACEAE

POTTIACEAE

POTTIACEAE

POTTIACEAE POTTIACEAE POTTIACEAE POTTIACEAE POTTIACEAE POTTIACEAE

Ulota curvifolia

Moerckia blyttii

Pellia epiphylla

Pellia neesiana

Aloina rigida

ferruginascens

recurvirostrum
Orthotrichum affine

Orthotrichum alpestre

Orthotrichum anomalum

Orthotrichum laevigatum

Orthotrichum obtusifolium

Orthotrichum pallens

Orthotrichum pellucidum

Orthotrichum pulchellum

Orthotrichum pumilum

Orthotrichum pylaisi

Orthotrichum rupestre

Orthotrichum speciosum

Moerckia hibernica

Pellia endivifolia

Plagiochila asplenioides

Plagiothecium denticulatum

Plagiothecium laetum

Atrichum selwynii

Atrichum undulatum

Oligotrichum aligerum

Oligotrichum hercynicum

Oligotrichum parallelum

Pogonatum dentatum

Pogonatum urnigerum

Polytrichum alpinum

Polytrichum commune

Polytrichum juniperinum

Polytrichum longisetum

Polytrichum lyallii

Polytrichum piliferum

Polytrichum sexangulare

Polytrichum strictum

Porella cordaeana

Porella navicularis

Porella platyphylla

Aloina brevirostris

Anoectangium sendtnerianum

Barbula convoluta

Barbula unguiculata

Bryoerythrophy I I um

Bryoerythrophyllum

Desmatodon cernuus

liverwort

liverwort

liverwort

liverwort

liverwort

liverwort

undulated crane's bill

Hercynian

hair-like

urn-like

hair cap

common

juniper

slender

hair cap

awned

northern

slender

CODE

CHECK

ORTHAFF ( )

ORTHALP ( )

ORTHANO ( )

ORTHLAE ( )

ORTHOBT ( )

ORTHPAL ( )

ORTHPEL ( )

ORTHPUL ( )

ORTHPUM ( )

ORTHPYL ( )

ORTHRUP ( )

ORTHSPE ( )

ULOTCUR ( )

MOERBLY ( )

MOERHIB ( )

PELLEND ( )

PELLEPI ( )

PELLNEE ( )

PLAGASP ( )

PLAGDEN ( )

PLAGLAE ( )

ATRISEL ( )

ATRIUND ( )

OLIGALI ( )

OLIGHER ( )

OLIGPAR ( )

POGODEN ( )

POGOURN ( )

POLYALP ( )

POLYCOM ( )

POLYJUN ( )

POLYLOG ( )

POLYLYA ( )

POLYPIL ( )

POLYSEX ( )

POLYSTR ( )

PORECOR ( )

PORENAV ( )

POREPLA ( )

ALOIBRE ( )

ALOIRIG ( )

ANOESEN ( )

BARBCON ( )

BARBUNG ( )

BRYOFER ( )

BRYOREC ( )

DESMCER ( )

DESMGUE ( )

DESMHEI ( )

DESMLAT ( )

DESMLAU ( )

DESMLEU ( ) 
POTTIACEAE

POTTIACEAE

POTTIACEAE

POTTIACEAE

POTTIACEAE

POTTIACEAE

POTTIACEAE

POTTIACEAE

POTTIACEAE

POTTIACEAE

POTTIACEAE

POTTIACEAE

POTTIACEAE

POTTIACEAE

POTTIACEAE

POTTIACEAE

POTTIACEAE

POTTIACEAE

POTTIACEAE

POTTIACEAE

POTTIACEAE

POTTIACEAE

POTTIACEAE

POTTIACEAE

POTTIACEAE

POTTIACEAE

POTTIACEAE

POTTIACEAE

POTTIACEAE

PSEUDOLEPICOLEACEAE

PTILIDIACEAE

PTILIDIACEAE

RADULACEAE

RHABDOWE ISIACEAE

RHABDOWE ISIACEAE

RHYTIDIACEAE

RICCIACEAE

RICCIACEAE

RICCIACEAE

SCAPAN IACEAE

SCAPAN IACEAE

SCAPAN IACEAE

SCAPAN I ACEAE

SCAPAN IACEAE

SCAPANIACEAE

SCAPANIACEAE

SCAPAN IACEAE

SCAPAN IACEAE

SCAPAN IACEAE

SCAPAN IACEAE

SCAPAN IACEAE

SCAPAN IACEAE

SCAPAN I ACEAE

SCAPAN IACEAE
Desmatodon obtusifolius

Desmatodon randi $i$

Vesmatodon systylius

Didymodon acutus

Didymodon asperifolius

Didymodon fallax

Didymodon johansenii

Didymodon nigrescens

Didymodon rigidulus

Didymodon subandreacoides

Didymodon tophaceus

Gymnostomum aeruginosum

Gymnostomum recurvirostrum

Oxystegus tenuirostris

Phascum cuspidatum

Pottia nevadensis

Pottia truncata

Pterygoneurum ovatum

Pterygoneurum subsessile

Stegonia latifolia

Tortella fragilis

Tortella inclinata

Tortella tortuosa

Tortula caninervis

Tortula mucronifolia

Tortula norvegica

Tortula princeps

Tortula ruralis

Weissia controversa

Blepharostoma trichophyllum

Iiverwort

Ptilidium ciliare

Ptilidium pulcherrimum

Radula complanata

liverwort

liverwort

liverwort

Amphidium lapponicum

Amphidium mougeoti $\mathrm{i}$

Rhytidium rugosum

pipecleaner

moss

Riccia beyrichiana

Riccia cavernosa

Ricciocarpos natans

Diplophyllum albicans

Diplophyllum taxifolium

Scapania apiculata

Scapania brevicaulis

Scapania carinthiaca

Scapania curta

Scapania cuspiduligera

Scapania degenii

Scapania glaucocephala

Scapania irrigua

Scapania mucronata

Scapania nemorosa

Scapania paludicola

Scapania paludosa

Scapania subalpina
DESMOBT ( )

DESMRAN ( )

DESMSYS ( )

DIDYACU ( )

DIDYASP ( )

DIDYFAL ( )

DIDYJOH ( )

DIDYNIG ( )

DIDYRIG ( )

DIDYSUB ( )

DIDYTOP ( )

GYMNAER ( )

GYMNREC ( )

OXYSTEN ( )

PHASCUS ( )

POTTNEY ( )

POTTTRU ( )

PTEROVA ( )

PTERSUB ( )

STEGLAT ( )

TORTFRA ( )

TORTINC ( )

TORTTOR ( )

TORTCAN ( )

TORTMUC ( )

TORTNOR ( )

TORTPRI ( )

TORTRUR ( )

WEISCON ( )

BLEPTRI ( )

PTILCIL ( )

PTILPUL ( )

RADUCOM ( )

AMPHLAP ( )

AMPHMOU ( )

RHYTRUG ( )

RICCBEY ( )

RICCCAV ( )

RICCNAT ( )

DIPLALB ()

DIPLTAX ()

SCAPAPI ()

SCAPBRE ( )

SCAPCAR ( )

SCAPCUR ( )

SCAPCUS ( )

SCAPDEG ( )

SCAPGLA ( )

SCAPIRR ( )

SCAPMUC ( )

SCAPNEM ()

SCAPPAL ( )

SCAPPAU ()

SCAPSUB ( ) 
SCAPANIACEAE SCHISTOSTEGACEAE

Scapania undulata

Schistostega pennata

Blindia acuta

Seligeria calcarea

Seligeria campylopoda

Seligeria donniana

Seligeria subimersa

Seligeria tristichoides

Sphagnum angustifolium

Sphagnum balticum

Sphagnum capillifolium

Sphagnum centrale

Sphagnum compactum

Sphagnum contortum

Sphagnum fallax

Sphagnum fimbriatum

Sphagnum fuscum

Sphagnum girgensohni i

Sphagnum jenseni $i$

Sphagnum lindbergi i

Sphagnum magellanicum

Sphagnum majus

Sphagnum obtusum

Sphagnum riparium

Sphagnum russowi i

Sphagnum squarrosum

Sphagnum subsecundum

Sphagnum teres

Sphagnum warnstorfii

Sphagnum wulfianum

Splachnum ampullaceum

Splachnum luteum

Splachnum rubrum

Splachnum sphaericum

Splachnum vasculosum

Tayloria acuminata

Tayloria froehlichiana

Tayloria hornschuchii

Tayloria lingulata

Tayloria serrata

Tayloria splachnoides

Tetraplodon angustatus

Tetraplodon mnioides

Tetraplodon urceolatus

Voitia nivalis

Tetraphis pellucida

Claopodium bolanderi

Haplocladium microphyl l um

Haplocladium virginianum

Helodium blandowi $i$

Heterocladium dimorphum

Myurella julacea

Myurella sibirica

Myurella tenerrima
I iverwort

I uminous

moss

SCAPUND

SCHIPEN ( )

BLINACU ( )

SELICAL ( )

SELICAM ( )

SELIDON ( )

SELISUB ( )

SELITRI ( )

SPHAANG ( )

SPHABAL ( )

SPHACAP ( )

SPHACEN ( )

SPHACOM ( )

SPHACON ( )

SPHAFAL ( )

SPHAFIM ( )

SPHAFUS ( )

SPHAGIR ( )

SPHAJEN ( )

SPHALIN ( )

SPHAMAG ( )

SPHAMAJ ( )

SPHAOBT ( )

SPHARIP ( )

SPHARUS ( )

SPHASQU ( )

SPHASUB ( )

SPHATER ( )

SPHAWAR ( )

SPHAWUL ( )

SPLAAMP ( )

SPLALUT ( )

SPLARUB ( )

SPLASPH ( )

SPLAVAS ( )

TAYLACU ( )

TAYLFRO ( )

TAYLHOR ( )

TAYLLIN ( )

TAYLSER ( )

TAYLSPL ( )

TETRANG ( )

TETRMNI ( )

TETRURC ( )

VOITNIV ( )

TETRPEL ( )

CLAOBOL ( )

HAPLMIC ()

HAPLVIR ()

HELOBLA ( )

HETEDIM ( )

MYURJUL ( )

MYURSIB ( )

MYURTEN ( ) 
THUIDIACEAE

THUIDIACEAE

THUIDIACEAE

THUIDIACEAE

THUIDIACEAE

TIMMIACEAE

TIMMIACEAE

TIMMIACEAE

TIMMIACEAE

\author{
Pseudoleskeella catenulata \\ Pseudoleskeella tectorum \\ Thuidium abietinum \\ Thuidium delicatulum \\ Thuidium recognitum \\ Timmia austriaca \\ Timmia megapolitana \\ Timmia norvegica \\ Timmia sibirica
}

PSEUCAT ( )

PSEUTEC ( )

THUIABI ( )

THUIDEL ( )

THUIREC ( )

TIMMAUS ( )

TIMMMEG ( )

TIMMNOR ( )

TIMMSIB ( ) 


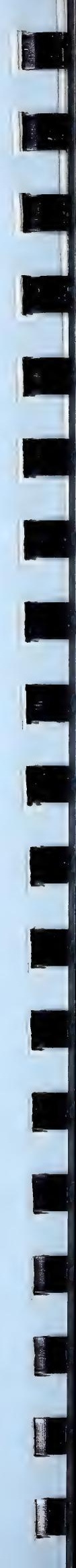


FAMILY

LATIN NAME

ENGLISH COMMON NAME

CODE

CHECK

ACAROSPORACEAE

ACAROSPORACEAE

ACAROSPORACEAE

ACAROSPORACEAE

ACAROSPORACEAE

ACAROSPORACEAE

ACAROSPORACEAE

ACAROSPORACEAE

ACAROSPORACEAE

ACAROSPORACEAE

ACAROSPORACEAE

ACAROSPORACEAE

ACAROSPORACEAE

ACAROSPORACEAE

ACAROSPORACEAE

ACAROSPORACEAE

ARTHON I ACEAE

ARTHON I ACEAE

ARTHOPYRENIACEAE

BAEOMYCETACEAE

BUELLIACEAE

BUELLIACEAE

BUELLIACEAE

BUELLIACEAE

BUELLIACEAE

BUELLIACEAE

BUELLIACEAE

BUELLIACEAE

BUELLIACEAE

BUELLIACEAE

BUELLIACEAE

BUELLIACEAE

BUELLI ACEAE

BUELLIACEAE

BUELLIACEAE

BUELLI ACEAE

BUELLIACEAE

BUELLIACEAE

BUELLIACEAE

BUELLIACEAE

BUELLIACEAE

BUELLIACEAE

BUELLIACEAE

BUELLIACEAE

CALICIACEAE

CALICIACEAE

CALICIACEAE

CALICIACEAE

CALICIACEAE

CALICIACEAE

CALICIACEAE

CANDELARIACEAE

CANDELARIACEAE

CANDELARIACEAE
Acarospora arenacea

Acarospora cervina

Acarospora chlorophana

Acarospora fuscata

Acarospora heppii

Acarospora oligospora

Acarospora schleicheri

Acarospora smaragdula

Acarospora strigata

Acarospora veronensis

Glypholecia scabra

Polysporina simplex

Sarcogyne novomexicana

Sarcogyne privigna

Sporastatia polyspora

Sporastatia testudinea

Arthonia intexta

Arthonia patellulata

Arthopyrenia punctiformis

Baeomyces rufus

Buellia disciformis

Buellia elegans

Buellia erubescens

Buellia lactoidea

Buellia papillata

Buellia punctata

Buellia spuria

Buellia triphragmioides

Buellia vilis

Dimelaena oreina

Lichenothelia scopularia

Orphniospora moriopsis

Phaeorrhiza nimbosa

Rinodina annulata

Rinodina archaea

Rinodina bischoffii

Rinodina calcigena

Rinodina exigua

Rinodina milliaria

Rinodina mniaraea

Rinodina polyspora

Rinodina pyrina

Rinodina roscida

Rinodina turfacea

Calicium abietinum

Calicium salicinum

Calicium viride

Chaenotheca chrysocephala

Chaenotheca furfuracea

Mycocalicium subtile

Phaeocalicium populneum

Candelaria concolor

Candelariella aurella

Candelariella canadensis
ACARARE ( )

ACARCER ( )

ACARCHL ( )

ACARFUS ( )

ACARHEP ( )

ACAROLI ( )

ACARSCH ( )

ACARSMA ( )

ACARSTR ( )

ACARVER ( )

GLYPSCA ( )

POLYSIM ( )

SARCNOV ( )

SARCPRI ( )

SPORPOL ( )

SPORTES ( )

ARTHINT ( )

ARTHPAT ( )

ARTHPUN ( )

BAEORUF ( )

BUELDIS ( )

BUELELE ( )

BUELERU ( )

BUELLAC ( )

BUELPAP ( )

BUELPUN ( )

BUELSPU ( )

BUELTRI ( )

BUELVIL ( )

DIMEORE ( )

LICHSCO ( )

ORPHMOR ( )

PHAENIM ( )

RINOANN ( )

RINOARC ( )

RINOBIS ( )

RINOCAL ( )

RINOEXI ( )

RINOMIL ( )

RINOMNI ( )

RINOPOL ( )

RINOPYR ( )

RINOROS ( )

RINOTUR ( )

CALIAB| ()

CALISAL ( )

CALIVIR ( )

CHAECHR ( )

CHAEFUR ( )

MYCOSUB ( )

PHAEPOP ( )

CANDCON ( )

CANDAUR ( )

CANDCAN ( ) 
CANDELARIACEAE CANDELARIACEAE CANDELARIACEAE CLADONIACEAE CLADONIACEAE CLADONIACEAE CLADON IACEAE CLADONIACEAE CLADONIACEAE CLADONIACEAE CLADONIACEAE CLADON IACEAE CLADONIACEAE CLADONIACEAE CLADON IACEAE CLADONIACEAE CLADONIACEAE CLADONIACEAE CLADONIACEAE CLADONIACEAE CLADONIACEAE CLADON IACEAE CLADONIACEAE CLADONIACEAE CLADONIACEAE CLADONIACEAE CLADON IACEAE CLADONIACEAE CLADONIACEAE CLADONIACEAE CLADON IACEAE CLADON IACEAE CLADON IACEAE CLADONIACEAE CLADONIACEAE CLADONIACEAE CLADONIACEAE CLADONIACEAE CLADON IACEAE CLADONIACEAE CLADONIACEAE CLADONIACEAE CLADON IACEAE CLADON I ACEAE CLADONIACEAE CLADON I ACEAE CLADON IACEAE CLADON IACEAE CLADONIACEAE CLADON IACEAE CLADON IACEAE CLADONIACEAE CLADONIACEAE CLADON IACEAE

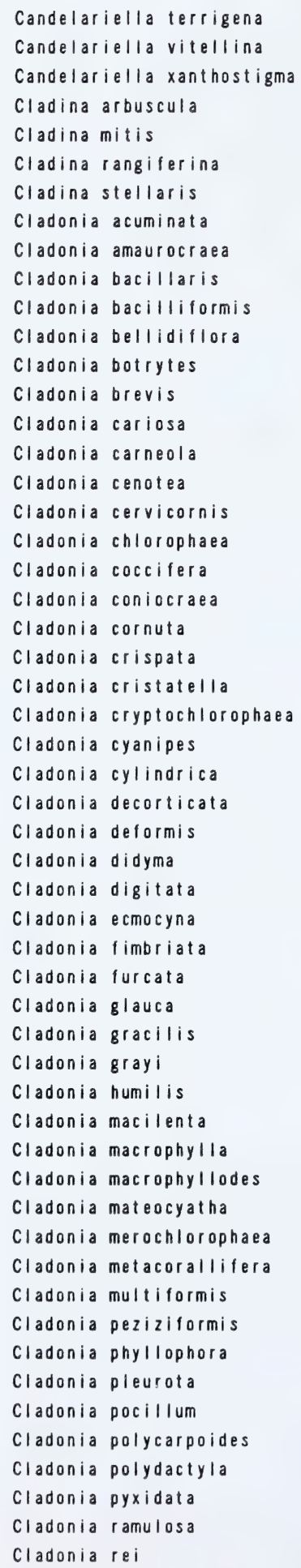

CANDTER ( )

CANDVIT ( )

CANDXAN ( )

CLADARB ( )

CLADMIT ( )

CLADRAN ( )

CLADSTE ( )

CLADACU ( )

CLADAMA ( )

CLADBAC ( )

CLADBAI ( )

CLADBEL ( )

CLADBOT ( )

CLADBRE ( )

CLADCAR ( )

CLADCAN ( )

CLADCEN ( )

CLADCER ( )

CLADCHL ( )

CLADCOC ( )

CLADCON ( )

CLADCOR ( )

CLADCRI ( )

CLADCRS ( )

CLADCRY ( )

CLADCYA ( )

CLADCYL ( )

CLADDEC ( )

CLADDEF ( )

CLADDID ( )

CLADDIG ( )

CLADECM ( )

CLADFIM ( )

CLADFUR ( )

CLADGLA ( )

CLADGRA ( )

CLADGRY ( )

CLADHUM ( )

CLADMAC ( )

CLADMAR ( )

CLADMAY ( )

CLADMAT ( )

CLADMER ( )

CLADMET ( )

CLADMUL ( )

CLADPEZ ( )

CLADPHY ( )

CLADPLE ( )

CLADPOC ( )

CLADPOY ( )

CLADPOL ( )

CLADPYX ( )

CLADRAM ( )

CLADREI ( ) 
FAMILY LATIN NAME

Cladonia robbinsii

Cladonia scabriuscula

Cladonia simulata

Cladonia sobolescens

Cladonia squamosa

Cladonia stricta

Cladonia subcervicornis

Cladonia subfurcata

Cladonia subulata

Cladonia sulphurina

Cladonia symphycarpa

Cladonia turgida

Cladonia uncialis

Spilonema revertens

Collema bachmanianum

Collema ceraniscum

Collema crispum

Collema cristatum

Collema flaccidum

Collema furfuraceum

Collema fuscovirens

Collema limosum

Collema multipartitum

Collema nigrescens

Collema polycarpon

Collema subflaccidum

Collema tenax

Collema undulatum

Lempholemma radiatum

Leptogium burnetiae

Leptogium furfuraceum

Leptogium gelatinosum

Leptogium hirsutum

Leptogium lichenoides

Leptogium saturninum

Leptogium tenuissimum

Leptogium teretiusculum

Cyphelium inquinans

Cyphelium notarisii

Cyphelium pinicola

Cyphelium tigillare

Diploschistes scruposus

Xylographa abietina

Xylographa vitiligo

Aspicilia alphoplaca

Aspicilia caesiocinerea

Aspicilia calcarea

Aspicilia candida

Aspicilia cinerea

Aspicilia elevata

Aspicilia nikrapensis

Aspicilia pergibbosa

Aspicilia supertegens

Bellemerea alpina
ENGLISH COMMON NAME

CODE CHECK

CLADROB ( )

CLADSCA ( )

CLADSIM ( )

CLADSOB ( )

CLADSQU ( )

CLADSTR ( )

CLADSUE ( )

CLADSUF ( )

CLADSUB ( )

CLADSUL ( )

CLADSYM ( )

CLADTUR ( )

CLADUNC ( )

SPILREV ( )

COLLBAC ( )

COLLCER ( )

COLLCRS ( )

COLLCRI ( )

COLLFLA ( )

COLLFUR ( )

COLLFUS ( )

COLLLIM ( )

COLLMUL ( )

COLLNIG ( )

COLLPOL ( )

COLLSUB ( )

COLLTEN ( )

COLLUND ( )

LEMPRAD ( )

LEPTBUR ( )

LEPTFUR ( )

LEPTGEL ( )

LEPTHIR ( )

LEPTLIC ( )

LEPTSAT ( )

LEPTTEN ( )

LEPTTER ( )

CYPHINQ ( )

CYPHNOT ( )

CYPHPIN ( )

CYPHTIG ()

DIPLSCR ( )

XYLOABI ( )

XYLOVIT ()

ASPIALP ( )

ASPICAE ( )

ASPICAL ( )

ASPICAN ( )

ASPICIN ( )

ASPIELE ()

ASPINIK ( )

ASPIPER ( )

ASPISUP ( )

BELLALP ( ) 
LECANORACEAE LECANORACEAE LECANORACEAE LECANORACEAE LECANORACEAE LECANORACEAE LECANORACEAE LECANORACEAE LECANORACEAE LECANORACEAE LECANORACEAE LECANORACEAE LECANORACEAE LECANORACEAE LECANORACEAE LECANORACEAE LECANORACEAE LECANORACEAE LECANORACEAE LECANORACEAE LECANORACEAE LECANORACEAE LECANORACEAE LECANORACEAE LECANORACEAE LECANORACEAE LECANORACEAE LECANORACEAE LECANORACEAE LECANORACEAE LECANORACEAE LECANORACEAE LECANORACEAE LECANORACEAE LECANORACEAE LECANORACEAE LECANORACEAE LECANORACEAE LECANORACEAE LECANORACEAE LECANORACEAE LECANORACEAE LECANORACEAE LECANORACEAE LECANORACEAE LECANORACEAE LECANORACEAE LECANORACEAE LECIDEACEAE LECIDEACEAE LECIDEACEAE LECIDEACEAE LECIDEACEAE LECIDEACEAE

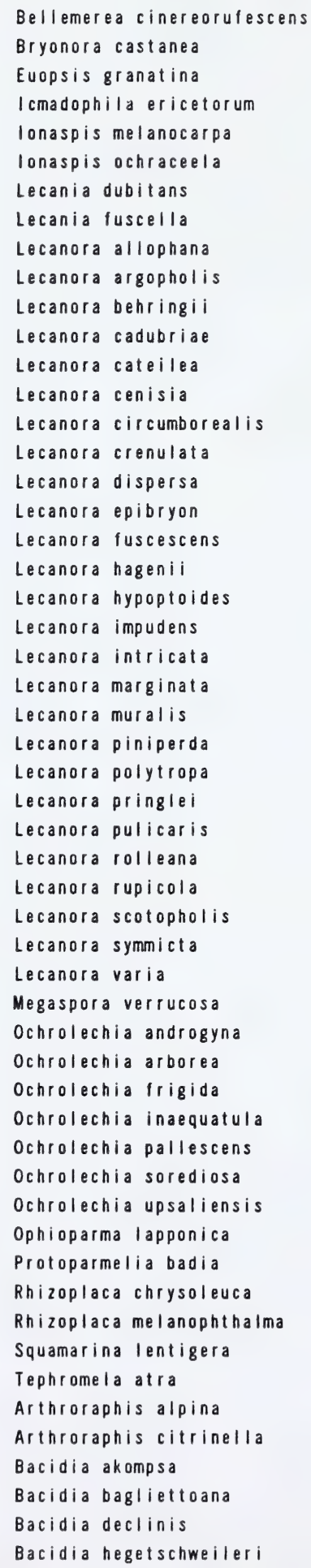

BELLCIN ( ) BRYOCAS ( ) 
LECIDEACEAE

LECIDEACEAE

LECIDEACEAE

LECIDEACEAE

LECIDEACEAE

LECIDEACEAE

LECIDEACEAE

LECIDEACEAE

LECIDEACEAE

LECIDEACEAE

LECIDEACEAE

LECIDEACEAE

LECIDEACEAE

LECIDEACEAE

LECIDEACEAE

LECIDEACEAE

LECIDEACEAE

LECIDEACEAE

LECIDEACEAE

LECIDEACEAE

LECIDEACEAE

LECIDEACEAE

LECIDEACEAE

LECIDEACEAE

LECIDEACEAE

LECIDEACEAE

LECIDEACEAE

LECIDEACEAE

LECIDEACEAE

LECIDEACEAE

LECIDEACEAE

LECIDEACEAE

LECIDEACEAE

LECIDEACEAE

LECIDEACEAE

LECIDEACEAE

LECIDEACEAE

LECIDEACEAE

LECIDEACEAE

LECIDEACEAE

LECIDEACEAE

LECIDEACEAE

LECIDEACEAE

LECIDEACEAE

LECIDEACEAE

LECIDEACEAE

LECIDEACEAE

LECIDEACEAE

LECIDEACEAE

LECIDEACEAE

LECIDEACEAE

LECIDEACEAE

LECIDEACEAE

LECIDEACEAE

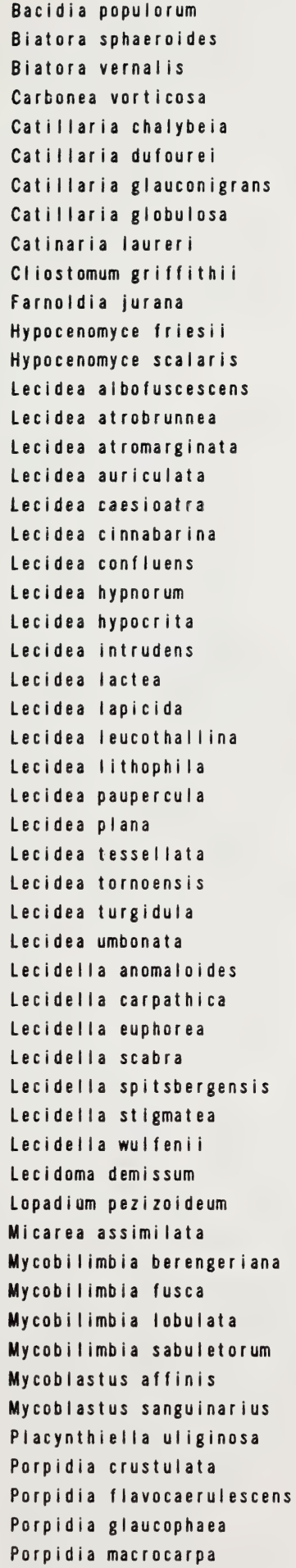

\begin{tabular}{|c|c|}
\hline POP & \\
\hline B।ATSPH & \\
\hline BIATVER & \\
\hline CARBVOR & \\
\hline CATICHA & \\
\hline CATIDUF & \\
\hline ATIGLA & \\
\hline ATIGLO & \\
\hline CATILAU & \\
\hline CLIOGRI & \\
\hline FARN JUR & \\
\hline HYPOFRI & \\
\hline HYPOSCA & \\
\hline LECIALB & \\
\hline LECIATR & \\
\hline LECIATM & \\
\hline LECIAUR & \\
\hline LECICAE & \\
\hline LECICIN & \\
\hline LECICON & \\
\hline LECIHYN & \\
\hline LECIHYP & \\
\hline LECIINT & \\
\hline LECILAC & \\
\hline LECILAP & \\
\hline LECILEU & \\
\hline LECILIT & \\
\hline LECIPAU & \\
\hline LECIPLA & \\
\hline LECITES & \\
\hline LECITOR & \\
\hline LECITUR & \\
\hline LECIUMB & \\
\hline LECIANO & \\
\hline LECICAR & \\
\hline LECIEUP & \\
\hline LECISCA & \\
\hline LECISPI & \\
\hline LECISTI & \\
\hline LECIWUL & \\
\hline LECIDEM & \\
\hline LOPAPEZ & \\
\hline MICAASS & \\
\hline MYCOBER & \\
\hline MYCOOBS & \\
\hline MYCOLOB & \\
\hline MYCOSAB & \\
\hline MYCOAFF & \\
\hline MYCOSAN & \\
\hline PLACULI & \\
\hline PORPCRU & \\
\hline ORPFLA & \\
\hline ORPGLA & \\
\hline & \\
\hline
\end{tabular}


LECIDEACEAE

LECIDEACEAE

LECIDEACEAE

LECIDEACEAE

LECIDEACEAE

LECIDEACEAE

LECIDEACEAE

LECIDEACEAE

LECIDEACEAE

LECIDEACEAE

LECIDEACEAE

LECIDEACEAE

LECIDEACEAE

LECIDEACEAE

LECIDEACEAE

LECIDEACEAE

LECIDEACEAE

LECIDEACEAE

LECIDEACEAE

LECIDEACEAE

LECIDEACEAE

LECIDEACEAE

LECIDEACEAE

LECIDEACEAE

LECIDEACEAE

LECIDEACEAE

LECIDEACEAE

LECIDEACEAE

LECIDEACEAE

LEPRAR I ACEAE

LEPRAR IACEAE

LEPRAR I ACEAE

LEPRARIACEAE

LI CHINACEAE

LICHINACEAE

IICHINACEAE

LICHINACEAE

NEPHROMATACEAE

NEPHROMATACEAE

NEPHROMATACEAE

NEPHROMATACEAE

NEPHROMATACEAE

NEPHROMATACEAE

OPEGRAPHACEAE

PANNARIACEAE

PANNARIACEAE

PANNAR I ACEAE

PARMELIACEAE

PARMELIACEAE

PARMELIACEAE

PARMELI ACEAE

PARMELIACEAE

PARMELIACEAE

PARMELIACEAE

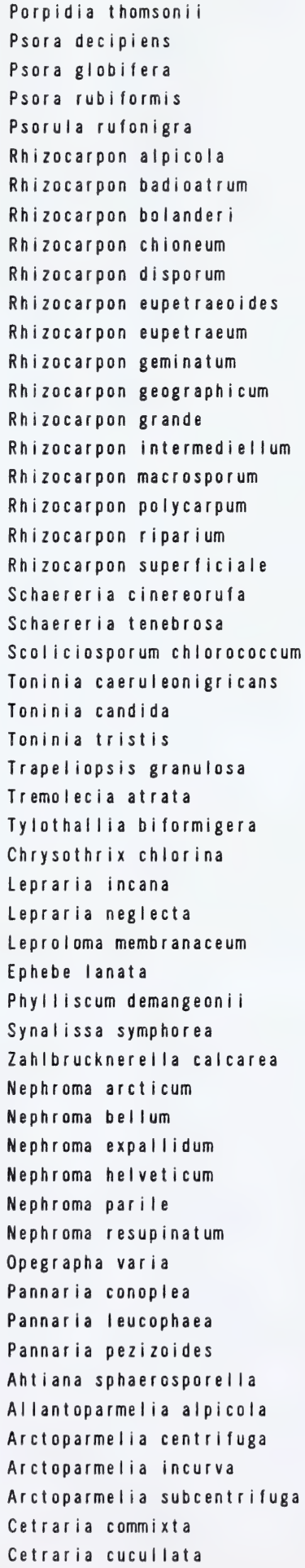

PORPTHO

PSORDEC

PSORGLO

PSORRUB

PSORRUF

RHIZALP

RHI ZBAD

RHIZBOL

RHIZCHI

RHIZDIS

RHIZEUP

RHIZEUM

RHIZGER

RHIZGEO

RHIZGRN

RHIZINT

RHI ZMAC

RHIZPOL

RHIZRIP

RHIZSUP

SCHACIN

SCHATEN

SCOLCHL

TONICAE

TONICAN

TONITRI

TRAPGRA

TREMATR

TYLOBIF

CHRYCHL

LEPRINC

LEPRNEG

LEPRMEM

EPHELAN

PHYLDEM

SYNASYM

ZAHLCAL

NEPHARC

NEPHBEL

NEPHEXP

NEPHHEL

NEPHPAR

NEPHRES

OPEGVAR

PANNCON

PANNLEU

PANNPEZ

AHTISPH

ALLAALP

ARCTCEN

ARCTINC

ARCTSUB

CETRCOM

CETRCUC
( ) 
PARMELIACEAE PARMELIACEAE PARMELIACEAE PARMELIACEAE PARMELIACEAE PARMELIACEAE PARMEL IACEAE PARMELIACEAE PARMEL IACEAE PARMEL IACEAE PARMEL IACEAE PARMEL I ACEAE PARMELIACEAE PARMEL IACEAE PARMEL IACEAE PARMELIACEAE PARMEL IACEAE PARMEL IACEAE PARMELIACEAE PARMEL IACEAE PARMELIACEAE PARMELIACEAE PARMELIACEAE PARMELI ACEAE PARMELIACEAE PARMEL IACEAE PARMELIACEAE PARMELIACEAE PARMELIACEAE PARMELIACEAE PARMELIACEAE PARMELIACEAE PARMELIACEAE PARMELIACEAE PARMELIACEAE PARMELIACEAE PARMELIACEAE PARMELIACEAE PARMELIACEAE PARMELIACEAE PARMELIACEAE PARMELIACEAE PARMELIACEAE PARMELIACEAE PARMELIACEAE PARMELIACEAE PARMELIACEAE PARMELIACEAE PARMELIACEAE PARMELIACEAE PARMELIACEAE PARMELIACEAE PARMEL IACEAE PARMELIACEAE

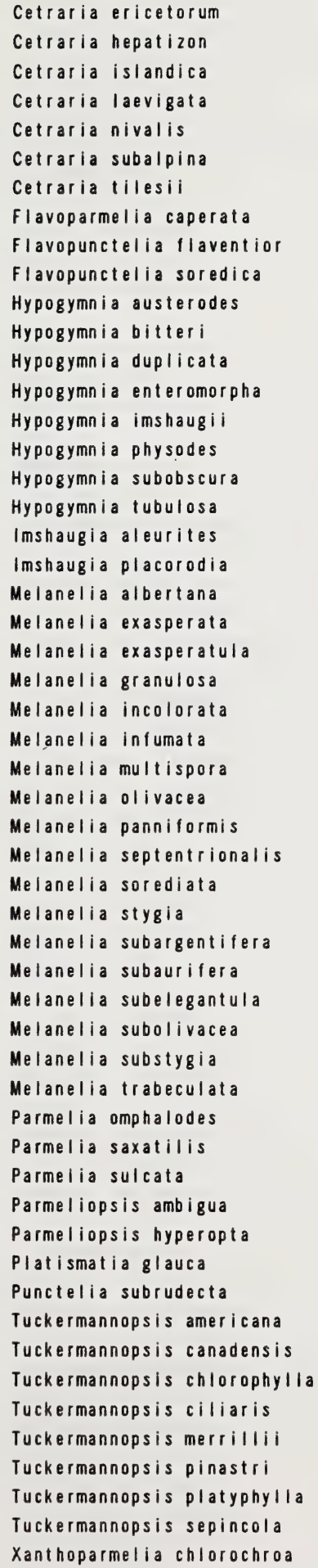

\begin{tabular}{|c|c|}
\hline & \\
\hline TRH & \\
\hline TRISL & \\
\hline ETRLAE & \\
\hline ETRNIV & \\
\hline ETRSUB & \\
\hline ETRTIL & \\
\hline LAVCAP & \\
\hline LAVFLA & \\
\hline LAVSOR & \\
\hline IYPOAUS & \\
\hline АYРОВ IT & \\
\hline AYPODUP & \\
\hline HYPOENT & \\
\hline HYPOIMS & \\
\hline HYPOPHY & \\
\hline HYPOSUB & \\
\hline HYPOTUB & \\
\hline IMSHALE & \\
\hline IMSHPLA & \\
\hline MELAALB & \\
\hline MELAEXA & \\
\hline MELAEXS & \\
\hline MELADIS & \\
\hline MELAINC & \\
\hline MELAINF & \\
\hline MELAMUL & \\
\hline MELAOLI & \\
\hline MELAPAN & \\
\hline MELASEP & \\
\hline MELASOR & \\
\hline MELASTY & \\
\hline MELASUA & \\
\hline MELASUB & \\
\hline MELASUE & \\
\hline MELASUO & \\
\hline MELASUS & \\
\hline MELATRA & \\
\hline PARMOMP & \\
\hline PARMSAX & \\
\hline PARMSUL & \\
\hline PARMAMB & \\
\hline PARMHYP & \\
\hline PLATGLA & \\
\hline PUNCSUR & \\
\hline TUCKAME & \\
\hline TUCKCAN & \\
\hline TUCKCHL & \\
\hline TUCKCIL & \\
\hline TUCKMER & \\
\hline TUCKPIN & \\
\hline TUCKPLA & \\
\hline TUCKSEP & \\
\hline ANTCH & \\
\hline
\end{tabular}


PARMELIACEAE

PARMELIACEAE

PARMELIACEAE

PARMEL IACEAE

PELTIGERACEAE

PELTIGERACEAE

PELTIGERACEAE

PELTIGERACEAE

PELTIGERACEAE

PELTIGERACEAE

PELTIGERACEAE

PELTIGERACEAE

PELTIGERACEAE

PELTIGERACEAE

PELTIGERACEAE

PELTIGERACEAE

PELTIGERACEAE

PELTIGERACEAE

PELTIGERACEAE

PELTIGERACEAE

PELTIGERACEAE

PELTIGERACEAE

PELTIGERACEAE

PELTIGERACEAE

PELTIGERACEAE

PERTUSARIACEAE

PERTUSARIACEAE

PERTUSARIACEAE

PERTUSARIACEAE

PERTUSARIACEAE

PERTUSARIACEAE

PERTUSARIACEAE

PERTUSARIACEAE

PHYSCIACEAE

PHYSCIACEAE

PHYSCIACEAE

PHYSCIACEAE

PHYSCIACEAE

PHYSCIACEAE

PHYSCIACEAE

PHYSCIACEAE

PHYSCIACEAE

PHYSCIACEAE

PHYSCIACEAE

PHYSCIACEAE

PHYSCIACEAE

PHYSCIACEAE

PHYSCIACEAE

PHYSCIACEAE

PHYSCIACEAE

PHYSCIACEAE

PHYSCIACEAE

PHYSCIACEAE

PHYSCIACEAE

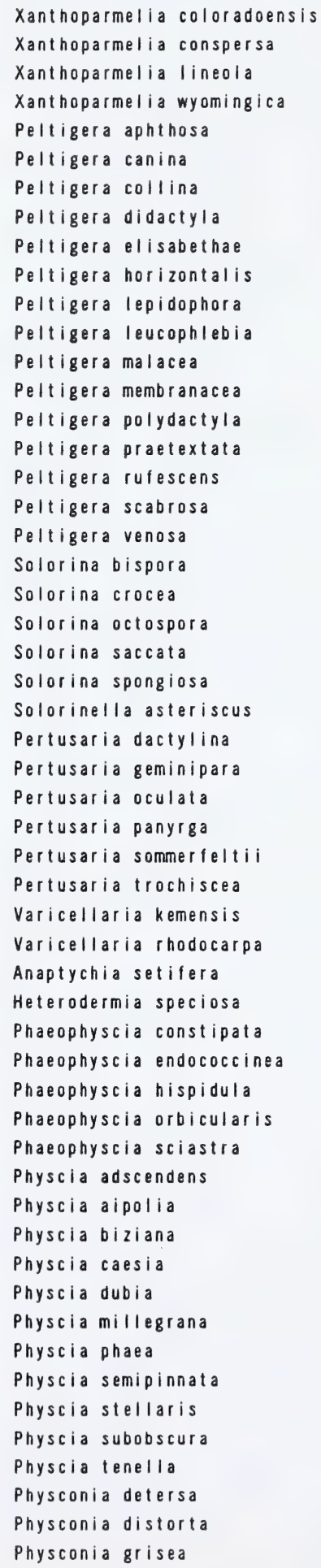

XANTTAR ( )

XANTCON ( )

XANTLIN ( )

XANTWYO ( )

PELTAPH ( )

PELTCAN ( )

PELTCOL ( )

PELTDID ( )

PELTELI ( )

PELTHOR ( )

PELTLEP ( )

PELTLEU ( )

PELTMAL ( )

PELTMEM ( )

PELTPOL ( )

PELTPRA ( )

PELTRUF ( )

PELTSCA ( )

PELTVEN ( )

SOLOBIS ( )

SOLOCRO ( )

SOLOOCT ( )

SOLOSAC ( )

SOLOSPO ( )

SOLOAST ( )

PERTDAC ( )

PERTGEM ( )

PERTOCU ( )

PERTPAN ( )

PERTSOM ( )

PERTTRO ( )

VARIKEM ( )

VARIRHO ( )

ANAPSET ( )

HETESPE ( )

PHAECON ( )

PHAEEND ( )

PHAEHIS ( )

PHAEORB ( )

PHAESCI ( )

PHYSADS ( )

PHYSAIP ( )

PHYSBIZ ( )

PHYSCAE ( )

PHYSOUB ( )

PHYSMIL ( )

PHYSPHA ( )

PHYSSEM ( )

PHYSSTE ( )

PHYSSUB ( )

PHYSTEN ( )

PHYSDET ( )

PHYSDIS ( )

PHYSGRI ( ) 
FAMILY LATIN NAME

ENGLISH COMMON NAME

CODE

CHECK

PHYSCIACEAE

PHYSCIACEAE

PLACYNTHIACEAE

PLACYNTHIACEAE

PLACYNTHIACEAE

PLACYNTHIACEAE

PLACYNTHIACEAE

PYRENULACEAE

RAMALI NACEAE

RAMALINACEAE

RAMALINACEAE

RAMALINACEAE

RAMALINACEAE

RAMALINACEAE

RAMALINACEAE

RAMALINACEAE

RAMALINACEAE

RAMALINACEAE

SPHAEROPHORACEAE

STEREOCAULACEAE

STEREOCAULACEAE

STEREOCAULACEAE

STEREOCAULACEAE

STEREOCAULACEAE

STEREOCAULACEAE

STEREOCAULACEAE

STEREOCAULACEAE

STEREOCAULACEAE

STEREOCAULACEAE

STICTACEAE

STICTACEAE

STICTACEAE

TELOSCHISTACEAE

TELOSCHISTACEAE

TELOSCHISTACEAE

TELOSCHISTACEAE

TELOSCHISTACEAE

TELOSCH I STACEAE

TELOSCHISTACEAE

TELOSCHISTACEAE

TELOSCHISTACEAE

TELOSCHISTACEAE

TELOSCHISTACEAE

TELOSCHISTACEAE

TELOSCHISTACEAE

TELOSCHISTACEAE

TELOSCHISTACEAE

TELOSCHISTACEAE

TELOSCHISTACEAE

TELOSCHISTACEAE

TELOSCHISTACEAE

TELOSCHISTACEAE

TELOSCHISTACEAE

TELOSCHISTACEAE
Physconia muscigena

Physconia perisidiosa

Placynthiun asperellum

Placynthium nigrum

PIacynthium stenophy I I um

PIacynthium subradiatum

Psoroma hypnorum

Pyrenula leucoplaca

Ramalina americana

Ramalina calicaris

Ramalina dilacerata

Ramalina farinacea

Ramalina intermedia

Ramalina obtusata

Ramalina pollinaria

Ramalina roesleri

Ramalina sinensis

Ramalina thrausta

Sphaerophorus globosus

Leprocaulon subalbicans

Stereocaulon alpinum

Stereocaulon botryosum

Stereocaulon condensatum

Stereocaulon dactylophyl I um

Stereocaulon paschale

Stereocaulon pileatum

Stereocaulon rivulorum

Stereocaulon saxatile

Stereocaulon tomentosum

Lobaria linita

Lobaria oregana

Lobaria pulmonaria

Caloplaca atroalba

Caloplaca cerina

Caloplaca citrina

Caloplaca cladodes

Caloplaca epithallina

Caloplaca ferruginea

Caloplaca flavorubescens

Caloplaca flavovirescens

Caloplaca fraudans

Caloplaca holocarpa

Caloplaca jungermanniae

Caloplaca lactea

Caloplaca saxicola

Caloplaca sinapisperma

Caloplaca tetraspora

Caloplaca trachyphylla

Caloplaca ulmorum

Caloplaca vitellinula

Fulgensia bracteata

Fulgensia fulgens

Protoblastenia rupestris

Xanthoria candelaria
PHYSMUS ( )

PHYSPER ( )

PLACASP ( )

PLACNIG ( )

PLACSTE ( )

PLACSUB ( )

PSORHYP ( )

PYRELEU ( )

RAMAAME ( )

RAMACAL ( )

RAMADIL ( )

RAMAFAR ( )

RAMAINT ( )

RAMAOBT ( )

RAMAPOL ( )

RAMAROE ( )

RAMASIN ( )

RAMATHR ( )

SPHAGLO ( )

LEPRSUB ( )

STERALP ( )

STERBOT ( )

STERCON ( )

STERDAC ( )

STERPAS ( )

STERPIL ( )

STERRIV ( )

STERSAX ( )

STERTOM ( )

LOBALIN ( )

LOBAORE ( )

LOBAPUL ( )

CALOATR ( )

CALOCER ( )

CALOCIT ( )

CALOCLA ( )

CALOEPI ( )

CALOFER ( )

CALOFLR ( )

CALOFLA ( )

CALOFRA ( )

CALOHOL ( )

CALOJUN ( )

CALOLAC ( )

CALOSAX ( )

CALOSIN ( )

CALOTET ( )

CALOTRA ( )

CALOULM ( )

CALOVIT ( )

FULGBRA ( )

FULGFUL ( )

PROTRUP ( )

XANTCAN ( ) 
TELOSCHISTACEAE TELOSCHISTACEAE TELOSCHISTACEAE TELOSCHISTACEAE UMBILICARIACEAE UMBILICARIACEAE UMB ILICARIACEAE UMB ILICAR I ACEAE UMB I LI CARIACEAE UMB I LI CAR I ACEAE UMBILICARIACEAE UMB ILICAR IACEAE UMB I LICARIACEAE UMB I LICARIACEAE UMBILICARIACEAE UMB ILICARIACEAE UMBILICARIACEAE UMBILICARIACEAE UMB ILICARIACEAE UMBILICARIACEAE UMBILICARIACEAE UMBILICARIACEAE USNEACEAE USNEACEAE USNEACEAE USNEACEAE USNEACEAE USNEACEAE USNEACEAE USNEACEAE USNEACEAE USNEACEAE USNEACEAE USNEACEAE USNEACEAE USNEACEAE USNEACEAE USNEACEAE USNEACEAE USNEACEAE USNEACEAE USNEACEAE USNEACEAE USNEACEAE USNEACEAE USNEACEAE USNEACEAE USNEACEAE USNEACEAE USNEACEAE USNEACEAE USNEACEAE USNEACEAE USNEACEAE

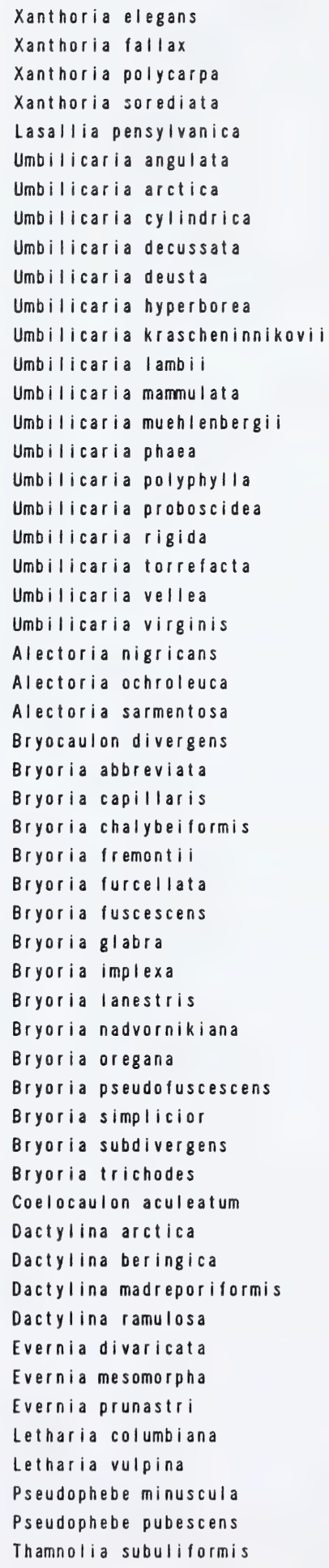

old man's beard old man's beard old man's beard old man's beard old man's beard old man's beard old man's beard old man's beard old man's beard old man's beard old man's beard old man's beard old man's beard old man's beard old man's beard

\begin{tabular}{|c|c|}
\hline ANTELEE & ( \\
\hline XANTFAL & $(1)$ \\
\hline XANTPOL & ( \\
\hline XANTSOR & ( \\
\hline LASAPEN & ( \\
\hline UMB IANG & ( \\
\hline UMB |ARC & ( \\
\hline UMBICYL & ( \\
\hline UMBIDEC & ( ) \\
\hline UMBIDEU & ( ) \\
\hline UMBIHYP & ( ) \\
\hline UMB IKRA & ( \\
\hline UMB I LAM & ( \\
\hline UMB IMAM & ( \\
\hline UMB IMUE & ( \\
\hline UMBIPHA & ( \\
\hline UMBIPOL & ( \\
\hline UMBIPRO & ( \\
\hline UMBIRIG & ( ) \\
\hline UMBITOR & () \\
\hline UMB I VEL & ( ) \\
\hline UMBIVIR & ( ) \\
\hline ALECN IG & ( ) \\
\hline ALECOCH & ( ) \\
\hline ALECSAR & ( ) \\
\hline BRYODIV & ( ) \\
\hline$B R Y O A B B$ & ( ) \\
\hline BRYOCAP & ( \\
\hline BRYOCHA & ( ) \\
\hline BRYOFRE & ( ) \\
\hline BRYOFUR & ( ) \\
\hline BRYOFUS & ( ) \\
\hline BRYOGLA & ( ) \\
\hline BRYOIMP & ( ) \\
\hline BRYOLAN & ( ) \\
\hline BRYONAD & ( ) \\
\hline BRYOORE & ( ) \\
\hline BRYOPSE & ( ) \\
\hline BRYOSIM & ( ) \\
\hline BRYOSUB & ( ) \\
\hline BRYOTRI & ( ) \\
\hline COELACU & ( ) \\
\hline DACTARC & ( ) \\
\hline DACTBER & ( ) \\
\hline DACTMAD & ( ) \\
\hline DACTRAM & ( ) \\
\hline EVERDIV & ( ) \\
\hline EVERMES & ( ) \\
\hline EVERPRU & ( ) \\
\hline LETHCOL & ( ) \\
\hline LETHVUL & ( ) \\
\hline PSEUM IN & ( \\
\hline PSEUPUB & \\
\hline THAMSUB & \\
\hline
\end{tabular}


USNEACEAE

USNEACEAE

USNEACEAE

USNEACEAE

USNEACEAE

USNEACEAE

USNEACEAE

USNEACEAE

USNEACEAE

USNEACEAE

USNEACEAE

USNEACEAE

USNEACEAE

VERRUCARIACEAE

VERRUCARIACEAE

VERRUCAR IACEAE

VERRUCARIACEAE

VERRUCAR IACEAE

VERRUCAR IACEAE

VERRUCAR IACEAE

VERRUCAR IACEAE

VERRUCAR IACEAE

VERRUCAR IACEAE

VERRUCAR IACEAE

VERRUCAR IACEAE

VERRUCAR IACEAE

VERRUCARIACEAE

VERRUCAR IACEAE

VERRUCARIACEAE

VERRUCAR IACEAE

VERRUCARIACEAE

VERRUCAR IACEAE

VERRUCAR IACEAE

VERRUCARIACEAE

VERRUCAR IACEAE

VERRUCAR IACEAE

VERRUCARIACEAE

VERRUCAR IACEAE

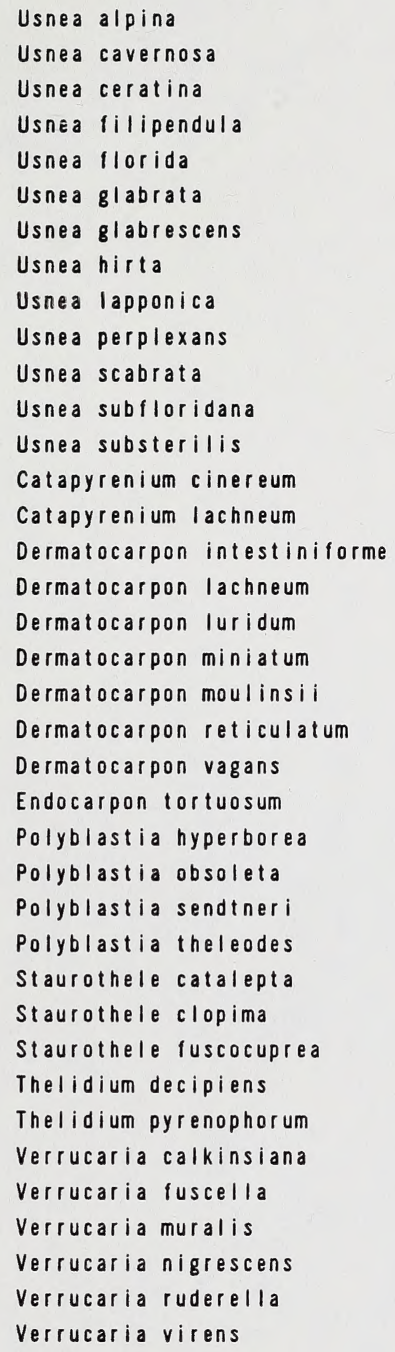

\begin{tabular}{|c|c|}
\hline SONEA & \\
\hline SNECAV & \\
\hline IECER & \\
\hline$F I L$ & \\
\hline EFLO & \\
\hline NEGLT & \\
\hline SNEGLA & \\
\hline SNEHIR & \\
\hline NELAP & \\
\hline NEPER & \\
\hline NESCA & \\
\hline NESUB & \\
\hline NESUS & \\
\hline ATACIN & \\
\hline TALAC & \\
\hline EMINT & \\
\hline RMLAC & \\
\hline ERMLUR & \\
\hline RMMIN & \\
\hline RMMOU & \\
\hline RMRET & \\
\hline RMVAG & \\
\hline IDOTOR & \\
\hline LLYHYP & \\
\hline OLYOBS & \\
\hline OLYSEN & \\
\hline OLYTHE & \\
\hline TAUCAT & \\
\hline TAUCLO & \\
\hline STAUFUS & \\
\hline HELDEC & \\
\hline HELPYR & \\
\hline IERRCAL & \\
\hline VERRFUS & \\
\hline VERRMUR & \\
\hline VERRNIG & \\
\hline ERRRUD & \\
\hline RRVIR & \\
\hline
\end{tabular}




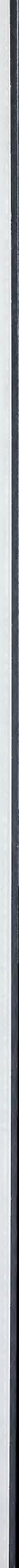



